\title{
CONTROLLING SECONDARY FLOWS IN VERY HIGHLY-LOADED LOW-PRESSURE TURBINE CASCADES
}

by

\section{Daniel C. Knezevici}

\begin{abstract}
A thesis submitted to the Faculty of Graduate Studies and Postdoctoral Affairs in partial fulfillment of the requirements for the degree of
\end{abstract}

\section{Doctor of Philosophy}

in Aerospace Engineering

Ottawa-Carleton Institute for Mechanical \& Aerospace Engineering

Department of Mechanical \& Aerospace Engineering

Carleton University

Ottawa, Ontario, Canada

(C)Daniel C. Knezevici

September 2011 
Library and Archives

Canada

Published Heritage

Branch

395 Wellington Street

Ottawa ON K1A ON4

Canada
Bibliothèque et

Archives Canada

Direction du

Patrimoine de l'édition

395 , rue Wellington

Ottawa ON K1A ON4

Canada
Your file Votre référence

ISBN: 978-0-494-89339-5

Our file Notre référence

ISBN: 978-0-494-89339-5

\section{NOTICE:}

The author has granted a nonexclusive license allowing Library and Archives Canada to reproduce, publish, archive, preserve, conserve, communicate to the public by telecommunication or on the Internet, loan, distrbute and sell theses worldwide, for commercial or noncommercial purposes, in microform, paper, electronic and/or any other formats.

The author retains copyright ownership and moral rights in this thesis. Neither the thesis nor substantial extracts from it may be printed or otherwise reproduced without the author's permission.
AVIS:

L'auteur a accordé une licence non exclusive permettant à la Bibliothèque et Archives Canada de reproduire, publier, archiver, sauvegarder, conserver, transmettre au public par télécommunication ou par l'Internet, prêter, distribuer et vendre des thèses partout dans le monde, à des fins commerciales ou autres, sur support microforme, papier, électronique et/ou autres formats.

L'auteur conserve la propriété du droit d'auteur et des droits moraux qui protege cette thèse. $\mathrm{Ni}$ la thèse ni des extraits substantiels de celle-ci ne doivent être imprimés ou autrement reproduits sans son autorisation.
In compliance with the Canadian Privacy Act some supporting forms may have been removed from this thesis.

While these forms may be included in the document page count, their removal does not represent any loss of content from the thesis.
Conformément à la loi canadienne sur la protection de la vie privée, quelques formulaires secondaires ont été enlevés de cette thèse.

Bien que ces formulaires aient inclus dans la pagination, il n'y aura aucun contenu manquant. 
Dedicated to those

who

believed. 


\section{Abstract}

This thesis documents experimental and computational results of a research program investigating the aerodynamic losses generated by extremely highly loaded low-pressure turbine blades, with particular focus on the three-dimensional flow near the endwall. The study identifies the physical mechanisms associated with loss generation and documents changes in the flow field that result from the application of passive flow control techniques. The experimental study was conducted in Carleton University's low-speed, linear cascade wind tunnel. Quantitative results include seven-hole pneumatic probe pressure measurements downstream of the cascade to assess blade row losses, as well as detailed measurements within the blade passage to track the development of flow structures. Qualitative results in the form of oil surface flow visualization on the endwall and blade suction surface are used to assist in the interpretation of the physics. The complementary computational studies were performed using Reynolds-averaged Navier-Stokes (RANS) simulations, providing detailed resolution of the various vortical structures comprising the endwall flow.

The work examined two passive flow control techniques for mitigating endwall loss. The techniques were applied to very high-lift, low-pressure turbine airfoils with the goal of expanding the design space. The airfoils and both flow control techniques were designed by Pratt \& Whitney Aircraft (PWA) using proprietary tools. It was found that endwall loss could be significantly reduced and the mechanisms of loss reduction were identified. The loss measurements have allowed plausible limits to be defined for a high-lift replacement of the baseline airfoil used in the study. 


\section{Acknowledgements}

"It was the best of times, it was the worst of times..." -Charles J. H. Dickens

No twelve words could better summarize the time spent completing this degree. These years have granted me the luxury of unrestricted exploration and the freedom to think. But the time spent in the lab, or preparing this text was not without its dark days. These frustrating times could not have been overcome without the assistance of a number of individuals.

My profound gratitude goes to my thesis supervisor, Professor Steen Sjolander. His expertise in turbomachinery aerodynamics and experimental methods were instrumental to the success of this work and to the interpretation of the data. I also thank him for the effort he put into the review of this text.

I gratefully acknowledge the financial support of Pratt \& Whitney Aircraft (PWA) as well as their permission to publish the findings presented herein. My sincerest thanks goes to Dr. Thomas Praisner who was the technical lead and primary contact at PWA throughout this work. His efforts were backed by those of Eric Grover and Eunice Allen-Bradley who designed the research airfoils and endwall contouring, respectively.

Thank you to the staff of the Department of Mechanical \& Aerospace Engineering who consistently went above and beyond the call of duty. Most notably I'd like to thank Nancy 
Powell; she must have saved me thousands of dollars over the years by reminding me to register and pay my tuition fees on time.

A number of Carleton students (past and present) had influence over this work. They paved the way by developing hardware, test procedures, supplying data and contributing to countless discussions. Thank you to Dr. Michael Benner, Dr. Ali Mahallati, Dr. Metin Yaras, Dr. Xue Feng Zhang, Tanya Ellison, Farzad Taremi, Gordon MacIsaac, Tanya Zorić and Dr. Ivan Popović.

To the employees of my favorite coffee shops and bars, thank you for supplying the fuel for my breaks.

Finally to my Parents, Maria and Lazar, and Sister, Diana, I could not have made it this far without your endless patience, love and support. Thank you. 


\section{Table of Contents}

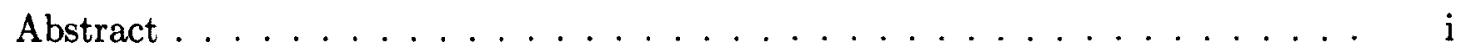

Acknowledgements $\ldots \ldots \ldots \ldots \ldots \ldots \ldots \ldots \ldots \ldots \ldots \ldots \ldots$ ii

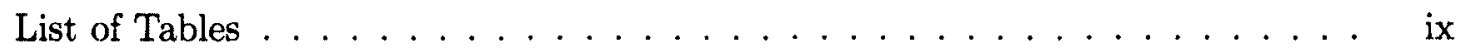

List of Figures $\ldots \ldots \ldots \ldots \ldots \ldots \ldots \ldots \ldots \ldots \ldots \ldots \ldots \ldots \ldots$

List of Symbols . . . . . . . . . . . . . . . . . . xviii

1 Introduction 1

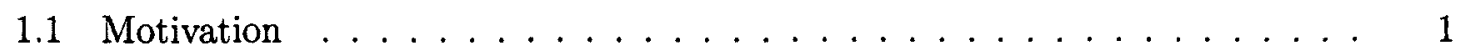

1.2 Approach $\ldots \ldots \ldots \ldots \ldots \ldots \ldots \ldots \ldots \ldots \ldots \ldots \ldots \ldots \ldots$

1.3 Thesis Objectives . . . . . . . . . . . . . . . 7

1.4 Overview of Thesis $\ldots \ldots \ldots \ldots \ldots \ldots \ldots$

2 Review of Previous Work 11

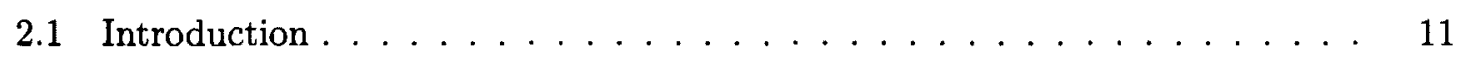

2.2 Losses in Axial Turbomachinery $\ldots \ldots \ldots \ldots \ldots \ldots$

2.3 Flow Physics in Axial Flow Turbines . . . . . . . . . . . . . . 15

2.3.1 Blade Surface Boundary Layers . . . . . . . . . . . . . 17

2.3.2 Secondary Flow and Endwall Boundary Layers . . . . . . . . 21

2.4 Blade Loading $\ldots \ldots \ldots \ldots \ldots \ldots \ldots$

2.5 Reynolds Number Effects on Loss Generation $\ldots \ldots \ldots \ldots \ldots \ldots .27$

2.6 Effect of Free Stream Turbulence Intensity on Loss Generation . . . . . . 30

2.7 Incidence Effects $\ldots \ldots \ldots \ldots \ldots \ldots \ldots \ldots \ldots \ldots \ldots \ldots$ 
2.8 Effect of Loading Distribution on Loss Generation . . . . . . . . . . . 35

2.9 Controlling Losses Generated by Secondary Flows . . . . . . . . . . . 36

2.9 .1 Endwall Fences . . . . . . . . . . . . . . . . . 36

2.9.2 Leading Edge Modifications . . . . . . . . . . . . . . . . 37

2.9.3 Axisymmetric Endwall Contouring . . . . . . . . . . . . . . . . 38

2.9.4 Non-Axisymmetric Endwall Contouring . . . . . . . . . . . . 39

2.9.4.1 Design Methodology . . . . . . . . . . . . . . . 40

2.9.4.2 Experimental Validations . . . . . . . . . . . 43

2.9.4.3 Other Applications . . . . . . . . . . . 45

2.9.5 Pressure Surface Modification . . . . . . . . . . . . . . . . . 46

3 Experimental Methods and Data Reduction Procedures 48

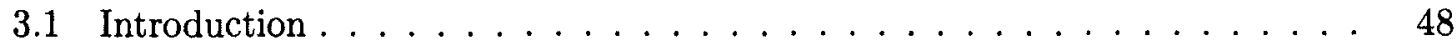

3.2 Low-Speed Wind Tunnel . . . . . . . . . . . . . . . . . . . . . . 48

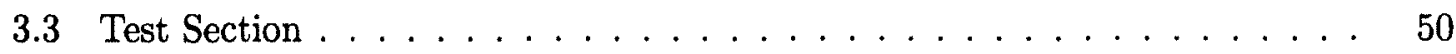

3.4 Research Cascades . . . . . . . . . . . . . . . . . . . . 51

3.5 Cascade Testing for Determination of Entropy Production . . . . . . . 56

3.6 Experimental Instrumentation . . . . . . . . . . . . . . . . 58

3.6 .1 Data Acquisition . . . . . . . . . . . . . . . . 59

3.6 .2 Pressure Transducers . . . . . . . . . . . . . . . . . 59

3.6.3 Temperature Monitor . . . . . . . . . . . . . . 60

3.6 .4 Digital Barometer . . . . . . . . . . . . . . 60

3.6.5 Stepper Motor Controller . . . . . . . . . . . . . . . . 61

3.7 Probe Calibration Apparatus $\ldots \ldots \ldots \ldots \ldots$

3.8 Experimental Uncertainty . . . . . . . . . . . . . . . . . 63

3.9 Pressure Probes . . . . . . . . . . . . . . . . . . . . . 64

3.9 .1 Pitot Tube .......................... 64

3.9.2 Three-Hole Probe . . . . . . . . . . . . . . . 65

3.9.3 Seven-Hole Probe . . . . . . . . . . . . . . . . . 66

3.10 Cascade Testing Procedures . . . . . . . . . . . . . . . . 67 
3.10.1 Wind Tunnel Calibration and Operating Point . . . . . . . . . 67

3.10 .2 Data Reduction . . . . . . . . . . . . . . . 70

3.10.2.1 Measured and Mixed-Out Losses . . . . . . . . . . . . . 70

3.10.2.2 Streamwise Vorticity, Helicity and Secondary Kinetic Energy 73

3.10 .3 Inlet and Outlet Flow Quality . . . . . . . . . . . . 76

3.10 .4 Seven-Hole Probe Axial Traverses . . . . . . . . . . . . . 79

3.10 .5 Oil Surface Flow Visualization $\ldots \ldots \ldots 8$

4 Description of Computational Method $\quad 82$

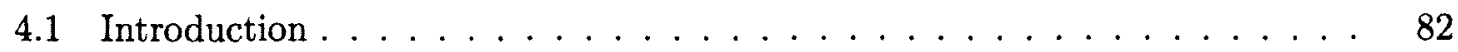

4.2 Numerical Setup . . . . . . . . . . . . . . . . 82

4.3 Grid Independence Study $\ldots \ldots \ldots \ldots$. . . . . . . . . . . . 84

4.3 .1 Two-Dimensional Domain . . . . . . . . . . . . . . . 84

4.3.2 Two-Dimensional Simulation and Boundary Conditions . . . . . 85

4.3.3 Two-Dimensional Grid Generation . . . . . . . . . . . . . 86

4.3.4 Two-Dimensional Study Results ... . . . . . . . . . . 88

4.3.5 Three-Dimensional Domain ................. 95

4.3.6 Three-Dimensional Simulation and Boundary Conditions . . . . . 95

4.3.7 Three-Dimensional Grid Generation . . . . . . . . . . . . . . 96

4.3.8 Three-dimensional Study Results . . . . . . . . . . . . . . . 97

5 Secondary Flow in a Turbine Cascade with the Implementation of NonAxisymmetric Endwall Contouring 106

5.1 Introduction . . . . . . . . . . . . . 106

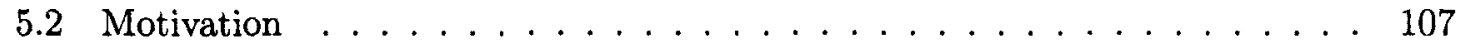

5.3 Approach . . . . . . . . . . . . . . . . 108

5.4 Endwall Contouring . . . . . . . . . . . . . . . 108

5.5 Experimental Results . . . . . . . . . . . . . . . . . . . . 109

5.5 .1 Cascade Inlet Conditions . . . . . . . . . . . . . . . . 109

5.5 .2 Blade Loading Distribution . . . . . . . . . . . . . . . . 110

5.5 .3 Flow Visualization Results . . . . . . . . . . . . . . 112 
5.5 .4 Pressure Probe Results $\ldots \ldots \ldots \ldots \ldots$

5.6 Computational Results . . . . . . . . . . . . . . . . . . . . 128

5.6.1 Blade Loading Distributions . . . . . . . . . . . . . . . . 128

5.6 .2 Flow Visualization . . . . . . . . . . . . . . . 130

5.6 .3 Predicted Losses and Flow Physics . . . . . . . . . . . . . . 133

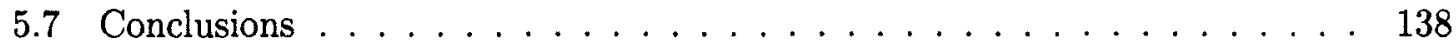

6 Secondary Flow in a High-Lift, Front-Loaded Turbine Cascade with the Implementation of Non-Axisymmetric Endwall Contouring 140

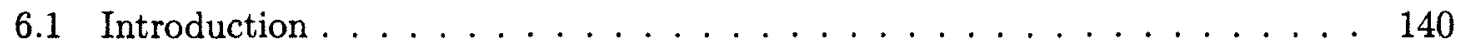

6.2 Motivation . . . . . . . . . . . . . . . . . 141

6.3 Approach . . . . . . . . . . . . . . . . . . . . . . 144

6.4 Endwall Contouring . . . . . . . . . . . . . . . . . 144

6.5 Results and Discussion . . . . . . . . . . . . . . 145

6.5 .1 Cascade Inlet Conditions . . . . . . . . . . . . . . 145

6.5 .2 Pressure Probe Results _. . . . . . . . . . . . . . 146

6.5 .3 Flow Visualization Results . . . . . . . . . . . . . 156

6.5 .4 Summary of Results . . . . . . . . . . . . . . . . . . . 159

6.6 Conclusions . . . . . . . . . . . . . . . . . . . . . . 164

7 The Effect of Loading Distribution on Blade Row Loss in Highly-Loaded Linear Cascades with Planar Endwalls $\quad 166$

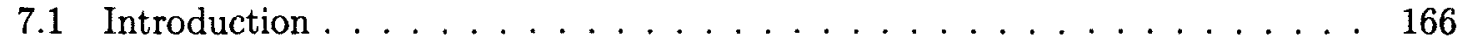

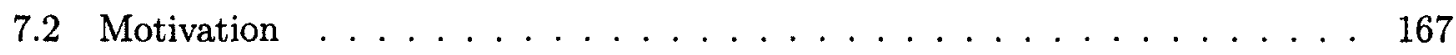

7.3 Approach . . . . . . . . . . . . . . . . . . . 169

7.4 Results and Discussion . . . . . . . . . . . . . . . . . 170

7.4 .1 Velocity Distributions . . . . . . . . . . . . . 170

7.4 .2 Measured Flow Field . . . . . . . . . . . . . . . . . . 172

7.4 .2 .1 Midspan Flow . . . . . . . . . . . . . . . 172

7.4 .2 .2 Endwall Flow . . . . . . . . . . . . . . 175

7.4 .3 Flow Visualization . . . . . . . . . . . . . . . . . . 182 
7.4.4 Comparison of Computational Predictions with Experimental Data . 198

7.4.5 Discussion on Penetration Depth and Secondary Loss Correlation . . 205

7.5 Conclusions . . . . . . . . . . . . . . . . . . . . 208

8 The Effect of Blade Loading on Losses in an Extremely Highly-Loaded Linear Turbine Cascade with Planar Endwalls 211

8.1 Introduction . . . . . . . . . . . . . . . . . 211

8.2 Results and Discussion . . . . . . . . . . . . . . . . . . . . . . 212

8.2.1 Midspan Performance . . . . . . . . . . . . . . . . 213

8.2 .2 Secondary Flow . . . . . . . . . . . . . . . . . . . 221

8.2 .3 Summary of Results . . . . . . . . . . . . . . . . . 223

8.2.4 The Use of High-Lift Airfoils to Expand the Design Envelope . . . . 224

9 Summary, Contributions, Conclusions and Recommendations 229

9.1 Summary . . . . . . . . . . . . . . . . . . . . . 229

9.2 Contributions . . . . . . . . . . . . . . . . . . . 231

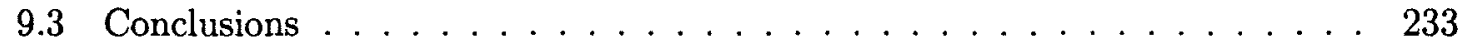

9.4 Recommendations for Future Work . . . . . . . . . . . . . . . . . 236

References $\quad 239$

A Calibration Coefficients for Pressure Transducers 253

B Calibration and Data Reduction Procedures for Three-Hole Pneumatic Probe

C Calibration and Data Reduction Procedures for Seven-Hole Pneumatic Probe

C.1 Low-Angle Regime . . . . . . . . . . . . . . . . . . . . . . . . . 261

C.2 High-Angle Regime . . . . . . . . . . . . . . . . . 268 


\section{List of Tables}

3.1 Cascade nomenclature . . . . . . . . . . . . . . 53

3.2 Cascade geometry . . . . . . . . . . . . . . 53

3.3 Range of inlet boundary layer parameters and mass-averaged $C_{P o} \ldots \ldots$

4.1 Two-dimensional grid sensitivity parameters . . . . . . . . . . 87

4.2 Three-dimensional grid sensitivity parameters . . . . . . . . . . . 97

5.1 Summary of PAKB results . . . . . . . . . . . . . . . . 111

5.2 Effect of endwall contouring at $1.40 B_{x}$, change in measured mass-averaged quantities relative to planar case . . . . . . . . . . . 137

6.1 Summary of PAKD-F results $\ldots \ldots \ldots \ldots \ldots$. . . . . . . . . 147

6.2 Loss breakdown - mixing losses from measurement plane . . . . . . . . . 163

7.1 Summary of experimental planar endwall exit plane results for PAKB, PAKD-A, PAKD-F and PAKD-FP . . . . . . . . . . . . . 173

7.2 Summary of minimum pressure-surface velocity ratio, mixed-out secondary loss and secondary kinetic energy . . . . . . . . . . . . . . 175

7.3 Summary of predicted planar endwall exit plane results for PAKB, PAKD-A, PAKD-F and PAKD-FP . . . . . . . . . . . . . . . 204

7.4 Summary of predicted (and measured) exit plane results, percent change relative to PAKB for PAKD-A, PAKD-F and PAKD-FP . . . . . . . . . . . 204

7.5 Summary of secondary loss, penetration depth and quality factor for four cascades . . . . . . . . . . . . . . . . . . 207 
8.1 Summary of suction surface peak velocity for PAKB, PAKD-A, PAKD-F,

PAKD-FP and PAKF . . . . . . . . . . . . . . 215

8.2 Summary of experimental planar endwall results for PAKB, PAKD-A, PAKD-F, PAKD-FP and PAKF . . . . . . . . . . . . . . 224

A.1 DRAL501DN calibration coefficients . . . . . . . . . . . . . 254

A.2 DRAL505DN calibration coefficients . . . . . . . . . . . . 254 


\section{List of Figures}

1.1 Cut-away of the Pratt \& Whitney PW4000-100 engine . . . . . . 2

1.2 Isometric of non-axisymmetric contoured endwall . . . . . . . . 5

2.1 Endwall flow structures, reproduced from Wang et al. (1997) . . . 13

2.2 Endwall flow visualization in a rotor blade passage - smoke wire inside inlet boundary layer, reproduced from Sieverding and Van Den Bosche (1983) . . . . . . . . . . . . . . . 16

2.3 Schematic representation of natural transition, reproduced from White (1974)) . . . . . . . . . . . . . . . 18

2.4 Schematic representation of a laminar-separation/turbulent-reattachment bubble, reproduced from Zhang (2002) . . . . . . . . . . 20

2.5 Dissipation coefficient for laminar and turbulent boundary layers, reproduced from Denton (1993) $\ldots \ldots \ldots 22$

2.6 Interaction of passage vortex with suction surface boundary layer, reproduced from Benner (2003) . . . . . . . . . . . . 23

2.7 Endwall boundary layer in the presence of a linear cascade, reproduced from Benner (2003) . . . . . . . . . . . . . . 25

2.8 PakD-F data, $1.5 \%$ FSTI, $\Lambda=14[\mathrm{~mm}]$, reproduced from Popović (2005) . . . . . . . . . . . . . . . . . .

2.9 Suction surface oil flow visualization, reproduced from Hodson \& Dominy (1987a) . . . . . . . . . . . . . . . 31

2.10 Main contributors to loss production on the suction surface, reproduced from Popović (2005) . . . . . . . . . . . . .

2.11 Comparison of blade loadings at design incidence $\left(\operatorname{Re}=8.0 \times 10^{4}\right)$, reproduced from Popović (2005) . . . . . . . . . . . . . 
2.12 Axisymmetric endwall geometry, reproduced from Dossena et al. (1999)

2.13 Profiled endwall geometry, reproduced from Harvey et al. (2000)

2.14 Pitchwise-mass-averaged exit flow angle for PAKB (inlet Reynolds number of 126,000 and FSTI=4\%) . . . . . . . . . . . 42

2.15 Pressure surface modifications investigated by Brear et al. (2002b) 47

3.1 Wind-tunnel schematic, reproduced from Benner (2003) . . . . 49

3.2 Variable incidence test section . . . . . . . . . . . . . . . 51

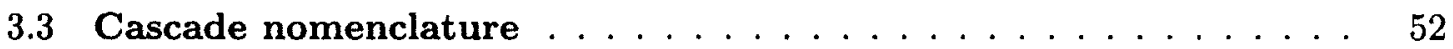

3.4 Research cascades and respective $Z$ Wweifel coefficients . . . . . . 54

3.5 Schematic of probe calibration apparatus, reproduced from Benner

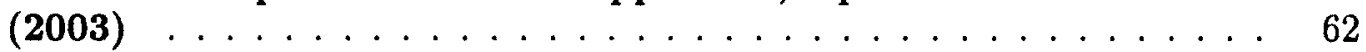

3.6 Three-hole pneumatic probe, reproduced from Benner (2003) . . 66

3.7 Seven-hole pneumatic probe $\ldots \ldots \ldots \ldots$

3.8 Inlet uniformity and outlet periodicity - PAKB . . . . . . . 77

3.9 Total pressure deficit coefficient at inlet plane (a) Pitot-probe and (b) three-hole probe results . . . . . . . . . . . . . . . . . . 79

3.10 Contours of total pressure deficit coefficient overlaid with measurement intervals for PAKB (a \& b) and PAKD-F (c \& d) . . . . . . 80

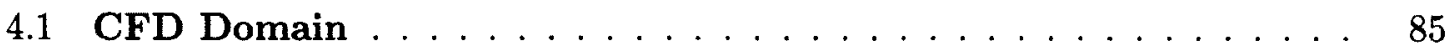

4.2 Schematic representation of blocking $\ldots \ldots \ldots \ldots$

4.3 Relationship between equation residuals and predicted loss coeffi-

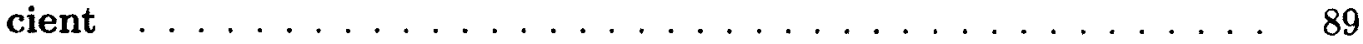

4.4 Predicted and measured mass-averaged loss f . . . . . . . . . . 90

4.5 Predicted and measured mass-averaged flow deviation . . . . . 90

4.6 Midspan wake, predicted and measured total pressure deficit coefficient at $1.20 B_{x} \ldots \ldots \ldots \ldots$. . . . . . . . . . . . . . . 91

4.7 Midspan wake, predicted and measured total pressure deficit coefficient at $1.40 B_{x} \ldots \ldots \ldots \ldots . \ldots \ldots . \ldots \ldots$

4.8 Midspan wake, predicted and measured total pressure deficit coefficient at $1.66 B_{x} \ldots \ldots \ldots \ldots \ldots \ldots$ 
4.9 Predicted and measured midspan loading distribution . . . . . . 93

4.10 Predicted skin friction coefficient $\left(C_{f}\right)$ on suction surface $\ldots \ldots .94$

4.11 Inlet boundary layer profiles for (a) components of velocity and (b) turbulence quantities

4.12 Predicted and measured flood of $C_{\omega_{s}}$ overlaid with line contours of $C_{P_{o}}$ at $1.20 B_{x}$ for grids (a) "3D-G01", (b) "3D-G02", (c) "3D-G03"

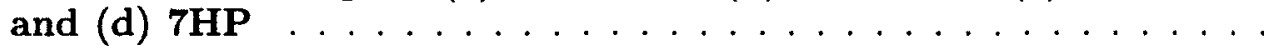

4.13 Predicted and measured mass-averaged (a) $C_{P o}$, (b) $\alpha_{2}$ and (c) $C_{S K E}$

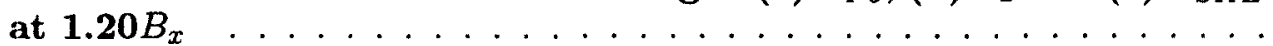

4.14 Predicted and measured flood of $C_{\omega_{s}}$ overlaid with line contours of $C_{P_{0}} 1.40 B_{x}$ for grids (a) "3D-G01", (b) "3D-G02", (c) "3D-G03" and (d) $7 \mathrm{HP} \ldots \ldots \ldots \ldots \ldots$

4.15 Predicted and measured mass-averaged (a) $C_{P_{o}}$, (b) $\alpha_{2}$ and (c) $C_{S K E}$ at $1.40 B$

4.16 Predicted and measured mass-averaged $Y$ at $1.20 B_{x}$ and $1.40 B_{x} \quad . \quad 103$

4.17 (a) Contour plot and (b) slice planes at $z / h=0.50$ and $z / h=0.15$ showing predicted and measured $C_{P o}$ at $1.40 B_{x}$

5.1 Isometric view of PAKB profiled endwall . . . . . . . . . . . . . . 109

5.2 Spanwise variation of total pressure coefficient as measured with a Pitot probe 1.2 Bx upstream of the leading edge at $\mathrm{y} / \mathrm{s}=0.5 \ldots .110$

5.3 Loading distribution measured by Mahallati et al. (2007) . . . . 112

5.4 Endwall (a \& c) and suction surface (b \& d) oil surface flow visualization for the planar (a \& b) and contoured (c \& d) endwall configurations ....................... 114

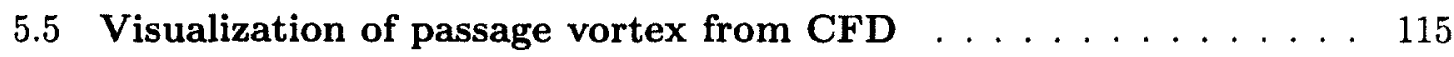

5.6 (a) Line contours of total pressure loss coefficient superimposed with flood of streamwise vorticity and (b) interpreted vortex structure at the trailing edge plane $(1.00 \mathrm{Bx}) \ldots \ldots \ldots$

5.7 Secondary velocity vectors with flood of streamwise vorticity coefficient $\left(C_{\omega s}\right)(\mathrm{a} \& \mathrm{c})$, and line contours of total pressure loss coefficient $\left(C_{P_{0}}\right)$ with flood of secondary kinetic energy coefficient $\left(C_{S K E}\right)(\mathrm{b} \& \mathrm{~d})$, at $63 \%$ axial cord

5.8 Secondary velocity vectors with flood of streamwise vorticity coefficient $\left(C_{\omega s}\right)$ (a \& c), and line contours of total pressure loss coefficient $\left(C_{P_{o}}\right)$ with flood of secondary kinetic energy coefficient $\left(C_{S K E}\right)(\mathrm{b} \& \mathrm{~d})$, at $71 \%$ axial cord 
5.9 Secondary velocity vectors with flood of streamwise vorticity coefficient $\left(C_{\omega s}\right)$ (a \& $\left.\mathbf{c}\right)$, and line contours of total pressure loss coefficient $\left(C_{P_{0}}\right)$ with flood of secondary kinetic energy coefficient $\left(C_{S K E}\right)(\mathbf{b} \& \mathbf{d})$, at $\mathbf{8 0 \%}$ axial cord $\ldots \ldots \ldots$

5.10 Secondary velocity vectors with flood of streamwise vorticity coefficient $\left(C_{\omega s}\right)(\mathrm{a} \& \mathrm{c})$, and line contours of total pressure loss coefficient $\left(C_{P o}\right)$ with flood of secondary kinetic energy coefficient $\left(C_{S K E}\right)(\mathbf{b} \& \mathbf{d})$, at the trailing edge plane $\ldots \ldots \ldots$

5.11 Secondary velocity vectors with flood of streamwise vorticity coefficient $\left(C_{\omega s}\right)$ (a \& $\mathbf{c}$ ), and line contours of total pressure loss coefficient $\left(C_{P_{0}}\right)$ with flood of secondary kinetic energy coefficient $\left(C_{S K E}\right)$ (b \& d), at $140 \%$ axial cord . . . . . . . . . . . .

5.12 Pitchwise mass-averaged results for the $140 \% B_{x}$ measurement plane; Total pressure loss coefficient (a), outlet flow angle (b) and secondary kinetic energy coefficient $(c) \ldots \ldots \ldots$

5.13 Predicted distributions of blade surface static pressure at various spanwise locations . . . . . . . . . . . . . . . . . . .

5.14 Comparison of experimental and predicted endwall flow visualizations for (a \& b) planar and (c \& d) contoured configurations . . .

5.15 Comparison of experimental and predicted suction surface flow visualizations for (a \& b) planar and (c \& d) contoured endwall configurations . . . . . . . . . . . . . . . .

5.16 Measured and predicted secondary velocity vectors with flood of streamwise vorticity coefficient $\left(C_{\omega s}\right)(\mathrm{a} \& \mathrm{c})$, and line contours of total pressure loss coefficient $\left(C_{P o}\right)$ with flood of secondary kinetic energy coefficient $\left(C_{S K E}\right)$ (b \& d), at $140 \%$ axial cord . . . . . . .

5.17 Measured and predicted pitchwise mass-averaged results for the $140 \% B_{x}$ plane; Total pressure loss coefficient (a), outlet flow angle (b) and secondary kinetic energy coefficient (c) . . . . . . . .

6.1 Isometric view of PAKD-F profiled endwall $\ldots \ldots \ldots \ldots \ldots$

6.2 Spanwise variation of total pressure coefficient as measured with a pitot probe $120 \%$ axial chord upstream of the leading edge at $y / s$ $=0.25,0.5$ and 0.75 . . . .

6.3 Secondary velocity vectors with flood of streamwise vorticity coefficient $\left(C_{\omega s}\right)$ (a \& c), and line contours of total pressure loss coefficient $\left(C_{P o}\right)$ with flood of secondary kinetic energy coefficient $\left(C_{S K E}\right)(\mathrm{b} \& \mathrm{~d})$, at $63 \%$ axial cord $\ldots \ldots \ldots . \ldots . \ldots 148$ 
6.4 Secondary velocity vectors with flood of streamwise vorticity coefficient $\left(C_{\omega s}\right)$ (a \& c), and line contours of total pressure loss coefficient $\left(C_{P o}\right)$ with flood of secondary kinetic energy coefficient $\left(C_{S K E}\right)$ (b \& d), at $\mathbf{8 0 \%}$ axial cord $\ldots \ldots \ldots \ldots \ldots \ldots$

6.5 Secondary velocity vectors with flood of streamwise vorticity coefficient $\left(C_{\omega s}\right)(\mathrm{a} \& \mathrm{c})$, and line contours of total pressure loss coefficient $\left(C_{P_{o}}\right)$ with flood of secondary kinetic energy coefficient $\left(C_{S K E}\right)(\mathbf{b} \& \mathbf{d})$, at the trailing edge plane $\ldots \ldots \ldots \ldots \ldots$

6.6 Secondary velocity vectors with flood of secondary kinetic energy coefficient $\left(C_{S K E}\right)$ (a \& c), and line contours of total pressure loss coefficient $\left(C_{P_{0}}\right)$ with flood of streamwise vorticity coefficient $\left(C_{\omega s}\right)$ (b \& d), at $120 \%$ axial cord . . . . . . . . . . . . . . . .

6.7 Secondary velocity vectors with flood of secondary kinetic energy coefficient $\left(C_{S K E}\right)$ (a \& c ), and line contours of total pressure loss coefficient $\left(C_{P o}\right)$ with flood of streamwise vorticity coefficient $\left(C_{\omega s}\right)$ (b\& d), at $140 \%$ axial cord $\ldots \ldots \ldots \ldots \ldots \ldots \ldots$

6.8 Planar endwall oil surface flow visualization showing a) endwall b) blade suction surface c) suction surface - endwall junction . . . . .

6.9 Contoured endwall oil surface flow visualization showing a) endwall b) blade suction surface $\ldots \ldots \ldots \ldots \ldots \ldots \ldots$

6.10 Pitchwise mass-averaged results for the $140 \% B_{x}$ measurement plane (a) total pressure loss coefficient, (b) outlet flow angle and (c) secondary kinetic energy coefficient $\ldots \ldots \ldots \ldots$

6.11 Contributions to mixed-out loss $\ldots \ldots \ldots \ldots \ldots \ldots$

7.1 Predicted velocity distributions for PAKB, PAKD-A, PAKD-F and

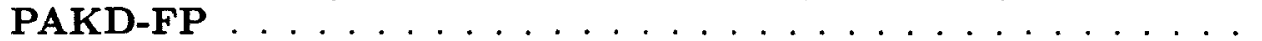

7.2 Total pressure deficit coefficient for the midspan wakes of PAKDA, PAKD-F and PAKD-FP as measured at the exit plane . . . .

7.3 Secondary velocity vectors with flood of $C_{S K E}(\mathrm{a}, \mathrm{b}, \mathrm{c} \& \mathrm{~d})$, and line contours of $C_{P o}$ with flood of $C_{H e}(e, f, g \& ~ h)$, at $80 \%$ axial cord for PAKB, PAKD-A, PAKD-F and PAKD-FP . . . . . .

7.4 Secondary velocity vectors with flood of $C_{S K E}(\mathrm{a}, \mathrm{b}, \mathrm{c} \& \mathrm{~d})$, and line contours $C_{P o}$ with flood of $C_{H e}(\mathrm{e}, \mathrm{f}, \mathrm{g} \& \mathrm{~h})$, at the trailing edge plane for PAKB, PAKD-A, PAKD-F and PAKD-FP . . . .

7.5 Secondary velocity vectors with flood of $C_{S K E}(\mathrm{a}, \mathrm{b}, \mathbf{c} \& \mathrm{~d})$, and line contours of $C_{P o}$ with flood of $C_{\omega s}(e, f, g \& h)$, at $120 \%$ axial cord for PAKB, PAKD-A, PAKD-F and PAKD-FP 
7.6 Secondary velocity vectors with flood of $C_{S K E}(\mathrm{a}, \mathrm{b}, \mathrm{c} \& \mathrm{~d})$, and line contours of $C_{P_{o}}$ with flood of $C_{\omega s}(\mathrm{e}, \mathrm{f}, \mathrm{g} \& \mathrm{~h})$, at $140 \%$ axial cord for PAKB, PAKD-A, PAKD-F and PAKD-FP . . . . . . . 183

7.7 Endwall flow visualization for PAKB, PAKD-A, PAKD-F and PAKD-FP (Experiment vs. CFD) . . . . . . . . . . . . . . 185

7.8 Suction surface flow visualization for PAKB, PAKD-A, PAKD-F and PAKD-FP (Experiment vs. CFD) . . . . . . . . . . . 187

7.9 Predicted streamlines from $y+=4$ for PAKD-F (a \& b) and PAKDFP $(c \& d) \ldots \ldots \ldots \ldots 1 . \ldots \ldots \ldots$

7.10 Predicted streamlines from the overlap region for PAKD-F ( $a, b$ $\& \mathrm{c})$ and PAKD-FP (d, e \& f)

7.11 Predicted streamlines from $y+=2024$ for PAKD-F (a \& b) and PAKD-FP $(\mathbf{c} \& \mathbf{d})) \ldots \ldots \ldots \ldots$

7.12 Experimental suction surface flow visualization for PAKD-FP . . . 197

7.13 Line contours of total pressure loss coefficient $\left(C_{P_{0}}\right)$ with flood of streamwise vorticity coefficient $\left(C_{\omega s}\right)$, Experiment $(\mathbf{a}, \mathbf{b}, \mathbf{c} \& \mathbf{d})$ vs. CFD (e, f, g \& h), at $120 \%$ axial cord for PAKB, PAKD-A, PAKD-F and PAKD-FP . . . . . . . . . . . . .

7.14 Line contours of total pressure loss coefficient $\left(C_{P_{0}}\right)$ with flood of streamwise vorticity coefficient $\left(C_{\omega s}\right)$, Experiment $(\mathrm{a}, \mathrm{b}, \mathbf{c} \& \mathrm{~d})$ vs. CFD (e, f, $g \& \mathrm{~h}$ ), at $140 \%$ axial cord for PAKB, PAKD-A, PAKD-F and PAKD-FP . . . . . . . . . . . . . . .

7.15 Measured and predicted pitchwise mass-averaged results for the $140 \% B_{x}$ plane; Total pressure loss coefficient (a), pitchwise flow angle (b) and secondary kinetic energy coefficient (c) . . . . . . .

8.1 Computed loading distribution with experimental validation for five planar cascades, $R e=1.26 \times 10^{5} \ldots \ldots \ldots \ldots 213$

8.2 Velocity ratio along suction surface, $R e=1.26 \times 10^{5} \ldots \ldots \ldots 214$

8.3 Mixed-out midspan flow angle vs. Reynolds number . . . . . . . 217

8.4 Characteristics of wakes with Reynolds number variation for PAKF at $140 \%$ axial cord . . . . . . . . . . . . . . 218

8.5 Mixed-out profile loss vs. Reynolds number . . . . . . . . . . . 220

8.6 Line contours of $C_{P o}$ with flood of $C_{\omega s}$, at $140 \%$ axial cord for

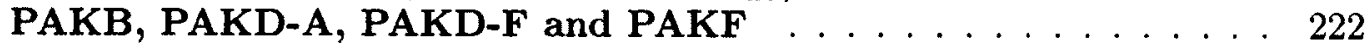

8.7 Secondary loss vs. Zweifel coefficient and the use of high-lift airfoils 226 
A.1 Sample calibration curves for DRAL501DN pressure transducers . 254

A.2 Sample calibration curves for DRAL505DN pressure transducers . 255

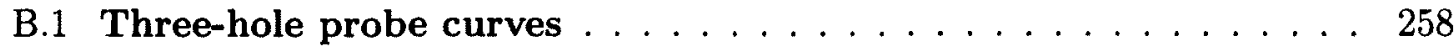

C.1 Division of angular space (reproduced from Gerner et al. (1984) . 261

C.2 Tangential coordinate system for low-angle regime (reproduced from Benner, 2003) . . . . . . . . . . . . . . . . . 262

C.3 $C_{\alpha}$ calibration data for sector $7 \ldots \ldots \ldots \ldots$

C.4 $C_{\beta}$ calibration data for sector $7 \ldots \ldots \ldots \ldots$

C.5 $C_{o}$ calibration data for sector $7 \ldots \ldots \ldots \ldots$

C.6 $C_{q}$ calibration data for sector $7 \ldots \ldots \ldots \ldots$

C.7 Cylindrical coordinate system for high-angle regime (reproduced from Benner, 2003) . . . . . . . . . . . . . . . . 268

C.8 $C_{\phi}$ calibration data for sector $4 \ldots \ldots \ldots \ldots \ldots$

C.9 $C_{\theta}$ calibration data for sector $4 \ldots \ldots \ldots \ldots$

C.10 $C_{o}$ calibration data for sector $4 \ldots \ldots \ldots \ldots 272$

C.11 $C_{q}$ calibration data for sector $4 \ldots \ldots \ldots \ldots \ldots$ 


\section{List of Symbols}

$\begin{array}{ll}A V R & \text { axial velocity ratio }=\frac{u_{M S_{2}}^{\prime}}{u_{M S_{1}}^{\prime}} \\ A_{t p} & \text { area of traverse plane } \\ B_{x} & \text { axial chord } \\ C & \text { true chord length } \\ C R & \text { convergence ratio }=\text { cos } \alpha_{1} / \cos \alpha_{2} \\ C_{f} & \text { skin friction coefficient }=\tau_{w a l l} / q_{1}^{\prime} \\ C_{H_{e}} & \text { non-dimensional coefficient of helicity }=\frac{H_{e} C}{V_{C L}^{2}} \\ C_{P} & \text { static pressure coefficient }=\frac{P-P_{C L_{1}}}{q_{C L_{1}}} \\ C_{P-L} & \text { blade loading static pressure coefficient }=\frac{P o_{C L_{1}}-P}{q_{C L_{1}}} \\ C_{P o} & \text { total pressure coefficient }=\frac{P o-P o_{C L_{1}}}{q_{C L_{1}}} \\ C_{P o_{p r o f i l e}} & \text { profile pressure loss coefficient }=\frac{P o_{C L_{2}}-P o_{C L_{1}}}{q_{C L_{1}}} \\ C_{P o_{s e c o n d a r y}} & \text { secondary loss coefficient }=C_{P o_{t o t a l}}-C_{P o_{p r o f i l e}} \\ C_{P o_{\text {total }}} & \text { total pressure loss coefficient }=\frac{P o_{2}^{\prime \prime}-P o_{1}^{\prime \prime}}{q_{C L_{1}}} \\ C_{q} & \text { dynamic pressure coefficient }=\frac{P o-P}{q_{C L_{1}}}=1+C_{P o}-C P \\ C_{S K E} & \text { nondimensional secondary kinetic energy }=S K E / q_{C L_{1}} \\ C_{\omega_{s}} & \text { nondimensional streamwise vorticity }=\omega_{s} \cdot C / V_{C L} \\ & \end{array}$


deceleration parameter $=\frac{V_{\max } / V_{2}}{\left(\frac{\left(1-s^{\dagger}\right) \cdot S_{\max }}{B_{x}}\right)}$

$d$

diameter of calibration nozzle

$F_{y}$

tangential force $=\frac{2 \cdot A_{t p} \cdot \bar{u}}{q_{C L_{1} \cdot B_{x}^{2}}}\left[\left(u \cdot \tan \alpha_{1}\right)^{\prime \prime}-\left(u \cdot \tan \alpha_{2}\right)^{\prime \prime}\right]$

H

boundary layer shape factor $=\delta^{*} / \theta$

$H_{e} \quad$ helicity $=V \cdot(\nabla \times V)=u \omega_{x}+v \omega_{y}+w \omega_{z}$

$h$

blade span

$k$

turbulent kinetic energy

$R e$

Reynolds number $=\rho V_{C L} C / \mu$

$R e_{v}$

vorticity Reynolds number $=\frac{\rho y^{s}}{\mu} \frac{\partial u}{\partial y}==\frac{\rho y^{s}}{\mu} \Omega$

$R e_{\theta} \quad$ momentum thickness Reynolds number

$R e_{\theta t} \quad$ transition onset momentum thickness $R e$ (based on freestream conditions) $=\frac{\rho \theta_{t} U}{\mu}$

$P \quad$ static pressure

$P_{0} \quad$ total pressure

$Q$

quality factor $=\left(\Delta C p_{\max } \frac{q_{C L}}{q_{\text {mean }}} \frac{B_{x}}{s^{*}}\right)\left(1-\frac{x_{\min }}{B_{x}}\right)$

$q$

dynamic pressure $P_{o}-P=1 / 2 \rho V^{2}$

$q_{\text {mean }}$

vector mean of inlet and outlet dynamic pressure

$S$

separation streamline, surface length

$S / S_{\max } \quad$ fraction of surface length

$S P \quad$ suction peak

$S K E \quad$ secondary kinetic energy $=1 / 2 \rho\left(v_{s e c}^{2}+w_{s e c}^{2}\right)$

$s$

blade pitch

$s^{*}$

cross passage distance at location of suction peak (see Quality),

also fraction of surface length $\left(S / S_{\max }\right)$

TE trailing edge

$u$

axial component of velocity 


\begin{tabular}{|c|c|}
\hline$\tilde{u}$ & area-averaged axial velocity \\
\hline$u_{\tau}$ & friction velocity $=\sqrt{\frac{\tau_{w}}{\rho}}$ \\
\hline$V$ & velocity \\
\hline$v$ & pitchwise / circumferential component of velocity \\
\hline$v_{s e c}, w_{s e c}$ & normal components of the secondary velocity vector \\
\hline$w$ & spanwise / radial coordinate \\
\hline$x$ & axial coordinate \\
\hline$x_{\min }$ & axial coordinate of suction peak \\
\hline$Y_{\text {profile }}$ & mass-averaged profile loss coefficient $=\frac{C_{P_{o_{1}}}^{\prime}-C_{P_{o_{2}}}^{\prime}}{q_{2}^{\prime}}$ \\
\hline$Y_{\text {secondary }}$ & mass-averaged secondary loss coefficient $=Y_{\text {total }}-Y_{\text {profile }}$ \\
\hline$Y_{t i p}$ & mass-averaged tip leakage loss coefficient \\
\hline$Y_{\text {total }}$ & mass-averaged total pressure loss coefficient $=\frac{C_{P_{o_{1}}}^{\prime \prime}-C_{P_{o_{2}}}^{\prime \prime}}{q_{2}{ }^{\prime \prime}}$ \\
\hline$y$ & pitchwise / circumferential coordinate \\
\hline$y^{+}$ & non-dimensional wall distance for wall-bounded flow $=\frac{\rho \cdot \Delta y \cdot u_{\tau}}{\mu}$ \\
\hline$Z_{T E}$ & penetration height of secondary flow \\
\hline$z$ & spanwise / radial coordinate \\
\hline$\alpha$ & pitchwise flow angle \\
\hline$\gamma$ & stagger angle \\
\hline$\Delta y$ & height from wall for wall-bounded flow \\
\hline$\Delta C p_{\max }$ & maximum non-dimensional cross passage pressure difference \\
\hline$\delta$ & boundary layer thickness \\
\hline$\delta^{*}$ & displacement thickness \\
\hline$\epsilon$ & turbulence eddy dissipation \\
\hline$\Theta$ & momentum thickness \\
\hline$\Lambda$ & turbulence length scale \\
\hline$\mu$ & dynamic viscosity \\
\hline
\end{tabular}


momentum thickness

$\rho$

density

$\tau_{w}$

wall shear stress

$\omega$

turbulent frequency

$\omega_{s}$

streamwise vorticity $=\omega_{x} \cos \alpha^{\prime \prime}+\omega_{y} \sin \alpha^{\prime \prime}$

$\omega_{x}$

$\mathrm{x}$-component of vorticity $=\frac{\partial w}{\partial y}-\frac{\partial v}{\partial z}$

$\omega_{y}$

$\mathrm{y}$-component of vorticity $=\frac{\partial u}{\partial z}-\frac{\partial w}{\partial x} \approx \frac{1}{u}\left(v \omega_{x}+\frac{1}{\rho} \frac{\partial P_{o}}{\partial z}\right)$

$\omega_{z}$

z-component of vorticity $=\frac{\partial v}{\partial x}-\frac{\partial u}{\partial y} \approx \frac{1}{u}\left(w \omega_{x}-\frac{1}{\rho} \frac{\partial P_{o}}{\partial y}\right)$

\section{Subscripts}

1,2

cascade inlet and outlet, respectively

$C L \quad$ midspan value mass-averaged over one blade pitch

des design

e exit

$l \quad$ local

local local

MS midspan

$P \quad$ pressure leg of the separation streamlines

peak peak

$S \quad$ suction leg of the separation streamlines

$t \quad$ turbulent, or transition

\section{Superscripts}

1

pitchwise mass-averaged value

II

mass-averaged value 


$\begin{array}{ll}\text { Abbreviations } \\ \text { 3HP } & \text { three-hole probe } \\ \text { 7HP } & \text { seven-hole probe } \\ \text { AC } & \text { alternating current } \\ \text { FSTI } & \text { freestream turbulence intensity } \\ \text { H } & \text { hill } \\ \text { HP } & \text { horse power } \\ \text { LSTL } & \text { low-speed turbomachinery laboratory } \\ \text { LPT } & \text { low-pressure turbine } \\ \text { PWA } & \text { Pratt and Whitney Aircraft } \\ \text { R } & \text { reattachment } \\ \text { RANS } & \text { Reynolds-averaged Navier-Stokes } \\ \text { S } & \text { separation } \\ \text { SST } & \text { shear stress transport (turbulence closure model) } \\ \text { USB } & \text { universal serial bus } \\ \text { V } & \text { valley }\end{array}$




\section{Chapter 1}

\section{Introduction}

\subsection{Motivation}

The goal of the aero engine manufacturer is the design of cost-effective engines for thrust generation. This entails the development of engines appropriately sized to meet the needs of the customer at a minimum expense. When purchasing an engine, the customer must consider both the initial capital cost of the unit, as well as its life cycle costs. It is often the latter that are of primary concern. Engines tasked with continuous or near continuous operation can reach life cycle costs orders of magnitude greater than their initial purchase price. The term 'life cycle cost' is used to represent maintenance costs, and fuel costs that account for the bulk of the expense. In consequence of this, the efficiency of the engine is of principal consideration during design.

Figure 1.1 shows the key components of the Pratt \& Whitney PW4000-100 highbypass turbo-fan engine. This particular engine is used to power the Airbus 330 family of aircraft. The PW4000 series engines have a twin spool design. The 2.54 meter fan and the low-pressure compressor are driven by the low-pressure turbine while the high-pressure 
turbomachinery operate on a separate concentric shaft. The efficiency of these components has reached a high level and significant performance gains have become difficult to achieve. As a result designers have begun to look for other methods of improving designs to gain a competitive edge. This has led to efforts aimed at weight and part count reductions while maintaining current levels of efficiency.

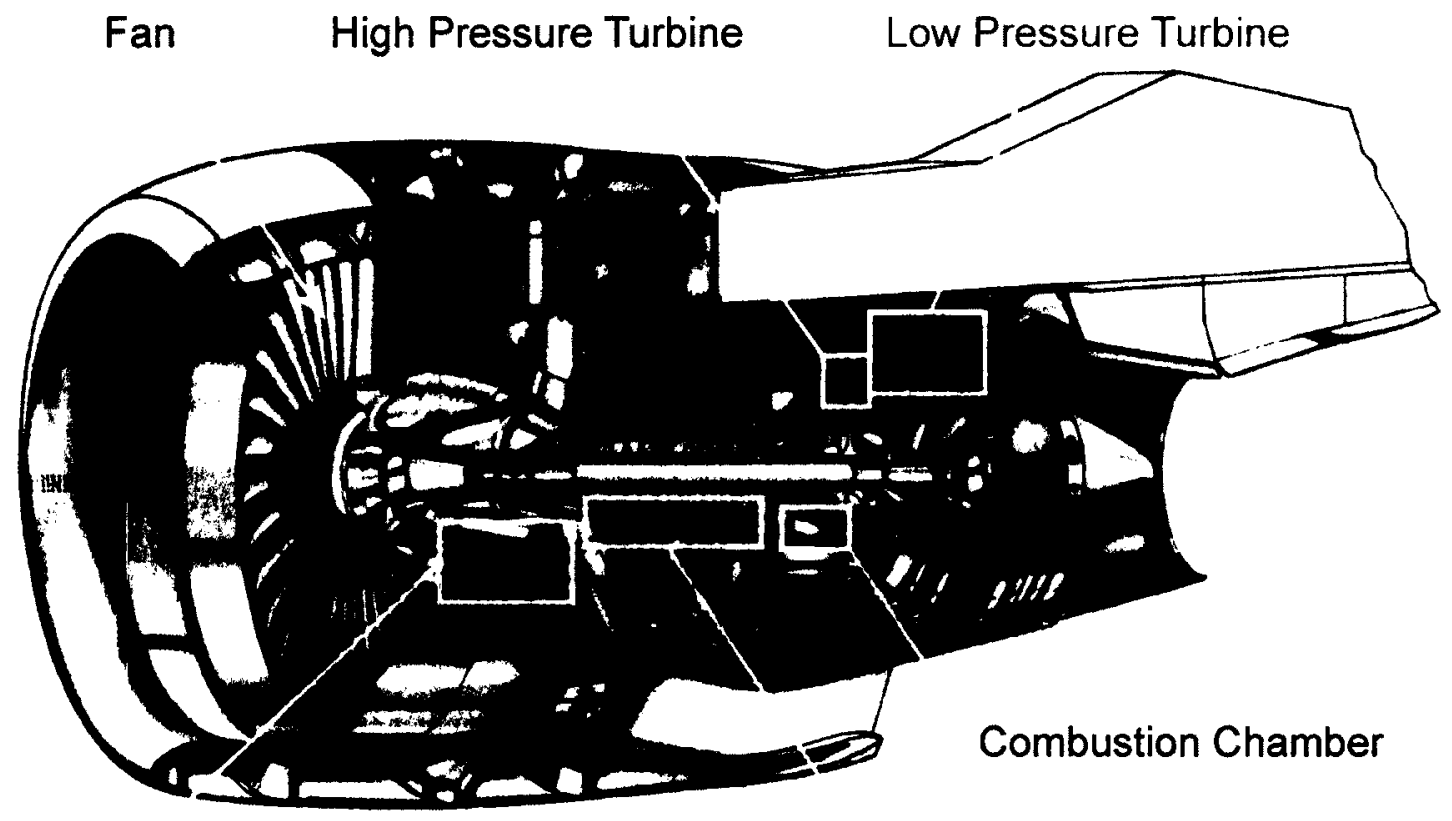

Low Pressure Compressor

High Pressure Compressor

Figure 1.1: Cut-away of the Pratt \& Whitney PW4000-100 engine

The fan of the PW4000-100 operates at a pressure ratio of about 1.75 and generates roughly $80 \%$ of the engine's 68,600 pounds of thrust. In order to generate the power required to drive the fan and the low-pressure compressor, a multiple stage low-pressure turbine (LPT) is required. The LPT of a modern high-bypass-ratio turbofan engine can account for as much as one third of the total engine weight (Curtis et al., 1997). This makes the LPT an obvious target for weight reduction. Engine weight (along with manufacturing and repair costs) can be decreased by reducing the number of blades per stage. This effectively increases the aerodynamic duty of each blade and could have subsequent performance 
penalties associated with the profile performance as well as the highly three-dimensional flow structures in the endwall region. As such, highly loaded stages would be unattractive from an efficiency stand point.

The research group at Carleton has been investigating the aerodynamics of highlyloaded low-pressure turbine blades for a number of years (Mahallati, 2003; Popović, 2005; Zorić, 2006). Initial studies focussed on the two dimensional flow around the blade profiles, away from the endwalls. The investigations examined a family of airfoils with aerodynamic loadings varying from approximately the levels seen in current aero engines to nearly twice those levels. The results demonstrated that it is possible to design airfoils with moderate losses and good stall characteristics to loading levels much higher than current design practice. Such designs would allow a significant reduction in the number of airfoils in a given blade row, thus reducing its weight and manufacturing costs.

However, the measurements also showed that the higher loading levels strengthened the secondary flows near the endwalls and thus increased the secondary losses (Zorić, 2006). This was not unexpected since investigations by other researchers had also shown stronger secondary flows with higher loading levels (Hodson and Dominy, 1987a; Perdichizzi and Dossena, 1993). Unfortunately, this was particularly true for the front-loaded designs, which had the most advantageous profile flow characteristics (Popovic et al., 2006; Zorić et al., 2007a). Thus, to take advantage of the higher loadings it is necessary to devise design strategies that mitigate the accompanying rise in secondary losses. The present work has investigated two such design strategies. These design strategies have been developed partly on the basis of the physical insights into the endwall flow behaviour obtained from the earlier studies conducted at Carleton (eg. Zorić, 2006)

Secondary flows and the accompanying loss mechanisms will be described more fully in Chapter 2. Briefly, the secondary flows are the result of the blade-to-blade pressure 
differences which drive the lower momentum fluid near the endwalls from the high pressure side ("pressure side") of the blade passage towards the low pressure side ("suction side"). This flow typically rolls up into one or more vortices and, in general, the stronger the vortical structures the higher the secondary losses. Thus, to lower the losses it is necessary to weaken the secondary flows or otherwise impede their formation.

\subsection{Approach}

Two approaches to accomplishing this have been examined: non-axisymmetric endwall contouring and modification to the blade loading distributions.

The first technique, non-axisymmetric endwall contouring, consists of modifying the blade-passage endwall geometry in both the axial and tangential directions, as shown in Figure 1.2. The contouring affects the local static pressure on the endwall by means of streamline curvature. A convex curvature relative to the nominal endwall height locally accelerates the flow and reduces the local static pressure. A concave wall curvature causes a relative deceleration of the flow, thereby raising the local static pressure. It has been shown that the flow physics can be altered in a beneficial manner by modifying the static pressure near the endwall (Hartland et al., 2000; Rose et al., 2001).

Unlike endwall contouring, which has received widespread attention, the second approach is somewhat novel (and is the subject of a patent filing by Pratt \& Whitney Aircraft). It originates in the observation that the lower the streamwise momentum of a flow, the more strongly it will be driven in the transverse direction by a pressure gradient in that direction. Thus, a turbine airfoil that has a large region of near-stagnation pressure on the pressure surface will provide a large mass of low momentum fluid that will be strongly swept across the passage to form a strong secondary flow. The detailed pressure distribution around a 


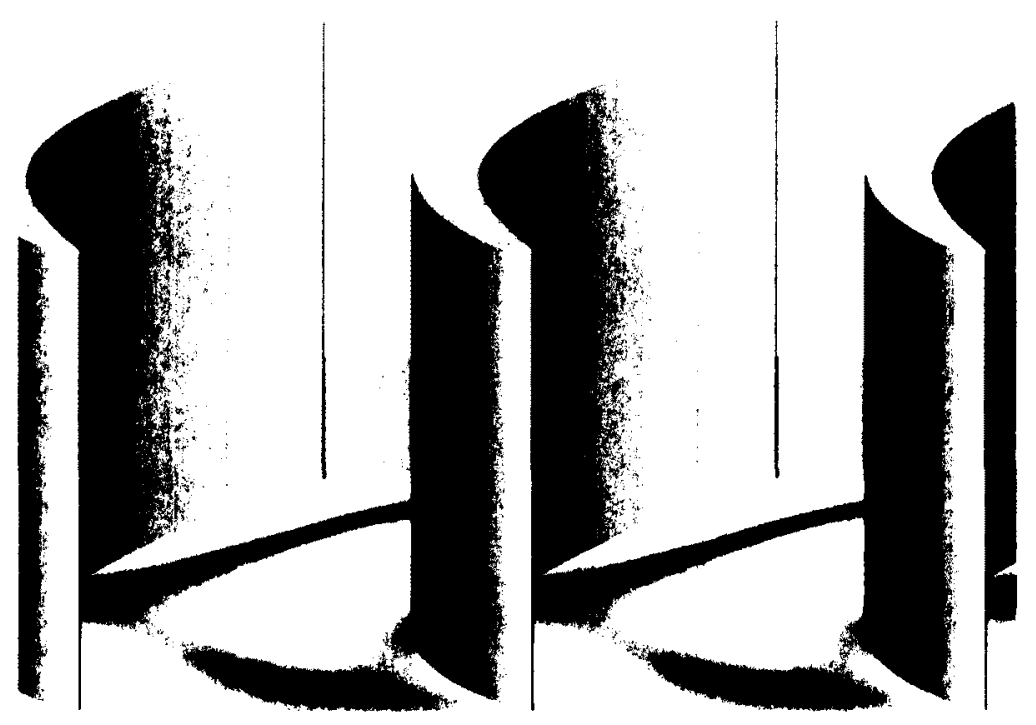

Figure 1.2: Isometric of non-axisymmetric contoured endwall

turbine blade is controlled by the designer through the profile geometry. Therefore, it is possible to reduce the extent of the high-pressure region on the pressure side of the airfoil. The lower pressures on the pressure side must of course be off-set by lower pressures on the suction side, to maintain the overall pressure difference across the airfoil and thus its lift. Consequently, the pressure difference driving the endwall flow is essentially unchanged. However, it will be shown that the generally higher streamwise momentum of the fluid driven by this pressure difference results in a weaker cross-passage flow, weaker secondaryflow structures and thus lower secondary losses. The modified-loading approach has the advantage that it does not require the complex endwall geometry, and therefore higher manufacturing cost, of the contoured endwalls.

Both passive flow control techniques were tested experimentally at Carleton University's Low-Speed Turbomachinery Laboratory using a linear cascade test section. Pneumatic probes are used as the primary measurement technique for quantifying loss and flow direction. Measurements are taken within the blade passage and downstream of the trailing 
edge to track the progression of the various vortical structures that comprise the endwall flow. The probe measurements are supplemented with oil surface flow visualizations on the endwall and blade suction surface. The flow visualizations can be used to interpret the direction and magnitude of the surface shear stress, and the location of the boundary layer separation and reattachment lines give insights into the flow physics. The experimental and data reduction procedures are described in Chapter 3.

Complementary computational fluid dynamics (CFD) simulations were conducted where they were deemed appropriate. The simulations were performed using the ANSYS-CFX commercial software package (versions 5.7.1, 10.0, and 11.0). The results of the simulations are used to enhance the understanding of the flow physics inferred from the experimental measurements. The details of the numerical method and validation of the computations is presented in Chapter 4.

The test matrix examines a family of aerofoils having the same inlet and outlet velocity triangles, designed by Pratt \& Whitney Aircraft (PWA). The family of airfoils have a systematic variation in blade loading and loading distribution which make them a useful vehicle for the studying endwall flows. The foundation of this family is the PAKB aerofoil, representative of the midspan cross section of a PWA low-pressure turbine blade. PAKB has been tested extensively at Carleton University (Mahallati, 2003; Zhang, 2002; McAuliffe, 2003). The role of PAKB in the current study is to act as a benchmark for the subsequent geometries.

PAKD-F and PAKD-A are the next generation derivatives of PAKB. The ' $F$ ' signifies a forward loading (the suction peak occurs between between the leading edge and mid axial chord), while the 'A' signifies aft loading. The PAKD airfoils have $25 \%$ higher loading than PAKB. These airfoils were examined by Zorić (2006) and Popović (2005) and the results of these studies demonstrated the attractiveness of front-loading as discussed above. 
It is noteworthy that the aft-loaded PAKD-A produces lower secondary losses than those of PAKD-F at the design condition but has inferior profile performance, stalling at offdesign incidence or reduced Reynolds number. As a result, the PAKD-F geometry is of primary focus in the present study. It has been tested with a non-axisymmetric endwall, and has been re-designed and tested implementing the modified-loading approach. During the redesign, the pressure-side of the airfoil was modified as described earlier to reduce the extent of near-stagnation flow on that side. Small modifications were also made to the suction side, but the pressure distribution closely matches that of PAKD-F. The redesigned airfoil carries the designation PAKD-FP.

Additionally, a fifth aerofoil has been designed during the course of the current study. This airfoil, designated PAKF is front-loaded and has a loading level $65 \%$ higher than that of PAKB. It is intended to push the limits of blade loading and to demonstrate the predictive capability of the transition model used for its design (Praisner and Clark, 2007a). Remarkably, Praisner et al. (2008) showed that PAKF has similar profile performance to PAKB despite having the highest loading of any linear turbine cascade documented in the open literature. This further reinforces the notion that forward-loading could potentially be used in conjunction with secondary flow control techniques to implement high-lift designs.

In the current study the PAK family of airfoils are used to investigate the generation of loss in the endwall region of highly loaded turbine blade passages and to explore the effectiveness of passive flow control techniques described above for the reduction of loss.

\subsection{Thesis Objectives}

The work presented herein is both fundamental and applied in nature. The fundamental focus of the research is to develop a better understanding of the flow field around ultra- 
highly-loaded turbine aerofoils in the absence of tip clearance. The primary interest is the evolution of the three-dimensional vortical structures near the endwall and their associated losses. This includes developing insights into the mechanisms of entropy generation in the endwall region. It was mentioned above that previous authors have shown endwall contouring to be an effective means to reduce secondary losses, but the mechanisms for loss reduction are not completely understood. The current work focuses on changes to the physics of the endwall flow that result from geometric modifications and how they affect loss.

The applied nature of the work takes two forms. First, the body of data generated is used as a validation tool for computational fluid dynamics (CFD) simulations and to assess the capability of predicting loss reduction resulting from subtle geometric modifications. Secondly, the various test cases demonstrate the ability to increase blade loading beyond that of current industry design practices with little or no performance penalties in the endwall regions using passive flow control techniques.

Since the test matrix is comprised of several airfoils and a series geometric modifications, each of the results chapters are prefaced with specific additional objectives.

\subsection{Overview of Thesis}

A substantial amount of research regarding endwall losses in axial flow turbines has been conducted and the field is considered to be in a mature state. A review of the pertinent literature is given in Chapter 2. The physics of the endwall flow is described in detail and the relationship between loss generation and various aerodynamic and geometric parameters is discussed. The chapter concludes with a survey of endwall contouring design techniques and a review of experimental results obtained by other researchers. 
The experimental facility and measurement techniques used in the present work are described in Chapter 3. The Carleton University low-speed turbomachinery wind tunnel and linear cascade test section are described first, followed by the measurement system and techniques. The details of the cascades used in the study are given along with wind tunnel operating procedures. The chapter includes an outline of the data reduction procedures and a discussion of measurement uncertainties.

Chapter 4 describes the numerical setup and procedures for the computational portion of the research. The numerical methods implemented in the ANSYS-CFX solver are outlined including discretization, boundary-condition specifications, and convergence criteria. The chapter focuses on the grid independence of the computational domains used in the study and contains some validating comparisons to data taken experimentally.

The chapters which follow present the results of the various studies. Chapter 5 describes an experimental assessment of the performance of non-axisymmetric endwall contouring for the baseline cascade, PAKB. The chapter examines the flow field through the blade passage with a planar endwall, and compares it to the case where endwall contouring has been applied. The focus is on developing a better understanding of the flow physics and the relationship to loss generation using pressure measurements and oil surface flow visualization. Complementary CFD simulations for both the planar and contoured endwall configurations demonstrate the capabilities and shortfalls of commercial software for predicting the three-dimensional secondary flows.

Chapter 6 presents experimental data for the more highly- and front-loaded PAKD-F cascade both with and without the implementation of endwalls contouring. The contouring is successful in reducing endwall loss to a level near that of the baseline airfoil PAKB. This chapter demonstrates the potential feasibility of high-lift front-loaded airfoils if used in conjunction with endwall profiling for secondary loss mitigation. The chapter also contains 
a detailed breakdown of the contributions to mixed-out loss and elaborates on the effect of secondary kinetic energy on loss generation.

The results for the second strategy for endwall flow control, namely blade loading modification, are presented in Chapter 7. Experimental and computational avenues are used to compare the flow field of PAKD-FP to those of PAKD-F, PAKD-A and PAKB with planar endwalls. The chapter shows that modifying the velocity distribution around the airfoil can achieve similar benefits to endwall contouring without added manufacturing complexity. This again shows the plausible effectiveness of forward-loading for high-lift low-pressure turbine design. The chapter also demonstrates the capabilities and inabilities of commercial software for predicting loss generation and secondary flow physics for linear cascades with planar endwalls.

Chapter 8 examines the flow through a cascade of extremely highly-loaded PAKF airfoils. As discussed above, this remarkable airfoil has the highest loading level of any linear turbine cascade documented in the open literature. The profile performance of the cascade is documented experimentally over a range of Reynolds numbers and is found to be comparable to that of PAKB. Selected comparisons are made to the secondary flow results of the other PAK airfoils highlighting the endwall flow physics. The results of the complementary CFD simulations predicted a stalled blade row for a range of inlet boundary conditions set despite the well behaved profile performance documented experimentally. The limitations of the predictions are discussed.

Finally, the results and contributions of the study are summarized in Chapter 9. The conclusions of the study are drawn and recommendations for future work are also outlined. 


\section{Chapter 2}

\section{Review of Previous Work}

\subsection{Introduction}

Endwall flows in axial turbomachinery have been studied in depth for the past thirty years. This chapter gives an overview of pertinent literature with an emphasis on the relationship between loss generation and various aerodynamic and geometric changes. The chapter begins with a overview of loss generation within a turbine blade passage, followed by a detailed description of the physics of the endwall flow. The significance of blade surface and endwall boundary layers to entropy generation is discussed next, with particular emphasis on the transition to turbulence. Following that, the effect of significant aerodynamic and geometric parameters are discussed. The chapter concludes with a review of endwall contouring design techniques and a summary of experimental investigations. This chapter serves as a general overview of the open literature and relevant references are discussed in greater detail in subsequent chapters. 


\subsection{Losses in Axial Turbomachinery}

Traditionally loss correlations have used three categories to classify the loss generation mechanisms within a turbine. Empirical correlations are developed for each and the results are linearly superimposed to determine overall performance of the blade passage (Ainley and Mathieson, 1951; Dunham and Came, 1970; Kacker and Okapuu, 1982; Sharma and Butler, 1987; Benner et al., 2005a; Benner et al., 2005b). The three loss components are typically defined as:

Profile losses: losses generated in the boundary layers of the aerofoil in a uniform two-dimensional flow. The loss due to the sudden area increase at the trailing edge and the losses accumulated by mixing out of the wake are usually included in the profile loss.

Secondary losses: losses associated with the endwall boundary layers and the various vortical structures that are seen downstream of the trailing edge in the endwall region. Secondary loss structures are three-dimensional having significant components of spanwise and pitchwise velocity.

Tip leakage losses: losses occurring as a result of flow passing through the tip clearance gap of rotor blades.

Tip leakage losses occur mostly in rotors and can be reduced by minimization of tip clearances or by the use of shrouds. Tip leakage losses are not a focus of the investigation presented herein. The idealized loss breakdown given above assumes that the three loss components are independent of one another, even though experimental evidence suggests they are not. Thus, loss correlations must be used with care, and are best employed with a good physical understanding of the flow. Profile losses are closely tied to the generation of the boundary layer on the blade's surface. The development of the boundary layer and its relation to the profile loss is discussed in section 2.3.1.

Many previous studies of secondary losses in axial turbines have resulted in a good 
physical understanding of the flow behaviour. This thesis builds on the physical understanding developed by these studies. Sieverding (1985), Gregory-Smith (1997) and Langston (2001) all offer detailed descriptions of the flow in the endwall region and comprehensive assessments of research in the area. More recently, Benner (2003) has also presented a detailed literature review of the subject.

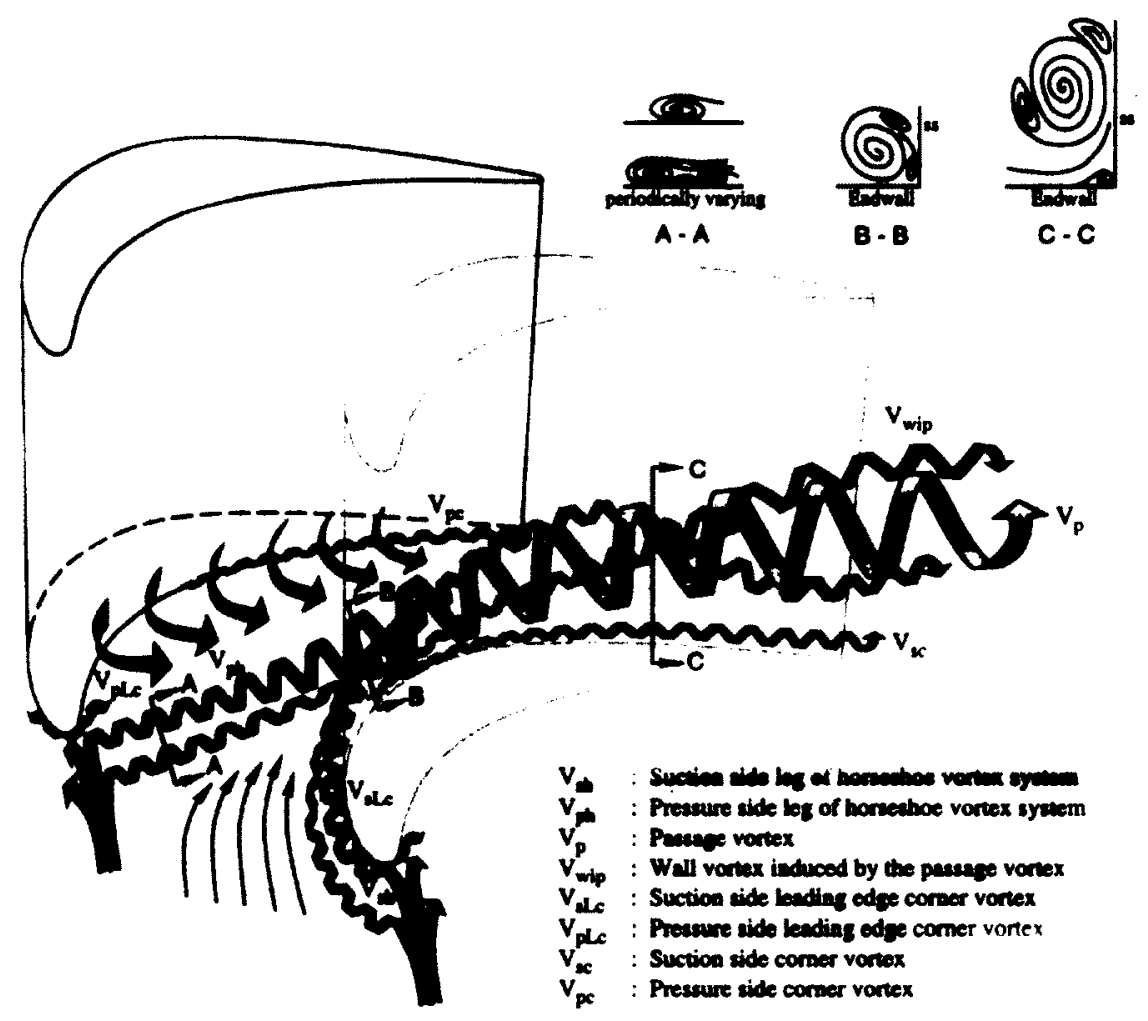

Figure 2.1: Endwall flow structures, reproduced from Wang et al. (1997)

Sjolander (1975) presented one of the first detailed descriptions of the viscous flow field in the endwall region of an annular cascade. Shortly thereafter Langston et al. (1976) used inner passage measurements to quantify losses through a planar cascade as a function of axial location. Since then many researchers have proposed interpretations of the flow structure in the endwall region. One well accepted description is that of Wang et al. (1997) shown in Figure 2.1. The interpretation proposed in Fig. 2.1 was developed using 
multiple smoke wires, a laser sheet, and a high speed camera in a linear cascade. Eight wires placed parallel to the endwall were used to generate smoke with the tunnel operating at relatively low Reynolds number of $2.7 \times 10^{4}$. The time dependent state of the vortex structure was documented by positioning the laser sheet and camera at various locations in the test section. Upstream of the leading edge (section 'A-A', Fig.2.1) a periodic multivortex pattern was observed. This is consistent with the findings of Eckerle and Awad (1991) who took non-intrusive measurements upstream of a cylinder-endwall junction using a laser doppler anemometry system. By examining velocity vector plots from the plane of symmetry upstream of the cylinder, the authors noticed a well defined periodic circulation was initiated similar to that seen by Wang et al. This recirculation has implications on heat transfer in the area, increasing the local heat transfer rate from the freestream to the endwall and rendering film cooling ineffective (Praisner and Smith, 2006).

Section 'A-A' cuts through the pressure-side leg of the horseshoe vortex that wraps itself around the leading edge of the cylinder. The suction side leg of the horseshoe vortex $\mathrm{V}_{\mathrm{sh}}$ quickly evolves into a single vortex and remains tucked into the suction-surface/endwall junction until it encounters the pressure side leg at the merging point. The pressure side leg $\mathrm{V}_{\mathrm{ph}}$ is swept across the blade passage by the cross passage pressure gradient. In doing so it seems to entrain some inlet boundary layer fluid and grows in size and strength. $V_{\mathrm{sh}}$ and $V_{\mathrm{ph}}$ have opposite senses of rotation. The pressure side leg eventually develops into what is called the passage vortex $V_{p}$. The passage vortex encounters the suction leg vortex of the adjacent passage at the merging point and lifts it up the blade surface (section 'B-B') eventually wrapping it around itself. At the endwall/suction-surface junction a new corner vortex $\mathrm{V}_{\mathrm{sc}}$ forms as a result of the viscous shear generated by the passage vortex. The suction side corner vortex also has a sense of rotation opposite that of $V_{\mathrm{ph}}$.

As $V_{p}$ grows within the passage it is lifted off the endwall along the suction surface. While doing so it interacts with the suction surface boundary layer giving rise to a small, very 
intense wall vortex $V_{\text {wip }} . V_{\text {wip }}$ has a sense of rotation opposite that of the passage vortex and experimental evidence suggests that it too is composed of inlet boundary funneled into the secondary flow structure as indicated my the arrows located between the blades. Thus at the merging point, a portion of the incoming inlet boundary layer climbs the wall becoming $\mathrm{V}_{\text {wip }}$ and a portion remains in the suction-surface/endwall corner becoming $\mathrm{V}_{\mathrm{ph}}$. At section 'C-C' the passage vortex is seen surrounded by the three other dominant vortices having a sense of rotation opposite to it. A revealing visualization of a secondary flow is that of Sieverding and Van Den Bosche (1983) who used coloured smoke emitted from a hot wire located in the inlet boundary layer of a rotor blade cascade. The leftmost blade passage of Figure 2.2 shows $V_{p h}$ being swept across the passage and becoming the core of the passage vortex. The central blade passage shows the inlet boundary layer (blue smoke) and $V_{\text {sh }}$ interacting with $\mathrm{V}_{\mathrm{ph}}$ (both shown in orange) to become the passage vortex. Unfortunately the visualizations of Sieverding and Van Den Bosche were conducted on the opposite endwall to those of Wang et al., so the sense of rotation of all the vortices are opposite making a direct comparison somewhat awkward. Because the passage vortex evolves from $V_{p h}$ and its development is driven by the cross passage pressure gradient, it only seems logical to attempt to modify the cross passage pressure gradient in hopes of reducing the strength of the secondary flow and the losses associated with it.

\subsection{Flow Physics in Axial Flow Turbines}

This section discusses the physics of the flow through an axial flow turbine blade passage and builds on the introduction above. Profile losses measured downstream of a turbine blade are strongly dependent on the state of the pressure and suction surface boundary layers. Through recent years, the suction surface boundary layers of a number of low-pressure turbine cascades have been examined to determined their likelihood of separation under 


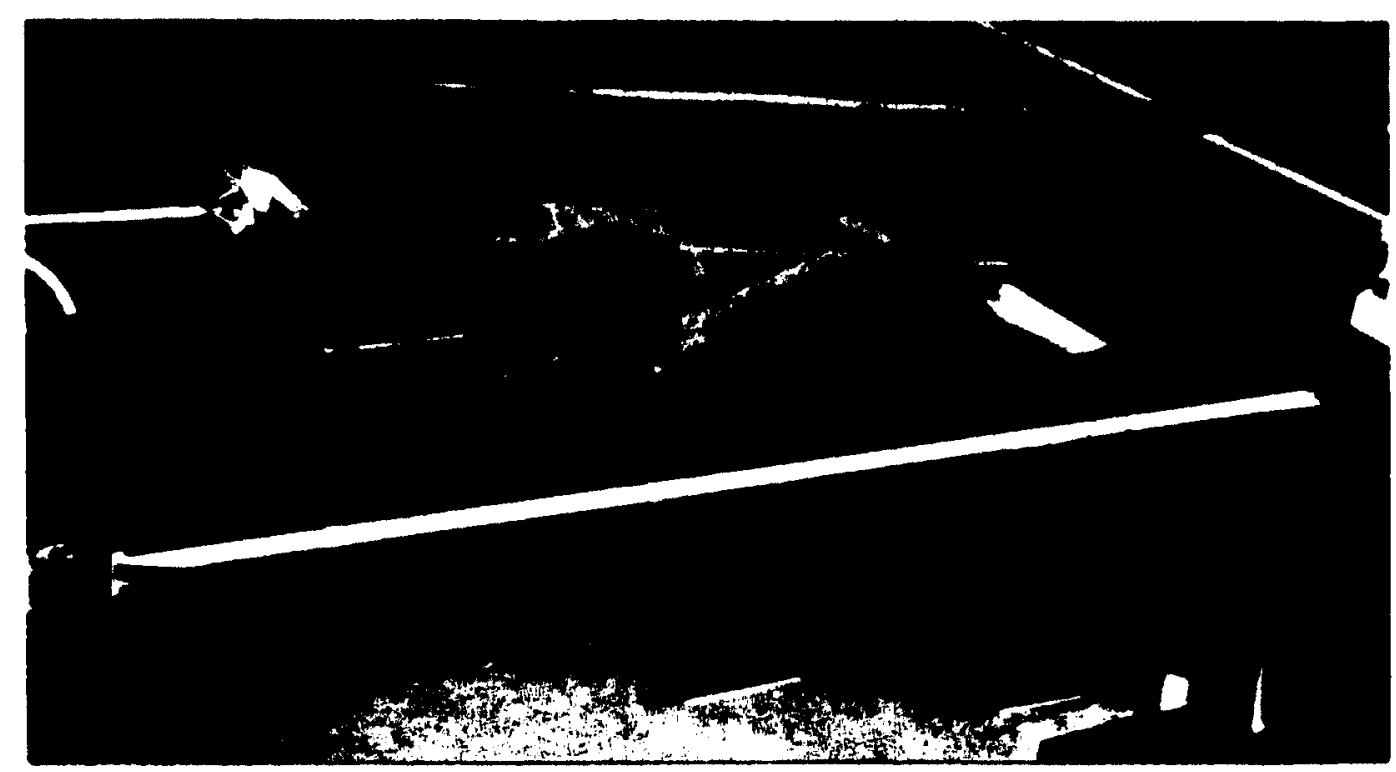

Figure 2.2: Endwall flow visualization in a rotor blade passage - smoke wire inside inlet boundary layer, reproduced from Sieverding and Van Den Bosche (1983)

adverse pressure gradient conditions. Locally, an attached boundary layer can have one of three states; laminar, transitional, or turbulent. The process of transition can occur by several modes: natural, reversed (re-laminarization), bypass, separated, or periodicunsteady $^{1}$. The prediction of boundary layer transition, separation and reattachment has been at the forefront of research with the goal of increasing blade loading and avoiding fully-separated stall. Although the transition process was not studied directly in the current work, it is important to the loss generation within the blade passage. The discussion of the transition process is followed by an overview of the interaction of secondary flow with the blade surface boundary layers. The section concludes with the effects of transition on the endwall boundary layer and the development of the secondary flow.

\footnotetext{
${ }^{1}$ Periodic-unsteady transition occurs as a result of the intermittent disturbances caused by passing wakes generated by upstream components in the engine. Although their importance is noted, periodic unsteady effects are beyond the scope of this thesis.
} 


\subsubsection{Blade Surface Boundary Layers}

The location of transition from laminar to turbulent flow is a function of the Reynolds number, streamwise pressure gradient and freestream turbulence intensity. Transition begins with the amplification of small disturbances within the boundary layer and a transfer of energy from the mean flow by an entrainment process. What happens next is dependant on the specific mode of transition.

Natural transition occurs in instances of very low freestream turbulence. The process of natural transition is discussed in detail in chapter 15 of Schlichting and Gersten (2000). Figure 2.3 (taken from White (1974)) shows the process schematically for a flat plate at zero incidence with no streamwise pressure gradient. When a critical momentum thickness Reynolds number is reached, the laminar boundary layer becomes unstable and develops oscillations that stretch in the spanwise direction. These oscillations occur at a well defined frequency and are known as Tollmien-Schlichting (T-S) waves. As the T-S waves convect downstream, they develop into three-dimensional waves and roll up into loop vortices. The loop vortices break down into a region of locally calm and locally turbulent flow. The locally turbulent regions, known as turbulent spots grow and coalesce as they are convected downstream to form a fully turbulent boundary layer.

The length of plate required to undergo transition from laminar to turbulent flow is a function of the Reynolds number and the streamwise pressure gradient. The free stream turbulence intensity (FSTI) affects the location of transition inception and also the length of the transition zone. Roberts and Yaras (2003) conducted hotwire measurements on a flat plate with three imposed pressure gradients at various Reynolds numbers and FSTIs. Their results are summarized as follows. Increasing the FSTI moves the location of turbulence inception closer to the leading edge of the plate, while decreasing the FSTI has the opposite effect. Decreasing the Reynolds number or imposing a favourable pressure gradient causes 


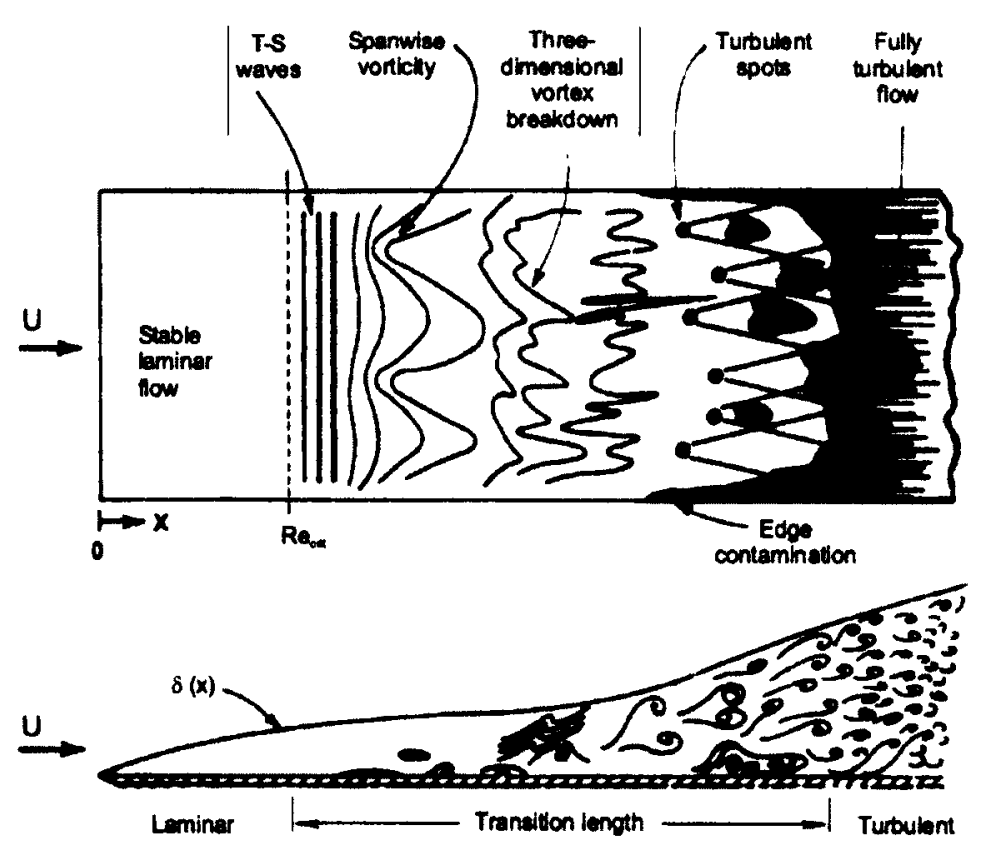

Figure 2.3: Schematic representation of natural transition, reproduced from White (1974))

transition to occur over a longer distance. In fact, if the favourable pressure gradient is strong enough, it will prevent transition from happening all together. A strong favourable pressure gradient can also cause a turbulent boundary layer to revert to a laminar one regardless of Reynolds number or FSTI through the process of reversed transition or relaminarization. Increasing the Reynolds number, imposing an adverse pressure gradient, or increasing the FSTI will tend to shorten the distance required for complete transition.

If the freestream turbulence intensity is high the mechanism of transition is altered. The boundary layer transforms from a laminar state to one that exhibits turbulent spots without showing signs of T-S waves or loop vortices. This process is referred to as bypass transition. Mayle (1991), in his 1991 IGTI Scholar lecture suggests that bypass transition is the most likely mode of transition for fully attached suction surface boundary layers in turbomachinery. 
In a strong adverse pressure gradient a laminar separation can occur before the onset of transition. Downstream of the separation point the boundary layer may then undergo separated transition in the free shear layer (presumably if the Reynolds number is low enough, transition may not occur). Depending on the flow characteristics and the length of blade available, the flow may reattach as a turbulent boundary layer forming a laminar-separation/turbulent-reattachment bubble on the surface. This process is shown schematically in Figure 2.4 (reproduced from Zhang, 2002). The top portion of Figure 2.4 shows the blade surface static pressure coefficient $\left(\mathrm{C}_{\mathrm{p}}\right)$ as a function of downstream distance $(\mathrm{x})$. The bottom portion of the figure is a schematic view of the streamlines and velocity profiles. $\mathrm{X}_{\mathrm{S}}$ marks the location of the separation, $\mathrm{X}_{\mathrm{R}}$ denotes the location of reattachment and $\mathrm{X}_{\mathrm{t}}$ and $\mathrm{X}_{\mathrm{T}}$ indicate the onset and completion of transition, respectively. Laminarturbulent transition in the free shear layer of separation bubble shares characteristics of both boundary layers and other free shear layers. Transition can be instigated by Tollmien-Schlichting waves as described above, or by the Kelvin-Helmholtz $(\mathrm{K}-\mathrm{H})$ instability occurring in the free shear layer between the recirculating flow and the freestream. When the $\mathrm{K}-\mathrm{H}$ instability dominates, transition ensues quickly after the roll-up and amalgamation of vorticity, with the time-averaged reattachment point $\left(\mathrm{X}_{\mathrm{R}}\right)$ occurring afterwards. It is important to note that a separated shear layer does not always reattach. If the adverse pressure gradient downstream of the separation point is too severe it can cause the airfoil to stall.

The reattachment of a separation bubble is a highly unsteady process. Downstream of the reattachment point large-scale vortices referred to as rollers are shed. McAuliffe and Yaras (2005) found that in some instances, these shed vortices occurred at the first subharmonic of the primary instability frequency prevailing in the separated shear layer. This implies that a vortex pairing was present (pairs of vortices are shed separated by fluid having no vorticity for a distance of twice the instability wavelength). 


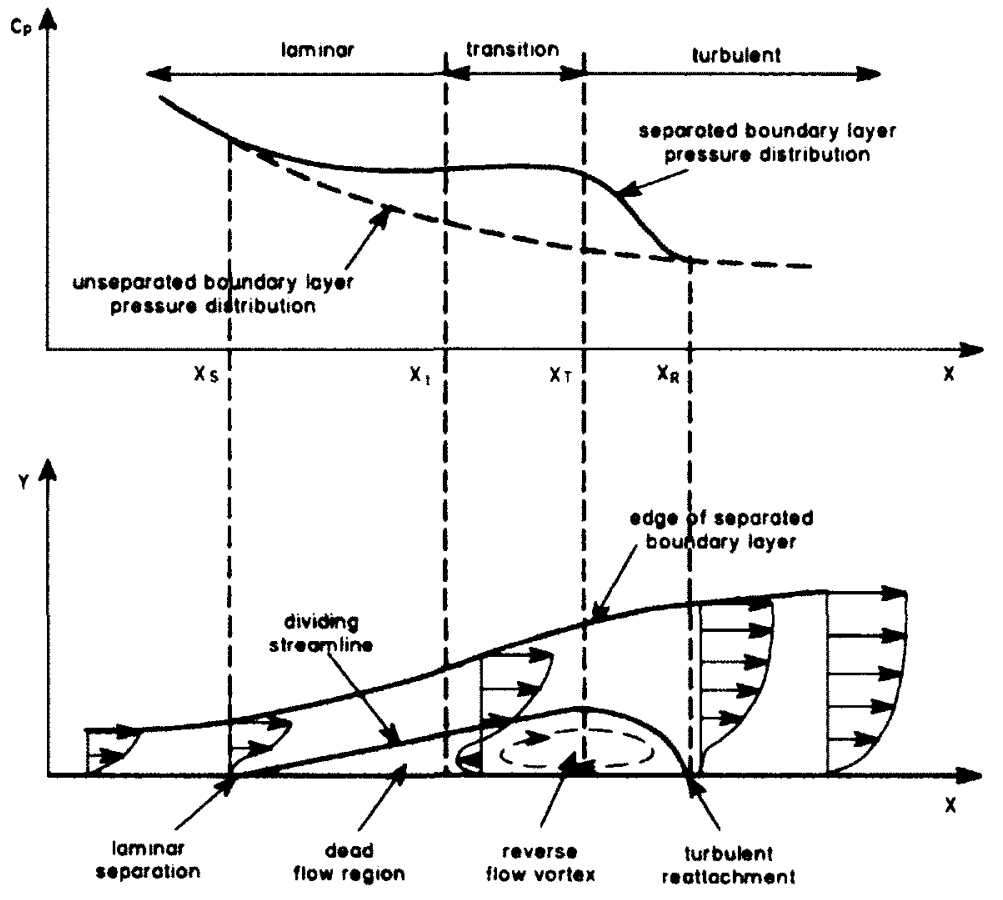

Figure 2.4: Schematic representation of a laminar-separation/turbulentreattachment bubble, reproduced from Zhang (2002)

Separation bubbles are not limited to suction surface boundary layers. Mahallati (2003) found the presence of a pressure surface separation bubble on PAKB at some of the operating conditions tested by examining the static pressure distribution around the airfoil. Brear et al. (2002a) also studied a linear cascade of engine representative low-pressure turbine blades. The authors found that a pressure surface separation contributed significantly to the profile loss, especially at negative incidence. Hotwire measurements taken within the bubble found that the transitional separated shear layer appeared to experience the KelvinHelmholtz instability.

The transition process and the presence of a separation bubble each have implications on the entropy generated within the boundary layer. These implications will be discussed further in regards to the variation of aerodynamic parameters in sections $2.5,2.6,2.7$, and 2.8 . 
For now, it is worth mentioning that entropy generation in boundary layers is a result of viscous shear work caused by gradients in velocity. For typical boundary layers, most of the entropy generation occurs near the surface where the velocity changes most rapidly (Denton, 1993). The local rate of entropy generation is also a function of the state of the boundary layer. Take for example the results of O'Donnell and Davies (2000) who took hotwire measurements normal to the suction surface of a blade at midspan in a low speed linear cascade. The cascade was a scaled version of the midspan cross-section of a high pressure turbine rotor blade. By documenting the boundary layer velocity profile, they were able to compute local entropy production rates as a function of downstream distance from the leading edge. Their results show that the entropy production rates are highest in the first $18 \%$ of the suction surface length where the boundary layer is thin and laminar. This is in accordance with Figure 2.5 reproduced from Denton (1993) which shows the dissipation coefficient as a function of the momentum thickness Reynolds number $\left(R e_{\Theta}\right)$. The dissipation coefficient is a non-dimensional parameter that quantifies the amount of entropy generated per unit of surface area. Figure 2.5 shows results for a flat plate boundary with no pressure gradient. A favorable pressure gradient tends to reduce dissipation, while an adverse pressure gradient has the opposite effect. Near the leading edge where the boundary layer begins $\operatorname{Re}_{\Theta}$ is small and the entropy production is highest.

\subsubsection{Secondary Flow and Endwall Boundary Layers}

In section 2.2 it was mentioned that secondary flows interact with the blade's suction surface boundary layer. This interaction can be seen in Figure 2.6 reproduced from Benner (2003). Figure 2.6(a) shows a clearly defined suction surface separation bubble that extends from a non-dimensional spanwise distance of 0.1-0.9. The flow separates at a non-dimensional suction surface length of 0.6 and reattaches at 0.75 . The triangular wedges marked by the separation $S_{2 S}$ show the extent of the interaction of the secondary flow with 


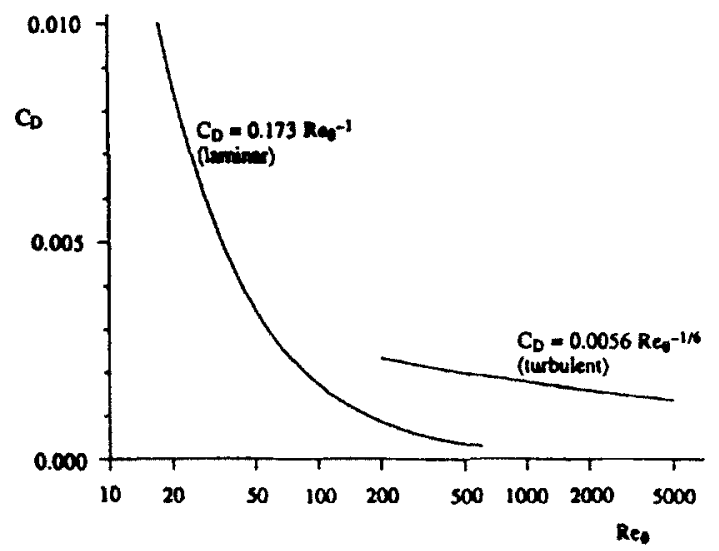

Figure 2.5: Dissipation coefficient for laminar and turbulent boundary layers, reproduced from Denton (1993)

the suction surface boundary layer and the separation bubble. Suction surface boundary layer fluid is directed between $S_{2 S}$ and the separation bubble. Near the reattachment of the bubble the oil flow visualization turns upstream showing a recirculation. This recirculation generates entropy through viscous dissipation and likely imparts low momentum fluid from within the separation bubble to the secondary flow structure.

Separations $S_{4}, S_{5}$, and reattachment $R_{4}$ result from the viscous interaction of the passage vortex with the suction surface boundary layer. Figure 2.6(b) shows an interpretation of the flow field in the endwall region proposed by Benner (2003) based on experimental results. The passage vortex induces two smaller vortices on the suction surface of the blade which are denoted SS1 and SS2. The vortices are bound by $\mathrm{S}_{4}$ and $\mathrm{S}_{5}$, and separated by $\mathrm{R}_{4}$.

One final note to make on the interaction of the secondary flow with the suction surface boundary layer relates to the penetration depth $\left(Z_{T E}\right)$. The penetration depth is the spanwise distance that the secondary flow separation $S_{4}$ penetrates along the trailing edge from the endwall. For example, Figure 2.6(a) shows that the penetration depth is about $15 \%$ of span. The concept of penetration depth was first introduced by Sharma and Butler 


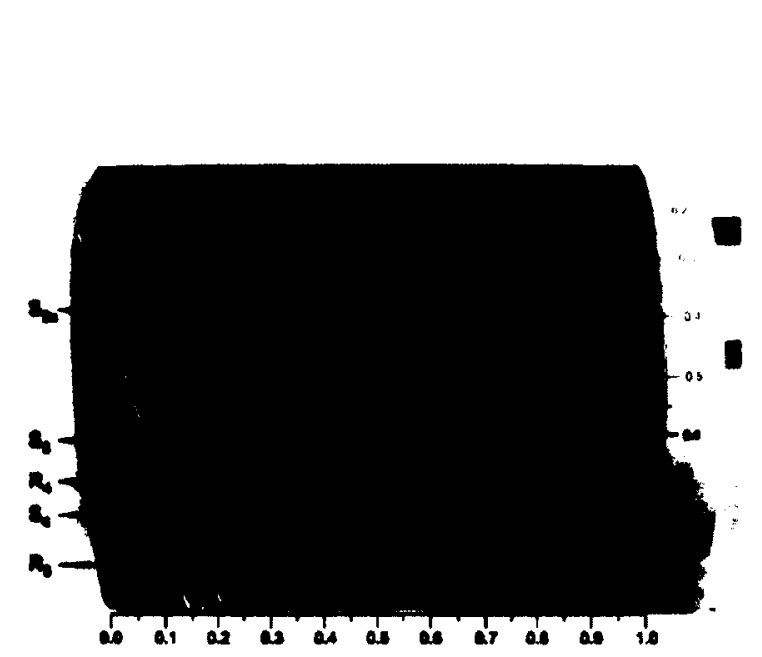

(a) Suction surface oil flow visualization

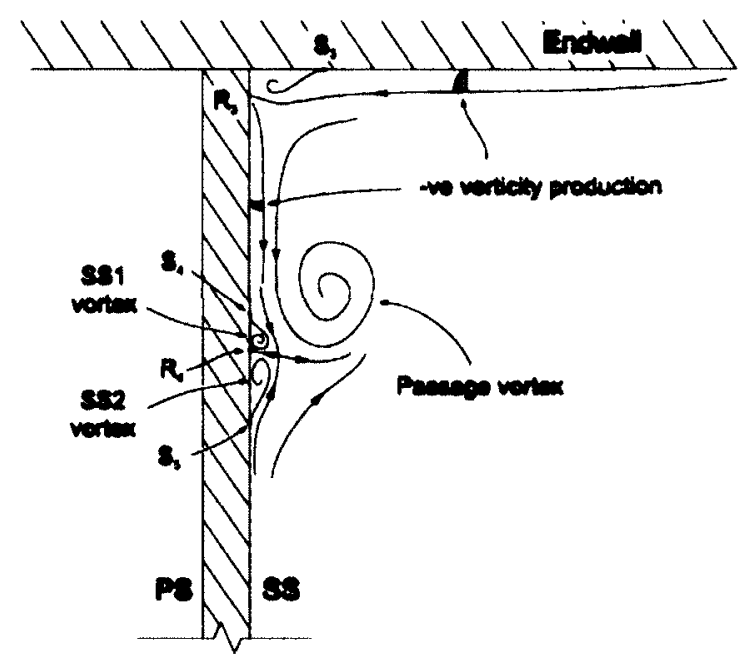

(b) Schematic representation looking upstream

Figure 2.6: Interaction of passage vortex with suction surface boundary layer, reproduced from Benner (2003)

(1987) for correlating the size of the secondary flow and in turn the losses associated with it. Benner et al. (2005a) and Benner et al. (2005b) present a new correlation for penetration depth based on more recent results.

The state of the incoming boundary layer (whether it is laminar or turbulent) has an effect an the vortices forming in the endwall region. The state of the inlet boundary layer was studied by de la Rosa Blanco et al. (2003) in a linear cascade of low-pressure turbine airfoils, who found that the position and strength of the endwall vortices were affected. The authors concluded that stagnation pressure losses generated in the blade passage were influenced by the amount of inlet boundary layer fluid entrained into the secondary flow as the passage vortex rolls up. The entrainment process varied based on the position of the inlet boundary layer roll-up, which was dependant on the state of the inlet boundary layer. The interaction of the passage vortex with the pressure-surface separation bubble was also affected by the change in position of the passage vortex.

In the endwall region, the presence of the blades causes the endwall boundary layer to 
undergo a series of separations and reattachments. As mentioned in Section 2.2, the strong adverse pressure gradient seen by the endwall boundary layer as it approaches the leading edge of the cascade causes a flow separation. This separation is seen on the righthand side of Figures 2.7 (a) and (b) reproduced from Benner (2003). The horseshoe vortex forms downstream of the $S_{2}$ separation streamline. The suction side legs of these separation lines are denoted with a subscript ' $\mathrm{S}$ ' and the pressure side legs with a subscript ' $\mathrm{P}$ '. The ' $\mathrm{S}$ ' legs of the separations quickly converge on the suction side of the blade while the ' $\mathrm{P}$ ' legs are swept across the passage toward the adjacent blade by the cross-passage pressure gradient.

Sieverding (1985) notes that the convergence of the pressure leg with the suction surface can have one of two outcomes which he calls 'strong' and 'weak'. A weak convergence results from a low angle of incidence between the passage vortex and the blade surface. In such a case, the corner vortex $\left(\mathrm{V}_{\mathrm{sc}}\right.$, Fig. 2.1) is not formed. In the case of a strong interaction, which is the case in Figure 2.7, $\mathrm{V}_{\mathrm{sc}}$ is generated and it is bound by $\mathrm{S}_{3}$ on the endwall and $R_{3}$ on the blade suction surface (Figure 2.6).

On the endwall (Figure 2.7), downstream of $R_{2}$ a new boundary layer is formed. The passage vortex convects fluid from the freestream to this region and it energizes the inner boundary layer. The extent to which this happens depends on the strength of the passage vortex. Vera et al. (2009) studied this intra-passage boundary layer in a four stage lowpressure turbine rig. Hot film sensors mounted on the endwall were used to make inferences on the state of the boundary layer. The authors concluded that the new boundary layer formed behind the pressure leg of the horseshoe vortex is transitional, and that the transition process happens in the direction of the secondary flow. The authors compared the rig data to that taken in a linear cascade and drew similar conclusions.

The incoming stagnation streamline (rightmost dashed line, Figure 2.7(b) ) meets $\mathrm{S}_{1}$ at the horseshoe vortex's saddle point indicated by $\left(A_{1}\right)$. The location, and upstream 

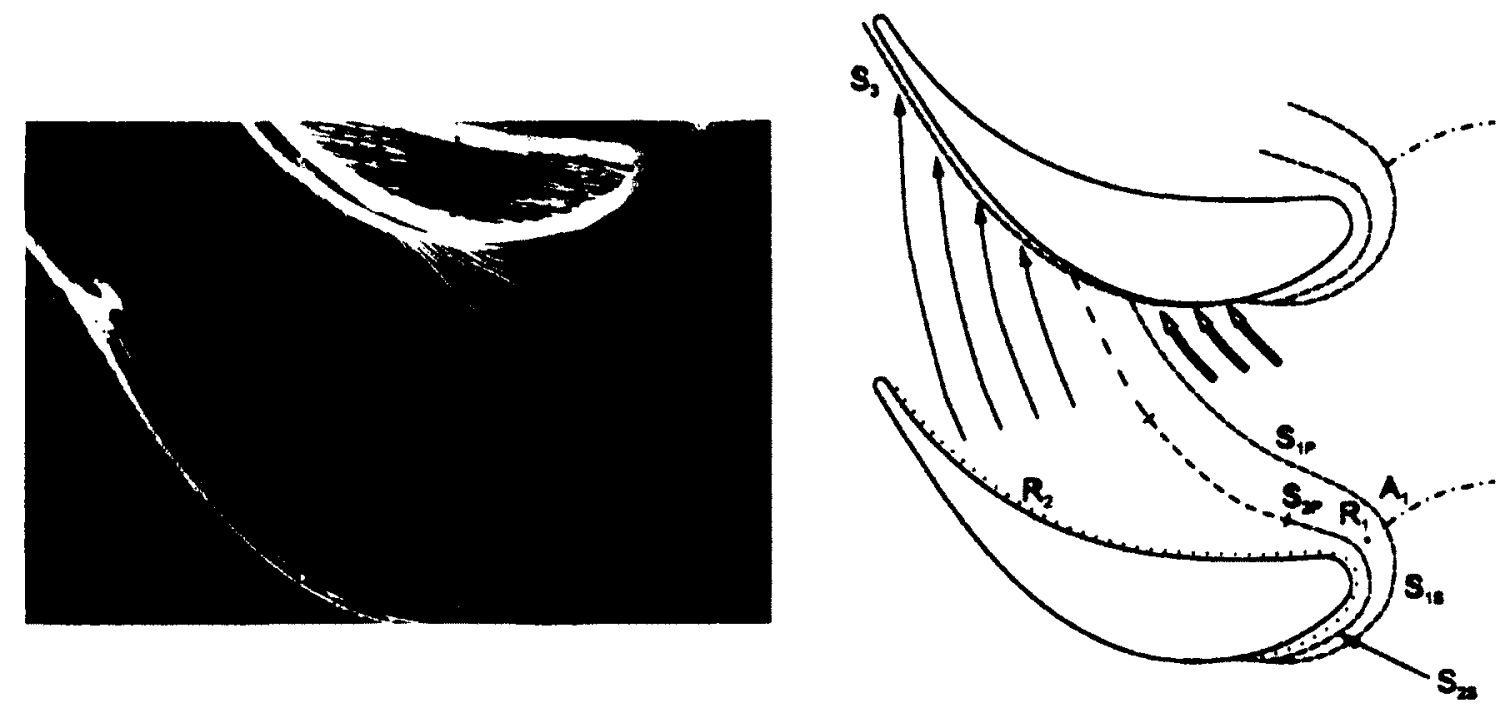

(a) Endwall surface oil flow visualization

(b) Schematic representation of endwall flow structure

Figure 2.7: Endwall boundary layer in the presence of a linear cascade, reproduced from Benner (2003)

distance of the saddle point is dependent on a number of parameters including the freestream Reynolds number; the angle of incidence of the cascade, the leading edge diameter, the overall loading level and loading distribution. One would expect that the losses generated in the endwall region were a function of the incoming boundary layer thickness, since there is more low momentum fluid to mix with the freestream in the passage vortex. However, the results of several researchers have shown this not necessarily the case. Marchal and Sieverding (1977) conducted low speed experiments in a cascade of nozzle blades at two inlet boundary layer thicknesses. Total pressure measurements (with a five-hole pressure probe) were conducted at six axial positions throughout the cascade. They found that the difference in integrated pressure loss at each axial position was nearly the same as the difference in total pressure at the inlet resulting from the change in boundary layer thickness. Gregory-Smith and Graves (1983) found similar results comparing cobra probe measurements at three different axial locations within a high-turning linear cascade. Hodson and Dominy (1987a), and Chen and Dixon (1986) also support the notion that secondary 
losses are independent of inlet boundary layer thickness. It can be noted that there is some dependence of secondary loss for very thin inlet boundary layers (Sauer and Wolf, 1994).

\subsection{Blade Loading}

Blade loading is the aerodynamic force per unit span generated by an infinitely long aerofoil of constant cross section. It is the result of the integrated static pressure over the pressure and suction surfaces of the blade profile.

In the mid 1930's Zweifel (1945) began advocating the use of a tangential force parameter for measuring the aerodynamic loading of axial turbine blades. His argument was that the commonly used lift coefficient $C_{\alpha}$ from external aerodynamics is sensitive to inlet angle and varies greatly with different velocity triangles. Zweifel's new loading parameter was defined as the actual tangential force on the blade $\left(F_{T}\right)$ over what he called the ideal tangential force $\left(F_{T_{\text {ideal }}}\right)$. Zweifel's ideal tangential force is generated if the pressure (concave) surface sees the flow's total pressure along its length and the suction (convex) surface feels the outlet static pressure. The outlet static pressure was selected since it is the lowest pressure that can be present if there is to be no adverse pressure gradients on the suction surface. If there are no pressure losses in the blade passage, $F_{T_{\text {ideal }}}$ per unit span is equal to the outlet dynamic pressure multiplied by the axial chord of the blade. For incompressible flow with constant axial velocity, Zweifel's loading parameter is defined as

$$
\mathrm{Z}_{\omega}=\frac{F_{T}}{F_{T_{\text {ideal }}}}=2 \cdot \cos ^{2} \alpha_{2} \cdot\left(\tan \alpha_{2}-\tan \alpha_{1}\right) \cdot \frac{s}{C_{x}}
$$

where $s$ is the blade spacing (blade pitch), $C_{x}$ is the axial cord and $\alpha_{2}$ and $\alpha_{1}$ are the inlet and outlet flow angles. Thus for a given cascade with a fixed pitch-to-cord ratio, the Zweifel number is only a function of the inlet and outlet flow angles. For example, if the inlet angle 
of attack was increased and the outlet flow angle remained unchanged, the Zweifel number of the blade would increase.

Using data available at the time, Zweifel deduced that the minimum profile loss through a turbine cascade occurred at around $\mathrm{Z}_{\omega}=0.8$. A value of 0.8 gave an optimum balance between friction losses caused by too many blades and suction surface separation losses that result from too few. Today's turbine blades are designed with great attention to the behaviour of the suction surface boundary layer. This has allowed blades to reach pressures well below the outlet static on the blade suction surface without experiencing detached flow separation. As a result blades with Zweifel coefficients greater than 1.0 are commonplace. For example, PAKB (the baseline cascade in this study) has a Zweifel coefficient of 1.08 . The PAKD airfoils, also examined, are considered to be highly-loaded, having a Zweifel coefficient of 1.36. For comparative purposes it should be noted that typical "high" blade loadings quoted in open literature are within the range 0.9 to 1.2 . The highest published turbine blade loading in the open literature at the time when this thesis was undertaken was the cascade of Houtermans et al. (2004) at $\mathrm{Z}_{\omega}=1.47$. Currently, the collaborative efforts of PWA and Carleton University have resulted in the highest published blade loading, with the PAKF cascade having a Zweifel coefficient of 1.78 (Praisner et al., 2008).

\subsection{Reynolds Number Effects on Loss Generation}

Blade row loss is directly related to the state of the suction surface boundary layer and how it varies with Reynolds number $(R e)$. As discussed in Section 2.3.1, at a given axial location the boundary layer can be either laminar, transitional, separated or turbulent. The state of the boundary layer is dictated by the pressure gradient history it sees up to that point as imposed by the blade surface curvature, in addition to the freestream properties such as Reynolds number or freestream turbulence intensity. 
At a very high Reynolds number the suction surface boundary layer will likely undergo turbulent separation resulting in a thick wake and relatively high loss. As the Reynolds number is decreased, separation is avoided and the boundary layer undergoes transition at some point along the suction surface. The blade profile loss will be lowest when the optimum amount of wetted area is covered by laminar flow. As the Reynolds number is reduced further, a threshold will be reached where the onset of a separation bubble occurs. The presence of a separation bubble increases both the profile and secondary loss of a blade row. These effects are discussed below.

The profile loss can be increased for several reasons. The separation bubble will increase the boundary layer thickness at the trailing edge which results in higher mixing losses downstream of the blade. The presence of a bubble also increases the rate at which entropy is produced within the boundary layer itself due to viscous friction in the recirculating fluid, its interaction with the freestream, and the mixing out of the unsteady bubble reattachment. Popovic (2005) studied the midspan behaviour of the PAKD-F aerofoil over a range of Reynolds numbers and samples of his results are shown in Figures 2.8 (a) and (b). Figure 2.8 (a) shows the blade surface static pressure distribution versus the non-dimensionalized axial chord for a freestream turbulence intensity of $1.5 \%$ and length scale $(\Lambda)$ of $14 \mathrm{~mm}$. The upper set of curves show the pressure distribution for the suction surface. A separation bubble that spans from $67 \%$ to $85 \%$ of axial chord is seen at a Reynolds number of 25,000 . At a Reynolds number of 50,000 and above Popovic suggests that the boundary layer undergoes bypass transition and avoids separation. Figure 2.8 (b) shows the variation in mixed out profile loss with Reynolds number for the same freestream turbulence conditions. As the Reynolds number decreases, the profile loss increases. From 150,000 to 50,000 a small but systematic increase in loss was found. This is the net result of two counteracting phenomena. 


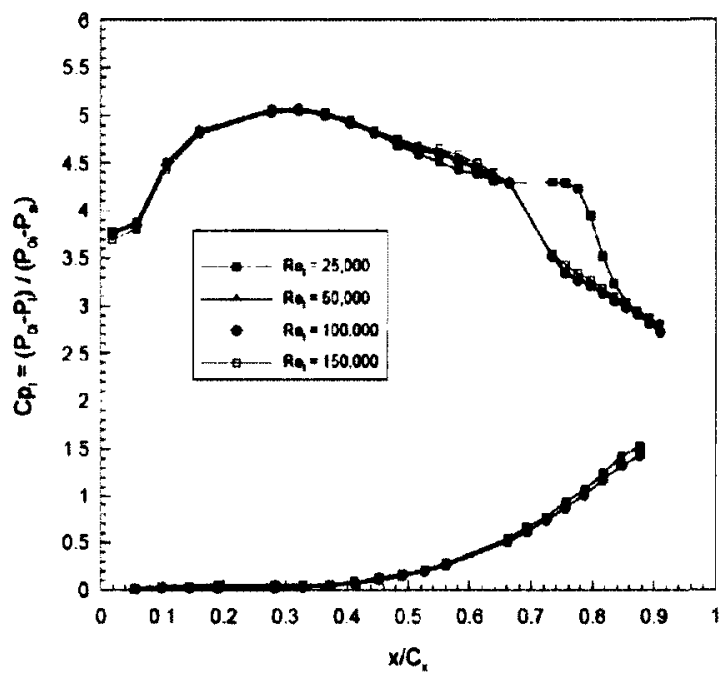

(a) Blade loading

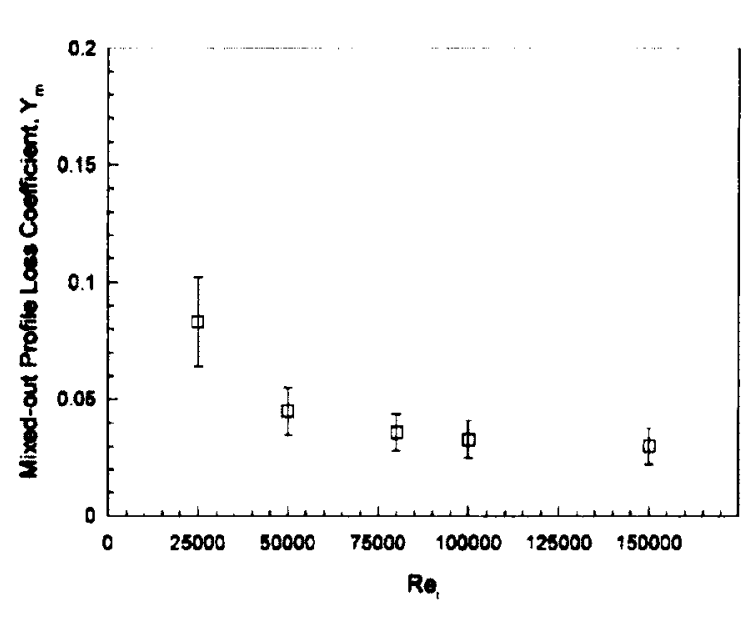

(b) Mixed-out profile loss

Figure 2.8: PakD-F data, 1.5\% FSTI, $\Lambda=14[\mathrm{~mm}]$, reproduced from Popović (2005)

- As the Reynolds number decreases the inception of transition moves downstream on the blade surface increasing the amount of wetted area covered by laminar flow. Figure $2.5^{2}$ shows that for the range of $300<R e_{\Theta}<700$ the state of the boundary layer can be either laminar or turbulent. Over this range, a laminar boundary layer produces lower losses than a turbulent one by a factor of between 2 and 5 . So a longer laminar boundary layer has the effect of decreasing loss.

- A decrease in $R e$ also reduces the local momentum thickness Reynolds number $\left(R e_{\Theta}\right)$ across the entire suction surface length. Referring to Figure 2.5, the laminar portion of the boundary layer near the leading edge is very sensitive to reductions in $R_{e} e_{\Theta}$, thus having a tendency to increase loss.

For this particular airfoil, level of freestream turbulence and length scale, it appears that the second phenomenon dominates as the Reynolds number is reduced. At a Reynolds number of 25,000 a sizable jump in profile loss is seen as a result of the presence of the separation bubble.

The presence of the separation bubble also increases the loss associated with the

\footnotetext{
${ }^{2}$ Figure 2.5 presents results for a boundary layer with no streamwise pressure gradient, however the trends are similar when a pressure gradient exists. The effects of favourable and adverse pressure gradients on the dissipation coefficient were outlined in Section 2.3.1 where the figure was introduced.
} 
interaction of the secondary flow with the suction surface boundary layer. For example Hodson and Dominy (1987a) studied a linear cascade in a closed loop transonic wind tunnel capable of varying the Reynolds number independently of the Mach number. The cascade was designed to have an isentropic exit Mach number of 0.71 and an isentropic exit Reynolds number of $2.9 \times 10^{5}$. Holding the exit Mach number constant the effects of Reynolds number were investigated. At twice the design $R e$ the secondary losses were reduced by roughly $3 \%$ from the design conditions, at half the design $R e$ the secondary losses increased by $31 \%$. The authors attributed the increase in secondary loss to the sizeable separation bubble that grows in size on the suction surface of the aerofoil as the Reynolds number is reduced to $R e=1.5 \times 10^{5}$. Figure 2.9, reproduced from Hodson and Dominy (1987a) shows oil surface flow visualizations of the suction surface at both off design Reynolds numbers. No separation is present at $R e=6.0 \times 10^{5}$ (Fig. 2.9 (b)), however, Figure 2.9 (a) has a large separation bubble with an apparent recirculation where it comes into contact with the secondary flow. The authors suggest that the strength of the interaction with the secondary flow would be increased by the lower static pressure in the separation bubble. Also, the unsteadiness and mixing that would accompany a large separation would add to loss generation.

Reynolds number was not varied in the present test matrix, however, the experiments were conducted at an elevated Reynolds number relative to the PAK airfoil design condition $\left(R e=8.0 \times 10^{4}\right)$. Tests were conducted at $R e=1.26 \times 10^{5}$ in an attempt to reduce the influence of blade surface separation bubbles on secondary loss generation.

\subsection{Effect of Free Stream Turbulence Intensity on Loss Gen- eration}

Free stream turbulence intensity (FSTI) was not varied in the present study, however it is reviewed here because it is important to the mechanisms of loss generation. The effect of 


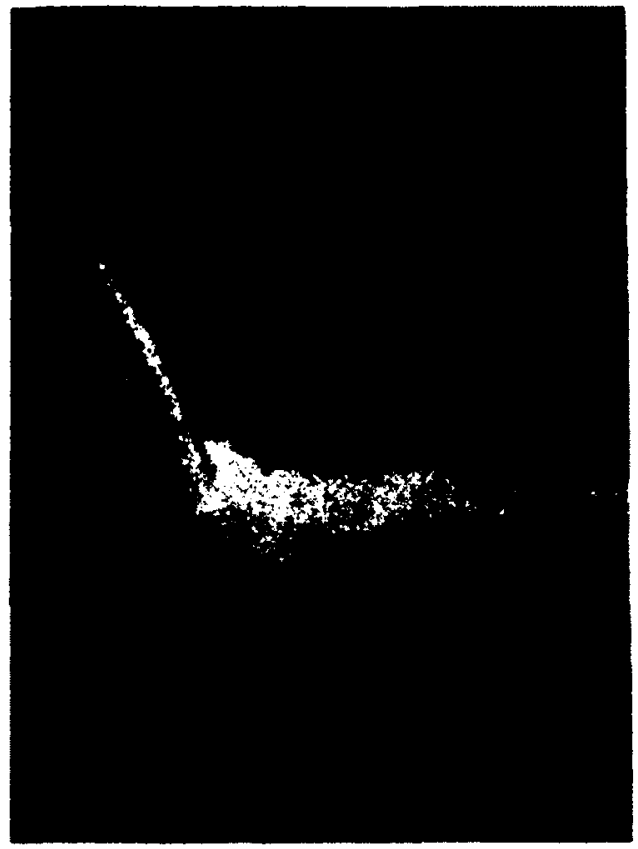

(a) $R e_{2 s}=1.5 \times 10^{5}$

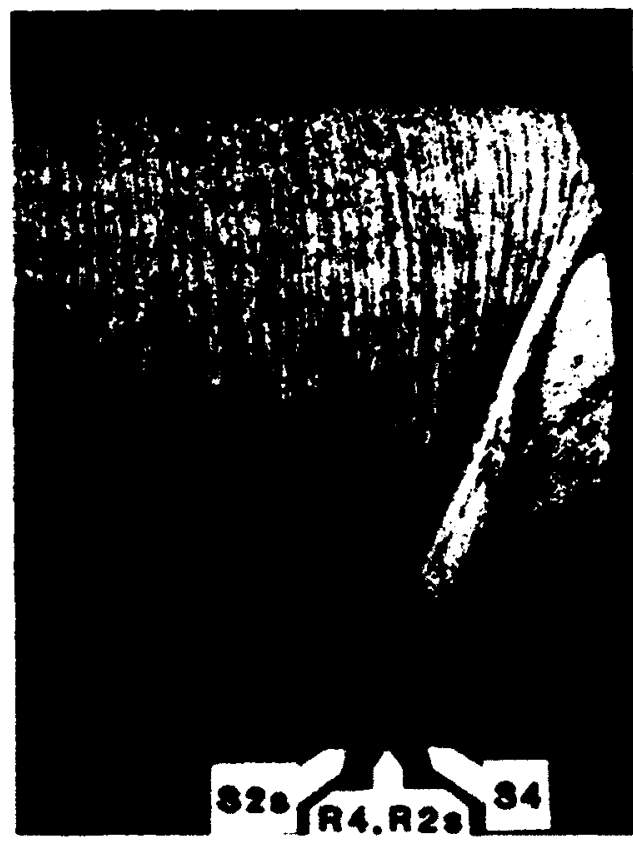

(b) $R e_{2 s}=6.0 \times 10^{5}$

Figure 2.9: Suction surface oil flow visualization, reproduced from Hodson \& Dominy (1987a)

FSTI on loss generation is similar to that of Reynolds number in that it affects the state of the blade boundary layers.

For example, if a blade experiences a laminar separation and is fully stalled at a given Reynolds number, a higher level of FSTI may cause transition to occur in a shorter distance in the free shear layer, thus allowing the bubble to reattach. A turbulent boundary layer is more resistant to separation, thus if the transition process is completed upstream of where laminar separation would have occurred, due to higher FSTI, separation may be avoided all together.

Another effect of freestream turbulence intensity can be seen on laminar-separation/turbulentreattachment boundary layers such as that of PAKD-F at a Reynolds number of 25,000 (Figure 2.8). Here a higher level of FSTI would hasten the transition process by amplifying the instabilities in the separated free shear layer. This could cause the turbulent 
reattachment to occur in a shorter distance and would result in a smaller separated region. A smaller separation bubble would produce less entropy and so the profile losses would be reduced.

The FSTI affects secondary losses mainly through the interaction of the secondary flow with the suction surface separation. Reducing the size of the separation bubble reduces its interaction of the secondary flow and thus the losses. Determining the effects of FSTI on secondary flows experimentally is often difficult because the use of turbulence generating grids modifies the endwall boundary layer. Gregory-Smith and Cleak (1992) conducted an investigation on the effects of FSTI on secondary flows. They documented a reduction in inlet boundary layer thickness, displacement thickness and momentum thickness that resulted from the installation of the turbulence generating grid and found an approximate $22 \%$ reduction in secondary loss. Zorić (2006) used a similar approach when investigating the secondary losses of the Pratt and Whitney designed PAKB and PAKD aerofoils. The turbulence generating grid resulted in a thinner inlet boundary layer and lead to lower secondary losses. In Section 2.3.2 evidence that showed that the loss in the inlet boundary layer was simply convected through the cascade was discussed. Thus it can be argued that the findings of Gregory-Smith and Cleak (1992), and Zoric (2006) show that increasing FSTI reduces secondary losses. To the present author's knowledge there is no experimental data that decouples the effect of FSTI and inlet boundary layer changes.

\subsection{Incidence Effects}

Although incidence was not varied in the present study, it may be worthwhile to examine its effect on the flow control concepts investigated in the future. The effects of incidence on blade row performance have been investigated by several researchers, for example Yamamoto and Nouse (1988), Perdichizzi and Dossena (1993), Hodson and Dominy (1987a), Benner 
et al. (2004a), Dossena et al. (2004) and Zorić (2006). The sign convention for blade incidence is such that with positive incidence, the blade row performs more flow turning (i.e. the blade loading increases at positive incidence). This is similar to the sign convention used in external aerodynamics where a wing at positive incidence produces more lift than one at zero incidence. The aforementioned studies found that a blade row at positive incidence generates higher profile and secondary losses.

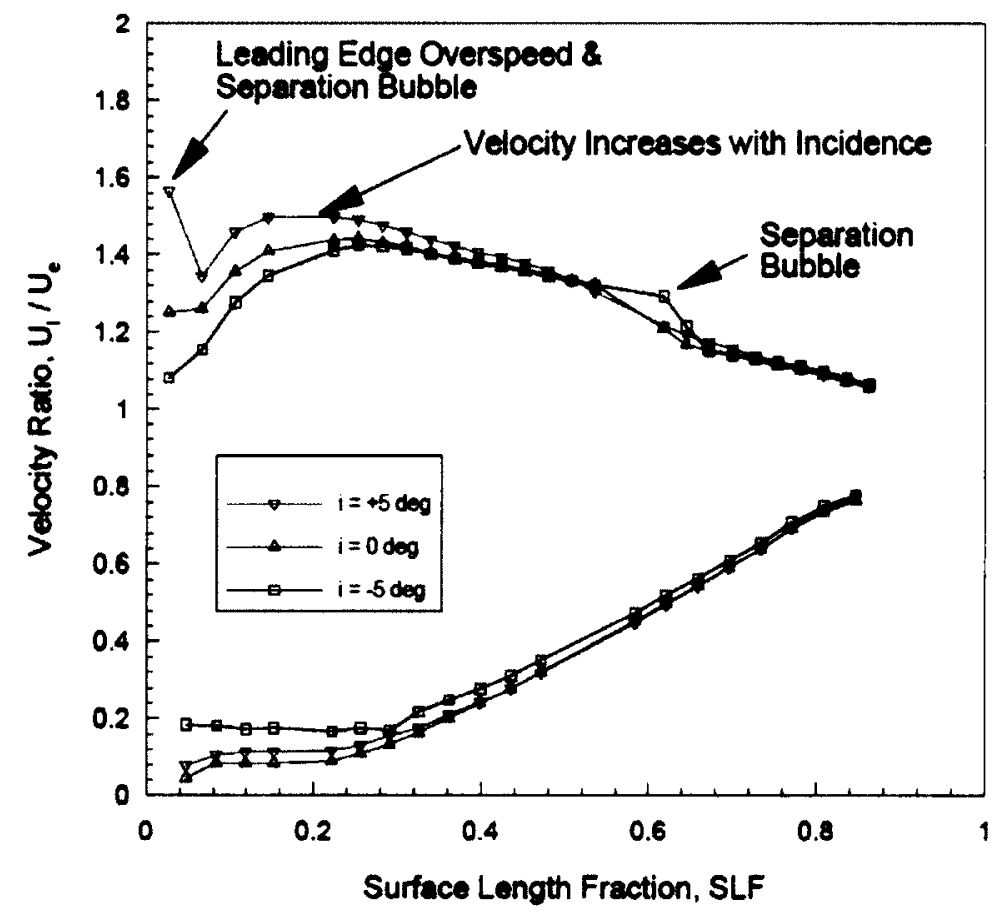

Figure 2.10: Main contributors to loss production on the suction surface, reproduced from Popović (2005)

Profile losses increase at positive incidence because the increased loading results in a higher velocity at the edge of the boundary layer. Denton (1993) showed that entropy production in boundary layers varies with the cube of the boundary layer edge velocity. Figure 2.10 shows the velocity ratio (local velocity over mass-averaged exit midspan velocity) versus surface length fraction for the forward loaded PAKD-F at $+5,0$ and -5 degrees of incidence. A leading edge overspeed and significantly higher local velocities on the suction 
surface cause the airfoil to generate $33 \%$ higher profile losses at +5 degrees of incidence than it does at its design incidence.

It is often observed that a slight negative incidence tends to reduce profile losses with respect to the design incidence case. A small negative incidence causes the blade loading to decrease and therefore reduces the velocity at the edge of the suction surface boundary layer. For the case of PAKD-F, Figure 2.10 shows that the airfoil develops a separation bubble at -5 degrees of incidence. The appearance of the separation bubble occurs because the lower suction surface boundary layer edge velocity has the tendency to delay transition. The loss associated with the separation bubble to some degree offsets the loss reduction of the lower boundary layer edge velocity and the net result is a negligible reduction in profile loss at -5 degrees of incidence with respect to design.

At large negative incidence the profile loss are commonly higher than at design incidence. Goobie (1989), Tremblay (1990), and Hodson and Dominy (1987a) present experimental evidence that shows the existence of a loss "bucket", where losses are lowest near the design incidence. Large suction surface separation bubbles, fully separated blade stall and pressure surface boundary layer separations can all contribute to higher losses at large negative incidences.

The change in blade loading with incidence also affects the secondary losses of a cascade. At positive incidence the loading is increased over the forward portion of the suction surface length (eg, see Figure 2.10). This results in a stronger pressure gradient from the suction surface of a blade to the pressure surface of the adjacent blade. It is commonly accepted that a stronger cross passage pressure gradient in the forward section of the blade passage is responsible for generating stronger secondary flows and higher secondary losses. This will be discussed further in the following subsection. 


\subsection{Effect of Loading Distribution on Loss Generation}

Several researchers have investigated the influence of loading distribution on loss generation. The most pertinent investigations to this thesis are those of Popovic (2005) and Zorić (2006) who studied the midspan and secondary loss performance of the PAKB, PAKD-F and PAKD-A cascades. The loading distributions of all three aerofoils is shown in Figure 2.11 at design incidence with 1.5\% FSTI and inlet Reynolds number of 80,000. PAKD-F has its suction peak at almost $x / C_{x}=0.32$ while PAKD-A has its suction peak at $x / C_{x}=0.55$.

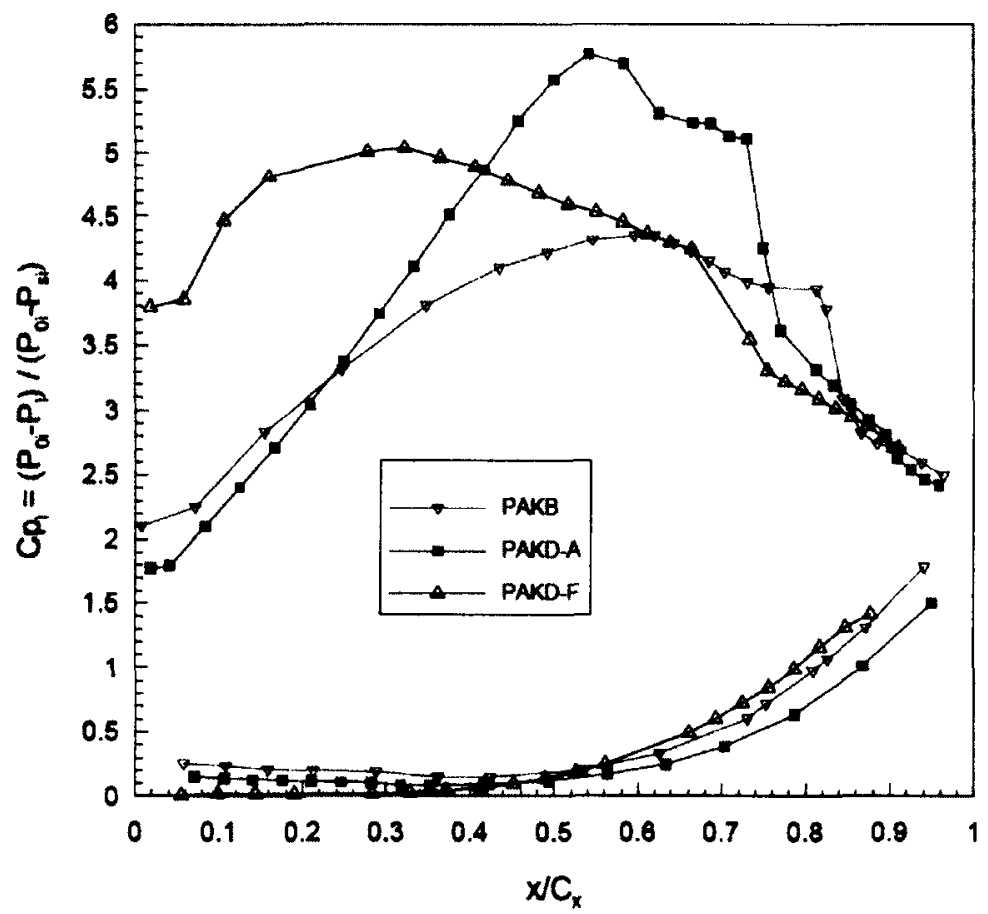

Figure 2.11: Comparison of blade loadings at design incidence $\left(\operatorname{Re}=8.0 \times 10^{4}\right)$, reproduced from Popovic (2005)

In their investigations into the effects of incidence, FSTI and Reynolds number, Popović (2005) and Zorić (2006) found that PAKD-F had superior midspan performance compared with PAKD-A but also had higher secondary losses. PAKD-A had secondary losses 
comparable to those of the more lightly loaded PAKB. However, PAKD-A had higher profile losses than PAKD-F and stalled at low Reynolds number or positive incidence. These results are agreement the study of Weiss and Fottner (1995) who conducted high speed experiments on the front loaded T104 and aft loaded T106 aerofoils. Both the studies of Zoric (2006) and Weiss and Fottner (1995) found that the front loaded aerofoils exhibited a stronger passage vortex and higher secondary kinetic energy, thus accounting for the higher secondary losses. Weiss and Fottner explained their results by the following: the suction peak near the leading edge of the T104 imposes a strong cross passage pressure gradient on the relatively thick undisturbed boundary layer entering the cascade. In the case of the aft loaded T106, the peak pressure gradient occurs near the throat where it reacts on a thin new endwall boundary layer.

\subsection{Controlling Losses Generated by Secondary Flows}

Many researchers have developed methods for controlling secondary flows and reducing the losses associated with them. Some examples of successful flow control methods that are pertinent to this dissertation are discussed below. The section begins with a review of methods not employed in the current research program. The latter part of the section focuses on flow control techniques investigated here, namely non-axisymmetric endwall contouring and a modified loading distribution.

\subsubsection{Endwall Fences}

Prümper (1972) reported that installing boundary layer fences on the hub and casing walls in turbomachinery may reduce losses. More recently, Chung and Simon (1991), and Chung and Simon (1993) have implemented a fence on the endwall near midpassage to prevent the migration of the pressure leg of the horseshoe vortex and the endwall boundary 
layer towards the suction side of blade passage. The fence had a triangular cross-section and a height of $4.5 \%$ of chord. They found that the passage vortex was weakened and decreased in size after being deflected by the fence to remain on the pressure-side of the passage. The secondary losses were reduced.

Denton (1993) argues that a mixing process undergoing an acceleration has damped velocity gradients that lead to reduced dissipation (and thereby lower mixing loss). Conversely a mixing process undergoing a deceleration should have amplified velocity gradients and increased dissipation. The passage vortex is comprised of low momentum boundary layer fluid mixing with freestream fluid in a vortical structure (Wang et al., 1997), thus forcing the vortex to remain near the pressure surface, where it experiences an accelerating favourable pressure gradient reduces dissipation.

As a followup to the work of Chung and Simon, Aunapu et al. (2000) used endwall jets to modify the endwall flow. The airflow requirements were similar to those used for film cooling. The jets were located along the same path as the fence, and were successful in deffecting the migration of the passage vortex. This work showed that endwall cooling flow could be introduced into the blade passage to favourably modify the endwall flow as well as to obtain cooling.

\subsubsection{Leading Edge Modifications}

Some authors have modified the leading edge region of the endwall to influence the formation of the horseshoe vortex. Sauer et al. (2001) tested four leading edge bulb designs applied to the T106 airfoil in a linear cascade. They found that the bulb increased the strength of the suction-side leg of the horseshoe vortex $\left(V_{s h}\right)$, which counter-rotates relative to the passage vortex. The stronger $V_{\text {sh }}$ deforms the passage vortex and delays the migration toward the suction side of the blade passage, thereby reducing loss by almost $50 \%$. 
Additionally, the authors claimed that "excellent qualitative and acceptable quantitative results were achieved" when the experimental data were compared to numerical predictions.

In a similar fashion, Zess and Thole (2002) applied a leading edge fillet to a turbine vane. The authors ran simulations on multiple fillet geometries and found a fllet $1 \delta$ high and $2 \delta$ long was effective at eliminating the horseshoe vortex. The fillet had a linear slope from the endwall to the stagnation line and was symmetric about it. The complementary experimental study used a two-component laser Doppler velocimetry system, so no loss results were obtained.

\subsubsection{Axisymmetric Endwall Contouring}

Another form of flow control can be achieved by contouring the endwall of the blade passage. Perhaps the simplest form of endwall contouring is axisymmetric, where the span of the blade passage is reduced by protruding the endwall into the flow. This form of contouring was first suggested by Russian engineers and was coined the "Russian Kink" by Warner and Tran (1987) referring to the original work of Deich et al. (1960). This form of contouring has become predominant in nozzle guide vanes, as discussed by Dossena et al. (1999). Figure 2.12 is reproduced from their paper and shows the blade and meridional profile used. The authors found that the blade row loss was reduced by $35 \%$ and the secondary loss was reduced by $55 \%$ with the application of the contoured endwall. It should be noted that the study was conducted at a high subsonic Mach number, and that if the blade row had been choked (as is normally the case for a first stage nozzle), the change in throat area would reduce the mass flow through the cascade. Some other examples of axisymmetric contouring studies include Kopper et al. (1981), Burd and Simon (2000) and Piggush and Simon (2005). In general, axisymmetric contouring is thought to work on the principle of "turn then accelerate". The endwall flow is turned in the early part of the 
passage, then accelerated as the endwalls converge. The loss is reduced since the mixing of the low momentum boundary layer fluid with the freestream occurs in a strong favourable pressure gradient (as discussed by Denton, 1993).

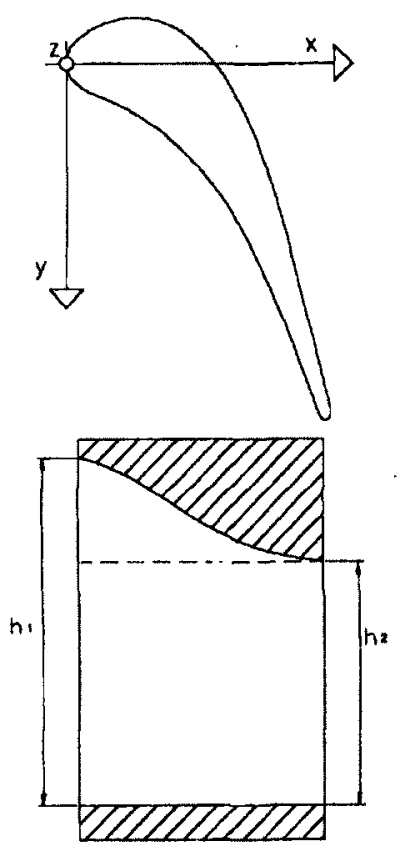

Figure 2.12: Axisymmetric endwall geometry, reproduced from Dossena et al. (1999)

\subsubsection{Non-Axisymmetric Endwall Contouring}

A different approach to controlling the endwall flow was developed by Rose (1994), and later by Harvey et al. (2000). The approach was experimentally validated by Hartland et al. (2000). Similarly to the endwall fences, non-axisymmetric endwall contouring has the objective of limiting the migration of the pressure-side leg of the horseshoe vortex across the blade passage. Rather then creating a physical barrier, the contouring locally alters the cross-passage pressure gradient so that the vortex is not drawn towards the suction surface of the adjacent airfoil to the same extent as if the endwall were planar. The local 
static pressure on the endwall is altered by means of streamline curvature. Rose (1994) explained the basic principles in terms of convex and concave wall curvature. A concave curvature causes a relative diffusion by raising the local static pressure. A convex curvature locally accelerates the flow thus reducing the local static pressure. These two principles are used in combination across the blade passage to modify the cross-passage pressure gradient. The contoured endwall of Harvey et al. (2000) is shown in Figure 2.13. Positive values of contour height represent a convex curvature (the contouring protrudes into the passage), while negative values of contour height represent concave curvature.

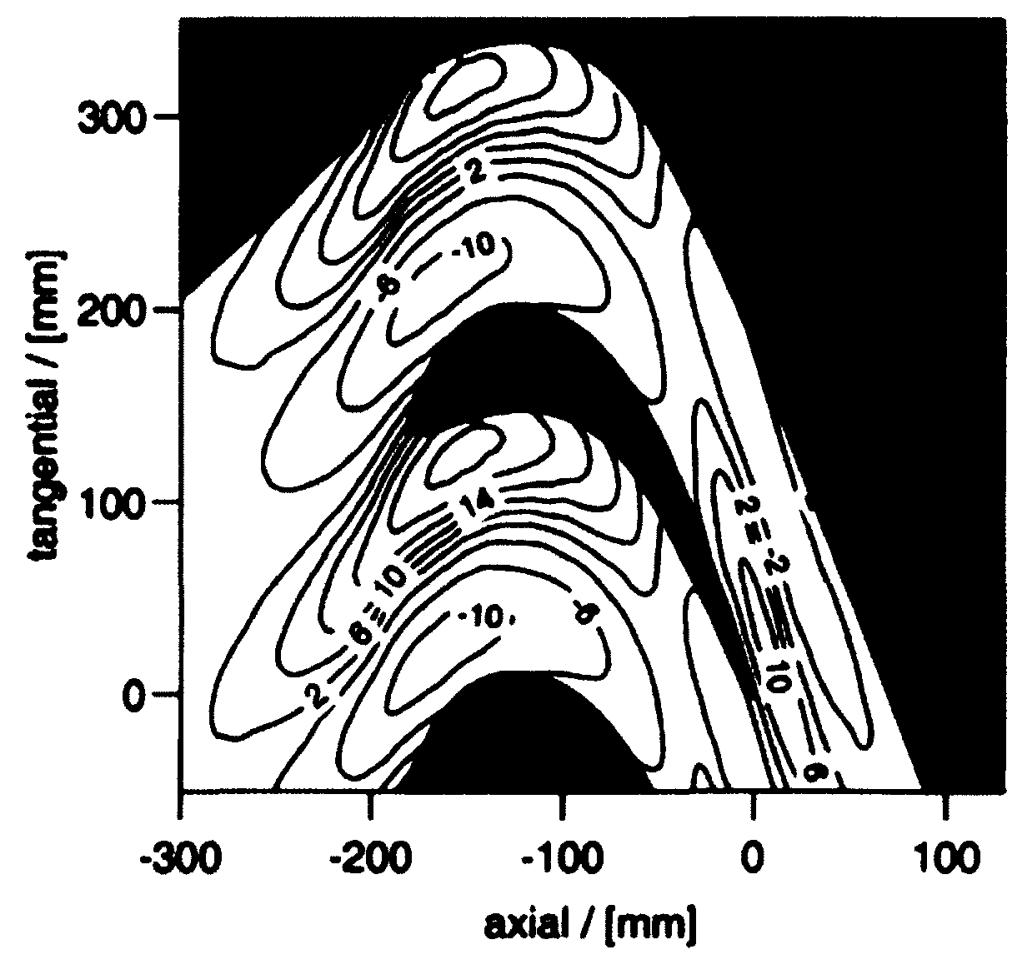

Figure 2.13: Profiled endwall geometry, reproduced from Harvey et al. (2000)

\subsubsection{Design Methodology}

One can imagine that a stronger local pitchwise pressure gradient would require a higher convex peak and lower concave valley to be neutralized. The maximum perturbations of the 
endwall would also occur in the vicinity of the peak local pitchwise pressure gradient. Thus the shape of the endwall contouring is specific to the loading (magnitude and distribution) of the airfoil and a design tool is required to optimize the configuration in order to minimize losses.

The contouring topology of Figure 2.13 was created by means of a three-dimensional linear design system. The approach was an extension of the work of Shahpar et al. (1999) who developed a forward and inverse three-dimensional linear design system for turbomachinery airfoils. Their approach is briefly described as follows:

1. Generate a set of systematic perturbations to the aerofoil geometry. The basic geometric design parameters for a blade passage are skew angle, inlet and exit angle, camber distribution, thickness, axial chord, and positional offsets in the axial and circumferential directions.

2. Compute a viscous flow field solution for all these perturbations.

3. Construct a linear sensitivity matrix using numerical differentiation.

4. Use the theory of linear superposition to to construct new geometries and flow fields.

5. Apply inverse design through matrix inversion and quasi-Newton techniques, to generate airfoil shapes that satisfy the chosen design criteria. (Minimizing secondary flow deviation or loss).

Unfortunately a contoured endwall cannot be defined by such convenient geometric parameters as those outlined above for airfoils; a new approach was needed. It was mentioned previously that the endwall static pressure distribution is a function of streamline curvature. A series of control points can be used to perturb the height of the endwall and consequently modify the flow field. The perturbation of the control points and their effect on the chosen design parameter can be used as the inputs to the direct gradients of step 3 above. The method of Harvey et al. (2000) makes use of a B-spline fit through 6 control points at 6 axial locations ( 36 perturbations per endwall). A number of design parameters 
can be selected for the optimization criteria. Harvey et al. (2000) suggest the use of the flow overturning that results from the presence of the secondary flow.

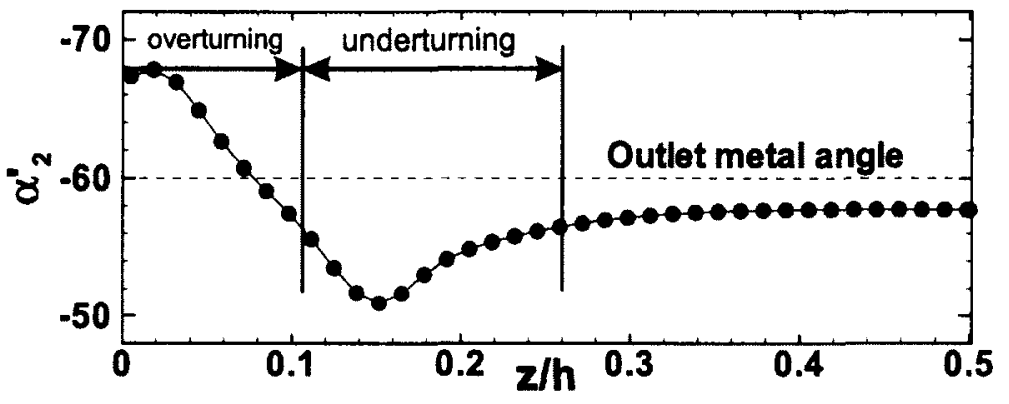

Figure 2.14: Pitchwise-mass-averaged exit flow angle for PAKB (inlet Reynolds number of 126,000 and FSTI $=4 \%$ )

An example of flow overturning is shown in Figure 2.14 for PAKB as measured with a seven hole pressure probe $40 \%$ axial chord downstream of a linear cascade by the present author. Figure 2.14 presents the pitchwise-mass-averaged exit flow angle as a function of non-dimensional distance from the endwall. The endwall is located at $\mathrm{z} / \mathrm{h}=0$ and $\mathrm{z} / \mathrm{h}=$ 0.5 is midspan. PAKB has an outlet metal angle of $-60^{\circ}$ and the aerofoil has approximately $2^{\circ}$ of deviation at midspan. The presence of the secondary flow causes $9^{\circ}$ of underturning at $\mathrm{z} / \mathrm{h}=0.15$ and $8^{\circ}$ of overturning at $\mathrm{z} / \mathrm{h}=0.02$. This exit flow angle pattern is typical of the flow field downstream of a linear cascade (a similar pattern exists downstream of an annular blade passage). Harvey et al. (2000) agree that the under/overturning caused by the secondary flow has performance implications of subsequent blade rows, placing a significant portion of the downstream blade at off design incidence. Thus, minimizing the secondary flow deviations would improve the inlet flow uniformity of the next blade row. Hodson and Dominy (1987a) also show a connection between the magnitude of maximum underturning and peak total pressure loss developed by the secondary flow. Therefore, reducing the underturning should decrease secondary loss.

The endwall contouring that is implemented in this thesis was designed by Pratt and 
Whitney Aircraft (PWA) with the use of their in-house CFD software. The details of the design are outlined by Praisner et al. (2007). Their approach is similar to that used by Harvey et al. (2000) but novel in several respects. Twenty-five control points are used to define the wall height. Five control points are situated on each of five camber lines that span one blade pitch. The central control point camber line coincides with the actual camber line of the airfoil. The outer camber lines are located at midpitch to either side of the airfoil and the intermediate cambers lines are positioned at $25 \%$ pitch to either side. The control points are aligned axially with the two outer rows of points in line with the leading and trailing edge of the cascade. The inner points are distributed evenly at axial positions of $25 \%, 50 \%$, and $75 \%$ of axial chord. The outer control points that are aligned with the leading and trailing edges are fixed at the nominal endwall height, as such, the contouring does not extend upstream and downstream of the cascade as in the case of Figure 2.13.

The remaining fifteen points are perturbed individually and a solution to each perturbation is computed using the converged solution for the planar endwall as the initial solution for the perturbed domain. The optimization technique uses a sequential quadratic programming gradient-based algorithm with mass-averaged total pressure loss in the exit plane as the objective function. Gradient searches were conducted for approximately 1000 design iterations, preformed with a RANS code using the $k-\omega$ turbulence model. Further details are presented by Praisner et al. (2007) who found trend accurate predictions of secondary loss reduction in comparison to experimental results.

\subsubsection{Experimental Validations}

The work of Harvey et al. (2000) and Hartland et al. (2000) applied non-axisymmetric endwall contouring to the linear cascade test rig at Durham University, which had been used previously for the study of secondary flows. The experimental validations of Hartland 
et al. (2000) found that blade row loss was reduced by over $15 \%$ with the application of endwall profiling. As a followup to these findings, Brennan et al. (2003) examined the potential of non-axisymmetric endwall profiling in an engine representative environment. A cold-flow, $75 \%$ scale model of the Trent 500 high-pressure turbine was used for the study. The authors describe the design of the contouring for the hub and tip walls of the nozzle guide vane and first stage rotor. The complementary experimental study is documented by Rose et al. (2001) who found that the stage efficiency could be improved by $0.4 \%$ with the use of endwall contouring. In general, these studies found that secondary losses were reduced along with deviations in the exit whirl angle (pitchwise flow angle), and secondary kinetic energy.

Ingram et al. (2005) used the same design approach as Hartland et al. (2000) to develop a contoured endwall that was supposed to push the limits of loss reduction by further altering the local cross-passage pressure gradients. This was achieved through increasing the amplitude of the endwall displacements compared with those shown in Figure 2.13. Surprisingly, the experimental measurements showed that the new profiled endwall produced higher secondary losses than the planar case, despite reducing the secondary kinetic energy by $57 \%$. Oil surface flow visualizations on the endwall showed an apparent separation of the boundary layer near the suction surface toward the inlet of the cascade that was not predicted in the design computations. The simulations treated the boundary layers as fully turbulent using wall functions and an algebraic mixing length turbulence model. Although these computations are by no means state of the art, they are typical of what is used in industry for design purposes. These findings demonstrate two key matters. First they highlight the importance of experimental validation and second they stress that the link between secondary loss and secondary kinetic energy may not be as straightforward as once thought. These two points are the primary justification for the work conducted as part of this thesis, as outlined in Section 1.3. 
Gregory-Smith et al. (2008) has combined the concepts of profiled endwalls, blade lean and leading edge extensions to minimize the secondary flow in the Durham cascade. The authors suggest that the three concepts have the following impacts on the flow. First, the endwall profiling reduces the cross-passage pressure gradient in the early part of the passage. Secondly, reverse compound lean reduces midspan losses and forces the secondary flow closer to the endwall by inducing spanwise pressure gradients. Third, the leading edge extension results in local blade sweep at the endwall further reducing the cross passage pressure gradient in the early part of the passage by reducing the blade loading there. Because of the complexity of the flow field, the authors recommend that an effective three-dimensional CFD design system must be validated with experimental testing.

\subsubsection{Other Applications}

Although endwall contouring was originally developed for use in turbines, its application is not limited to them. Harvey (2008) presents computational and experimental results for non-axisymmetric profiling applied to a linear compressor cascade at Cambridge University. The physics of the flow within the blade passage differs in some respects to what is found in turbines, as one might expect. The contouring can serve to increase the cross-passage pressure gradient, which directs higher energy fluid into the suction-surface/endwall junction mitigating the corner stall and reducing loss. Similar to what is found in turbines, the variation of outlet whirl angle can also been reduced. In a complementary study, Harvey and Offord (2008) discuss computational results for a multi-stage high-pressure compressor where profiling is applied from the first stage stator through to the sixth stage rotor. Comparable with the linear cascade results, the suction side leg of the horseshoe vortex is encouraged to migrate off the end wall and onto the suction surface of the aerofoil. In doing so it delays the corner stall that would otherwise result if this low momentum fluid remained on the endwall. 
The use of endwall profiling is not limited strictly to turbomachinery. Naylor et al. (2008) demonstrate its effectiveness for reducing the losses in annular S-shaped inter-compressor ducting. Endwall profiling was applied to the inner wall of the annulus and found to suppress the strut-hub corner separation. These results demonstrate the ability to either increase the thickness-to-chord ratio of the strut to allow for greater service access, or to increase the aggressiveness of the duct by increasing the change in radius relative to axial distance.

\subsubsection{Pressure Surface Modification}

The modified loading approach for secondary loss reduction, as introduced in Section 1.2 , has not been documented in the open literature to this author's knowledge. However, a similar technique has been implemented to suppress a large pressure-side separation by Brear et al. (2002a). In a followup paper, Brear et al. (2002b) examined a family of airfoils having the same suction surface geometry and different pressure surface profiles. Although the intent was to study the effect of the pressure surface separation, the change in secondary flow physics was analogous to that of the modified loading approach taken in this thesis. The results of Brear et al. (2002b) are therefore briefly discussed next.

Figure 2.15 shows the four pressure surface geometries investigated. Profile $\mathrm{A}$ is the original design investigated by Brear et al. (2002a). Blade D has a thickened pressure surface which was designed to completely suppress the pressure surface separation bubble at midspan. Blades $\mathrm{B}$ and $\mathrm{C}$ are three-dimensional designs having the same profile as $\mathrm{A}$ at midspan.

The experiment showed Blade D offers a $10.2 \%$ reduction in mass-averaged stagnation pressure loss relative to blade A while producing the same profile loss. The loss reduction was localized to the first $20 \%$ of span where it was accompanied by $2^{\circ}$ less peak under turning. In general the measurements showed that the secondary loss structure was reduced 


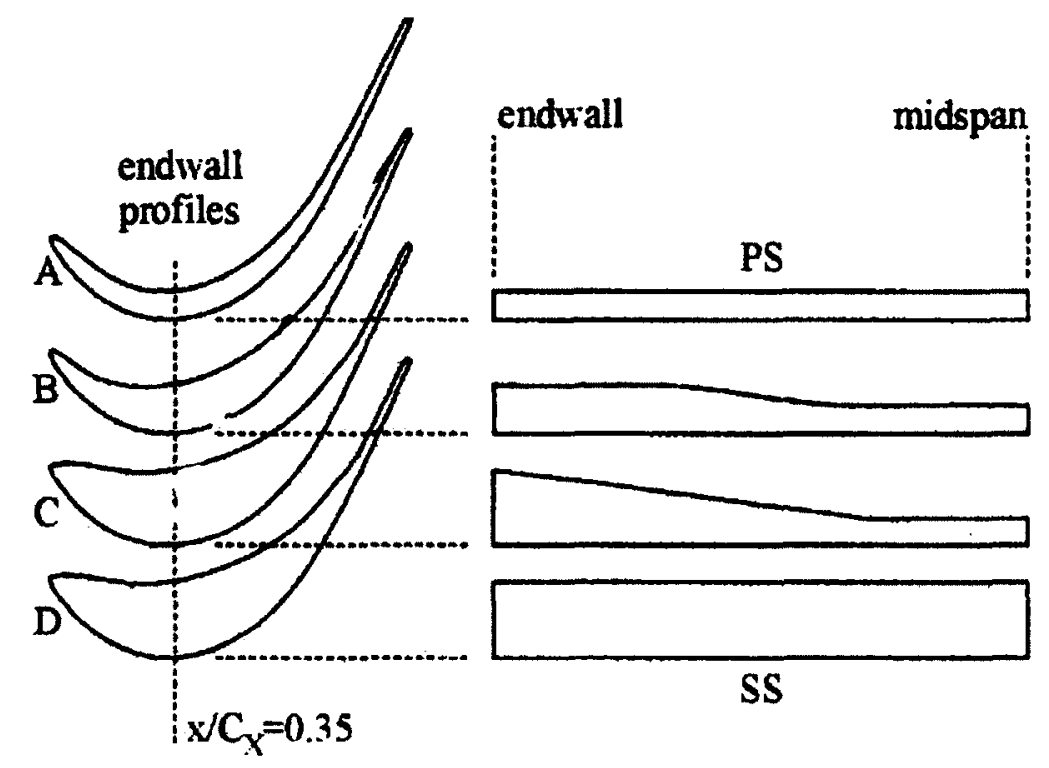

Figure 2.15: Pressure surface modifications investigated by Brear et al. (2002b)

in size and moved closer to the endwall.

The authors suggest that the lack of pressure surface separation for blade $D$ is the primary mechanism for the loss reduction, as there is less low-momentum fluid to feed the secondary flow from the pressure side of the passage. However, the results of Chapter 7 will show that similar performance benefits can be achieved by modifying the pressure surface of the airfoil when the baseline case is free of a pressure surface separation.

From the studies discussed in latter part of this chapter, it can be concluded that threedimensional modification of the static pressure field is becoming an indispensable design tool for improving component efficiency. It is also obvious that experimental validation is a vital element of the design process. In the examples cited, a firm understanding of the flow physics has led to developments in the field. 


\section{Chapter 3}

\section{Experimental Methods and Data Reduction Procedures}

\subsection{Introduction}

The experimental measurements were taken in Carleton University's Low-Speed Turbomachinery Laboratory (LSTL) using a variable incidence linear cascade test section. The wind tunnel, test section, cascade nomenclature, and cascades are discussed. The experimental instrumentation is outlined along with the probe calibration apparatus. The pressure probes are introduced, and their uncertainty is examined. The chapter concludes with a summary of cascade testing procedures.

\subsection{Low-Speed Wind Tunnel}

An open circuit wind tunnel, shown in Figure 3.1 is used as an air supply for the linear cascade test section. Air enters the wind tunnel via a bellmouth inlet drawn by a radial 
blower. The blower (Daltec Industries, Model number IE17P) is driven by an $40 \mathrm{HP}$ AC motor controlled with a Mitsubishi variable frequency drive. The flow leaving the blower passes through a honeycomb conduit to remove any swirl present, and expands through a wide-angle diffuser having a divergence angle of 19 degrees. Five evenly spaced screens within the diffuser are used to suppress boundary layer separation and produce a uniform flow to the inlet of the settling chamber.
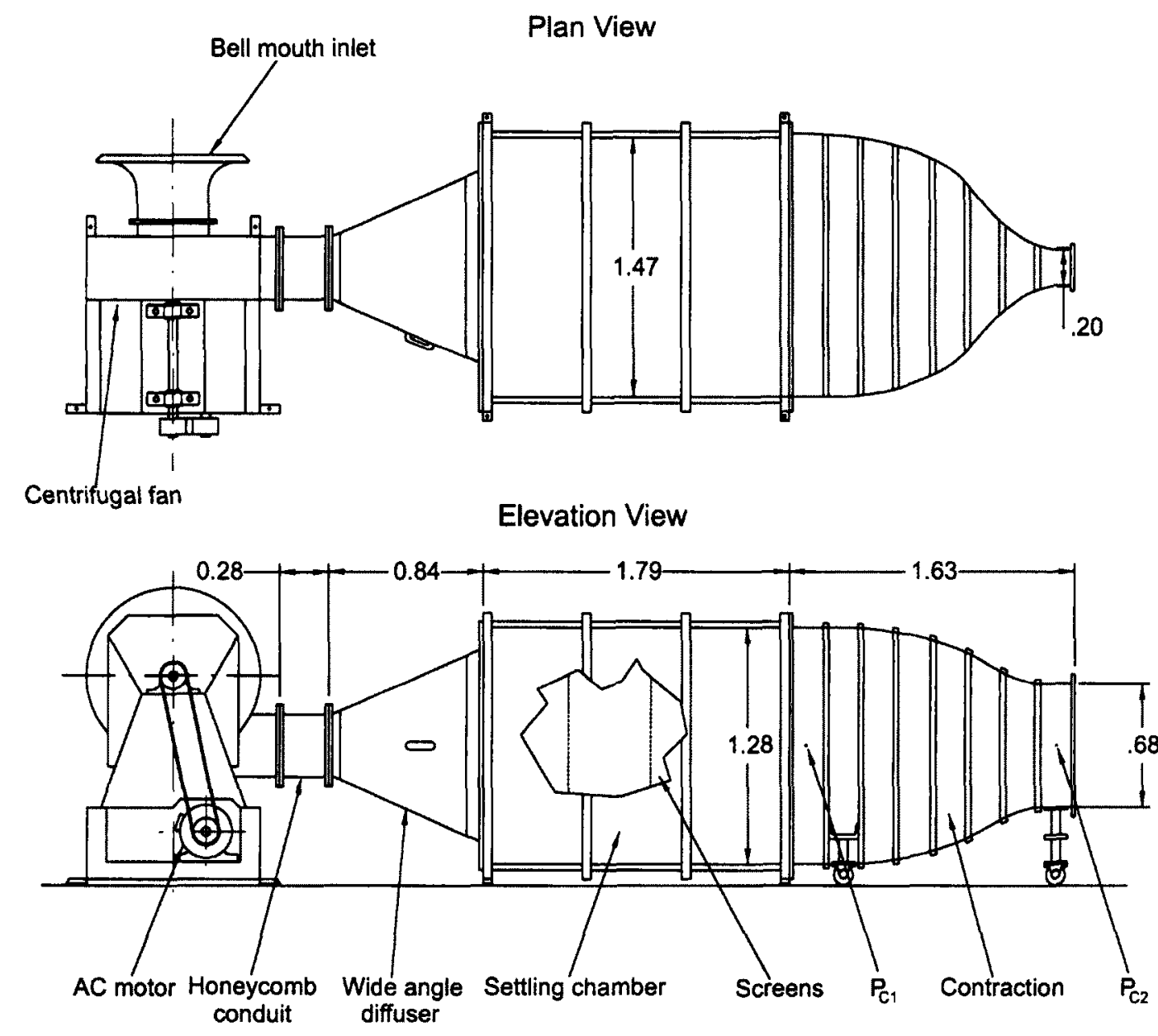

All dimensions in metres

Figure 3.1: Wind-tunnel schematic, reproduced from Benner (2003)

The settling chamber contains four additional screens to ensure uniformity and that blower blade passing frequencies are damped out. The nearly stagnant flow in the settling 
chamber is then accelerated through a square to rectangular contraction having an area ratio of 14:1. The static pressure upstream $\left(P_{C 1}\right)$ and downstream $\left(P_{C 2}\right)$ of the contraction is measured using static pressure wall tappings. The contraction pressure difference $\left(P_{C 1}-\right.$ $\left.P_{C 2}\right)$ is used to set the wind tunnel operating point and to normalize the non-dimensional coefficients. $\left(P_{C 2}\right)$ is used for the reference pressure for the differential transducers. The contraction pressure difference is sampled at the same frequency and for the same duration as the pressure probe being used in the test section. The pressure probes are described in Section 3.9. Details regarding the wind tunnel beyond what is specified here are given by Rodger (1992), who was responsible for its commissioning.

\subsection{Test Section}

The variable incidence test section, shown in Figure 3.2 was designed and commissioned by Goobie (1989). A turbulence generating grid is mounted at the inlet of the test section to produce an engine representative level of freestream turbulence intensity and turbulence length scale. Downstream of the grid, boundary layer bleed slots are used to thin the boundary layer entering the cascade.

The upstream flaps and downstream tailboards are used to set inlet flow uniformity to the cascade and outlet periodicity. The aerodynamic effects of the two are coupled via the area formed between the top flap and top tailboard, so the initial setup is an iterative process. A bypass mechanism exists below the floating wall which can be used to increase the mass flow through the blower for a given operating condition. The blockage mechanism at the upper wall improves the effectiveness of the upper flap and improves pitchwise inlet uniformity (Mahallati, 2003).

The secondary flow measurements and flow visualizations were conducted on the 
turntable wall of the test section. The opposite wall of the test section is movable and made of Plexiglas to allow visual access. For the planar endwall measurements, the blades are mounted to a rectangular metal insert. When the test section was switched to the profiled endwall configuration, the planar endwall insert was replaced with a contoured endplate. This test section has been used to study secondary flows in linear turbine cascades by Benner (2003) and Zorić (2006) thus the operating procedures and experimental repeatability are well established. The experimental uncertainty is discussed in detail in Section 3.8.

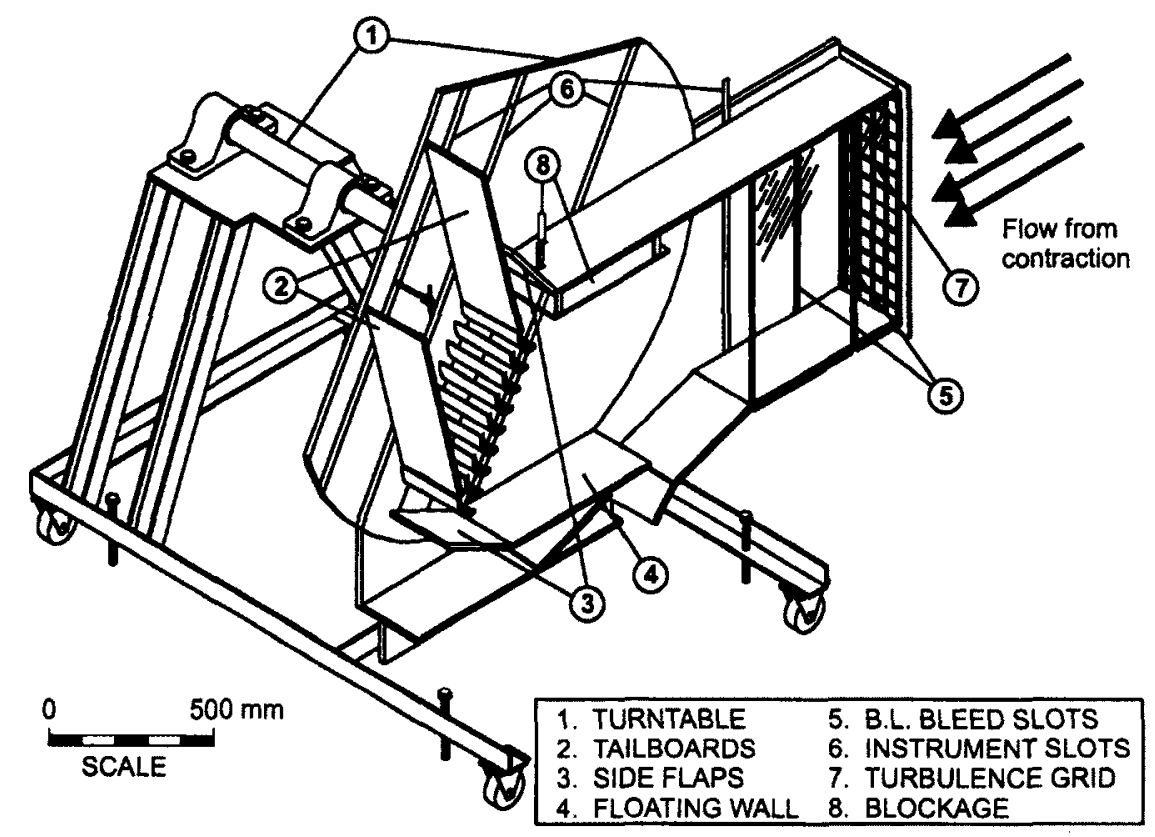

Figure 3.2: Variable incidence test section

\subsection{Research Cascades}

Figure 3.3 and Table 3.1 summarize the cascade nomenclature. The incidence of the cascade, as discussed in Section 2.7 is defined as:

$$
i=\alpha_{1}-\alpha_{1, d e s}
$$


such that positive incidence results in more lift being generated. (The cascade is shown at negative incidence in Figure 3.3). All data presented in the subsequent result chapters were taken at zero incidence. At the exit of the cascade the flow deviation angle $(\delta)$ is defined as

$$
\delta=\alpha_{2}-\alpha_{2, \text { des }}
$$

Positive deviation denotes an underturning of the flow relative to design intentions. Figure 3.3 also shows the relative location of the inlet and outlet measurement planes that are used to quantify the loss generated in turning the flow.

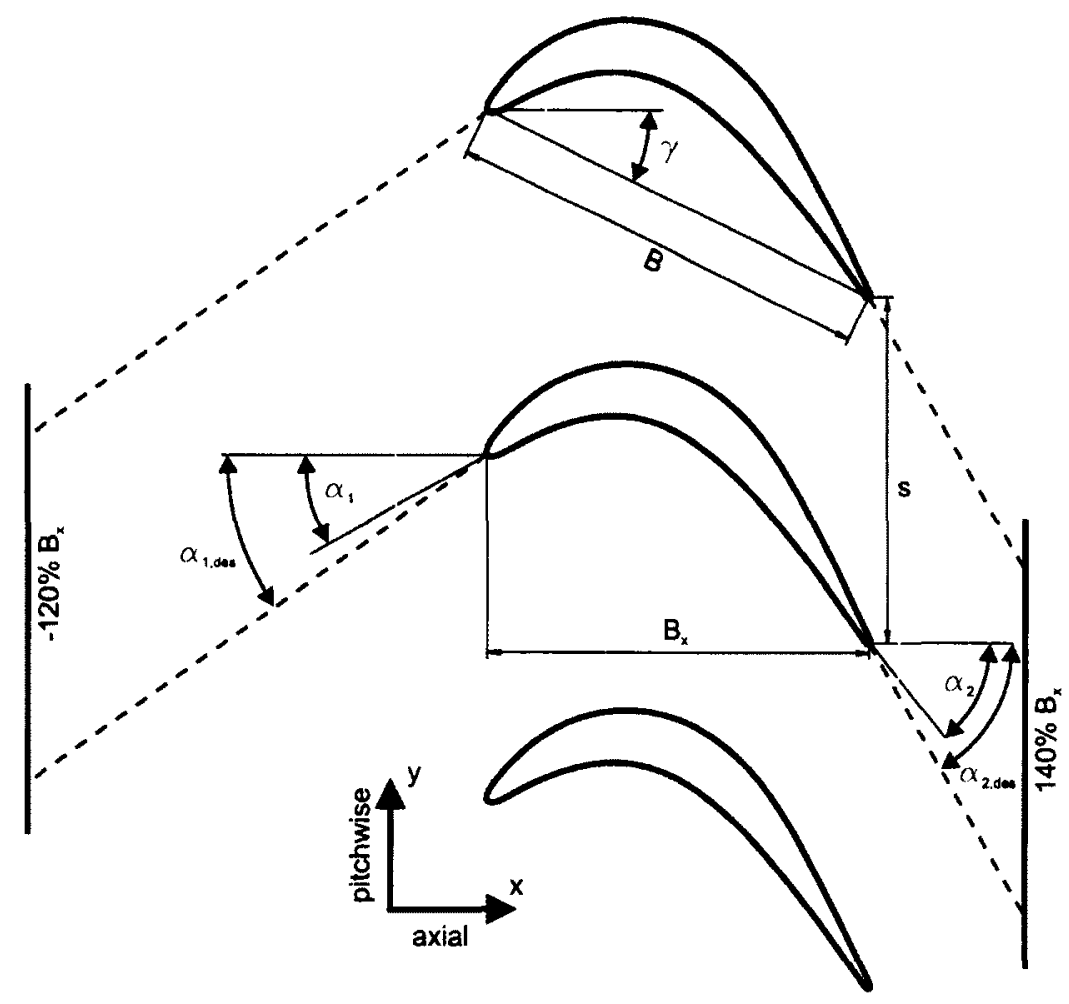

Figure 3.3: Cascade nomenclature

As outlined in Section 1.2, the test matrix examines a family of airfoils having the same design inlet and outlet velocity triangles. The geometric details of the airfoils are given in Table 3.2. Since the airfoils have the same design inlet and outlet flow angles, and nominally 
Table 3.1: Cascade nomenclature

\begin{tabular}{llll}
\hline \hline$\alpha_{1}$ & actual inlet flow angle & $\alpha_{1, \text { des }}$ & design inlet flow angle \\
$\alpha_{2}$ & actual outlet flow angle & $\alpha_{2, \text { des }}$ & design outlet flow angle \\
$\mathrm{B}$ & true chord & $\mathrm{B}_{\mathbf{x}}$ & axial chord \\
$\mathrm{s}$ & pitch spacing & $\gamma$ & stagger angle \\
\hline
\end{tabular}

the same axial chord, variations in Zweifel coefficient result from changes in pitch spacing (See Eq. 2.1). The airfoil cross-sections and corresponding Zweifel coefficients are presented in Figure 3.4.

Table 3.2: Cascade geometry

\begin{tabular}{lccccc} 
& PAKB & PAKD-A & PAKD-F & PAKD-FP & PAKF \\
\hline \hline Design inlet flow angle $\left(\alpha_{1, \text { des }}\right)\left[^{\circ}\right]$ & 35.0 & 35.0 & 35.0 & 35.0 & 35.0 \\
Design outlet flow angle $\left(\alpha_{2, \text { des }}\right)\left[^{\circ}\right]$ & 60.0 & 60.0 & 60.0 & 60.0 & 60.0 \\
True chord $(\mathrm{B})[\mathrm{mm}]$ & 83.9 & 84.4 & 93.5 & 87.7 & 89.2 \\
Blade span $(\mathrm{h})[\mathrm{mm}]$ & 203.2 & 203.2 & 203.2 & 203.2 & 203.2 \\
Axial chord $\left(\mathrm{B}_{\mathrm{x}}\right)[\mathrm{mm}]$ & 75.3 & 76.0 & 76.0 & 76.0 & 76.0 \\
Pitch spacing $(\mathrm{s})[\mathrm{mm}]$ & 66.8 & 84.2 & 84.2 & 84.2 & 108.5 \\
Stagger angle $(\gamma)\left[{ }^{\circ}\right]$ & 26.0 & 26.0 & 35.4 & 33.4 & 32.9 \\
Trailing edge thickness $[\mathrm{mm}]$ & 1.1 & 1.1 & 1.1 & 1.1 & 1.1 \\
Number of airfoils in cascade & 9 & 7 & 7 & 7 & 6 \\
Design Zweifel coefficient $\left(Z_{w}\right)$ & 1.08 & 1.36 & 1.36 & 1.36 & 1.78 \\
\hline \hline
\end{tabular}

The baseline airfoil, PAKB, is representative of a mid-span cross-section of a low-pressure turbine vane. The cross-section has been inverse-designed so that the non-dimensional pressure distribution at low-speed matches that which is seen under compressible conditions. It was first tested at Carleton University by Mahallati (2003). The midspan performance was documented under steady and unsteady conditions over a range of Reynolds number, freestream turbulence intensity, and turbulence length scale. Surface hot-film measurements were used to study boundary layer transition (Zhang, 2002; Mahallati, 2003), and the use of flow control to suppress stall was investigated (McAuliffe, 2003). PAKB has also been 


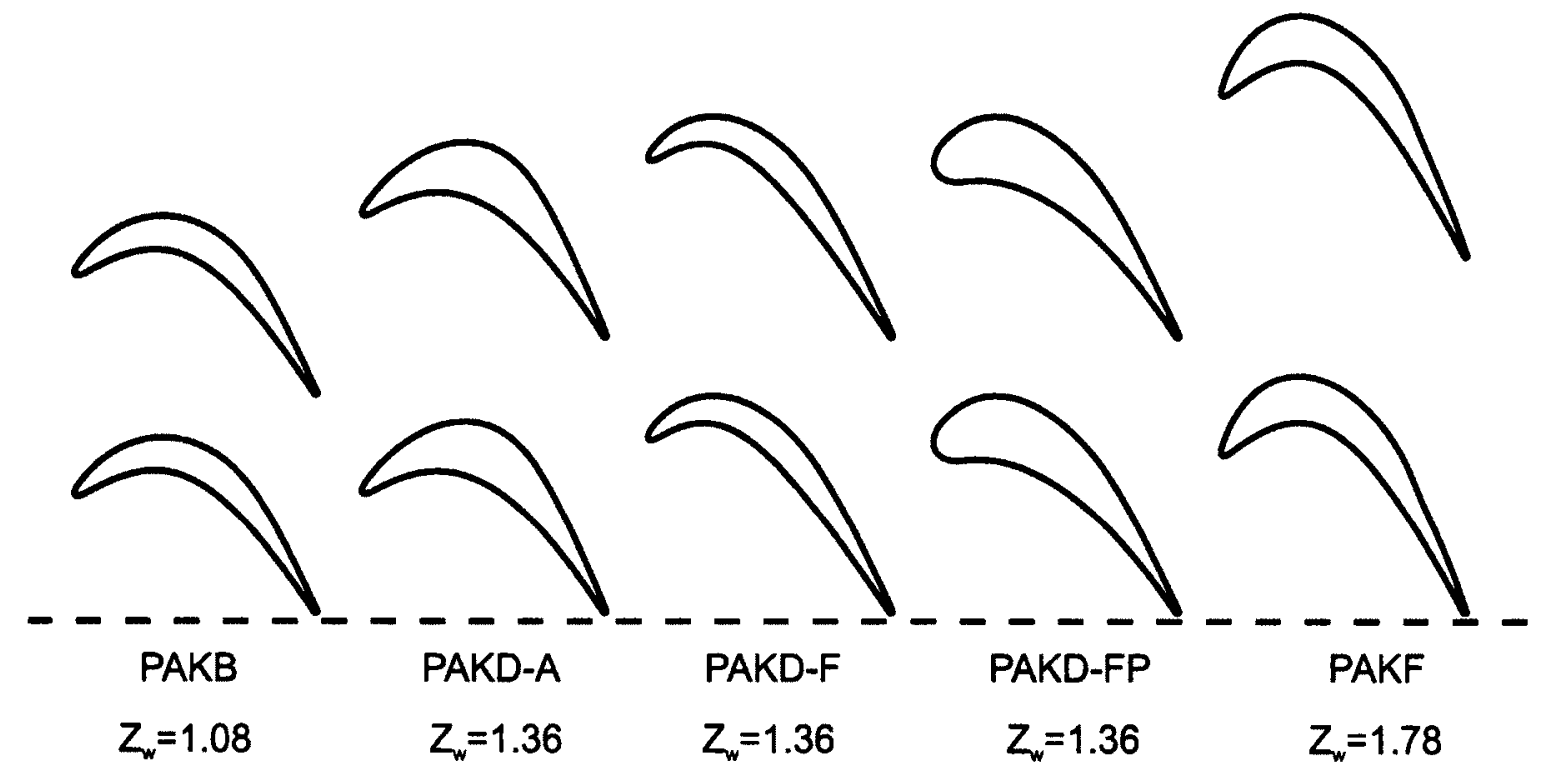

Figure 3.4: Research cascades and respective $Z$ weifel coefficients

tested at the United States Air Force Research Lab, Wright-Patterson, and the United States Naval Academy. Consequently a large body of results exists for this airfoil and the results presented in Chapter 5 supplements the earlier work with blade intra-passage measurements to further document the flow field in the PAKB cascade.

The next generation PAKD airfoils are research specific airfoils designed for testing exclusively at Carleton University. The aft-loaded (PAKD-A) and front-loaded (PAKD-F) were tested by Popović (2005) who measured the midspan performance under steady and unsteady conditions over a range of operating conditions. Concurrently steady secondary flow measurements were conducted by Zorić (2006). Their studies found that PAKD-F is particularly attractive from the perspective of midspan performance, outperforming PAKB for a range of operating conditions. However, it suffers from high secondary losses thus making it a prime candidate for endwall contouring. Chapter 6 investigates the PAKD-F flow field, with and without the application of endwall contouring.

Through the course of this research, two new airfoils have been designed by PWA for the 
study of secondary flows. These are designated PAKD-FP (the pressure-side fix) and PAKF (the airfoil having the highest Zweifel coefficient in open literature to exhibit attached flow).

PAKD-FP was briefly introduced in Section 1.2 where the design intent was described. The airfoil is a redesign of PAKD-F, where the goal was to alleviate the extensive near stagnation of the flow on the pressure-side of the airfoil that results when an attempt is made to minimize the boundary layer edge velocity. The midspan velocity distribution for PAKD-F was presented in Figure 2.10, where it can been seen that the local velocity is less than $10 \%$ of the exit velocity for a considerable fraction of the suction surface length near the leading edge. It was hypothesized that the resulting high static pressure and near stagnation conditions at the pressure-surface/endwall-junction promote strong crosspassage flow in the forward part of the passage, roll-up of the passage vortex, and lead to considerable loss generation. The results for PAKD-FP are discussed in Chapter 7.

The PAKF design had two primary objectives. First objective was to demonstrate the predictive capabilities of current state of the art design tools for creating ultra high-lift blade rows. The airfoil's success relied on the accurate prediction of turbulent transition within the suction surface boundary layer to avoid fully separated stall. The transition model implemented in the design tool is that of Praisner and Clark (2007a). The model was validated with several sets of cascade and engine representative rig data by Praisner and Clark (2007b). PAKF, however, has a loading level greater than the configurations used in the validation and thus demonstrate the potential to extend the useful design space of the tool.

The second objective of PAKF was to establish a flow field which is apt for the study of secondary flows. The strong pressure gradient across the passage promotes the development of a large passage vortex. This exacerbates the physics of the flow and makes them easier to identify. The results of PAKF are presented in Chapter 8. 


\subsection{Cascade Testing for Determination of Entropy Produc- tion}

Thus far the concepts of maximizing the isentropic efficiency and reducing total pressure loss have been used interchangeably but their connection to one another may not be obvious. This section develops the relationship between the two and discusses the use of cascade testing for measuring performance.

Entropy generation is a difficult concept to grasp because entropy can not be measured directly. However, by application of the ideal gas law a change in entropy $\left(s-s_{\text {ref }}\right)$ can be related to the measurable thermodynamic properties of stagnation temperature $\left(T_{o}\right)$ and stagnation pressure $\left(P_{o}\right)$

$$
s-s_{r e f}=C_{p} \ln \left(\frac{T_{o}}{T_{o_{r e f}}}\right)-R \ln \left(\frac{P_{o}}{P_{o_{r e f}}}\right) .
$$

It is important to note that Eq. 3.3 cannot yield an absolute value of entropy only a change from some arbitrary reference value. The efficiency of a turbomachine is dependent only on the change in entropy it produces. Denton (1993) makes the analogy that entropy is like "smoke" that is created within the flow at locations where something harmful to the machine's performance is occurring. Once created the smoke cannot be destroyed, but it defuses and is convected downstream. At the exit of the machine, the average concentration of "smoke" contains a contribution from all upstream sources, and is proportional to the degradation in performance.

For adiabatic flow through a stationary blade row such as a turbine inlet guide vane or a linear cascade, no work is extracted from the flow so that the stagnation temperature 
remains constant. Thus Eq. 3.3 reduces to

$$
\Delta s=-R \ln \left(\frac{P_{o 2}}{P_{o 1}}\right)
$$

where $P_{o 1}$ and $P_{o 2}$ are the stagnation pressures at the inlet and outlet of the blade row respectively. If the variation in total pressure across the blade row is small, Eq. 3.4 can be approximated as

$$
\Delta s=-R \frac{\left(P_{o 2}-P_{o 1}\right)}{P_{o 1}}
$$

The loss associated with this change in entropy can be interpreted as the amount of energy no longer available to perform work in the subsequent rotor stage. Equivalently it is the difference in energy between the actual conditions and isentropic conditions downstream of the stationary blade row. Following Taylor (1971), this energy per unit mass, or specific work $(w)$ is defined as

$$
w=\oint T \cdot d s=T_{o 1} \cdot \Delta s=-R T_{o 1} \frac{\left(P_{o 2}-P_{o 1}\right)}{P_{o 1}}
$$

Using the ideal gas relationship $R T_{o 1}=P_{o 1} / \rho_{o 1}$, Eq. 3.6 can be rewritten

$$
w=-\frac{1}{\rho_{o 1}}\left(P_{o 2}-P_{o 1}\right)
$$

Equation 3.5 states that the entropy generated through a stationary blade row is proportional to the total pressure difference from the inlet to the outlet, whereas Eq. 3.7 relates the energy lost due to entropy generation to the same pressure difference. These concepts make linear cascade testing a convenient tool to assess blade row performance. Taking total pressure measurements in a plane both upstream and downstream of the cascade 
gives sufficient information to quantify the loss generated in turning the flow. The inlet measurements are used to quantify the momentum deficit in the incoming boundary layer, while the downstream measurements account for the loss accumulated. In fact, the plane on which $P_{o 2}$ is measured need not be downstream of the trailing edge at all. Several investigators have followed the lead of Langston et al. (1976) using intra blade passage measurements to quantify loss as a function of axial distance downstream of the trailing edge.

In addition to effective quantification of losses, linear cascade testing offers an idealized environment for the investigation of loss generation mechanisms. Cascade tests lack the complexity of unsteadiness due to relative motion found in rotating rigs and there is no centrifugal force to impose a pressure gradient in the spanwise direction. Although these phenomena play an important role influencing the flow within an engine, it is often desired to study a simplified configuration to develop a deeper understanding of the flow physics. Despite its simplifications cascade tests present a reasonably accurate representation of flow structures and offer a systematic and repeatable manner in which to assess them.

\subsection{Experimental Instrumentation}

During the course of this research the data acquisition system was refurbished by the present author and Tanya Ellison (Ellison, 2005). Much of the original infrastructure was salvaged from the existing system developed by Mahallati (2003). New data acquisition software and a graphical user interface was developed using the LabView visual programming language. 


\subsubsection{Data Acquisition}

Instrument voltage output was sampled using a United Electronics Industries PowerDAQ PD2-MFS-8-800/14 board. The card mounts in the PCI slot of the data acquisition computer's mother board. It has eight single ended simultaneous sample and hold analogto-digital converter (ADC) channels. The board has an ADC resolution of 14 bit and a maximum sampling rate of 800,000 samples per second. Further details regarding the workings of the data acquisition board are given by Mahallati (2003). During the initial shakedown of the system, performance of the board was tested extensively using a signal generator to ensure that it maintained signal integrity.

\subsubsection{Pressure Transducers}

Pressures were measured with Data Instruments ASG DRAL501DN and DRAL505DN differential transducers having an operating range of \pm 250 and \pm 1250 Pascals, respectively. The transducers offer temperature compensation over the range of 0 to +50 degrees centigrade. The estimated uncertainty in pressure measurement due to calibration, linearity, and hysteresis is $\pm 0.5 \mathrm{~Pa}$ for the DRAL501DN and $\pm 2 \mathrm{~Pa}$ for the DRAL505DN.

Transducers were calibrated using a water micromanometer described by Benner (2003). The micromanometer is used to expose the differential transducers to pressure difference equivalent to a known height of water. The voltage output $(E)$ from the transducer is linear in response to a pressure difference $(\Delta P)$ and follows the form

$$
\Delta P=C_{1} \cdot E+C_{2}
$$

where $C_{1}$ is the calibrated slope of the voltage response and $C_{2}$ is the zero pressure offset voltage which is corrected for prior to and after each test. Sample calibration data for the 
pressure transducers are presented in Appendix A

\subsubsection{Temperature Monitor}

Static temperature is measured with a type- $T$ (Copper-Constantan) thermocouple secured near the lower bypass of the test section. The thermocouple voltage is discretized to 15 bits accuracy by an Omega i-series temperature/process meter DPi8-C24 at a sampling frequency of $3 \mathrm{~Hz}$. Mahallati (2003) quotes the accuracy of the temperature measurement as $\pm 0.4^{\circ} \mathrm{C}$. The temperature meter connects to the data acquisition computer via the USB port and the data are logged during the course of testing. The static temperature is used to compute the dynamic viscosity of the air by applying Sutherland's rule.

\subsubsection{Digital Barometer}

Atmospheric pressure is measured using an Omega digital barometer, model number DPI740. The instrument has an operating range of 77 to $115 \mathrm{kPa}$ absolute pressure. Pressures are resolved to $1.016 \mathrm{~Pa}$ and sampled at $2 \mathrm{~Hz}$. The manufacturer's quoted measurement uncertainty is $\pm 0.02 \%$ of full scale range. Barometric readings are transmitted to the data acquisition computer through the RS-232 port and are used to compute the density of the air in the test section using the ideal gas law. Ideally the static pressure in the test section would be used for the calculation, but this would require an extra pressure transducer. During the initial shakedown of the system, the static pressure in the test section was measured relative to atmospheric pressure and was found to be roughly $200 \mathrm{~Pa}$ higher for a range of operating conditions. This corresponds to a negligible underestimation of the Reynolds number by 250 (based on inlet velocity and axial chord) when atmospheric pressure is used in the calculation. 


\subsubsection{Stepper Motor Controller}

The spanwise and pitchwise position of the probe is automated during the course of a test with the use of a two-axis linear traverse mechanism. The traverse gear is driven by two stepper motors directed by a Velmex VXM stepping motor controller. The controller is capable of moving the motors one at a time. 400 steps are required to complete a full revolution and each step sent to the motor moves the probe by $0.003175 \mathrm{~mm}$. During the commissioning of the data acquisition system the speed of the traverse was set to ensure that steps were not missed by the motor. The controller receives ASCII commands from the data acquisition computer via an RS-232 interface cable.

\subsection{Probe Calibration Apparatus}

Probes were calibrated in-house using the calibration apparatus shown schematically in Figure 3.5. The motorized turnable mechanism designed by Yaras (1987) is contained in an open-circuit, suction-type calibration box developed by Benner (2003). Air is drawn through the apparatus from right to left initially passing commercial air filter panels. The panels prevent dust and aerodynamic disturbances from entering the setup. The flow passes through a bell mouth and is accelerated smoothly through a nozzle having a diameter $(d)$ of $55 \mathrm{~mm}$. The jet leaving the nozzle passes over the axes of rotation of the turntable mechanism and leaves through a second bell mouth. The setup is powered with a radial blower having a throttle valve that can set the operating point between $1 \mathrm{~m} / \mathrm{s}$ and $45 \mathrm{~m} / \mathrm{s}$.

The probe holder secures the probe such that the head passes through both the pitch and yaw axes of rotation. This ensures that the head remains in the same location as the probe is calibrated in the inviscid core of the jet. The turntable mechanism is powered by two stepper motors and provides $\pm 70^{\circ}$ of probe rotation in both directions with an estimated 
accuracy of $\pm 0.01^{\circ}$ and negligible backlash. The calibration jet has been well documented. Variations in total and static pressure across the inviscid core are on the order of $\pm 0.1 \%$ of centerline dynamic pressure with angle variations within $\pm 0.4^{\circ}$ (Benner, 2003). The turbulence intensity within the core is $0.4 \pm 0.1 \%$ over the operating range of jet velocities (Mahallati, 2003).

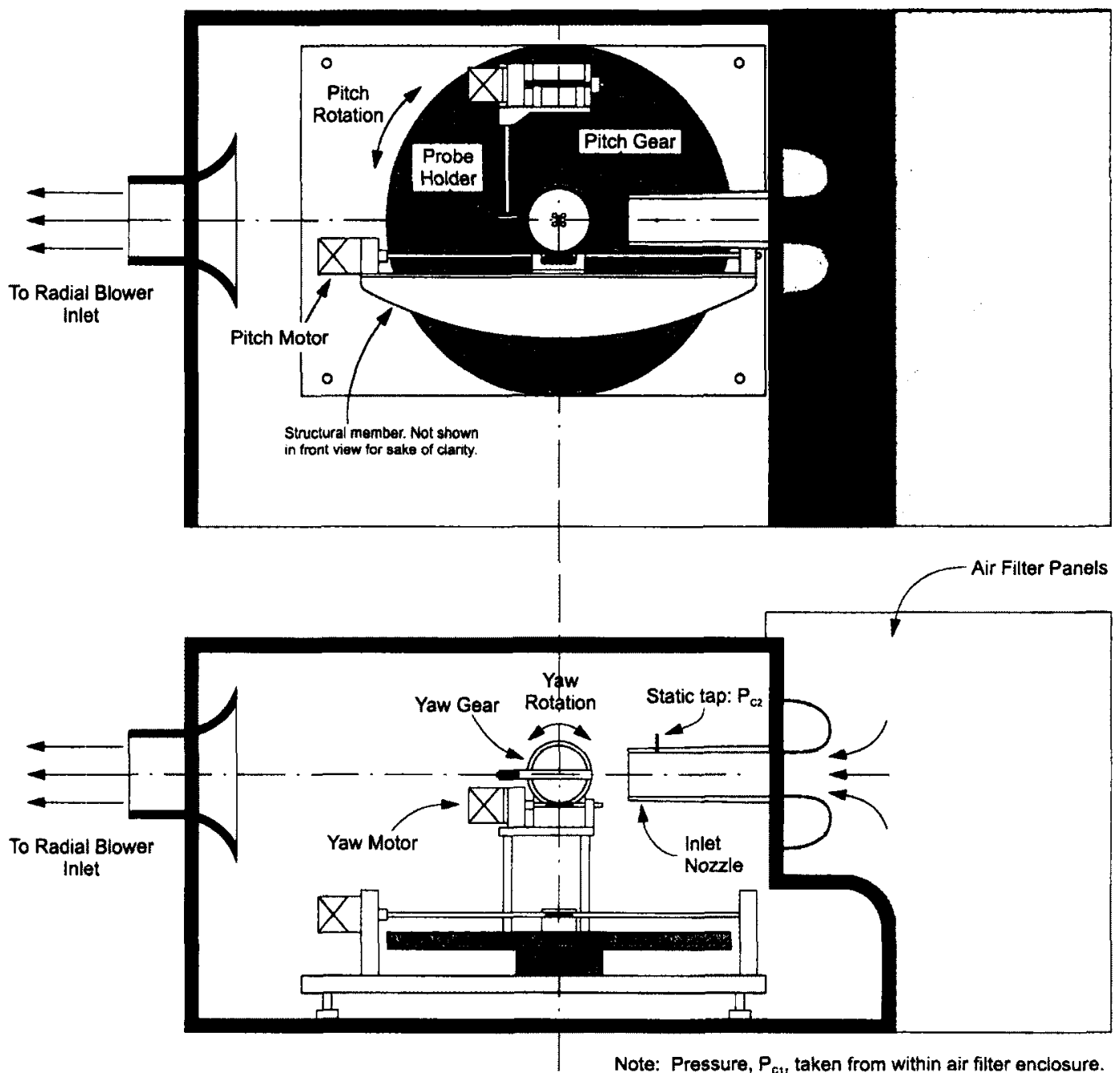

Figure 3.5: Schematic of probe calibration apparatus, reproduced from Benner (2003)

The reference total pressure $\left(P_{C 1}\right)$ was measured inside the air filter enclosure where the air is essentially stagnant. The reference static pressure $\left(P_{C 2}\right)$ was measured with a static 
tap located just upstream from the nozzle exit.

\subsection{Experimental Uncertainty}

Any experiment is prone to measurement errors. Thus it is necessary to carry out an uncertainty analysis to assess the quality of the data. Several approaches to uncertainty analysis exist in open literature but the single-sample uncertainty method of Moffat (1982) is most suitable for wind tunnel measurements.

Statistically, the uncertainty of a measured value $(x)$ can be written as

$$
\delta x= \pm 2 \sigma
$$

where $\sigma$ is the standard deviation of the data. If the data follows a Gaussian distribution, then $\delta x$ represents the bandwidth within which the experimenter is $95.4 \%$ confident that the true value lies.

The method of Moffat (1982) separates experimental errors into two categories, random and bias. Bias errors are introduced through instrument calibration errors. This experiment follows a long lineage of researchers who have established procedures to hone the bias errors to low values and quantify them. Random errors occur as a result of signal discretization,

probe misalignment, interpolation, repeatability, etc. A quantity, $X$ is influenced by individual sources of error such that

$$
X=f\left(x_{1}, x_{2}, \ldots, x_{N-1}, x_{N}\right)
$$

where $N$ is the total number of error sources. The effect of each error source on $X$ can be 
found by computing the relative perturbation sensitivity:

$$
\delta X_{n}=\left(\frac{\partial X}{\partial x_{n}} \delta x_{n}\right)
$$

The partial derivatives can be evaluated directly, or by independently perturbing each error source within its error band to determine the effect on $X$. Since all error sources do not achieve their maximum values simultaneously, and because some of the partial derivatives may result in negative slopes, it is appropriate to take the root sum square of the perturbation sensitivities to determine the uncertainty of a quantity $(\delta X)$ :

$$
\delta X= \pm \sqrt{\sum_{n=1}^{N}\left(\frac{\partial X}{\partial x_{n}} \delta x_{n}\right)^{2}}
$$

The uncertainty for each type of measurement in this study will be quoted in connection with the description of the instrument used to measure it below.

\subsection{Pressure Probes}

\subsubsection{Pitot Tube}

The inlet boundary layer was traversed $1.2 B_{x}$ upstream of the leading edge of the cascade with a Pitot tube having inner and outer diameters of 0.33 and $0.64 \mathrm{~mm}$ respectively. The stainless steel tube is goosenecked such that the tip contacts the wall first as it brought toward the surface allowing the probe's zero position to be determined aerodynamically. The total pressure measured by the Pitot probe has an estimated uncertainty of $\pm 0.3 \%$ of inlet midspan dynamic pressure. 


\subsubsection{Three-Hole Probe}

Throughout the course of this work, two-dimensional flow fields were documented with a three-hole pressure probe having a wedge angle of $45^{\circ}$. The probe, shown in Figure 3.6 has a tip with a thickness of $0.64 \mathrm{~mm}$ and a width of $2 \mathrm{~mm}$. Prior to use in an unknown flow, the probe must be calibrated. The three-hole probe was calibrated over a range of pitch angles spanning $\pm 21^{\circ}$ in $0.5^{\circ}$ increments. The pitch angle is measured in a plane that bisects the three pressure ports. Positive pitch is defined as the orientation where the flow is directed toward hole 3 rather than hole 2. The calibration apparatus was discussed in Section 3.7 and the calibration procedure and sample calibration data are presented in Appendix B.

Three-hole probes have been used extensively by previous researchers in the LSTL. A detailed discussion of the experimental uncertainty is given by Benner (2003). In a threedimensional flow, a yaw misalignment of the incoming velocity vector results in a lower total pressure being recovered by the probe that cannot by corrected through calibration. The yaw angle is measured in a plane normal to the plane that contains the pitch angle. Mahallati (2003) tested the sensitivity of the probe to yaw angles in increments of $2.5^{\circ}$. It was determined that the calibration was insensitive to misalignment in the yaw direction of $\pm 5^{\circ}$

Measured pitch angles have an estimated uncertainty of $\pm 0.4^{\circ}$. Dynamic and total pressures measured have uncertainties of $\pm 0.6 \%$ and $\pm 0.3 \%$ of inlet midspan dynamic pressure, respectively. 

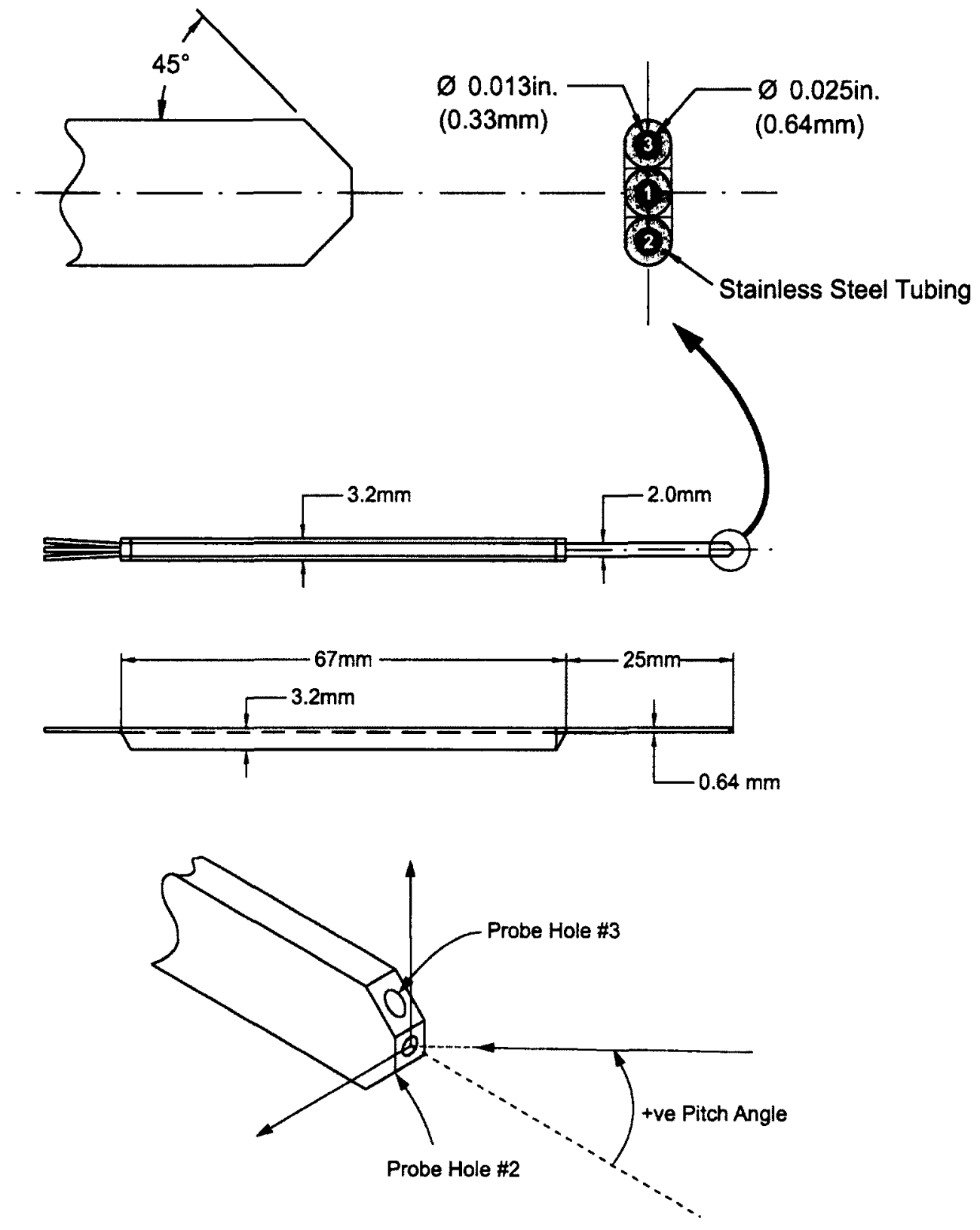

Figure 3.6: Three-hole pneumatic probe, reproduced from Benner (2003)

\subsubsection{Seven-Hole Probe}

Three-dimensional flow fields were documented using a seven-hole pressure probe. To facilitate intra-passage measurements near the endwall, a new gooseneck seven-hole probe 
and mounting system was designed. The new probe, shown schematically in Figure 3.7 has the same head geometry used in the previous secondary flow studies conducted at Carleton University. It has a $60^{\circ}$ cone angle but uses smaller hypodermic tubing resulting in a smaller head diameter compared to the probes used by Benner (2003) and Zorić (2006) (1.83 mm vs. $2.4 \mathrm{~mm})$.

The new probe mounting assembly allows the axial position of the probe to be adjusted independently of the pitchwise and spanwise alignment or position. Thus the angular misalignment errors between measurement planes are negligible and the position of the traverse planes relative to one another is known. The probe was calibrated in $2^{\circ}$ increments over a range of $\pm 50^{\circ}$ of misalignment in both pitch and yaw. Details of the calibration and data reduction procedures are given in Appendix C.

The estimated uncertainty for measured flow angles is $\pm 0.5^{\circ}$ and uncertainties in measured dynamic and total pressure are estimated to be $\pm 1.1 \%$, and $\pm 0.4 \%$ of inlet midspan dynamic pressure, respectively. The seven-hole probe data were sampled at a frequency of $1 \mathrm{kHz}$ for 15 seconds.

\subsection{Cascade Testing Procedures}

\subsubsection{Wind Tunnel Calibration and Operating Point}

To operate the wind tunnel, the test section must be calibrated so the cascade inlet conditions can be related to the contraction pressure difference $P_{C 1}-P_{C 2}$ (recall Figure 3.1). The inlet centerline is located on the axial measurement plane $1.2 B_{x}$ upstream of the leading edge of the cascade. The wind tunnel total pressure calibration coefficient $C_{P o_{W T}}$ is defined then as:

$$
C_{P o_{W T}}=\frac{P_{o}-P_{C 1}}{P_{C 1}-P_{C 2}}
$$



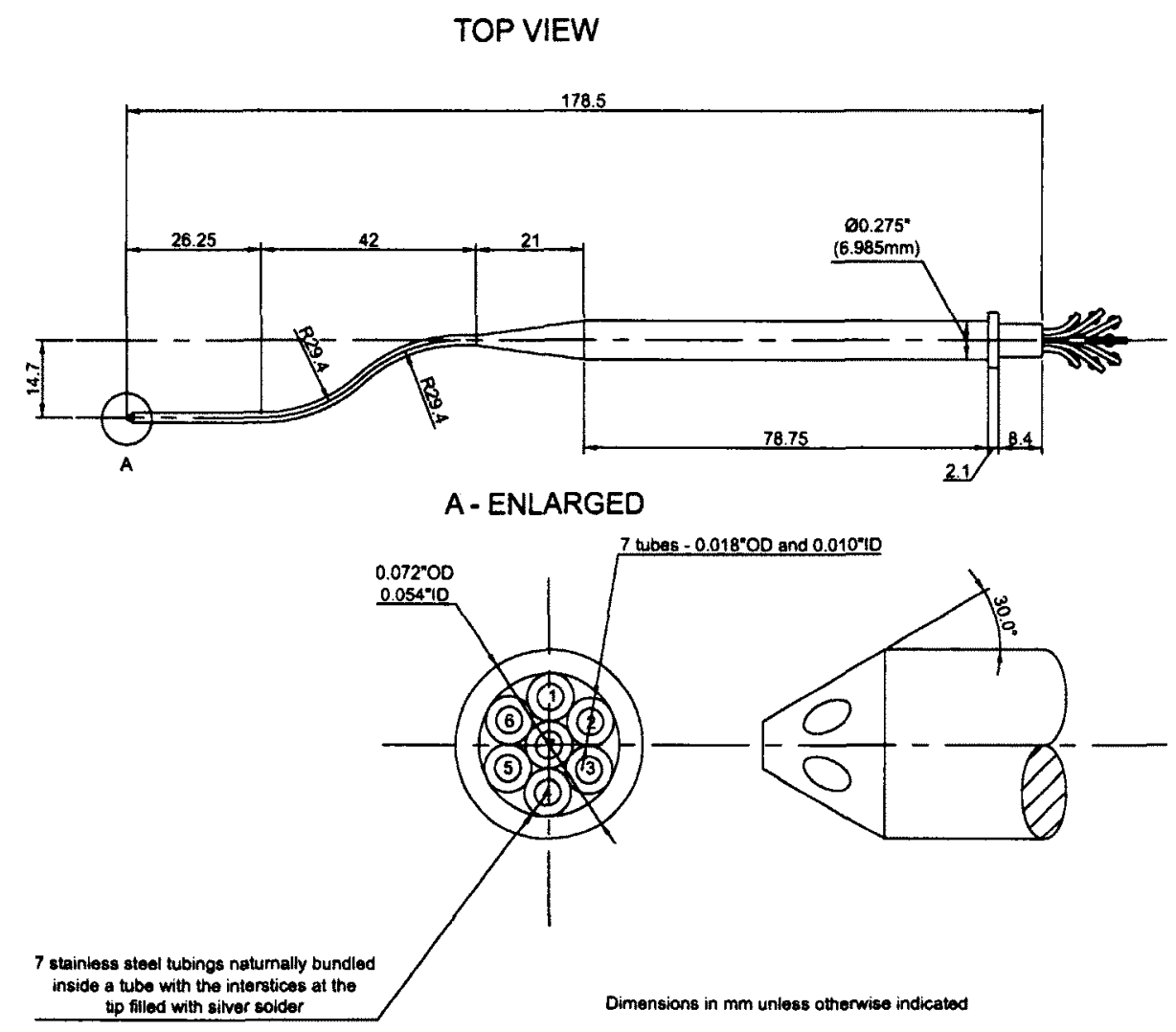

Figure 3.7: Seven-hole pneumatic probe

where $P_{o}$ is the total pressure on the inlet centerline of the cascade. The wind tunnel dynamic pressure calibration coefficient $\left(C_{q_{W T}}\right)$ is defined as:

$$
C_{q_{W T}}=\frac{P_{o}-P}{P_{C 1}-P_{C 2}}
$$

where $P$ is the static pressure on the inlet centerline of the cascade. The wind tunnel operating point is set to match a specific Reynolds number $(R e)$ base on cascade inlet centerline velocity $\left(V_{C L}\right)$ and blade axial chord $\left(B_{x}\right)$

$$
R e=\frac{\rho \cdot V_{C L} \cdot B_{x}}{\mu}
$$


where $\rho$ is the air density found by ideal gas law (Section 3.6.4) and $\mu$ is the air kinematic viscosity found by Sutherland's rule (Section 3.6.3). The inlet centerline velocity is related to the wind tunnel dynamic pressure calibration coefficient as follows:

$$
V_{C L}=\sqrt{\frac{2 \cdot\left(P_{C 1}-P_{C 2}\right) \cdot C_{q_{W T}}}{\rho}} .
$$

The design Reynolds number for the PAK family of airfoils is $8.0 \times 10^{4}$ based on inlet midspan velocity and axial chord. At this condition, PAKB and PAKD-A have midspan suction surface separation bubbles which interact with the secondary flow and influence loss generation. In order to reduce this effect, and improve the comparability of the results from one cascade to the next, an elevated Reynolds number was used for the endwall contouring study. The current investigation was conducted at design incidence and a Reynolds number of $1.26 \pm 0.02 \times 10^{5}$ based on inlet midspan velocity and axial chord. The inlet midspan velocity was approximately $25 \mathrm{~m} / \mathrm{s}$. Mach numbers at the inlet and outlet were approximately 0.073 and 0.112 , respectively; thus the flow in the test section was essentially incompressible.

The Reynolds number based on inlet velocity is used for convenience to set the experimental operating point. However, for turbine blade flows the Reynolds number based on outlet velocity is most relevant. Using the outlet velocity, the design Reynolds number for the PAK family is $1.24 \times 10^{5}$ and the elevated Reynolds number used for the current testing was $1.95 \times 10^{5}$. 


\subsubsection{Data Reduction}

\subsubsection{Measured and Mixed-Out Losses}

In Section 2.2 three categories were discussed to subdivide the losses generated in a turbine blade passage. Loss data presented for turbines is typically expressed in terms of the mass-averaged total pressure loss coefficient $\left(Y_{\text {total }}\right)$, which is a function of these three categories of losses. The mass-averaged total pressure loss coefficient is then defined as:

$$
Y_{\text {total }}=Y_{\text {profile }}+Y_{\text {secondary }}+Y_{\text {tip }}
$$

where $Y_{\text {profile }}, Y_{\text {secondary }}$ and $Y_{\text {tip }}$ are the mass-averaged profile, secondary, and tip clearance loss coefficients, respectively. The current research is conducted in the absence of tip clearance, so that $Y_{\text {tip }}$ can be omitted. $Y_{\text {total }}$ is obtained experimentally through the total pressure deficit coefficient, $C_{P o}$.

$$
C_{P o}=\frac{P o-P o C L_{1}}{q_{C L_{1}}}
$$

where $P_{O}$ is the local total pressure measured and $P_{O C L_{1}}$ and $q_{C L_{1}}$ are the mass-averaged total and dynamic pressures on the inlet centerline of the cascade. Measurements are made on axial planes and the results are expressed in terms of the total pressure deficit coefficient. Total pressure is related to entropy (Section 3.5), which is an extensive flow property. Thus it is appropriate to mass-average $C_{P o}$ on an axial plane to determine the loss that exists there. Mass-averaging of the total pressure deficit coefficient is accomplished as follows:

$$
C_{P_{0}}^{\prime \prime}=\frac{\int_{0}^{h / 2} \int_{0}^{s}\left(\rho u(y, z) C_{P o}(y, z)\right) \mathrm{d} y \mathrm{~d} z}{\int_{0}^{h / 2} \int_{0}^{s}(\rho u(y, z)) \mathrm{d} y \mathrm{~d} z}
$$


The integration limits are set from the wall to half span and across one blade pitch in the $y$ direction. The intra-blade passage measurements are evaluated to their extents as was limited by probe positioning in the $y$ direction. Node centered integration is used in the data reduction routine. A wall treatment was applied to the endwall and to the blade suction and pressure surfaces within the blade passage. The total pressure was assumed to be the same as the static pressure measured at the nearest node to the wall. At the wall, the velocity was set to zero.

The denominator of Equation 3.19 represents the mass-flux through the measurement plane, which is defined by the axial velocity. The mass-flux can be used to verify the consistency of the data. That is, one would expect the same value at each downstream measurement plane that was measured at the inlet (1.2B $B_{x}$ upstream of the cascade). For the intra-blade passage measurements the probe positioning was such that the blade suction surface and pressure surface boundary layers were not captured in their entirety. At these planes, a deficit of mass flow on the order of $0.5 \%-0.8 \%$ was measured relative to the inlet. In light of this, the mass-averaged loss values for these planes are not quoted, and these measurements are used strictly to gain insights into the development of the secondary flow within the passage. At the measurement planes downstream of the trailing edge the largest error in mass-flux relative to the inlet plane was about $\pm 0.3 \%$.

The overall total pressure loss coefficient $\left(Y_{\text {total }}\right)$ is related to $C_{P_{0}}^{\prime \prime}$ using

$$
Y_{\text {total }}=\left(C_{P_{o_{1}}}^{\prime \prime}-C_{P o_{2}}^{\prime \prime}\right)\left(\frac{q_{C L}}{q_{2}}\right)^{\prime \prime}
$$

where the subscripts 1 and 2 denote the inlet and downstream measurement planes, respectively. As was noted above, the reference inlet plane is located $1.2 B_{x}$ upstream of the leading edge of the cascade. The nominal downstream measurement plane is found $1.4 B_{x}$ downstream of the leading edge. The dynamic pressure $(q)$ is defined as $q=1 / 2 \rho V^{2}$. 
$Y_{\text {total }}$ represents the row loss incurred in turning the flow through the cascade. This study is focused on controlling secondary losses in turbine cascades and as such the secondary loss coefficient $\left(Y_{\text {secondary }}\right)$ is of particular interest. $Y_{\text {secondary }}$ is found by subtracting the profile loss coefficient ( $Y_{\text {profile }}$ ) from $Y_{\text {total }}$ (rearranging Equation 3.17 and neglecting $Y_{\text {tip }}$ ). The profile loss coefficient is found through pitchwise-mass-averaging the total pressure deficit coefficient at midspan as follows:

$$
C_{P o}^{\prime}=\frac{\int_{0}^{s}\left(\rho u(y, z) C_{P o}(y, z)\right) \mathrm{d} y}{\int_{0}^{s}(\rho u(y, z)) \mathrm{d} y}
$$

where the integration limit in the y-direction is one blade pitch. $Y_{\text {profile }}$ is then found by applying Equation 3.21 at midspan in the relation:

$$
Y_{\text {profile }}=C_{\text {Poprofile }}^{\prime}\left(\frac{q_{C L}}{q_{2}}\right)^{\prime}
$$

The loss coefficients define thus far refer to measured losses that have been incurred turning the flow between in the inlet boundary layer traverse plane and the downstream measurement plane. At the downstream plane, the flow is spatially non-uniform and additional losses will be generated as the flow mixes to a uniform condition. The condition where the flow is spatially uniform is referred to as the mixed-out condition. In order to allow comparisons of data between facilities, where downstream measurements may have been taken at different downstream axial locations, the mixed-out losses are used. At midspan, the mixed-out losses are independent of downstream measurement location, and it is for this reason that they are used for correlation purposes.

Mixing losses are calculated by applying the continuity and momentum equations to a control volume that extends one blade pitch, from the wall to half-span, between the downstream measurement plane and the fully mixed-out plane. The mixing is assumed to 
occur at constant area under incompressible conditions. The momentum balance neglects the influence of the endwall shear stress, which produces additional loss as a result of viscous dissipation. However, Moore and Adhye (1985) found that the contribution of wall shear stress to loss was small downstream of $140 \% B_{x}$. Also, Yaras and Sjolander (1989) determined that the contribution of the endwall shear stress could not completely account for the increase in mixed-out loss from subsequent measurement planes upstream of $140 \%$ $B_{x}$. The details of the mixing calculations for overall row and profile loss are presented in Section 3.9.1 of Benner (2003). The final form of the mixed-out losses are identical to Equations 3.20 and 3.22 except that the downstream condition (2) is replaced by the hypothetical mixed-out plane.

\subsubsection{Streamwise Vorticity, Helicity and Secondary Kinetic Energy}

The three-dimensional flow structures in the endwall region can be interpreted by examining the streamwise vorticity, helicity, and secondary kinetic energy. These three parameters are defined here.

At any point in a flow, the vorticity is defined as the curl of the velocity vector, or

$$
\omega=\nabla \times V
$$

In cartesian coordinates the axial, pitchwise and spanwise components of the vorticity vector are defined as follows:

$$
\begin{gathered}
\omega_{x}=\frac{\partial w}{\partial y}-\frac{\partial v}{\partial z} \\
\omega_{y}=\frac{\partial u}{\partial z}-\frac{\partial w}{\partial x} \approx \frac{1}{u}\left(v \omega_{x}+\frac{1}{\rho} \frac{\partial P_{o}}{\partial z}\right) \\
\omega_{z}=\frac{\partial v}{\partial x}-\frac{\partial u}{\partial y} \approx \frac{1}{u}\left(w \omega_{x}-\frac{1}{\rho} \frac{\partial P_{o}}{\partial y}\right)
\end{gathered}
$$


In order to extract the vorticity from a single axial measurement plane, the approximations indicated in Equations 3.25 and 3.26 are used. The formulations are derived from the incompressible Euler equations as per Gregory-Smith et al. (1988). The full derivation is available in Section 5.2 of Yaras (1990) who investigated the validity of the simplifications relative to the actual vorticity as determined by measuring two closely spaced planes. He found that the single-plane approximation gave nearly identical values for the vorticity components everywhere but where the velocity gradient was changing most rapidly. This corresponds to regions of the flow where viscosity plays a significant role, and the Euler equations would by definition fail to capture the effect. The maximum local error for the single-plane method was within $10 \%$ that of the two-plane approach.

The streamwise component of vorticity takes the form

$$
\omega_{s}=\omega_{x} \cos \alpha_{2}^{\prime \prime}+\omega_{y} \sin \alpha_{2}^{\prime \prime}
$$

where $\alpha_{2}^{\prime \prime}$ is the mass-averaged outlet flow angle defined by as

$$
\alpha_{2}^{\prime \prime}=\tan ^{-1}\left(\frac{\iint\left(\rho u_{2} v_{2}\right) \mathrm{d} y \mathrm{~d} z}{\iint\left(\rho u_{2}^{2}\right) \mathrm{d} y \mathrm{~d} z}\right)
$$

The non-dimensional streamwise vorticity coefficient is defined as:

$$
C_{\omega_{s}}=\frac{\omega_{s} C}{V_{C L}}
$$

where $C$ is the airfoil chord.

Helicity defines the extent to which a corkscrew motion occurs within a fluid. That is, a parcel of fluid has helicity if it undergoes solid body rotation about an axis in line with the direction of motion. Both vorticity and helicity are conserved quantities in fluids obeying 
the Euler equations so they can be used to track endwall flow structures. By definition, the helicity is the dot product of the velocity with the vorticity, or

$$
H_{e}=V \cdot(\nabla \times V)=u \omega_{x}+v \omega_{y}+w \omega_{z}
$$

The non-dimensional coefficient of helicity is defined as follows:

$$
C_{H_{e}}=\frac{H_{e} C}{V_{C L}^{2}}
$$

The secondary kinetic energy is the kinetic energy associated with the secondary velocity vectors. The secondary velocity vectors are the components of velocity decomposed on a plane perpendicular to the mass-averaged flow angle $\left(\alpha_{2}^{\prime \prime}\right)$. Thus the secondary kinetic energy $(S K E)$ is expressed as

$$
S K E=\frac{1}{2} \rho\left(v_{s e c}^{2}+w_{s e c}^{2}\right)
$$

where $v_{\text {sec }}=-u_{2} \sin \alpha_{2}^{\prime \prime}+v_{2} \cos \alpha_{2}^{\prime \prime}$ and $w_{\text {sec }}=w_{2}$. Then the non-dimensional coefficient of secondary kinetic energy $\left(C_{S K E}\right)$ is defined as

$$
C_{S K E}=\frac{v_{s e c}^{2}+w_{s e c}^{2}}{V_{C L}^{2}}
$$

Downstream of a linear cascade the secondary kinetic energy is dissipated through viscosity and is irrecoverable loss. That is, for two blade rows having the same total-pressure loss at a given downstream axial location, the row generating less secondary kinetic energy would have lower mixed out loss (if diffusion loss is ignored). The secondary kinetic energy is not necessarily irrecoverable loss in an actual turbomachine. It is arguable that some of the secondary kinetic energy could be recovered in the downstream rotor. 


\subsubsection{Inlet and Outlet Flow Quality}

Significant care was taken to set the inlet uniformity and outlet periodicity prior to taking detailed measurements. This was essential so that the flow through the central passages of the cascade mimicked that of an infinitely repeating blade row. Initially the tailboards were set slightly divergent relative to the design outlet flow angle. The inlet uniformity was then adjusted with the flaps and blockage mechanism (see Figure 3.2). After each adjustment, the inlet centerline of the cascade was documented $1.2 B_{x}$ upstream of the leading edge with the three-hole pressure probe described in Section 3.9.2. The maximum extent of the inlet traverse gear was sufficient to capture the flow across four blade pitches for the PAKB and PAKD airfoils. Once the inlet flow was aligned with the design inlet angle, the probe was moved to the outlet and the outlet centerline was documented $1.4 B_{x}$ downstream of the leading edge of the cascade. The outlet periodicity was set using the tailboards.

The uniformity and periodicity are coupled through the area between the top flap and the top tailboard. As such, the initial setup requires several iterations moving the probe from the inlet to the outlet and back before the flow was periodic over the central passages of the cascade.

A sample of the uniformity and periodicity data is presented in Figure 3.8. The inlet and outlet flow angles are presented, along with the dynamic, static, and total pressure coefficients referenced to the wind tunnel contraction pressures. The variation in flow angle is within probe measurement error across four blade pitches.

The turbulence generating grid was installed 18.4 axial chords upstream of the middle of the cascade. The grid produces a freestream turbulence intensity of $4 \%$ with an integral length scale of $0.4 B_{x}$ (Mahallati, 2003). Gregory-Smith and Cleak (1992) studied the effects of freestream turbulence intensity on secondary flows and found little influence on secondary 

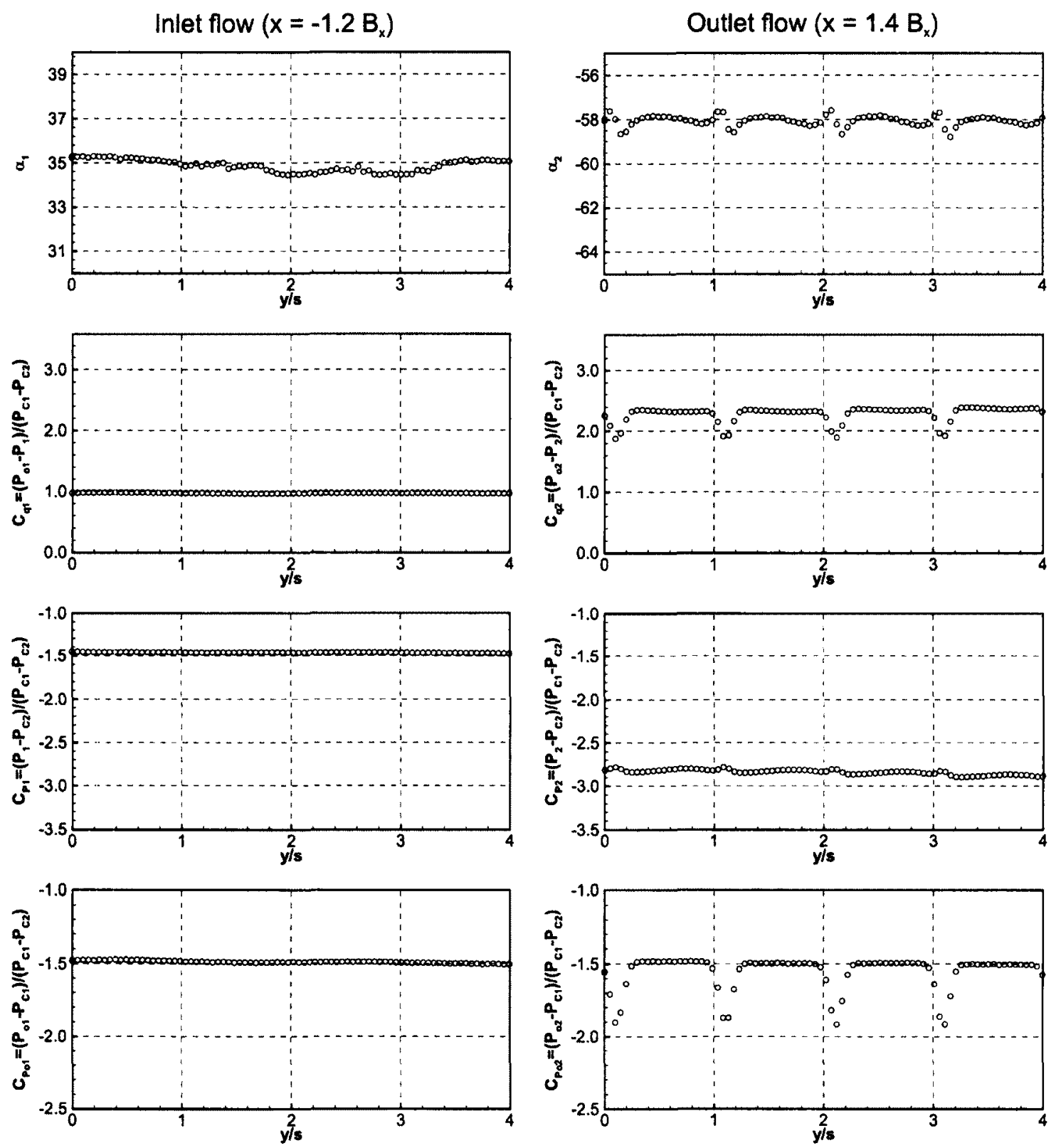

Figure 3.8: Inlet uniformity and outlet periodicity - PAKB

loss and secondary kinetic energy. Zorić (2006) conducted a similar study investigating the effects of turbulence intensity on row loss for the PAKB and PAKD airfoils. As found by Gregory-Smith and Cleak (1992), installing the turbulence generating grid resulted in a thinner inlet boundary layer and led to slightly lower secondary losses. Based on 
these studies it is suggested that the secondary flow physics discussed herein are relatively insensitive to the level of freestream turbulence intensity.

Once the flow through the cascade is set it is necessary to document the inlet boundary layer to determine its state and to quantify the total pressure deficit. The inlet was traversed at several pitchwise locations with the Pitot-tube described in Section 3.9.1. The results of three traverses are shown in Figure 3.9a: one traverse located at mid-pitch and two at $25 \%$ to either side. The turbulence generating grid used in the present study caused a nonuniform total pressure distribution between the endwall and midspan. A similar inlet total pressure distribution was reported by Hartland et al. (2000) for their upstream mounted passive turbulence generating grid. To account for the non-uniformities caused by the grid, the inlet was traversed with a three-hole pressure probe. The results of this traverse is shown in Figure $3.9 \mathrm{~b}$. These results are mass-averaged over one blade pitch from the wall to half span to determine the reference total pressure coefficient $\left(C_{P o, 1}^{\prime \prime}\right)$. All inlet measurements were sampled for 10 seconds at $1 \mathrm{kHz}$.

The range of integrated boundary layer parameters for the three Pitot-tube traverses is presented in Table 3.3. The low boundary layer shape factor $(H)$ is typical for a turbulent boundary layer with zero pressure gradient. The boundary layer thickness $(\delta)$ is engine representative for a low-pressure turbine (Private Communication, PWA).

Table 3.3: Range of inlet boundary layer parameters and mass-averaged $C_{P o}$

\begin{tabular}{ll}
\hline \hline$\delta / h$ & $0.16-0.16$ \\
$\delta^{*}[\mathrm{~mm}]$ & $2.0-2.3$ \\
$\theta[\mathrm{mm}]$ & $1.7-1.9$ \\
$H$ & $1.20-1.21$ \\
$C_{P_{0,1}}^{\prime \prime}$ & -0.0027 \\
\hline \hline
\end{tabular}


a)

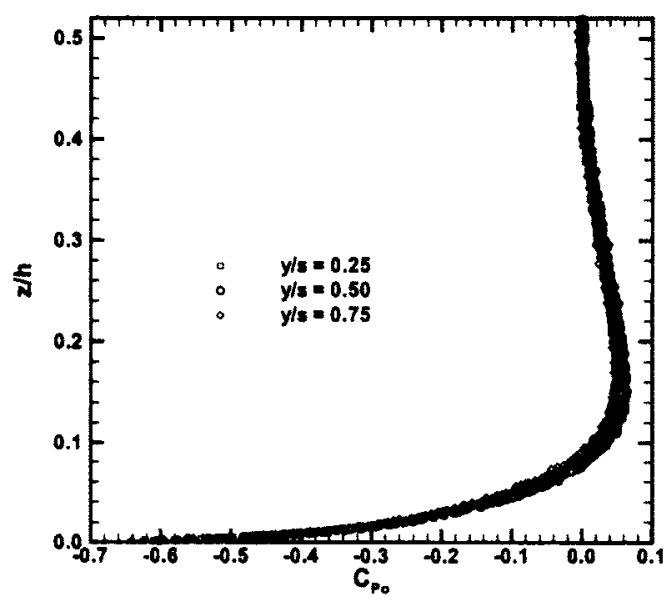

b)

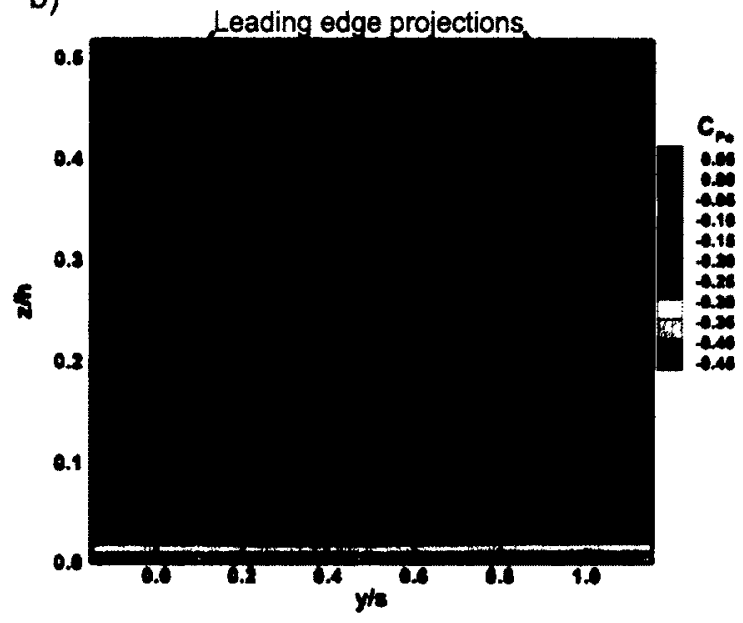

Figure 3.9: Total pressure deficit coefficient at inlet plane (a) Pitot-probe and (b) three-hole probe results

\subsubsection{Seven-Hole Probe Axial Traverses}

Detailed measurements of the flow field downstream of the cascade and within the blade passage were taken using the seven-hole pneumatic probe described in Section 3.9.3.

The first measurements were taken on PAKB. Data was taken on axial planes consisting of 976 data points within the blade passage. The exit conditions of the cascade were documented using 1288 measurement points at $140 \%$ axial chord downstream of the leading edge. Spanwise resolution of the measurement grids was held constant at $1.33 \%$ of span. The pitchwise resolution varied from $2.78 \%$ of pitch at $63 \%$ axial chord to $4.05 \%$ of pitch at the downstream measurement plane. The measurement resolution for PAKB is illustrated in Figures $3.10 \mathrm{a}$ and $3.10 \mathrm{~b}$ for the downstream and $0.8 B_{x}$ planes, respectively. These figures show the ability of the data to resolve the flow structures as demonstrated by the total pressure deficit coefficient $\left(C_{p o}\right)$.

When taking measurements for the more highly-loaded PAKD airfoils it was realized that the downstream intervals described above were inadequate for properly resolving the 
a)

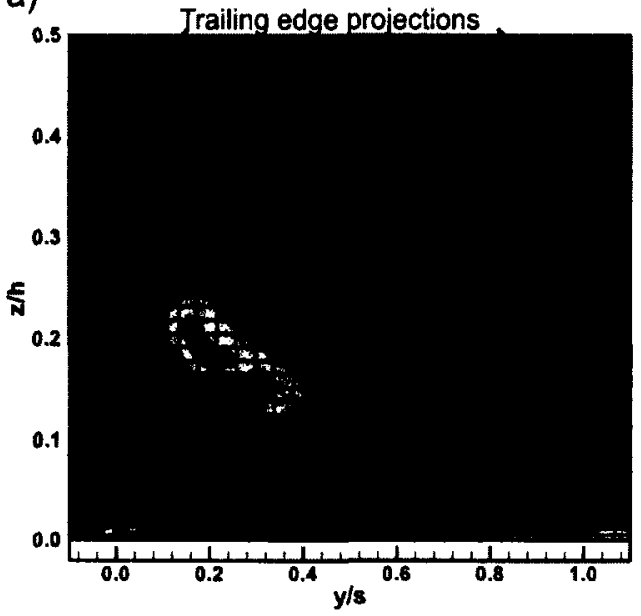

b)

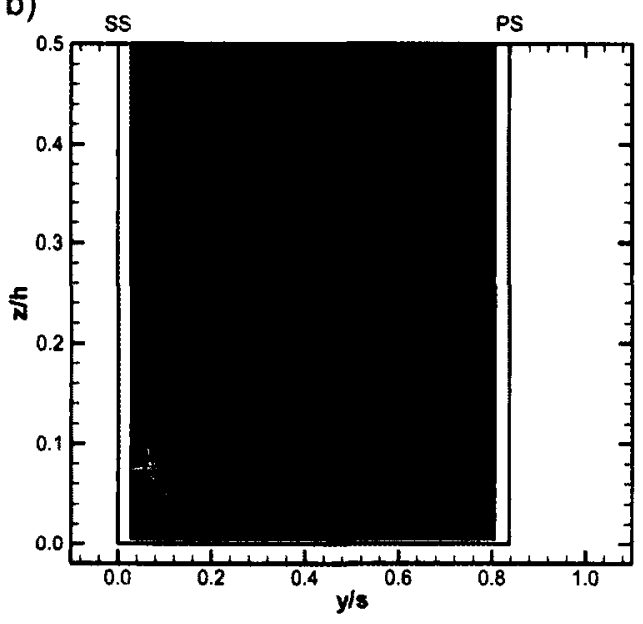

c)

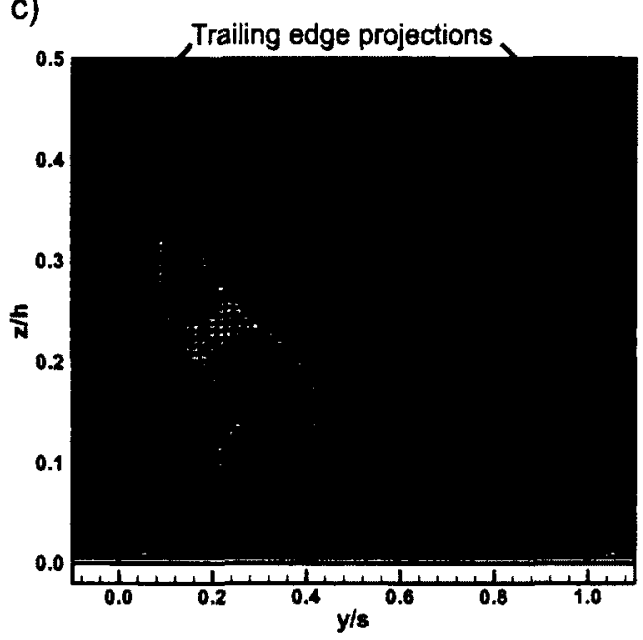

d)

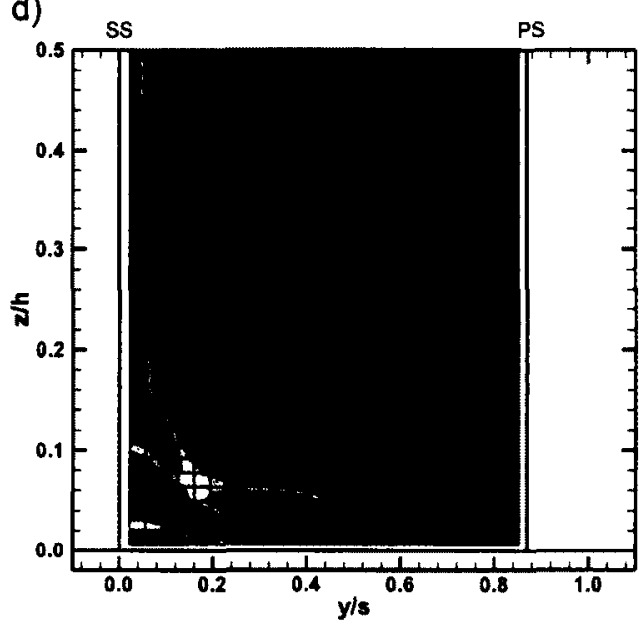

Figure 3.10: Contours of total pressure deficit coefficient overlaid with measurement intervals for PAKB (a \& b) and PAKD-F (c \& d)

two-dimensional wake, and therefore the profile losses for these cascades. As a result the interval density was doubled for all subsequent downstream measurements. These planes then consisted of 5038 data points. The resolution of the downstream measurement grids were $0.75 \%$ of span in the spanwise direction $1.81 \%$ of pitch in the pitchwise direction. Since the data from within the blade passage did not capture the suction and pressure surface boundary layers, they were not used to quantify the loss. These measurements were used primarily to gain insights into the development of the secondary flow. The intra-passage 
measurements were taken with a resolution on the order of that described above for PAKB. The number of data points for these planes varied from 1150 to 1334 . The measurement resolution for PAKD-F is demonstrated in Figures 3.10c and 3.10d for the downstream and $0.8 B_{x}$ planes, respectively.

All downstream and intra-passage measurements were sampled for 15 seconds at $1 \mathrm{kHz}$.

\subsubsection{Oil Surface Flow Visualization}

Oil surface flow visualizations were conducted on the blade suction surface and turntable endwall of the test section (the same wall where probe measurements were taken). Oil mixtures with different viscosities were used to create multiple visualizations which led to the interpreted streamlines. The PAKB flow visualizations were carried out using titanium dioxide pigment. For the PAKD and PAKF test cases, lamp black pigment was used in the blade surface mixtures and titanium dioxide was used for the turntable endwall. The opposing wall of the test section is removable and made of Plexiglas to allow physical and visual access.

During the preparation of the pigment/oil mixture care was taken to ensure that the pigment was thoroughly blended. Mixtures were applied to the hardware sparingly, in as thin a layer as possible to reduce pooling and to minimize the effect on the surface flow. 


\section{Chapter 4}

\section{Description of Computational}

\section{Method}

\subsection{Introduction}

This section outlines the details of the computational fluid dynamics (CFD) simulations. The chapter begins with a summary of the numerical setup. The grid independence study is then presented with some comparisons to experimental data for validation.

\subsection{Numerical Setup}

The simulations were performed using the ANSYS-CFX commercial software package (versions 5.7.1, 10.0, and 11.0). The Shear-Stress-Transport (SST) two-equation turbulence closure model (Menter, 1994) is applied to the Reynolds-averaged Navier-Stokes (RANS) equations. The energy equation was not solved as there are no significant compressibility effects or sources of heat transfer in the experiment. The conservation of mass and 
momentum equations are discretized to second-order accuracy by central differencing. First-order upwind discretization was applied to the turbulence closure equations by recommendation of the solver manual to avoid robustness issues. The results of those simulations were used as an initial result for a subsequent simulation where the High Resolution discretization scheme was applied to the turbulence closure equations. The application of the pseudo-second-order High Resolution scheme had negligible impact on the predicted pressure loss performance or flow angularity. Consequently it was deemed unnecessary to pursue the second round of simulations implementing the High Resolution scheme for the turbulence closure equations.

The algebraic multigrid scheme was enabled for all simulations performed. The multigrid approach hastens the reduction of errors having longer wavelengths (typically on the order of the domain size). These errors normally require many iterations to disappear, while shorter wavelength errors (on the order of grid spacing) are reduced relatively quickly. The algebraic multigrid creates a series of coarser meshes by summing the discretized fine mesh equations during the course of iterations, then refines the mesh to produce an accurate final solution. There are several criteria for determining how the fine mesh equations are summed. The method implemented in this work determines the summation of equations based on gradients in the hydrodynamic equations. This option was implemented in version 11 of the software and offered better convergence rates relative to the previous default criteria that summed equations based on gradients in pressure. With the algebraic multigrid disabled it was very difficult, if not impossible to achieve convergence. It was found that long wavelength pressure waves persisted in the domain and hindered the convergence process.

Once a converged solution was reached with the numerical configuration outlined above the Gamma-Theta transition model (Menter et al., 2004) was implemented in order to predict boundary layer transition to turbulent flow. This final step was essential as a fully turbulent solution will not predict loss and blockage correctly. Unless otherwise stated the 
results presented have the transition model applied. The implementation of the transition model typically resulted in lower predicted profile losses, and generally better agreement with experimental results.

\subsection{Grid Independence Study}

Grid independence studies were performed in two phases, first on a two-dimensional sliver of the cascade at midspan, and then on a three-dimensional half-span domain. The two-dimensional study is discussed first.

\subsubsection{Two-Dimensional Domain}

The two-dimensional grid independence study was carried out using the PAKF blade profile. This cascade has the largest pitch spacing, and it was reasoned that the node density sensitivity would be applicable to the other cascades used in the study. The twodimensional domain is shown in Figure 4.1. The domain extends $1.2 B_{x}$ upstream and 2 $B_{x}$ downstream of the leading edge, where $B_{x}$ is the axial chord length. One element spans the $1.00 \mathrm{~mm}$ thickness, compared with the $75 \mathrm{~mm}$ axial chord. As such, the w-momentum equation was not solved for the $2 \mathrm{D}$ cases reducing the computational effort in the study. The domain was created as a CAD model in Pro/E and linked to the ICEM meshing software via the ANSYS Workbench. Care was taken to ensure that the domain was translational periodic in the pitchwise direction. This was done by creating a piecewise-continuous spline for the pressure side periodic boundary, exporting the coordinates of the spline to an ASCII formatted file, then re-importing the coordinates into a new coordinate system that was offset by the blade pitch.

The blade pressure and suction surfaces were split at the geometric leading and trailing 


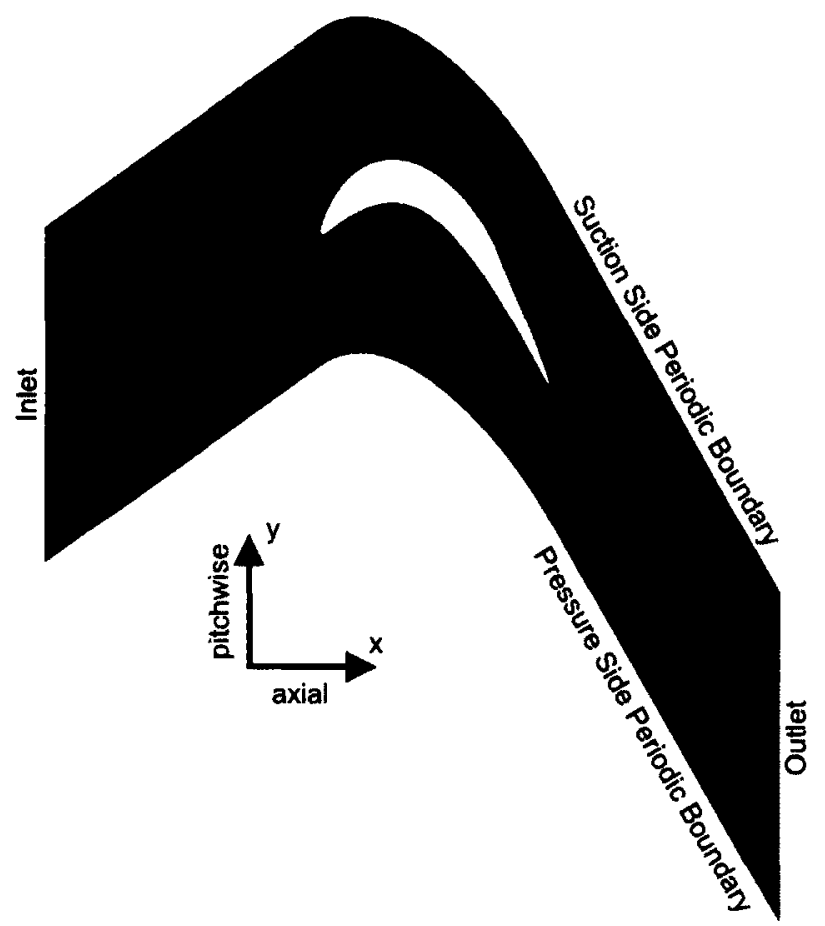

\section{Figure 4.1: CFD Domain}

edges. That is, the surfaces were split where the bisector of the wedge angle meets the blade surface. This was done so that the loading predictions could be easily plotted. The blade coordinates were ordered clockwise from the trailing edge around the pressure surface to the leading edge, and back around the suction surface to the trailing edge. The pressure-surface and suction-surface splines were set tangent to one another at the leading and trailing edges.

\subsubsection{Two-Dimensional Simulation and Boundary Conditions}

Air at $25^{\circ} \mathrm{C}$ is used as the working fluid and the density is assumed constant throughout the domain. The domain reference pressure, which is the datum from which all other pressures are taken was set to 1 atm absolute. This choice of reference pressure is typical for low-speed simulations near atmospheric conditions where variations in dynamic pressure are relatively small. For example, the dynamic pressure in the domain typically varies from 
$0-2500 \mathrm{~Pa}$. This variation is small relative to standard atmospheric pressure $(101325 \mathrm{~Pa})$; thus, calculations would be prone to numerical round-off error if an absolute pressure of 0 atm was set as the reference.

The pressure-side and suction-side periodic boundaries are set translational periodic to one another. A no-slip wall boundary condition was applied to the surfaces of the blade. The $x-y$ planes that make up the sides of the domain are set as symmetry planes. An average relative static pressure of $0 \mathrm{~Pa}$ was specified at the outlet boundary.

The inlet velocity was set to match experimental operating conditions (design incidence, Reynolds number of 126,000 based on inlet velocity and axial chord). Several iterations were used to determine appropriate values for the inlet turbulence quantities. It was found that an inlet turbulence intensity of $4.5 \%$ with a length scale of $0.025 \mathrm{~m}$ gave reasonable values of turbulence kinetic energy and turbulence decay throughout the domain.

\subsubsection{Two-Dimensional Grid Generation}

Grids were generated with ICEM versions 10.0 and 11.0. The domain was blocked with ten hex volumes and an O-grid surrounding the blade. The blocking is shown schematically in Figure 4.2. Selected results will be presented for seven two-dimensional domain grids of varying node density. The details of the grids are presented in Table 4.1. Letters " $a$ " through " $h$ " are used to designate the various edges of the blocking and the values in the table represent the number of nodes along those edges. " $h-h 1$ " designates the height of the first node from the surface of the blade in $\mathrm{mm}$ and " $\mathrm{h}$ ratio" denotes the expansion ratio away from the surface. The total number of nodes used in each domain is also tabulated. On occasion it was required to split "f" into several sections in order to ensure grid orthogonality at the blade surface.

Grids "G01" through "G04" represent increasing node density to a point where it is 
believed a grid independent solution has been reached. Grids "G05" through "G07" verify the fidelity of the solution produced by "G04" by increasing the node spacing along " $\mathrm{f}$ ", "g", and " $h$ " independently. The resolution changes along "f" and " $h$ " were intended to verify the prediction of the blade surface boundary layers by increasing the chordwise and blade normal node density. Increasing the resolution along "g" verified the prediction of the diffusion of the wake.

All grids were generated following the recommended practices outlined in the ANSYSCFX (2006) user manual under the heading "Mesh Visualization Advice" to assist convergence and reduce numerical inaccuracy. The recommended practices are as follows:

- Ratio of the longest edge length of an element to the shortest edge length $<100$

- Minimum face angle $>10^{\circ}$

- Ratio of volume for neighboring elements $<5$

- Number of elements connected to each node $<24$

- Edge expansion ratio of neighboring elements $<1.3$

- Near wall spacing such that first node $y^{+}<2$ and expansion ratio $\cong 1.1$

Table 4.1: Two-dimensional grid sensitivity parameters

\begin{tabular}{cccccccccccr} 
Grid & a & b & c & d & e & f & g & h & h - h1 & h ratio & Nodes \\
\hline \hline G01 & 11 & 5 & 5 & 14 & 26 & 40 & 25 & 15 & 0.05 & 1.23 & 7,800 \\
G02 & 11 & 6 & 10 & 14 & 26 & 50 & 25 & 20 & 0.005 & 1.31 & 11,000 \\
G03 & 20 & 9 & 20 & 20 & 26 & 115 & 40 & 27 & 0.0015 & 1.27 & 32,000 \\
G04 & 26 & 12 & 20 & 26 & 34 & 156 & 52 & 35 & 0.0015 & 1.18 & 54,000 \\
G05 & 26 & 12 & 20 & 26 & 34 & 196 & 52 & 37 & 0.0015 & 1.17 & 66,000 \\
G06 & 26 & 12 & 20 & 26 & 34 & 156 & 85 & 35 & 0.0015 & 1.18 & 60,000 \\
G07 & 26 & 12 & 20 & 26 & 34 & 156 & 52 & 70 & 0.0015 & 1.07 & 80,000 \\
\hline \hline
\end{tabular}




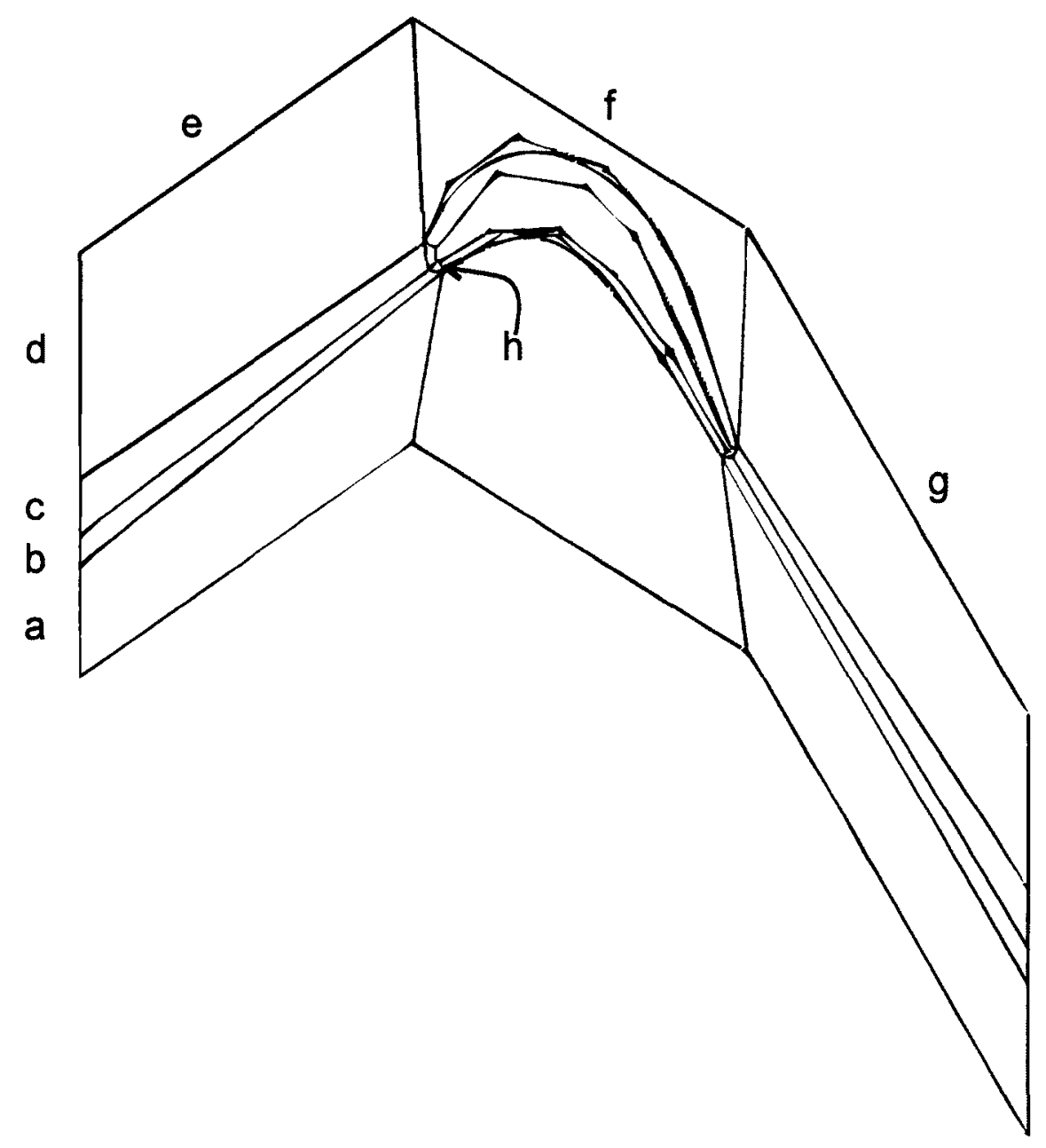

Figure 4.2: Schematic representation of blocking

\subsubsection{Two-Dimensional Study Results}

It was necessary to determine the relationship between the convergence of equation residuals and quantitative predictions prior to performing any detailed analysis of the CFD results. Figure 4.3 shows the predicted mass-averaged loss coefficient $\left(Y^{\prime}\right)$ and maximum equation residuals plotted as a function of solution accumulated time step. These results are typical for the simulations performed throughout the computational study. The solution began with the default domain initialization. The last of the equation residuals have reached 
their asymptote by about 230 time steps and there is negligible change in $Y^{\prime}$ after about 170 time steps. In accordance with these results, all simulations were run to 350 time steps with max equation residuals less than $10^{-5}$ to ensure convergence.

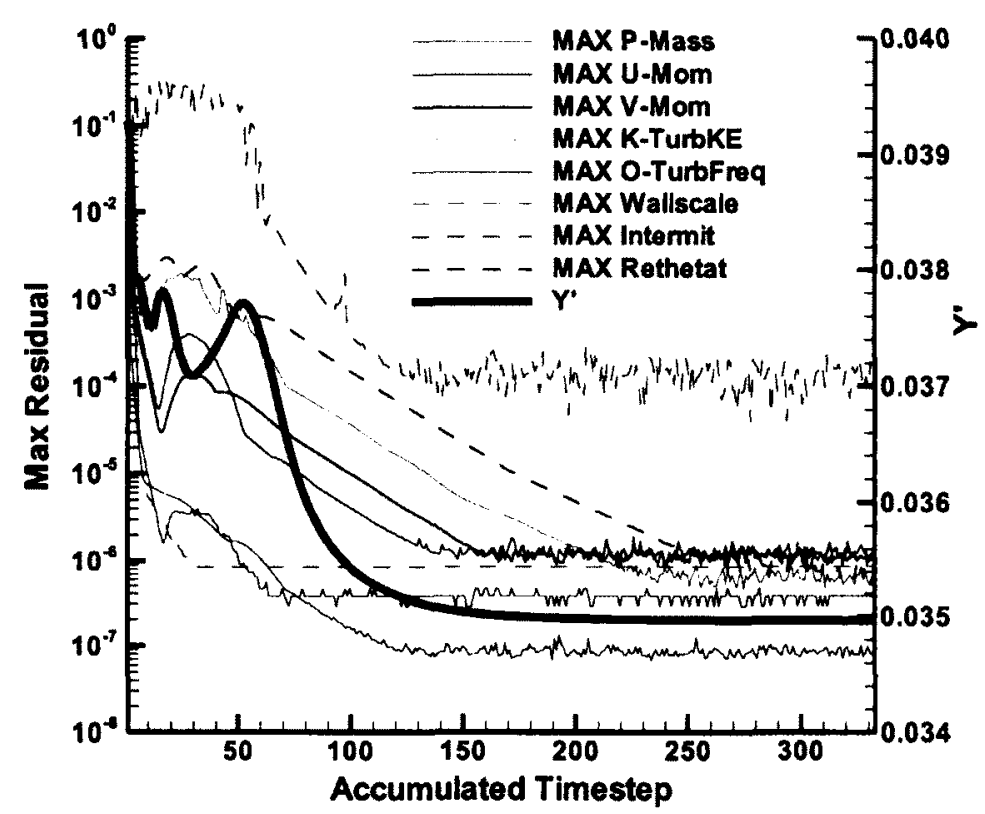

Figure 4.3: Relationship between equation residuals and predicted loss coefficient

Figure 4.4 shows mass-averaged loss coefficient $\left(Y^{\prime}\right)$ predicted with grids "G01" through "G07" as well as those measured experimentally with the seven-hole pressure probe (7HP) at $1.20 B_{x}, 1.40 B_{x}$ and $1.66 B_{x}$. Similarly, the predicted and measured mass-averaged flow deviation angle $\left(\delta^{\prime}\right)$ is presented in Figure 4.5. Both Figures 4.4 and 4.5 demonstrate that grid independence achieved by grid "G04", and that no further benefit is realized by increasing the node count along block edges "f", "g", and " $h$ ".

The results of Figure 4.5 show that predicted flow angles are within the experimental uncertainty of measured values for grid "G04". Figure 4.4 shows reasonably good agreement between predicted and measured values of loss coefficient as well, albeit differences are 


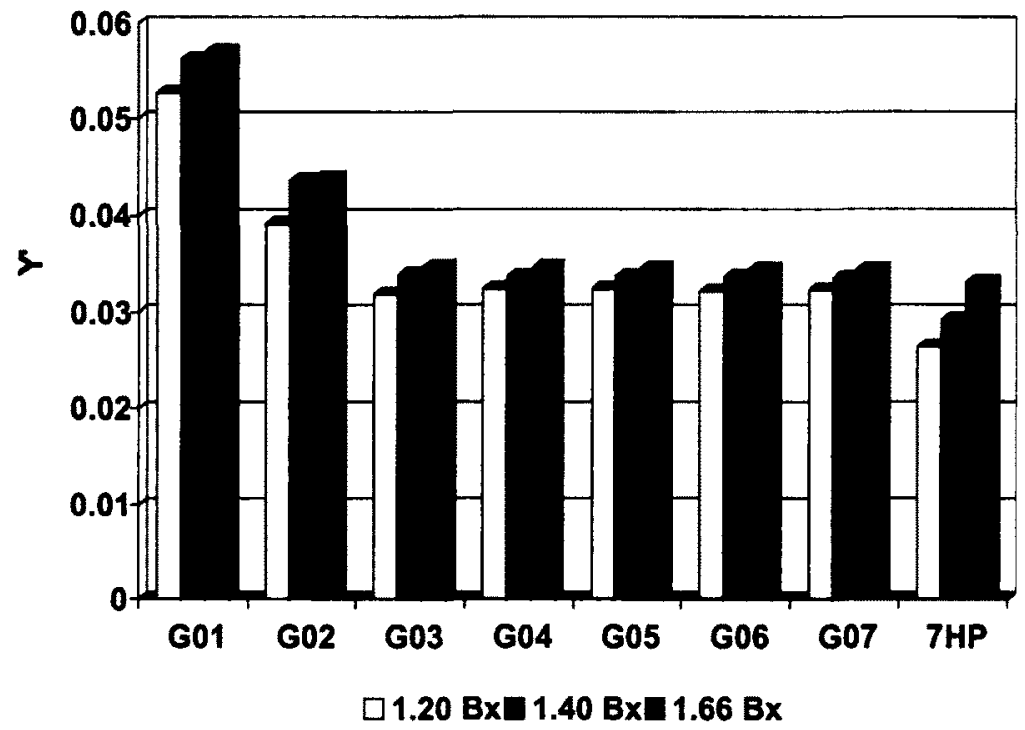

Figure 4.4: Predicted and measured mass-averaged loss

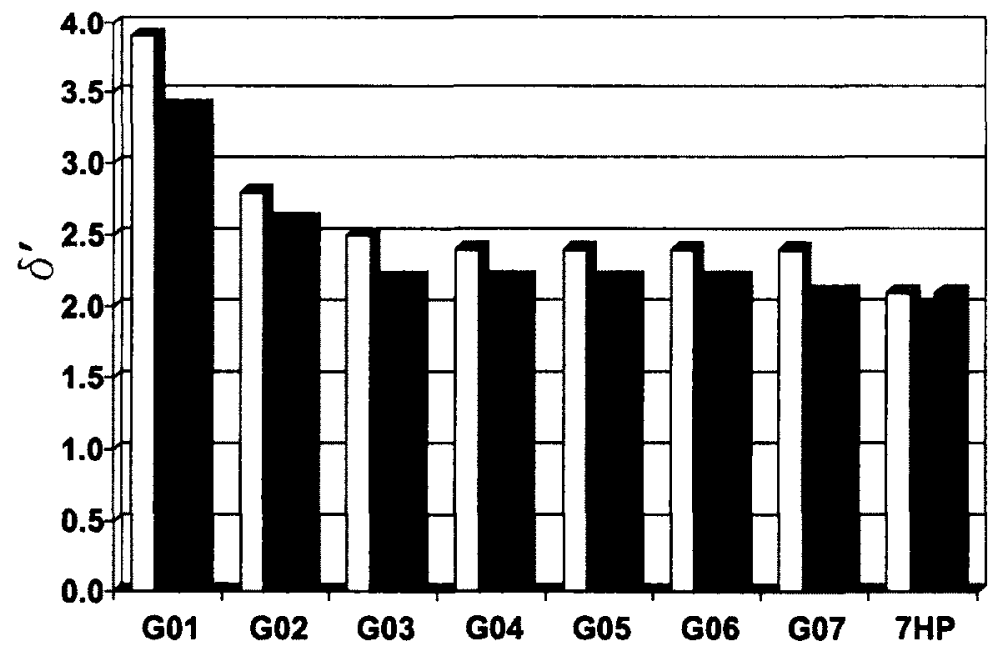

$\square 1.20 \mathrm{Bx} \square 1.40 \mathrm{Bx} \square 1.66 \mathrm{BX}$

Figure 4.5: Predicted and measured mass-averaged flow deviation

well outside the experimental uncertainty. The agreement seems to improve with distance downstream from the blade row. This trend is examined in Figures 4.6, 4.7 and 4.8, which plot the total pressure deficit coefficient $\left(C_{P_{0}}\right)$ through the wake at $1.20 B_{x}, 1.40 B_{x}$ and $1.66 B_{x}$ downstream, respectively. 


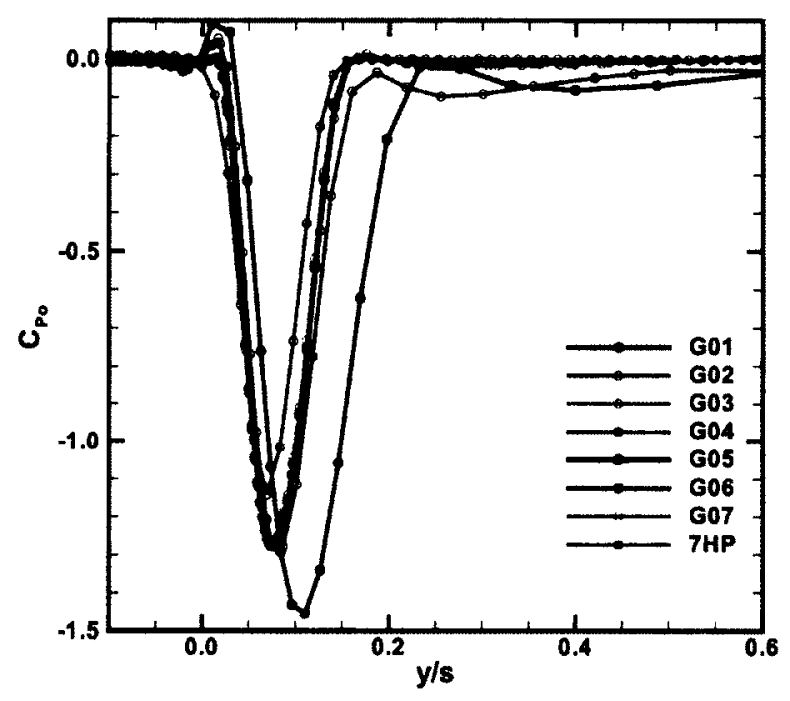

Figure 4.6: Midspan wake, predicted and measured total pressure deficit coefficient at $1.20 B_{x}$

Figure 4.6 shows the measured and predicted values of $C_{P o}$ through the wake at $1.20 B_{x}$. The results of grids "G01" and "G02" clearly show that a grid independent solution is not achieved at those node counts. The results of grids "G03" through "G07" are essentially indistinguishable from one another and accurately capture the width of the wake. However, they considerably over predict the total pressure deficit.

The measured and predicted wakes at the $1.40 B_{x}$ plane are shown in Figure 4.7. Once again grids "G03" through "G07" produce coincident solutions that over predict the total pressure deficit in the wake. The computations appear to under predict the diffusion, and the measured wake width is larger than predicted.

The results at $1.66 B_{x}$ (Figure 4.8) continue to exhibit the trends developed by $1.40 B_{x}$. Once more, the total pressure deficit is over predicted, and the diffusion is under predicted. In Figure 4.4 it was seen that the agreement between measured and predicted mass-averaged loss coefficient was better further downstream of the blade row. After inspection of the total 


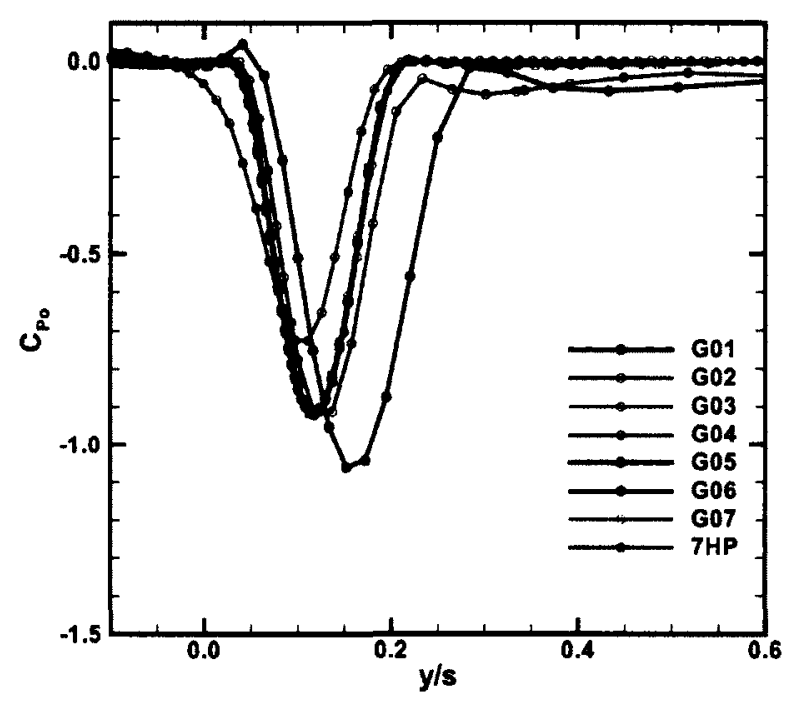

Figure 4.7: Midspan wake, predicted and measured total pressure deficit coefficient at $1.40 B_{x}$

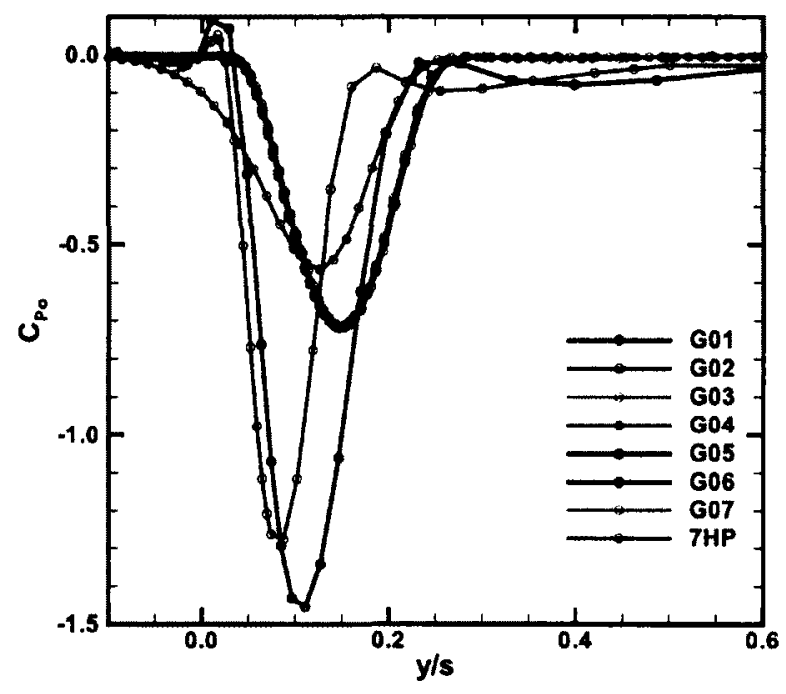

Figure 4.8: Midspan wake, predicted and measured total pressure deficit coefficient at $1.66 B_{x}$ 
pressure deficit coefficient through the wake in Figures 4.6 through 4.8 , it is evident that this trend is a result of a trade-off between an over prediction of loss and an under prediction of diffusion.

The predicted loading distribution is plotted for selected grids in Figure 4.9. Again, "G04" produces a grid independent solution, and there is no value added by increasing node count along the blade surface (length " $f$ ", "G05") or in the wall normal direction (length " $h$ ", "G07") since the three solutions are identical. A total of 21 static pressure tappings were used to measure the loading distribution, 15 of which were located along the suction surface. The predicted loading matches the measured results everywhere but the three taps downstream of the suction peak on the suction surface, where the discrepancy is believed to be a result of a separation bubble that was not captured by the two-dimensional simulations. This is discussed further in Chapter 8.

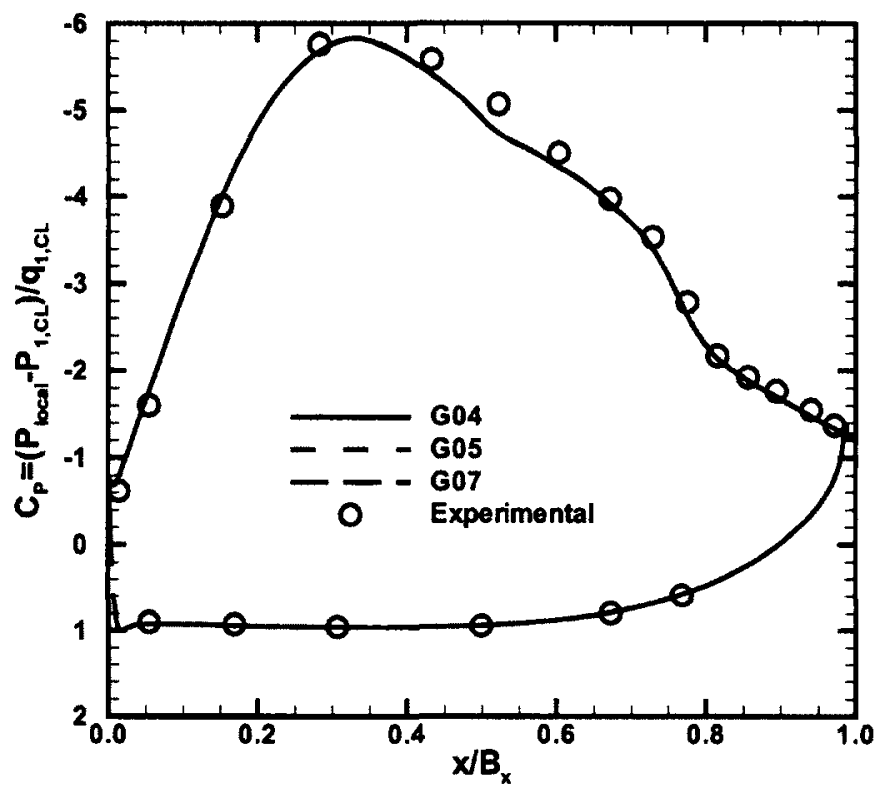

Figure 4.9: Predicted and measured midspan loading distribution

The effect of the grid on the predicted transition process can be seen be examining 
the skin friction along the suction surface, as presented in Figure 4.10. The skin friction coefficient is defined as $C_{f}=\tau_{w} / q_{1}^{\prime}$, where $\tau_{w}$ is the wall shear stress and $q_{1}^{\prime}$ is the massaveraged inlet dynamic pressure. As before, grids "G01" and "G02" produce erroneous solutions. Grid "G03" predicts a transition location $\left(0.48 B_{x}\right)$ slightly downstream of subsequent grids, and the location appears to move upstream with increasing grid density. Grid "G07" predicts the furthest upstream transition location and it's wall normal expansion ratio is less than 1.1. These results are in agreement with the ANSYS-CFX (2006) user manual which indicates that diminishing returns are obtained for expansion ratios less than 1.1 in the "ANSYS CFX Transition Model" subsection.

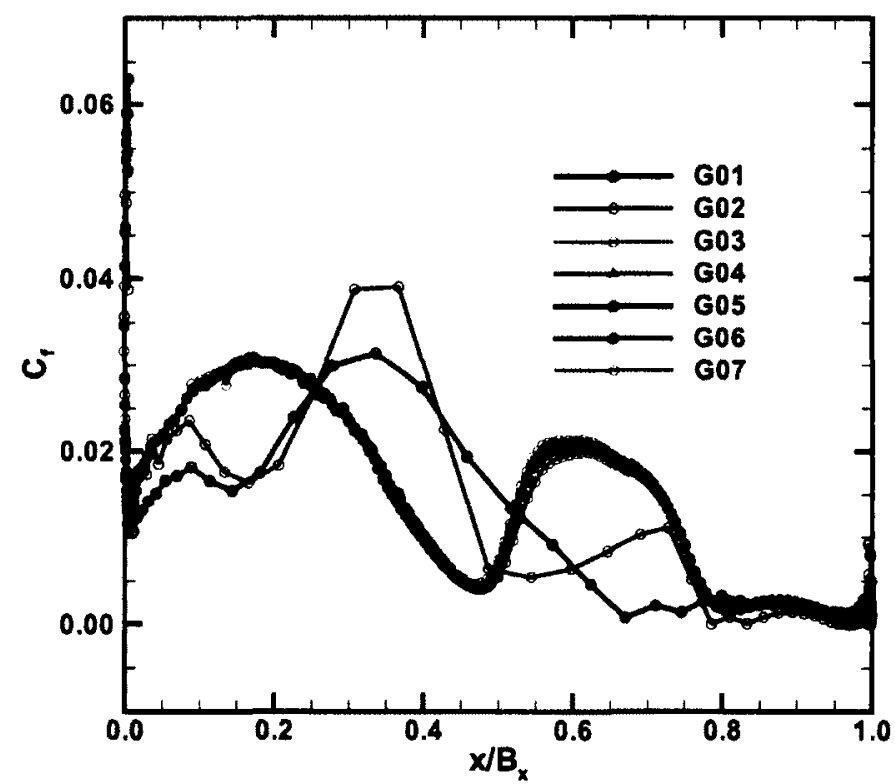

Figure 4.10: Predicted skin friction coefficient $\left(C_{f}\right)$ on suction surface

In summary, it can be said that grid "G04" produced a grid independent solution. Grids "G05" and "G07" independently increased the resolution along " $\mathrm{f}$ " and " $\mathrm{h}$ ". These simulations verified the prediction of the blade surface boundary layers by increasing the chordwise and wall normal node density. Grid "G06" confirmed the prediction of the 
diffusion of the wake by increasing the resolution along "g". The solutions from grids "G05" through "G07" offered negligible changes in predicted losses, exit flow angle, loading and suction surface skin friction coefficient. As such, the increased node count is unjustifiable.

\subsubsection{Three-Dimensional Domain}

The three-dimensional grid sensitivity was carried out using the PAKD-F planar endwall configuration as the 3D PAKF simulations predicted a stalled blade row. Further details on the stalled PAKF simulations are given in Chapter 8. The three-dimensional domain was configured as described in Section 4.3.1: the domain extends $1.2 B_{x}$ upstream and 2 $B_{x}$ downstream of the leading edge. Half the blade passage is modeled from the wall to midspan.

\subsubsection{Three-Dimensional Simulation and Boundary Conditions}

The simulation and boundary conditions for the three-dimensional computations were the same as those described in Section 4.3.2 except for the following:

The midspan plane was set as a symmetry plane; and a no-slip wall boundary condition was applied to the endwall.

Inlet boundary layer profiles were set for the axial $(u)$ and pitchwise $(v)$ components of velocity as well as the turbulent kinetic energy $(k)$ and the turbulent frequency $(\omega)$. The inlet boundary layer profiles are shown in Figures $4.11 \mathrm{a}$ and $4.11 \mathrm{~b}$. The spanwise component of velocity $(w)$ was set to zero throughout the inlet boundary layer. These profiles are identical to those used by PWA for the design of the contoured endwalls and post-test predictions. The boundary layer displacement thickness and momentum thickness have been matched to the conditions documented in the wind tunnel. The presence of the 
total pressure non-uniformity described in Section 3.10.3 has been omitted in the simulations based on the argument that it is convected through the cascade, as discussed in Section

\subsection{2.}
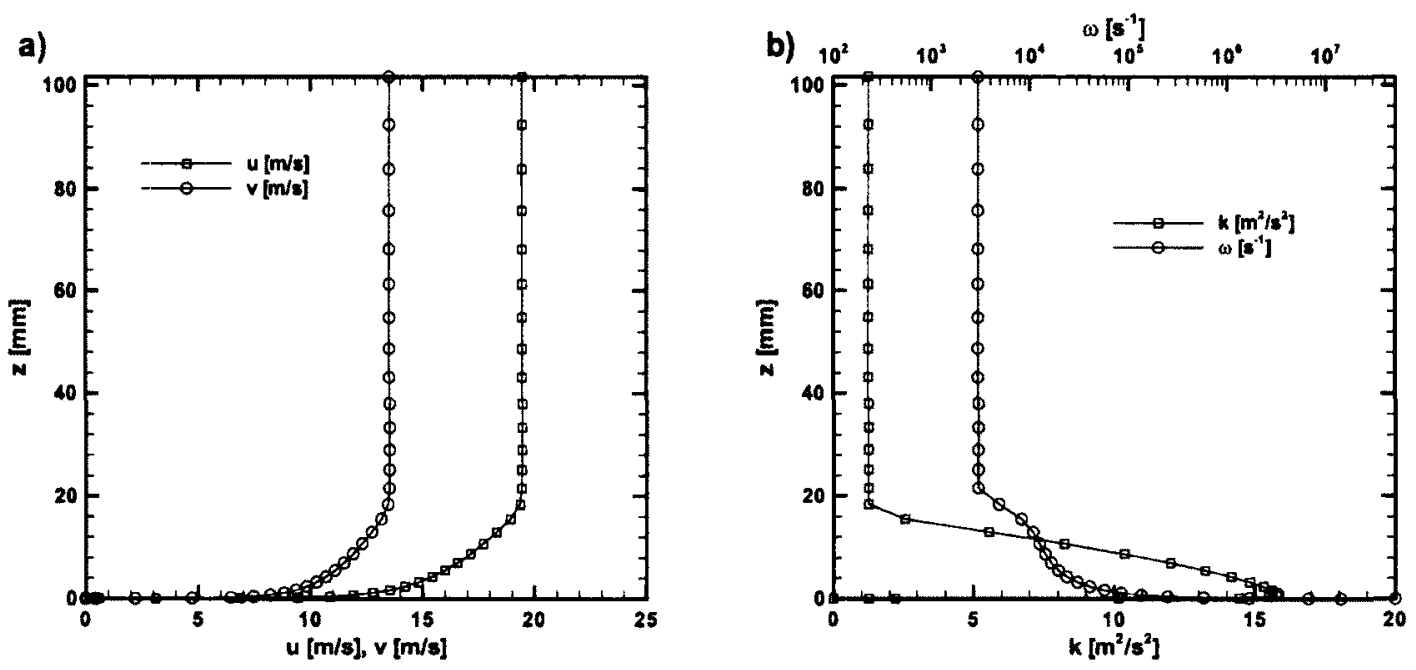

Figure 4.11: Inlet boundary layer profiles for (a) components of velocity and (b) turbulence quantities

\subsubsection{Three-Dimensional Grid Generation}

The domain was meshed using the blocking strategy and practices described in Section 4.3.3. A node distribution approximately equivalent to that of grid "G04" was employed on the $\mathrm{x}-\mathrm{y}$ plane. Three grids were generated for the three-dimensional grid independence study and their details are given in Table 4.2. A bi-geometric node distribution was used in the spanwise direction. The number of spanwise divisions was varied keeping $y^{+}<2$ at the wall and a using wall-normal expansion factor of approximately 1.1. At midspan, the spacing was set such that the expansion factor was also roughly 1.1 .

The following section presents the results of the three-dimensional grid independence study. It will be shown that the solution generated by grid "3D-G02" is independent 
Table 4.2: Three-dimensional grid sensitivity parameters

\begin{tabular}{lcr} 
Grid & Spanwise Divisions & Elements \\
\hline \hline 3D-G01 & 30 & 550,000 \\
3D-G02 & 45 & 840,000 \\
3D-G03 & 60 & $1,130,000$ \\
\hline \hline
\end{tabular}

of spanwise grid resolution as grids "3D-G02" and "3D-G03" produce nearly identical solutions.

\subsubsection{Three-dimensional Study Results}

Three-dimensional grid independence was determined by examining distributions and integrated values of several aerodynamic parameters. These parameters were the total pressure deficit coefficient $\left(C_{P o}\right)$, the streamwise vorticity coefficient $\left(C_{\omega_{s}}\right)$, the coefficient of secondary kinetic energy $\left(C_{S K E}\right)$ and pitchwise exit flow angle $\left(\alpha_{2}\right)$.

Figure 4.12 presents a colour flood contour plot of $C_{\omega_{s}}$ overlaid with line contours of $C_{P o}$ at $1.20 B_{x}$ as predicted by the three computational grids as well as those measured with the seven-hole pressure probe. The line contours of $C_{P o}$ have two regions of high total pressure loss being indicated by arrows displaying the peak magnitude there. As the spanwise grid resolution is increased from Figures $4.12 \mathrm{a}$ to $4.12 \mathrm{c}$ the peak magnitude predicted increases. In all three instances the peak loss is over-predicted relative to the experimental measurements of Figure 4.12d.

The flood plots of streamwise vorticity coefficient have two regions of fluid containing positive vorticity and one region of negative vorticity. The physics of the endwall flow structure were introduced in Section 2.2, and will be discussed in greater detail in the subsequent results chapters. For now the magnitude of vorticity predicted is of interest. In general, as the spanwise grid resolution is increased the magnitude of vorticity associated 
a)

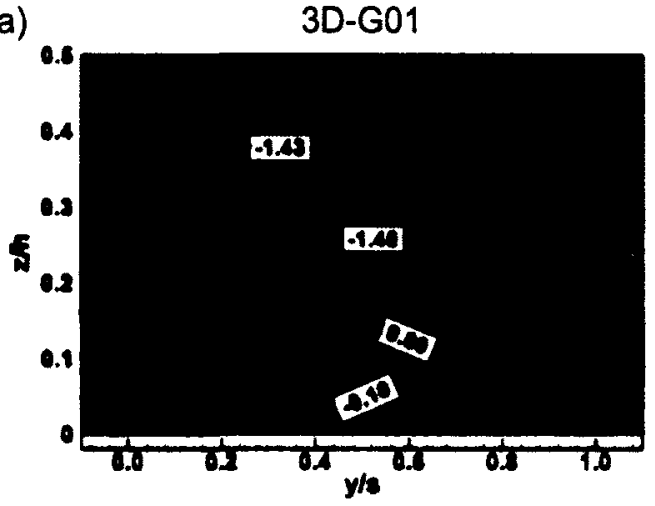

b)

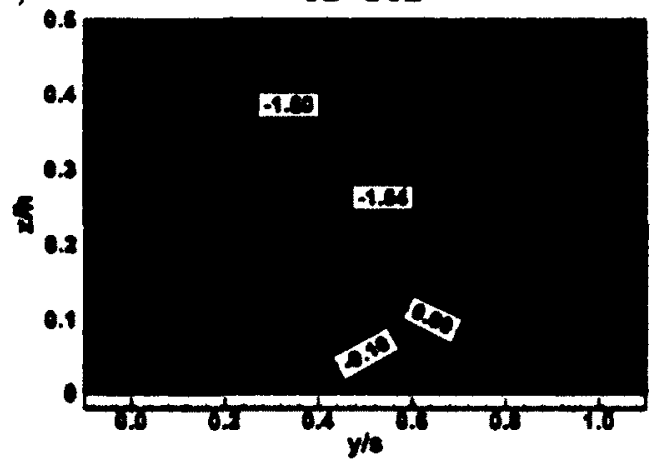

c)

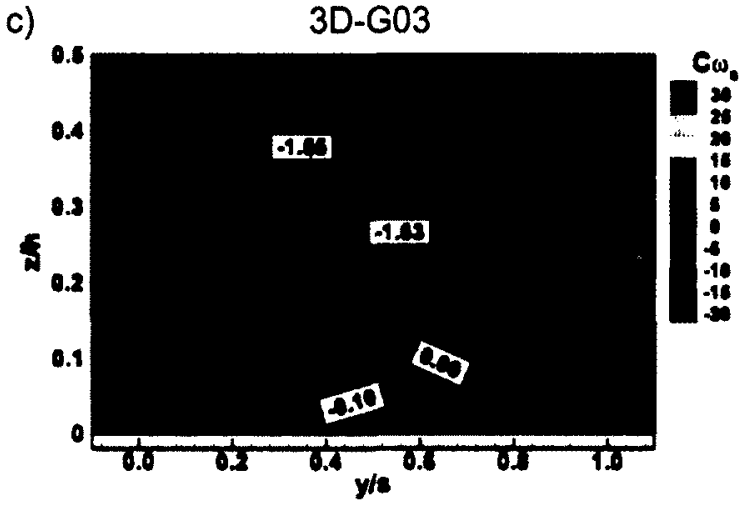

d)

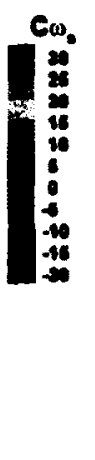

$7 \mathrm{HP}$

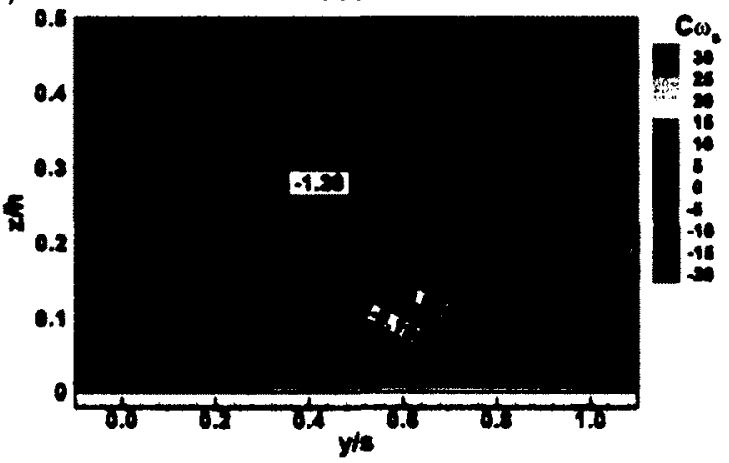

Figure 4.12: Predicted and measured flood of $C_{\omega_{s}}$ overlaid with line contours of $C_{P_{o}}$ at $1.20 B_{x}$ for grids (a) "3D-G01", (b) "3D-G02", (c) "3D-G03" and (d) 7HP

with the vortical structures increases slightly (on the order of several percent of the peak value). The simulations tend to over predict the magnitude of the positive vorticity field, and under predict the negative vorticity.

The results from the $1.20 B_{x}$ are pitchwise mass-averaged and presented in Figure 4.14. The subtle differences between simulations "3D-G02" and "3D-G03" are imperceptible; however, there is a noticeable difference from "3D-G01". The pitchwise mass-averaged $C_{P o}$ in Figure 4.14a show that increasing the spanwise node density offers improved resolution of the three-dimensional flow between $5 \%$ and $25 \%$ span. The influence of the resolution in this region is expected as the near-wall node distribution was fixed and the flow near midspan is two-dimensional and should be largely unaffected by spanwise spacing. Comparing the 
predicted results to those measured, the location of highest loss is predicted further from the endwall, toward midspan, and the maximum loss is over predicted. The overall penetration of the secondary flow toward midspan is also over predicted.

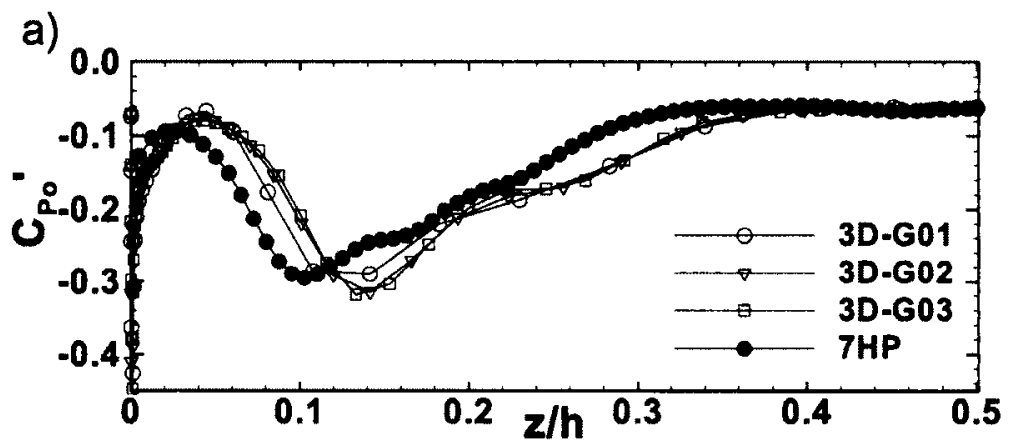

b)
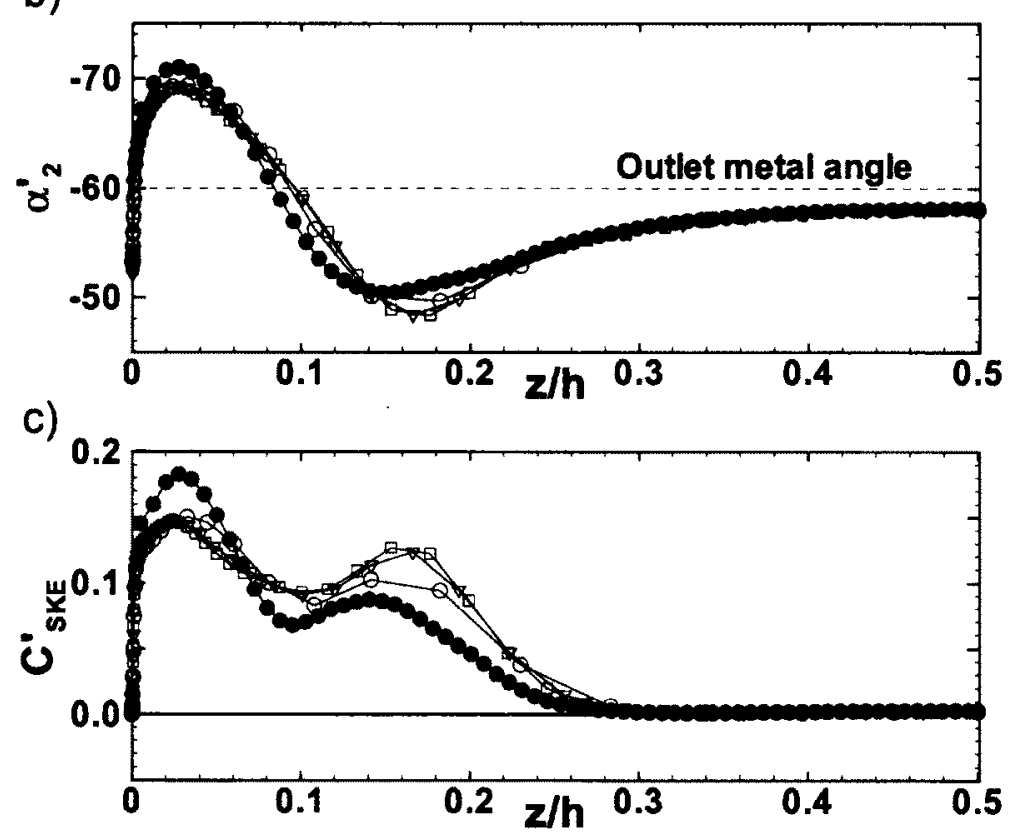

Figure 4.13: Predicted and measured mass-averaged (a) $C_{P o}$, (b) $\alpha_{2}$ and (c) $C_{S K E}$ at $1.20 B_{x}$

The pitchwise mass-averaged outlet flow angle, $\left(\alpha_{2}\right)$ is presented in Figure 4.14b. The predictions generated with the three grids are virtually indistinguishable. As such, the three simulations predict the $u$ and $v$ components of velocity independent of spanwise grid spacing. The simulations tend to over predict the pitchwise under-turning of the flow and the location of maximum under-turning was closer to midspan in comparison to the 
experimental measurements. The over-turning in the endwall region was under predicted.

The coefficient of secondary kinetic energy $\left(C_{\omega_{s}}\right)$ is pitchwise mass-averaged in Figure 4.14c. As above, "3D-G02" and "3D-G03" produce nearly identical solutions. Grid "3DG01' over predicts $C_{\omega_{s}}$ for $0.03<z / h<0.08$ and under predicts it for $0.08<z / h<0.24$. The coefficient of secondary kinetic energy scales on the sum of the squares of the $v$ and $w$ components of velocity. The differences seen with grid variation are a result of the dependence of the $w$ component of velocity on spanwise grid spacing.

Similar conclusions to those discussed above can be drawn for the predictions at the $1.40 B_{x}$ plane, except that the effects of spanwise node density are seen to a lesser extent. Floods of $C_{\omega_{s}}$ overlaid with line contours of $C_{P_{o}}$ at $1.40 B_{x}$ are presented in Figure 4.17. As before, the peak loss calculated shows a slight dependence on spanwise grid spacing (Figures 4.17a to $4.17 \mathrm{c}$ ); however, the effect is less than seen at $1.20 B_{x}$. The magnitude of the peak loss predicted is greater than that measured experimentally (Figure 4.17d).

The flood contours of streamwise vorticity coefficient exhibit similar trends to those discussed above as well. There is a slight dependence on spanwise grid density for the positive and negative vorticity where both increase by several percent as the node count is increased. Also as above, the positive vorticity is over predicted, while the negative vorticity is under predicted relative to the experimental measurements.

The results from the $1.40 B_{x}$ plane are pitchwise mass-averaged in Figure 4.15. Grids "3D-G02" and "3D-G03" generate similar solutions while "3D-G01" shows some dependence to spanwise node density from $0.06<z / h<0.24$. The grid independent solutions produce pitchwise mass-averaged results similar to those measured experimentally, except that the locations of the two loss peaks are further toward midspan. As above, the secondary flow is predicted to penetrate further into the passage $(0.20<z / h<0.38)$, and the region of two-dimensional flow at midspan has been reduced. 
a)

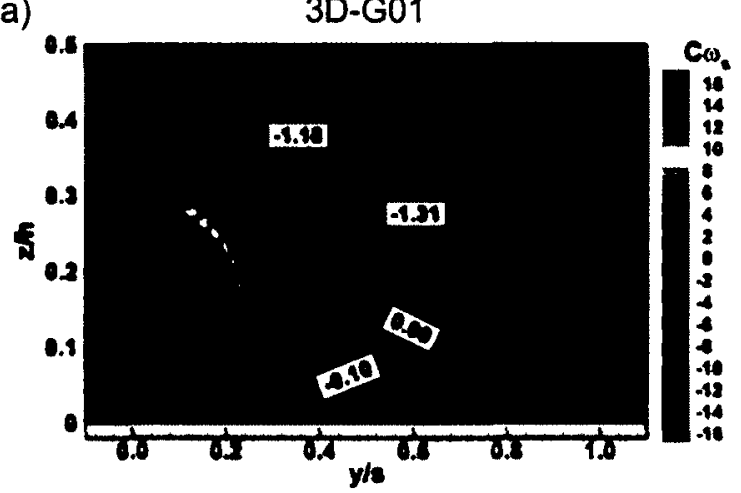

b)

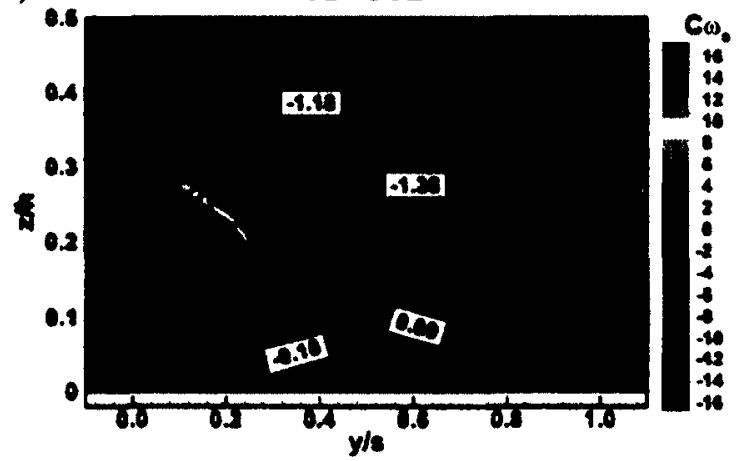

c)

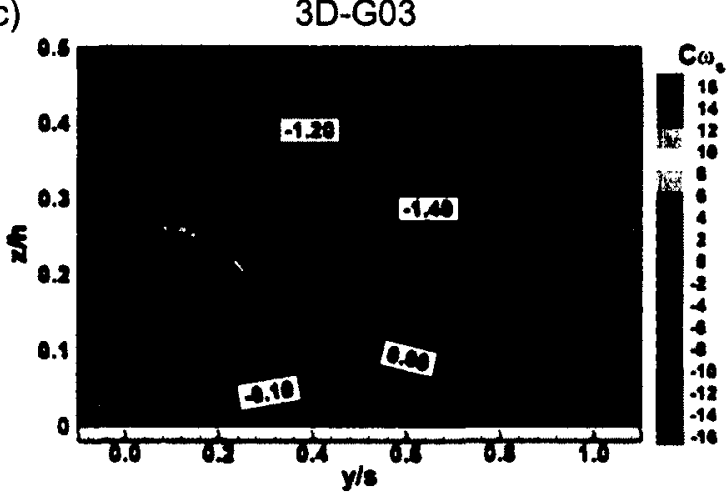

d)

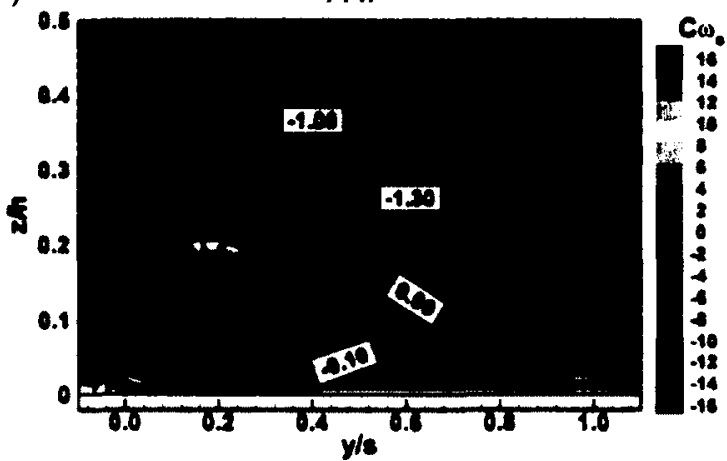

Figure 4.14: Predicted and measured flood of $C_{\omega_{s}}$ overlaid with line contours of $C_{P o} 1.40 B_{x}$ for grids (a) "3D-G01", (b) "3D-G02", (c) "3D-G03" and (d) 7HP

The pitchwise exit flow angle $\left(\alpha_{2}\right)$ results of Figure $4.15 \mathrm{~b}$ support the conclusion that grids "3D-G02" and "3D-G03" produce similar solutions. The underturning of the flow is over predicted by $2.1^{\circ}$ and the location of maximum underturning is $z / h=0.03$ closer to midspan relative to the experimental results.

The computed coefficient of secondary kinetic energy is compared to experiential results in Figure 4.15c. The predicted $C_{S K E}$ from grid "3D-G01" shows dependency on spanwise grid resolution as it did in Figure 4.13c. This is important to note since minimizing the secondary kinetic energy coefficient has been used as the optimization criterion for designing contoured endwalls (Yan et al., 1990 and Harvey et al., 2000). If a similar approach were to be attempted and the initial spanwise node distribution was too coarse prior to optimization, the perturbation of the endwall, and subsequent redistribution of nodes could erroneously 

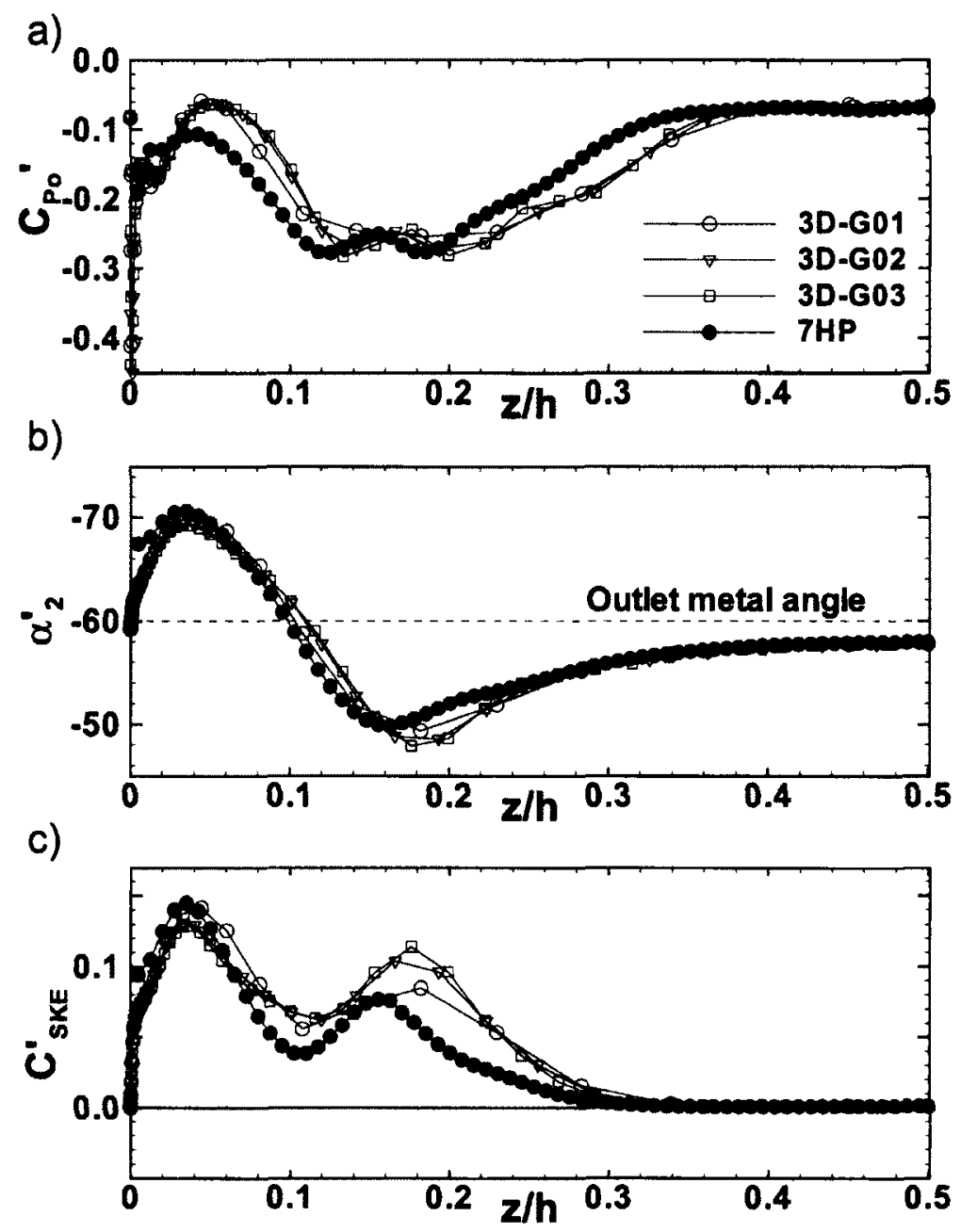

Figure 4.15: Predicted and measured mass-averaged (a) $C_{P o}$, (b) $\alpha_{2}$ and (c) $C_{S K E}$ at $1.40 B_{x}$

influence the result.

Figure 4.16 shows the mass-averaged total pressure loss coefficients $\left(Y_{\text {Total }}^{\prime \prime}\right)$ for the three computational grids and the experimental measurements. At $1.20 B_{x}$, the figure shows that as the spanwise grid resolution is increased, $Y_{\text {Total }}^{\prime \prime}$ tends to decrease further below the experimentally measured value. At $1.40 B_{x}$, some scatter exists in the predicted values of the loss coefficient. It should be noted that although these trends are visible, the variation is very small. The experimental measurement uncertainty for the seven-hole pressure probe 
is such that $C_{P_{o}}^{\prime \prime}$ is accurate to \pm 0.003 (from Section 3.9.3). The variation in computed $C_{P o}^{\prime \prime}$ is approximately one quarter of the probe uncertainty for the $1.20 B_{x}$ plane. At the $1.20 B_{x}$ plane, the computed variation is roughly one third of the probe measurement uncertainty.

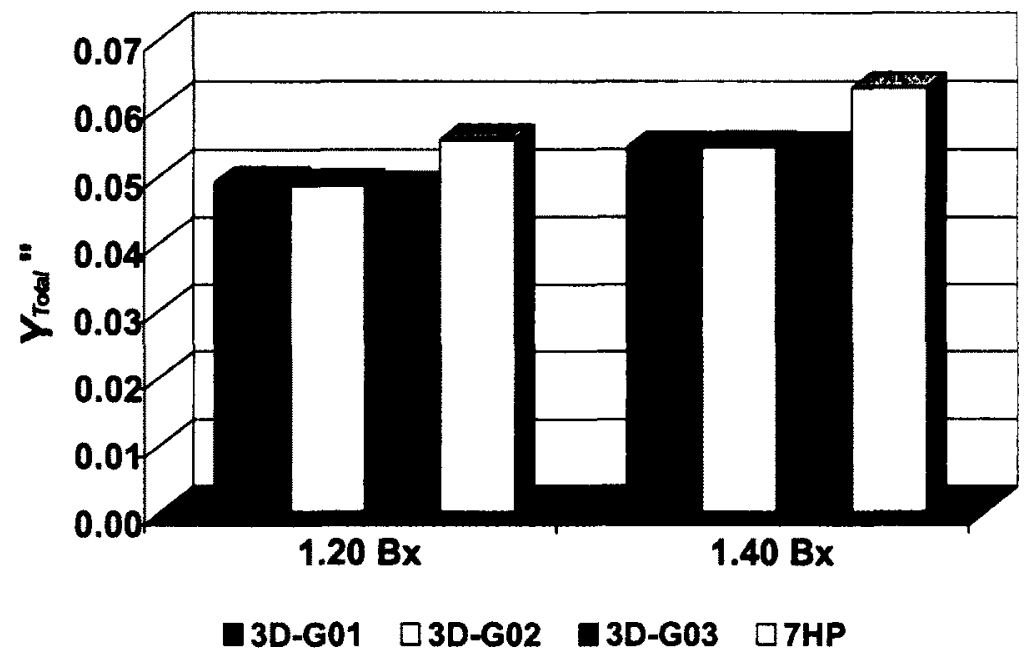

Figure 4.16: Predicted and measured mass-averaged $Y$ at $1.20 B_{x}$ and $1.40 B_{x}$

Of more interest is the fact that the computations tend to under predict the loss generated in turning the flow, and thus over estimate the performance of the blade row. This would be of concern to the turbine designer employing similar computational methods in the design process to predict efficiency. This thought will be elaborated on in subsequent chapters as computational results are compared to the experimental measurements.

In light of the results presented above it is concluded that the results of grid "3DG02" are essentially independent of node count. This computation grid consists of 840,000 elements. It can be noted that Praisner et al. (2007) found grid independence at approximately 800,000 elements for an $\mathrm{O}-\mathrm{H}$ topology grid using the PWA in-house solver. Also, Hjärne et al. (2007) claim grid independence at 690,000 elements for a linear cascade of turbine outlet guide vanes using the FLUENT solver. The spanwise node spacing of grid "3D-G03" was in fact implemented for three-dimensional half span meshes in this study since it offers little penalty in computation or post processing time relative to "3D-G02", 
yet it yields better resolution of the secondary flow structures.

The total pressure deficit coefficient predicted with "3D-G03" is compared to seven-hole pressure probe data in greater detail in Figure 4.17. Figure 4.17a shows a flood of predicted $C_{P o}$ overlaid with line contours of measured values to compare the size and location of the secondary flow structure. The secondary flow structure predicted is smaller than that measured, but the peak loss computed is higher. The locations of the loss peaks are predicted to be further away from the endwall than those measured.

a)

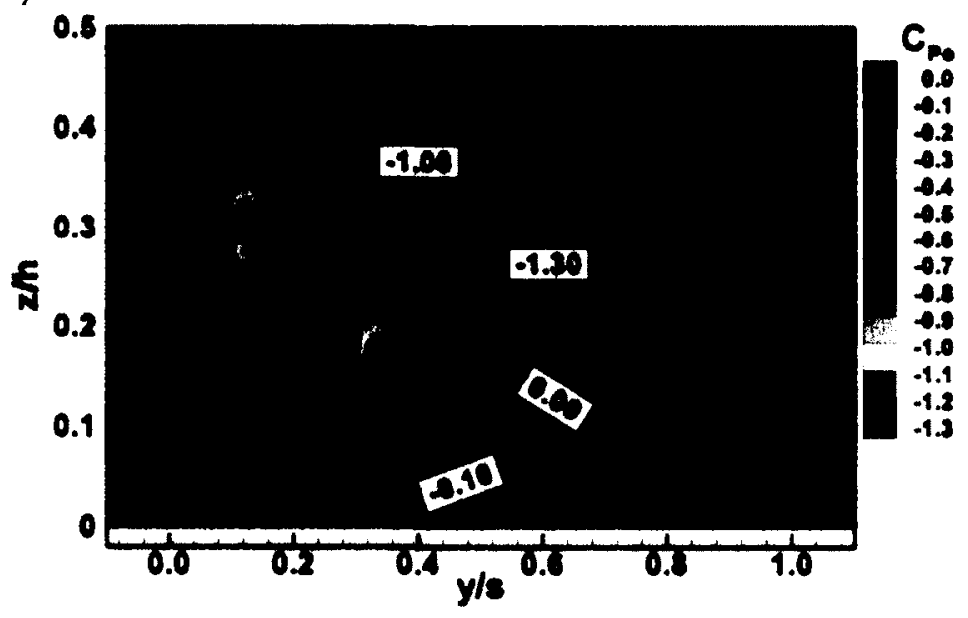

b)

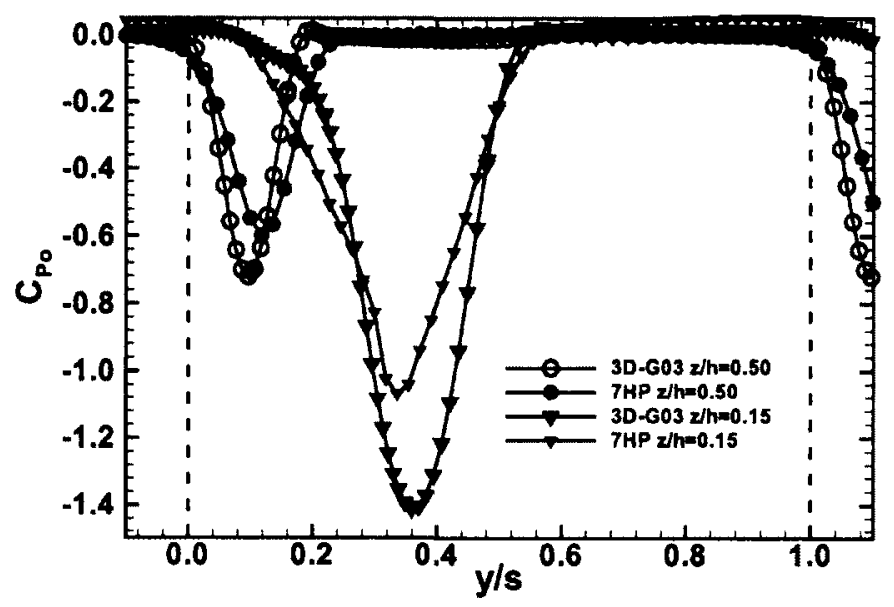

Figure 4.17: (a) Contour plot and (b) slice planes at $z / h=0.50$ and $z / h=0.15$ showing predicted and measured $C_{P_{0}}$ at $1.40 B_{x}$ 
Figure $4.17 \mathrm{~b}$ shows lines of $C_{P o}$ at constant spanwise distance from the endwall. At midspan $(z / h=0.50)$, the same trends outlined in Section 4.3 .4 are seen, namely that the simulations over predict the peak loss in the wake and under predict the diffusion with downstream distance from the trailing edge. The results at $z / h=0.15$ approximately bisect the peak loss region in the secondary flow predicted by the simulations. Once again the simulations over predict the peak magnitude of total pressure loss and under compute the diffusion of the secondary flow structure. 


\section{Chapter 5}

\section{Secondary Flow in a Turbine}

\section{Cascade with the Implementation}

\section{of Non-Axisymmetric Endwall}

\section{Contouring}

\subsection{Introduction}

This chapter examines the flow field of a linear cascade comprised of PAKB airfoils. This airfoil has been used extensively in low pressure turbine research. The cascade is first investigated here for the baseline case with a planar endwall. The results add intra-passage pressure and velocity measurements to the existing database.

Subsequently the cascade was tested with the application of non-axisymmetric endwall contouring. This chapter presents the baseline case in a study aimed at exploiting more 
highly loaded low-pressure turbine airfoils then that represented by PAKB. The focus of the work is on the physics of the endwall flow in order to enhance the understanding of the relationship between losses and secondary kinetic energy.

\subsection{Motivation}

The implementation of high-lift airfoils in low-pressure turbine design is an attractive means to reduce engine weight and manufacturing costs. Several studies (Hodson and Dominy, 1987a, Zorić et al., 2007a, Zorić et al., 2007b) have shown that increasing airfoil loading inherently leads to higher secondary losses. Thus it is desirable to manipulate the flow field in the endwall region to reduce secondary losses and potentially expand the design envelope.

Geometric modifications to the endwall region have been shown to yield lower secondary losses. Both Chung and Simon (1991) and Aunapu et al. (2000) studied the effects of endwall fences on the secondary flow in a linear cascade representation of a single turbine blade passage. Their investigations showed that the size and strength of the passage vortex could be reduced with the application of endwall fences. The improvements were thought to be a result of altering the trajectory of the passage vortex within the blade passage.

The use of non-axisymmetric endwall contouring within the blade passage for loss reduction has been studied by researchers in the past decade. Yan et al. (1990) and Harvey et al. (2000) presented methods for applying non-axisymmetric full-passage endwall contouring. The authors relied on predictions of cross-passage pressure gradient and secondary kinetic energy for design optimization based on the notion that simulations using a mixing-length turbulence model were unable to accurately predict loss.

Experimental assessments of endwall contouring were conducted by Hartland et al. 
(2000) and Rose et al. (2001). Both studies showed a marked reduction in losses as a result of the application of a profiled endwall. The improvement was attributed to a reduction in secondary kinetic energy and improved outlet flow quality based on reducing flow over/underturning in the pitchwise direction. Ingram et al. (2005) used the same design tool as Hartland et al. (2000) to develop a contoured endwall that was supposed to achieve maximum loss reduction. However, the computations were unable to predict a separation of the intra-passage boundary layer that was revealed by the experiment and that led to higher losses despite a reduction in blade row under/overturning and lower outlet secondary kinetic energy. The latter study demonstrates the ongoing need for experimental validation of computational results.

\subsection{Approach}

The current chapter examines the secondary flows of the low-pressure turbine cascade designated PAKB, with and without the implementation of endwall contouring. The focus is on understanding the physics of the endwall flow within the blade passage and downstream of the trailing edge and their effects on loss generation.

The blade row is shown in Figure 3.4 and the geometric details are given in Table 3.2. The testing was conducted at design incidence and an inlet Reynolds number of 126,000 base on axial chord.

\subsection{Endwall Contouring}

A rendering of the PAKB contoured endwall is presented in Figure 5.1 showing the three predominant geometric features. The contouring is comprised of a hill $(H)$ and two valleys $\left(V_{1}\right)$ and $\left(V_{2}\right)$ located within the blade passage. The maximum positive and negative 
amplitudes of the contoured endwall are $7.1 \%$ and $4.7 \%$ of axial chord, respectively. The effect of these geometric features on the flow field relative to the planar endwall will be discussed in regards to the flow visualizations and pressure probe measurements.

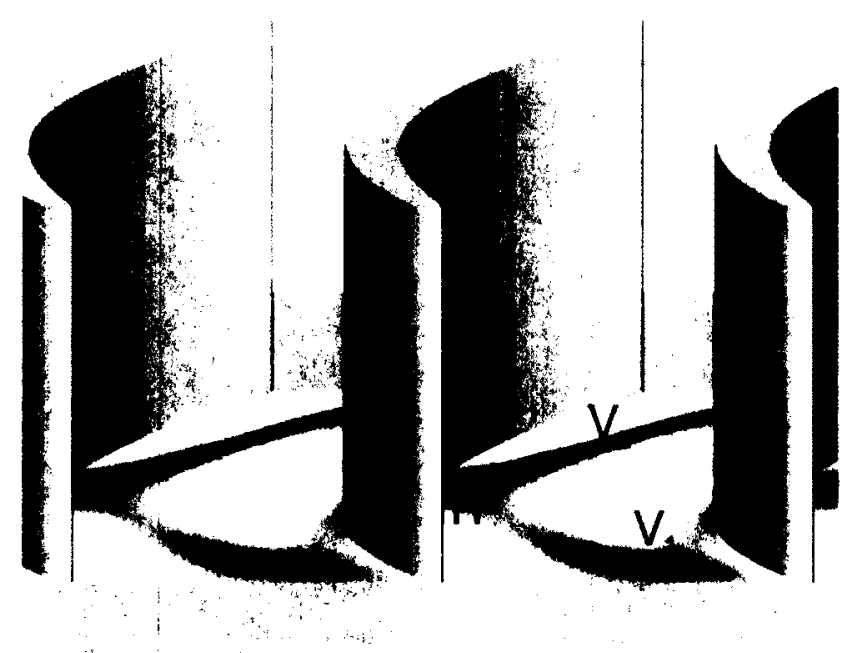

Figure 5.1: Isometric view of PAKB profiled endwall

\subsection{Experimental Results}

\subsubsection{Cascade Inlet Conditions}

The turbulence generating grid used in the present study caused excess total pressure loss near midspan resulting in a non-uniform freestream profile. The total pressure nonuniformity is seen in Figure 5.2 measured by a boundary layer traverse using a pitot probe $1.2 B_{x}$ upstream of the leading edge of the cascade at $y / s=0.5$. As was discussed in Section 3.10.3, the inlet non-uniformity was documented by traversing the inlet with a three-hole pressure probe over one blade-pitch from the wall to half span; the total pressure distribution was then mass-averaged to obtain the reference inlet total pressure coefficient $\left(C_{P_{0,1}}^{\prime \prime}\right)$. The mass-averaged total pressure coefficient and the inlet boundary layer parameters are 


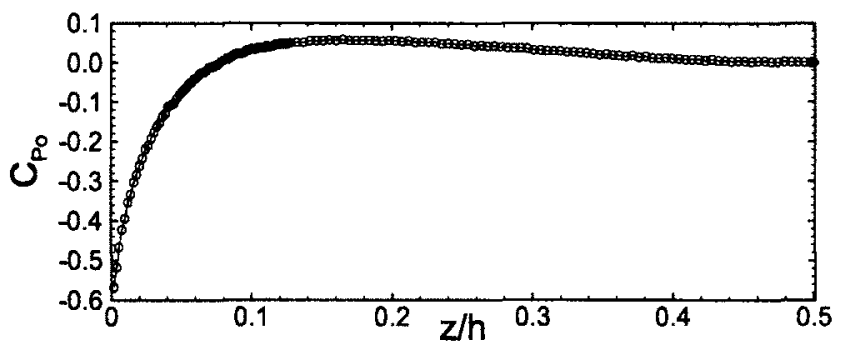

Figure 5.2: Spanwise variation of total pressure coefficient as measured with a Pitot probe 1.2 Bx upstream of the leading edge at $\mathrm{y} / \mathrm{s}=0.5$

summarized in Table 5.1 along with the outlet results.

\subsubsection{Blade Loading Distribution}

The overall blade loading (Zorić et al., 2007a, Zorić et al., 2007b, Hodson and Dominy, $1987 a$ ) and its distribution (Weiss and Fottner, 1995, Zorić et al., 2007a, Zorić et al., 2007b) are known to have significant influences on the size and strength of secondary flows. The midspan pressure distribution for PAKB is shown in Figure 5.3 as measured by Mahallati et al. (2007) using the same test section and turbulence generating grid as the current study. The loading distribution is plotted as static pressure coefficient $\left(C_{p}\right)$ versus fraction of surface length $\left(S / S_{\max }\right)$.

Figure 5.3 shows that varying the Reynolds number from $1.0 \times 10^{5}$ to $1.5 \times 10^{5}$ has only a small effect on the loading. The present study was conducted at a Reynolds number of $1.26 \times 10^{5}$ which falls nearly half way between the conditions shown. The aerofoil exhibits a "short" suction surface separation bubble (see Mayle, 1991) that extends from roughly $S / S_{\max }=0.61$ to $S / S_{\max }=0.78$, or $S / S_{\max }=0.74$ at Reynolds numbers of 100,000 and 150,000 , respectively. PAKB is considered to be an aft-loaded airfoil, with a suction peak at approximately $0.50 \mathrm{~S} / S_{\max }$. The suction surface boundary layer sees a gradual and continuous acceleration up to the suction peak. The pressure surface boundary layer sees a 
Table 5.1: Summary of PAKB results

\begin{tabular}{|c|c|c|c|}
\hline \multirow[b]{2}{*}{ LOCATION } & \multirow[b]{2}{*}{ FLOW QUANTITY } & \multicolumn{2}{|c|}{ ENDWALL } \\
\hline & & PLANAR & CONTOURED \\
\hline \multirow[t]{5}{*}{ INLET } & $\delta / h$ & \multicolumn{2}{|r|}{0.16} \\
\hline & $\delta^{*}[\mathrm{~mm}]$ & \multicolumn{2}{|r|}{1.9} \\
\hline & $\theta[\mathrm{mm}]$ & \multicolumn{2}{|r|}{1.6} \\
\hline & $H$ & \multicolumn{2}{|r|}{1.2} \\
\hline & $C_{P 0,1}^{\prime \prime}$ & \multicolumn{2}{|c|}{0.00025} \\
\hline OUTLET & $C_{P o_{\text {total }}}$ & -0.098 & -0.091 \\
\hline \multirow[t]{11}{*}{$B_{x}=1.40$} & $C_{P o_{\text {profile }}}$ & -0.057 & -0.054 \\
\hline & $C_{P o_{\text {secondary }}}$ & -0.041 & -0.037 \\
\hline & $Y_{\text {total }}$ & 0.043 & 0.039 \\
\hline & $Y_{\text {profile }}$ & 0.024 & 0.023 \\
\hline & $Y_{\text {secondary }}$ & 0.019 & 0.016 \\
\hline & $C_{S K E}^{\prime \prime}$ & 0.015 & 0.013 \\
\hline & $\alpha_{2}^{\prime \prime}[\mathrm{deg}]$ & -57.2 & -57.1 \\
\hline & $\alpha_{M S_{2}}^{\prime}[\mathrm{deg}]$ & -57.7 & -57.6 \\
\hline & $q_{2}^{\prime \prime} / q_{M S_{1}}^{\prime}$ & 2.304 & 2.326 \\
\hline & $q_{M S_{2}}^{\prime} / q_{M S_{1}}^{\prime}$ & 2.312 & 2.332 \\
\hline & $A V R$ & 0.979 & 0.988 \\
\hline \multirow[t]{10}{*}{ MIXED OUT } & $\overline{C_{P o_{\text {total }}}}$ & -0.117 & -0.108 \\
\hline & $C_{P o_{\text {profile }}}$ & -0.058 & -0.055 \\
\hline & $C_{P o_{\text {secondary }}}$ & -0.059 & -0.053 \\
\hline & $Y_{\text {total }}$ & 0.052 & 0.047 \\
\hline & $Y_{\text {profile }}$ & 0.025 & 0.024 \\
\hline & $Y_{\text {secondary }}$ & 0.027 & 0.023 \\
\hline & $\alpha_{2}^{\prime \prime}[\mathrm{deg}]$ & -57.7 & -57.6 \\
\hline & $\alpha_{M S_{2}}^{\prime}[\mathrm{deg}]$ & -57.7 & -57.6 \\
\hline & $q_{2}^{\prime \prime} / q_{M S_{1}}^{\prime}$ & 2.259 & 2.281 \\
\hline & $q_{M S_{2}}^{\prime} / q_{M S_{1}}^{\prime}$ & 2.309 & 2.329 \\
\hline
\end{tabular}

mild deceleration up to $0.3 S / S_{\max }$ where it begins to accelerate towards the trailing edge.

The static pressure difference across the blade passage drives the pressure leg of the horseshoe vortex towards the suction surface of the adjacent blade (Sieverding, 1985, Langston, 2001, Gregory-Smith, 1997). The application of endwall contouring is known to modify the static pressure distribution in the endwall region (Rose, 1994, Harvey et al., 2000) and affect the formation of the secondary flow (Hartland et al., 2000). The following discussion focuses on the changes to the flow field as interpreted from the limiting 


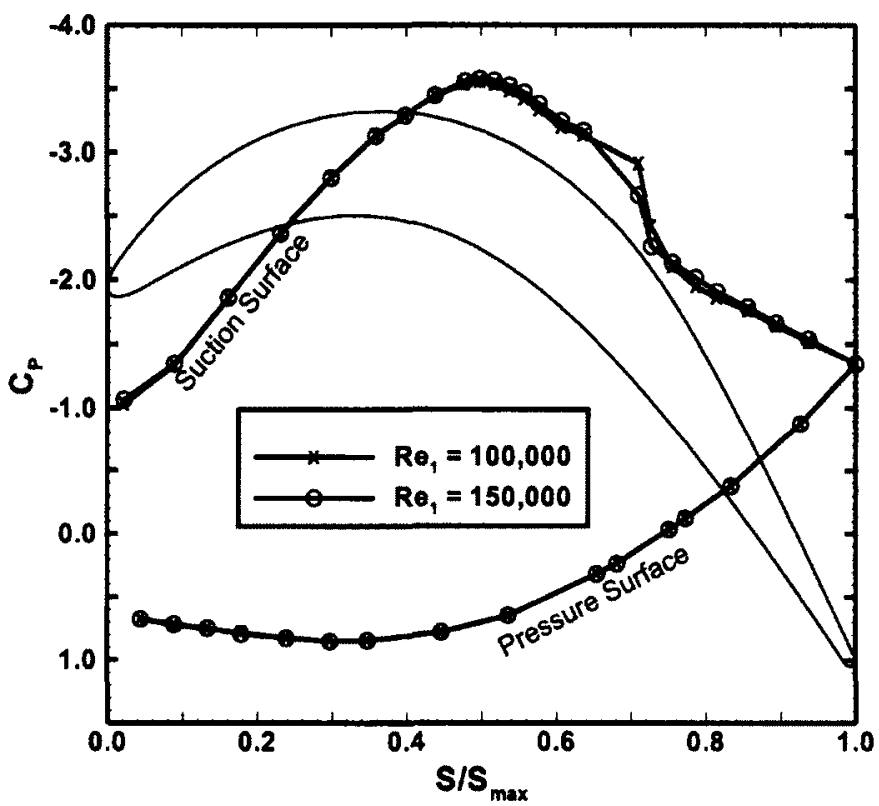

Figure 5.3: Loading distribution measured by Mahallati et al. (2007)

streamlines given by surface flow visualization.

\subsubsection{Flow Visualization Results}

Figure 5.4 shows oil surface flow visualizations overlaid with interpreted separation streamlines for the planar and contoured endwall configurations. The nomenclature of Sieverding (1985) is used wherever possible. On the planar endwall (Figure 5.4a), the flow enters the figure from the right and exits approximately through the top. The incoming stagnation streamline meets the primary separation line $S_{1}$ at the separation saddle point $A_{1} . S_{1}$ marks the separation of the inlet boundary layer from the endwall. The incoming inlet boundary layer fluid is funneled to either side of the blade where $S_{1}$ meets the suction surface. The second major separation line on the endwall, $S_{2}$, corresponds to the liftoff line of the horseshoe vortex. The horseshoe vortex is wrapped around the leading edge of the 
aerofoil downstream of $S_{2}$. The area between the airfoil leading edge and $S_{2}$ has the least pigment in every endwall photograph taken during the flow visualization study indicating that this is a region of very high shear stress. The formation of the oil flow pattern was observed through the Plexiglas wall after the wind tunnel operating point was set. It was seen that the first shearing of oil occurred away from the leading edge towards $S_{2}$ again suggesting that the surface shear stress is highest there. This is in agreement with the findings of Praisner and Smith (2006), who took measurements in the leading edge region of a symmetric bluff body. They found that the wall shear stress was highest in the reverse flow region beneath the horseshoe vortex.

The pressure legs of the two separation lines $\left(S_{1 \mathrm{P}}\right.$ and $\left.S_{2 \mathrm{P}}\right)$ travel across the blade passage; in doing so, the pressure leg of the horseshoe vortex corresponding to $S_{2 \mathrm{P}}$ evolves into the passage vortex while entraining endwall boundary fluid. $S_{1 \mathrm{P}}$ and $S_{2 \mathrm{P}}$ encounter the suction surface of the adjacent blade at high angle of attack; this results in what Sieverding (1985) refers to as a "strong interaction". The consequence of a strong interaction is the formation of the corner vortex where the suction-surface meets the endwall. The corner vortex is marked by $S_{3} . S_{3}$ appears to have its origin just downstream of where the suction leg of the primary inlet boundary layer separation $\left(S_{1 S}\right)$ meets the suction surface. Hodson and Dominy $(1987 b)$ found that the growth of the corner vortex can be seen as the distance between $S_{3}$ and the suction surface increases. The authors found that size and strength of the corner vortex are related to the strength of the cross-flow within the blade passage. The intensity with which the corner vortex is energized can be seen as surface streamlines are directed towards $S_{3}$ and the angle of incidence they make with it. The corner vortex would be energized most if the surface streamlines met the suction surface at a right angle. The new boundary layer that forms downstream of the pressure leg of the horseshoe vortex is swept towards the suction surface by the pitchwise cross-passage flow (shown schematically by the large arrow). Near the exit of the cascade at about mid-pitch, significant surface 
a)

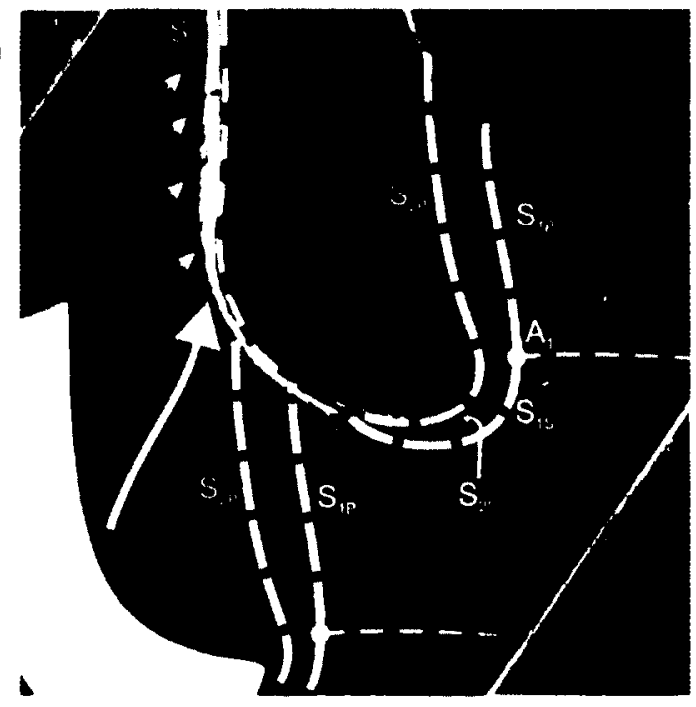

Planar

b)

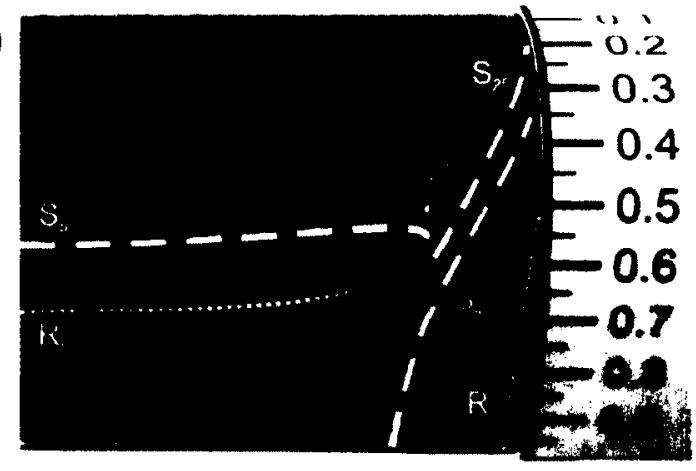

c)

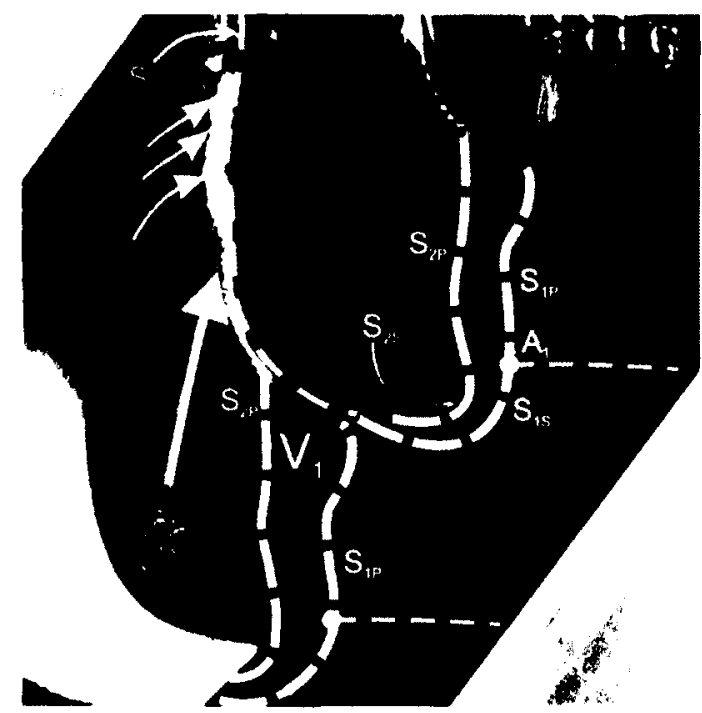

Contoured

d)

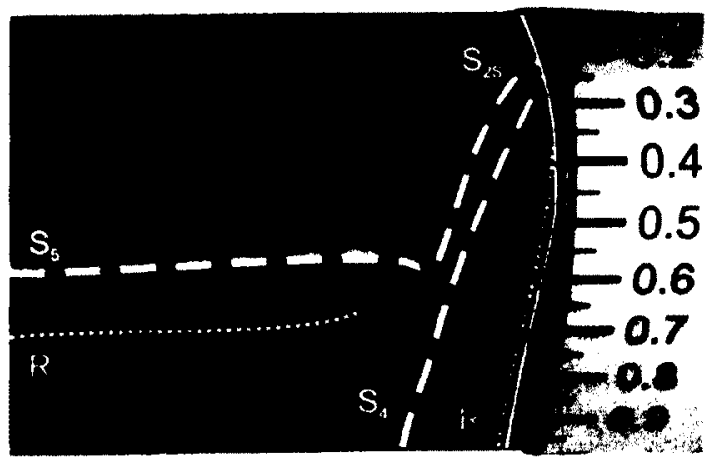

Figure 5.4: Endwall (a \& c) and suction surface (b \& d) oil surface flow visualization for the planar (a \& b) and contoured (c \& d) endwall configurations

streamline curvature occurs as the new boundary layer falls under the influence of the lower static pressure near the suction-surface/endwall-junction (shown by the small arrows).

The relationship between the passage vortex and the separation streamlines on the endwall can be seen in the CFD visualization of Figure 5.5. The flow enters the figure horizontally from left to right and is turned by the blades leaving through the bottom in the direction of the nominal exit flow angle. A plane aligned with the inlet design incidence contains a contour flood plot of total pressure deficit coefficient $\left(C_{P o}\right)$. The fiy-out magnifies 
the saddle point region. A distinct high loss region associated with the core of the suctionside leg of the horseshoe vortex is evident in the $C_{P_{o}}$ contour. The cores of the pressure-side and suction-side legs of the horseshoe vortex have been marked with streamlines coloured by local velocity. A singularity exists where the two legs meet and the flow is near stagnation there. The streamlines enter from the inlet and wrap themselves around the singularity numerous times before making their way into the core. The fly-out clearly shows how the secondary separation line $S_{2 \mathrm{P}}$ tracks the convection of the pressure-side leg of the horseshoe vortex across the passage as it evolves into the core of the passage vortex. The suction side leg follows the suction surface of the airfoil and convects toward midspan until it encounters the pressure leg of the horseshoe vortex from the adjacent airfoil where they intertwine. A red stream-ribbon shows how fluid from higher in the inlet boundary layer is also incorporated into the passage vortex.

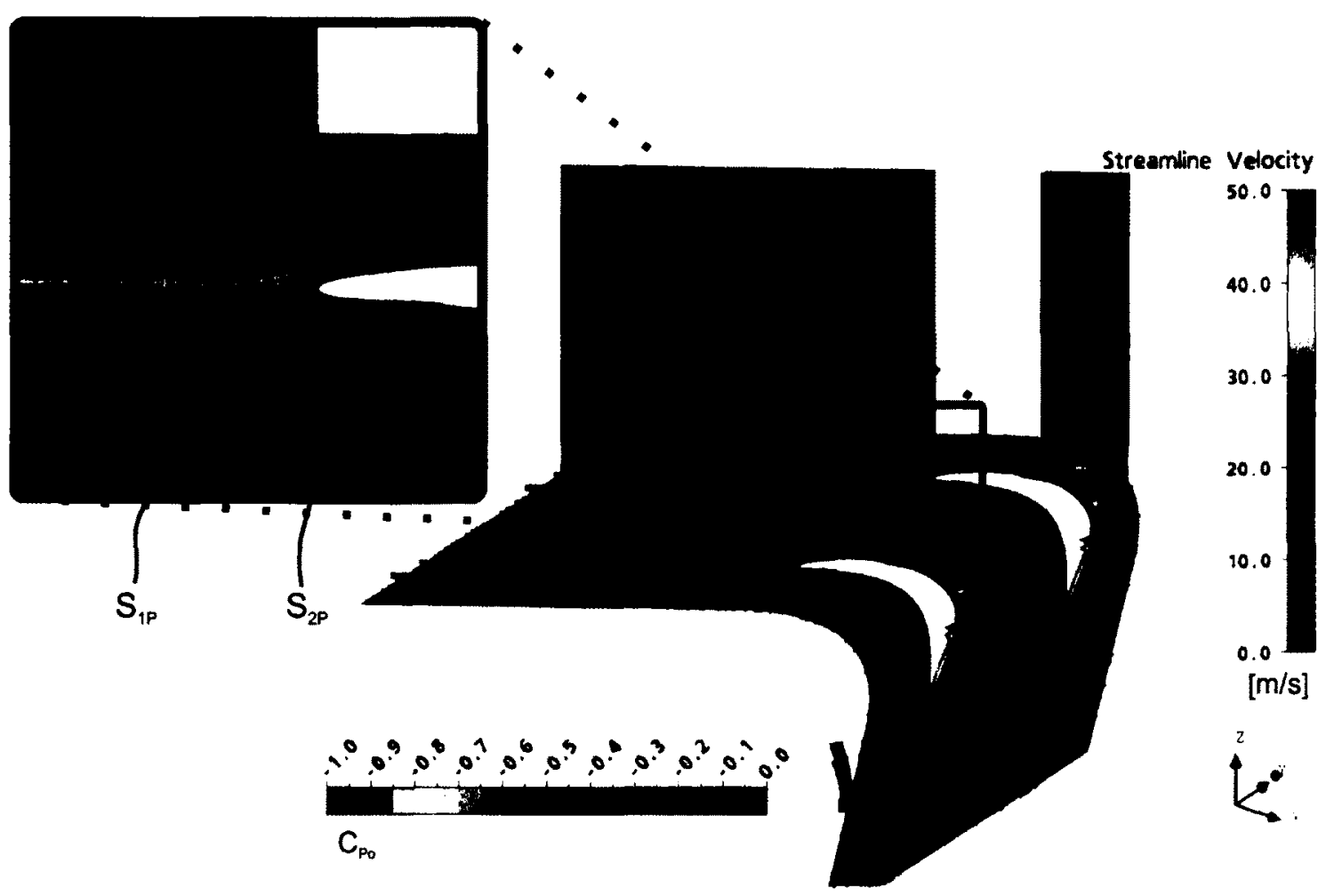

Figure 5.5: Visualization of passage vortex from CFD 
The suction surface flow visualization is shown Figure $5.4 \mathrm{~b}$ with a scale marking the approximate fraction of suction surface length from the leading edge. Figure $5.4 \mathrm{~b}$ shows three dominant separation lines and two reattachment lines. $S_{5}$ and $R_{5}$ mark the separation and reattachment of the suction surface boundary layer. The reattachment of the corner vortex separation $\left(S_{3}\right)$ is marked by $R_{3} . S_{4}$ is typically associated with the passage vortex, while $S_{2 S}$ is the extension of the suction leg of the horseshoe vortex that has been swept off the endwall. As the suction leg of the horseshoe vortex works its way towards the trailing edge, it convects towards midspan until it encounters the suction surface separation $\left(S_{5}\right)$ where it appears to vanish. The smoke flow visualizations of Wang et al. (1997) have shown that the suction leg can be lifted from the endwall by the passage vortex and wrapped around it. This behaviour is supported by the CFD visualization discussed above and has also been suggested by other researchers (Sharma and Butler, 1987, Benner et al., 2004b, Sieverding and Van Den Bosche, 1983).

The suction surface flow visualizations show an appreciable distance between the $S_{2 \mathrm{~S}}$ and $S_{4}$ separation lines. Several researchers (Hodson and Dominy, 1987b, Denton, 1993, Wang et al., 1997, Benner et al., 2004b) suggest that this region is filled with inlet boundary layer fluid that has been directed there by the primary inlet boundary layer separation $S_{1}$. In the present study, when the endwall flow visualizations were conducted and no oil was applied to the suction surface of the blade, most of the oil from the endwall pooled against $S_{3}$ and was slowly convected towards the trailing edge. This buildup of oil is clearly seen in Figure 5.4a. On occasion it was observed that a small amount of oil was swept off the endwall onto the suction surface of the airfoil and was convected towards the trailing edge between $S_{2 S}$ and $S_{4}$. It is therefore believed that the wall induced vortex as shown in Figure 5.6 is comprised of inlet boundary layer fluid.

The application of the contoured endwall did not change the broad flow physics given that the separation and reattachment lines of Figure $5.4 \mathrm{a}$ and $5.4 \mathrm{~b}$ are also seen in the 
oil surface flow visualizations of Figure $5.4 \mathrm{c}$ and $5.4 \mathrm{~d}$. Consequently the same vortical structures are present in both flows. The contouring affects the local static pressure on the endwall by means of streamline curvature (Rose, 1994). A convex curvature locally accelerates the flow thus reducing the local static pressure. A concave wall curvature causes a relative diffusion of the flow thereby raising the local static pressure. Valley $V_{1}$ (Figures 5.1 and 5.4c) represents one such region of concave curvature, and the local diffusion of the flow can be seen by the increased distance between $S_{1 \mathrm{P}}$ and $S_{2 \mathrm{P}}$ relative to Figure 5.4a. The increased static pressure, compared to that for the nominal endwall height, caused the initial separation of the inlet boundary layer (marked by $S_{1 \mathrm{P}}$ ) to move upstream. When observing the formation of the pattern in that region, it was found that the oil approached stagnation at the upstream turning of $S_{1 \mathrm{P}}$ and was slowly swept towards the suction surface along the separation line. This feature is similar in appearance to the endwall boundary layer separation of Ingram et al. (2005) but does not seem to harm performance.

The contouring reduces the cross-passage flow and influences the formation of the corner vortex marked by $S_{3}$. As with the planar case, $S_{3}$ has its origin downstream of where $S_{1 S}$ meets the suction surface; however, the corner vortex remains small over a much larger fraction of the suction surface. The new intra-passage boundary layer that forms downstream of $S_{2 \mathrm{P}}$ is relatively un-swept compared to the planar case (shown by the large arrow). The endwall flow visualizations of Ingram et al. (2002) also showed a reduction in cross flow for both contoured endwalls tested. In Figure $5.4 \mathrm{c}$ of the current work, the angle of incidence of the intra-passage boundary layer is almost tangent to the suction surface. This results in a "weak convergence" (Sieverding, 1985) that imparts little energy to the corner vortex. Just downstream of the large arrowhead, the contouring begins to blend back to the nominal endwall height in the direction of the outlet metal angle. In doing so the flow is accelerated, the static pressure drops, and the surface streamlines turn abruptly toward the suction surface of the blade. As a result the corner vortex is energized. A similar 
increase in cross-flow near the exit of the cascade was documented by the endwall surface flow visualizations of Hartland et al. (2000) for their contoured endwall. The influence of the contouring on the cross-passage flow and its effect on the roll-up of the passage vortex will be discussed further with regards to the pneumatic probe measurements presented below.

The suction surface oil flow visualization for the contoured endwall is presented in Figure 5.4d. The endwall intersection is shown by the solid white line since the blades are recessed into the contoured endwall. In comparison to the planar case, $S_{2 S}$ and $S_{4}$ are driven further towards midspan near their origins by the higher local static pressure at the suctionsurface/endwall-junction resulting from the presence of valley $V_{1}$. The penetration height of $S_{4}$ at the trailing edge has been reduced; this is a sign of a reduction in passage vortex size.

\subsubsection{Pressure Probe Results}

Figure 5.6a shows line contours of total pressure loss coefficient overlaid with a colour flood of streamwise vorticity coefficient as measured at the trailing edge plane for the planar endwall. The view is looking upstream with the passage suction surface on the left and pressure surface on the right. The streamwise vorticity is derived from the incompressible Euler equations as per Gregory-Smith et al. (1988). It is a property that is useful for identifying vortical structures and quantifying their strength and sense of rotation. For the right-handed coordinate system selected, the passage vortex has negative vorticity (clockwise rotation) about the streamwise direction. Two counter-rotating vortical structures are evident: the corner vortex located where the suction surface meets the endwall, and the wall induced vortex located near the blade suction surface beginning at about $8 \%$ of span.

Figure $5.6 \mathrm{~b}$ shows the interpretation of the vortical structures as they exist just upstream 
(a)
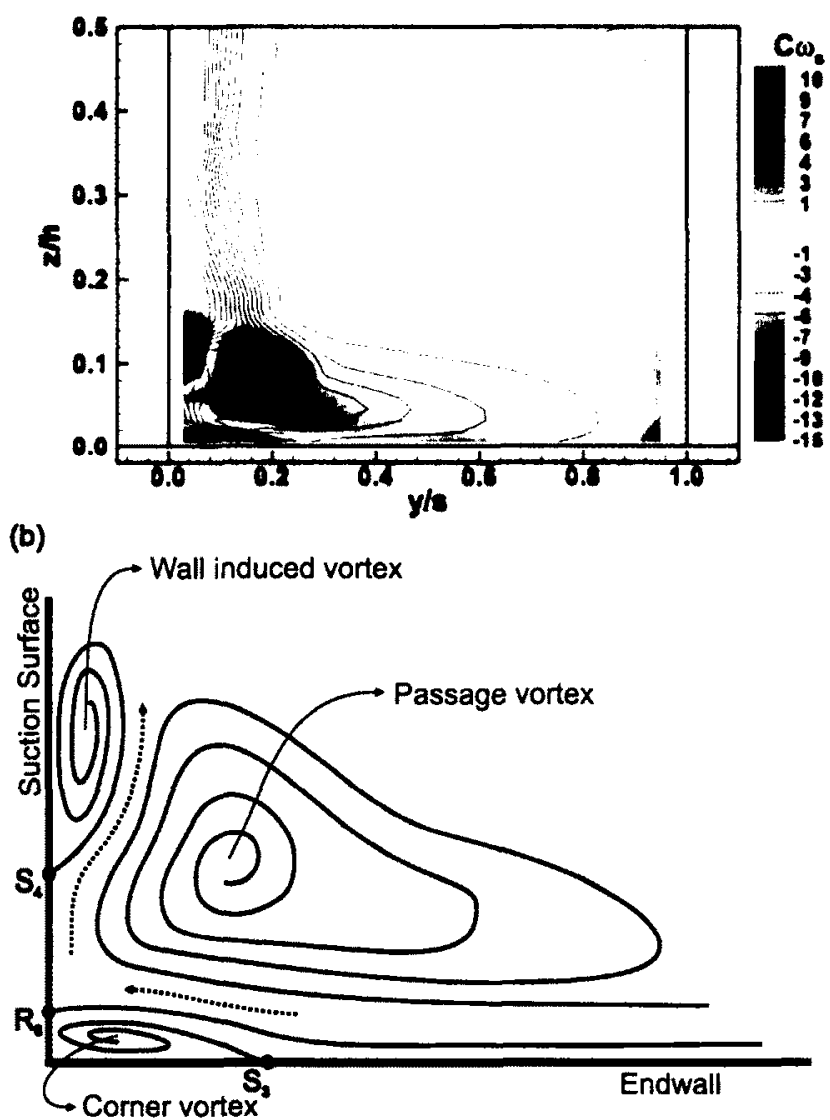

Figure 5.6: (a) Line contours of total pressure loss coefficient superimposed with flood of streamwise vorticity and (b) interpreted vortex structure at the trailing edge plane (1.00Bx)

of the trailing edge plane of the cascade and their relationship to the separation and reattachment lines discussed above. The corner vortex is bound by $S_{3}$ and $R_{3}$ and has a sense of rotation opposite to that of the passage vortex. The wall induced vortex separates at $S_{4}$ and also has a counterclockwise sense of rotation. The dotted arrows show the direction in which it is believed that low momentum fluid is convected into the secondary flow structure. The application of endwall contouring is thought to reduce this convection process leading to lower losses. This will be discussed with relation to the pressure probe measurements presented next. 
The seven-hole probe traverse data for the $0.63 B_{x}$ plane are presented in Figure 5.7. The solid line thumbnail in the centre of the figure indicates the location and pitchwise extent of the measurement plane. The figure contains two different types of plots showing the modifications to the flow field caused by the application of endwall contouring. Secondary velocity vectors are superimposed with a flood of negative streamwise vorticity coefficient $\left(C_{\omega s}\right)$ for the baseline and contoured endwall in Figures $5.7 \mathrm{a}$ and $5.7 \mathrm{c}$, respectively. The secondary velocity vectors show the components of velocity on a plane normal to the massaveraged flow velocity vector (primary flow direction). Vorticity is convected with fluid particles, thus enabling these plots to track the passage vortex in subsequent figures. Note that the peak of negative vorticity (Fig. 5.7a) does not coincide with the point about which the secondary velocity vectors revolve. The profiled endwall results in Figure 5.7c exhibit a weaker passage vortex; no distinct peak of negative vorticity is obvious.
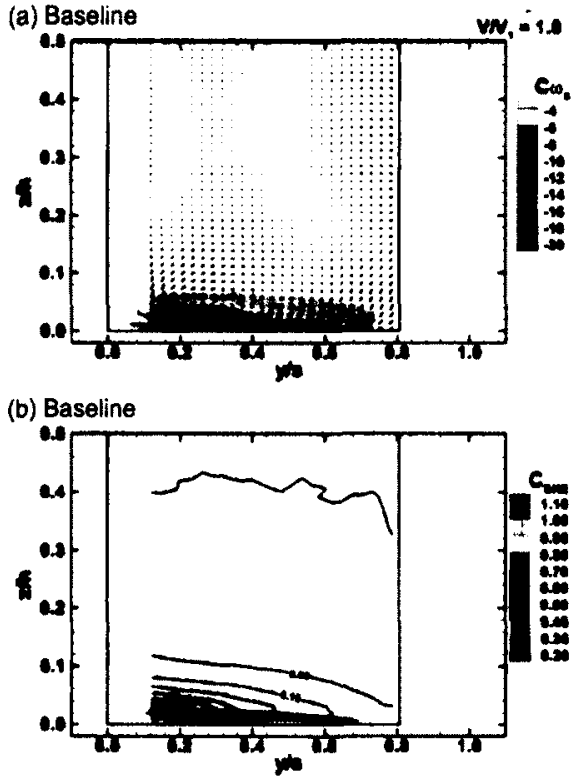
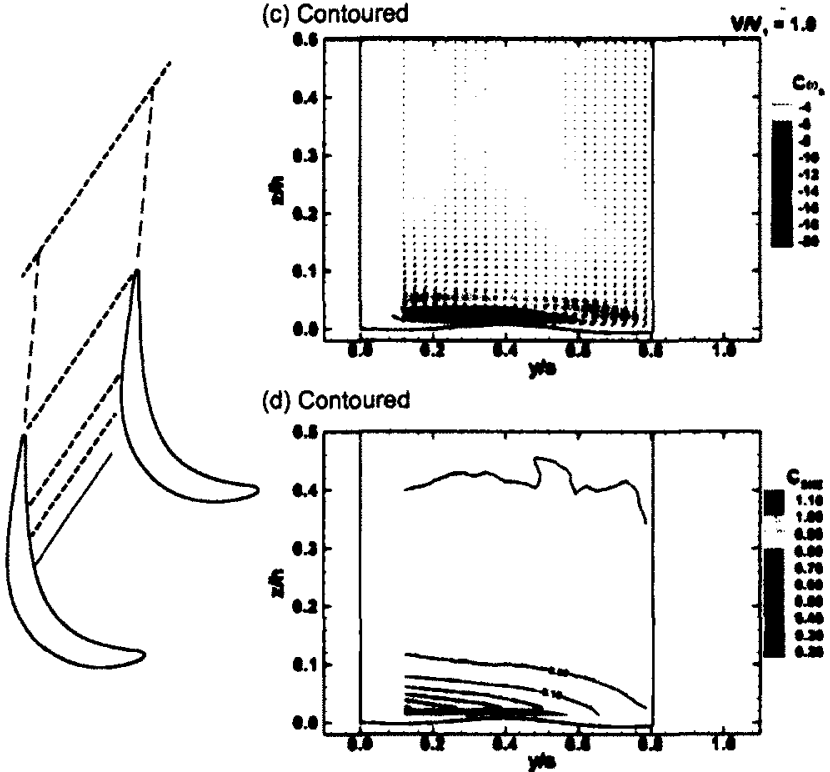

Figure 5.7: Secondary velocity vectors with flood of streamwise vorticity coefficient $\left(C_{\omega s}\right)(\mathbf{a} \& \mathbf{c})$, and line contours of total pressure loss coefficient $\left(C_{P o}\right)$ with flood of secondary kinetic energy coefficient $\left(C_{S K E}\right)(\mathrm{b} \& \mathrm{~d})$, at $63 \%$ axial cord

The other plots in Figure 5.7 (5.7b and 5.7d) display line contours of total pressure 
loss coefficient $\left(C_{P o}\right)$ overlaid with a flood of secondary kinetic energy coefficient $\left(C_{S K E}\right)$. The secondary kinetic energy coefficient is the kinetic energy associated with the secondary velocity vectors normalized by the kinetic energy on the inlet centerline of the cascade. Thus $C_{S K E}$ illustrates the strength of the cross-flow near the endwall, whose peak is approximately 2.7 times stronger for the planar endwall. The pitchwise cross-flow is believed to be responsible for rolling up the boundary layer near the endwall, and this process appears more advanced for the planar case judging from the $C_{P o}$ contours of Figures $5.7 \mathrm{~b}$ and $5.7 \mathrm{~d}$. Hartland et al. (2000) also found weaker cross-flows and less convection into the suction surface corner resulting from the application of their profiled endwall; they attribute this to an alleviation of the cross-passage static pressure gradient in the early part of the passage.
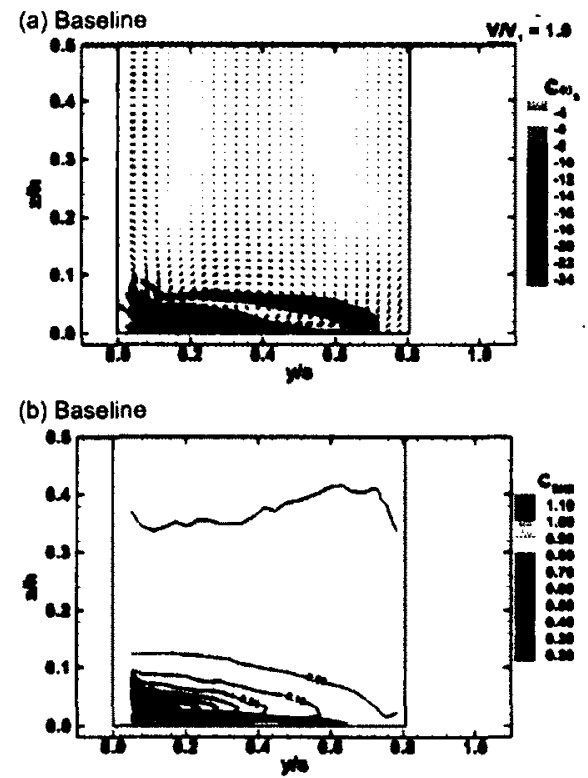
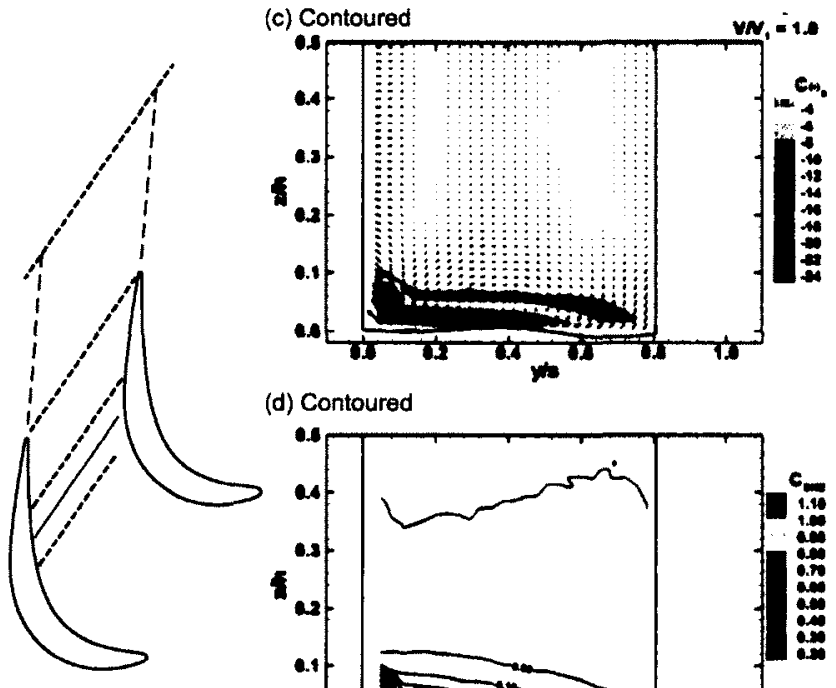

(d) Contoured

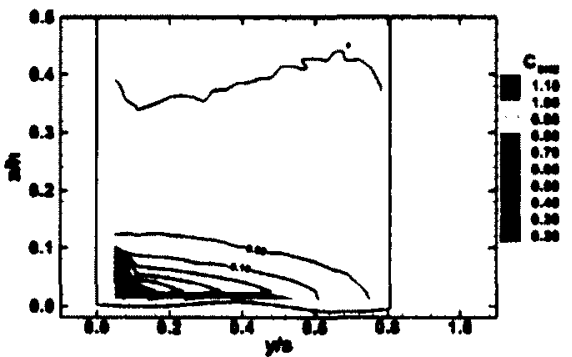

Figure 5.8: Secondary velocity vectors with flood of streamwise vorticity coefficient $\left(C_{\omega s}\right)(\mathbf{a} \& \mathbf{c})$, and line contours of total pressure loss coefficient $\left(C_{P o}\right)$ with flood of secondary kinetic energy coefficient $\left(C_{S K E}\right)$ (b \& d), at $71 \%$ axial cord

Figure 5.8 shows the secondary flow structures at $71 \%$ axial chord. The passage vortex has strengthened for the planar endwall in Figure 5.8a relative to Figure 5.7a (note the change in scale for the $C_{w s}$ flood contours). The contoured case (Figure 5.8c) is beginning 
to show a peak of negative vorticity near the suction surface at about $z / h=0.06$, yet its strength is weaker than that for the planar endwall. In Figure 5.8b, the planar endwall exhibits stronger pitchwise cross-flow as measured by $C_{S K E}$, and the roll-up of the secondary flow is more advanced because of the high secondary kinetic energy that existed upstream near the endwall in Figure 5.7b.

In Figure $5.8 \mathrm{~b}$, the core of high total pressure loss is starting to rise away from the endwall, displaced by higher energy fluid convected from the pressure side of the passage. The bottom left corner of Figure $5.8 \mathrm{~b}$ shows a high loss region, marking the existence of the corner vortex. The presence of the corner vortex is expected at this axial location judging from the surface flow visualizations of Fig 5.4a. The loss contours of Figure 5.8d show no evidence of a corner vortex or an appreciable loss core associated with the roll-up of the boundary layer.

Figure 5.9 shows the traverse results from a plane located at $80 \%$ axial chord. In Figure 5.9a, the planar endwall has a large kidney shaped mass of fluid with high negative vorticity, while the contoured endwall generates two distinct accumulations of fluid with negative vorticity of lower magnitudes (Figure 5.9c). The contours of $C_{S K E}$ for the planar endwall show two distinct peaks. The first, located near the endwall at $y / s=0.22$, is linked to the pitchwise cross-flow as discussed above. The second peak, located near the suction surface at $z / h=0.06$, is related to spanwise flow away from $R_{3}$ towards midspan.

Examining the loss contours of Figure $5.9 \mathrm{c}$ reveals a lobe of lossy fluid marked by the $C_{P o}=-0.50$ line that is displaced away from the suction surface of the blade by fluid that has been convected into the corner, across the limiting streamline connecting $S_{3}$ and $R_{3}$, and up the blade suction surface. To the authors' knowledge, this has not been documented previously.

The secondary kinetic energy coefficient in Figure 5.9d has a peak value approximately 

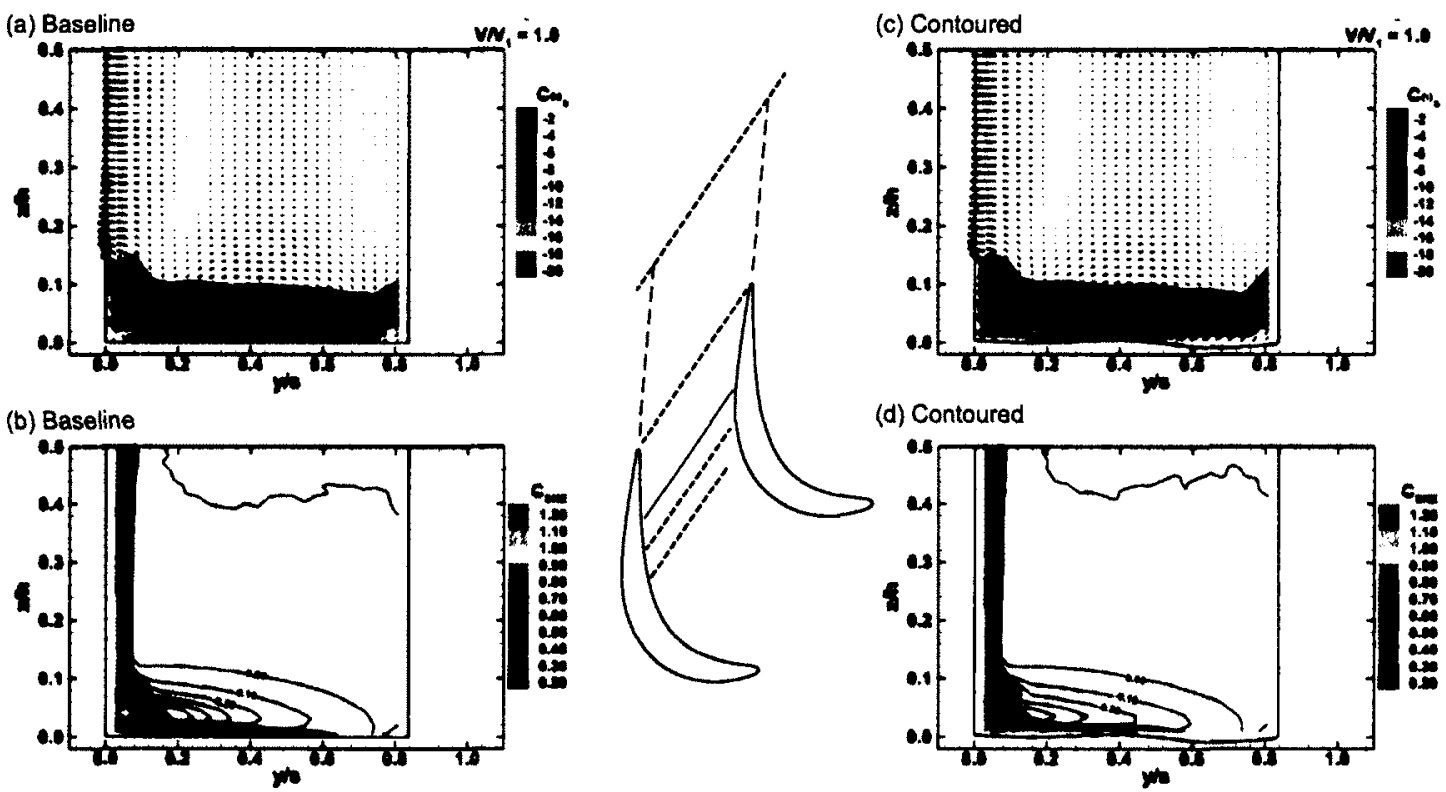

Figure 5.9: Secondary velocity vectors with flood of streamwise vorticity coefficient $\left(C_{\omega s}\right)(\mathbf{a} \& \mathbf{c})$, and line contours of total pressure loss coefficient $\left(C_{P_{o}}\right)$ with flood of secondary kinetic energy coefficient $\left(C_{S K E}\right)$ (b \& d), at $80 \%$ axial cord

$68 \%$ that for the planar endwall case. This peak is associated with spanwise flow up the blade suction surface. Also noteworthy is the appearance of the corner vortex forming at the suction-surface/endwall-junction, but its development has been delayed relative to the planar case. The delayed appearance of the corner vortex was discussed above in relation to the flow visualization results of Figures $5.4 \mathrm{a}$ and $5.4 \mathrm{c}$.

By the trailing edge plane the contoured endwall is blended back to the nominal endwall height. This has a significant effect on the pitchwise cross-flow, which is now stronger for the contoured endwall for the first time (Figure 5.10d). The strong cross-flow energizes the corner vortex, which in a short streamwise distance has grown to a size and strength comparable to that of the baseline case. Nevertheless, the planar endwall has stronger spanwise flow associated with the peak of $C_{S K E}$ located near the suction surface at $z / h=$ 0.08 (Figure 5.10c), that will continue to convect fluid away from the endwall downstream 
of the blade passage. The application of endwall contouring results in a $31 \%$ reduction in peak strength of the passage vortex at the exit plane of the cascade based on the streamwise vorticity.
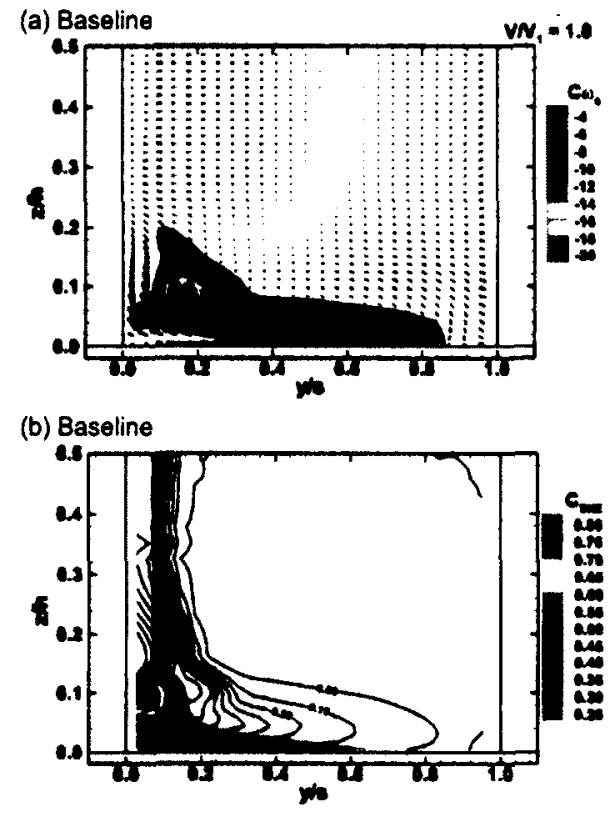

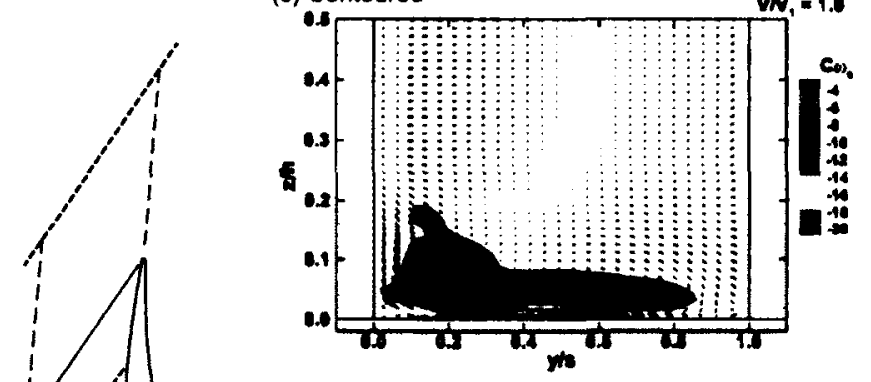

(d) Contoured

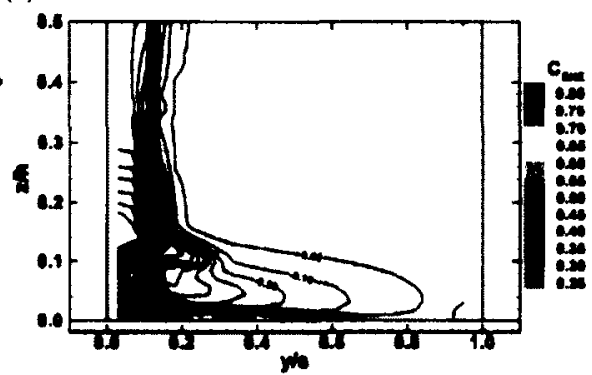

Figure 5.10: Secondary velocity vectors with flood of streamwise vorticity coefficient $\left(C_{\omega s}\right)$ (a \& c), and line contours of total pressure loss coefficient $\left(C_{P o}\right)$ with flood of secondary kinetic energy coefficient $\left(C_{S K E}\right)$ (b \& d), at the trailing edge plane

The exit flow was measured $140 \% B_{x}$ downstream of the leading edge of the cascade and the results are presented in Figure 5.11. The projection of the trailing edges are shown as dashed lines at $y / s=0$ and $y / s=1$. Overall the flow fields for the two test cases appear similar, but several features are worth discussing. The peak negative vorticity that is associated with the passage vortex has been reduced by about $10 \%$ by the profiled endwall. The area of the traverse plane that contains fluid of negative vorticity is also smaller, and thus it can be said that the contouring reduces the overall size and strength of the passage vortex. To the left, and slightly above the passage vortex is a core of fluid with positive vorticity. The origin of this fluid is unclear; however, it has been suggested that it is shed 
vorticity from the trailing edge (Hodson and Dominy, 1987b), or that it is the wall induced vortex described above (Benner et al., 2004b). The peak strength of this counterclockwise rotating vortex has been reduced by about $20 \%$ relative to the planar endwall case. The fact that the peak vorticity of these two structures is linked suggests a physical connection between them. It is plausible that this positive vorticity is generated in the blade suction surface boundary layer between $R_{3}$ and $S_{4}$ (see Figure 5.6b), induced by the passage vortex. Near the endwall and to the left of the trailing edge projection lines, there are two cores of positive vorticity. These are the corner vortices of the passage measured (near $y / s=0$ ) and the adjacent passage in the cascade (near $y / s=1$ ). The strength of the corner vortex is comparable in Figures 5.11a and 5.11c.
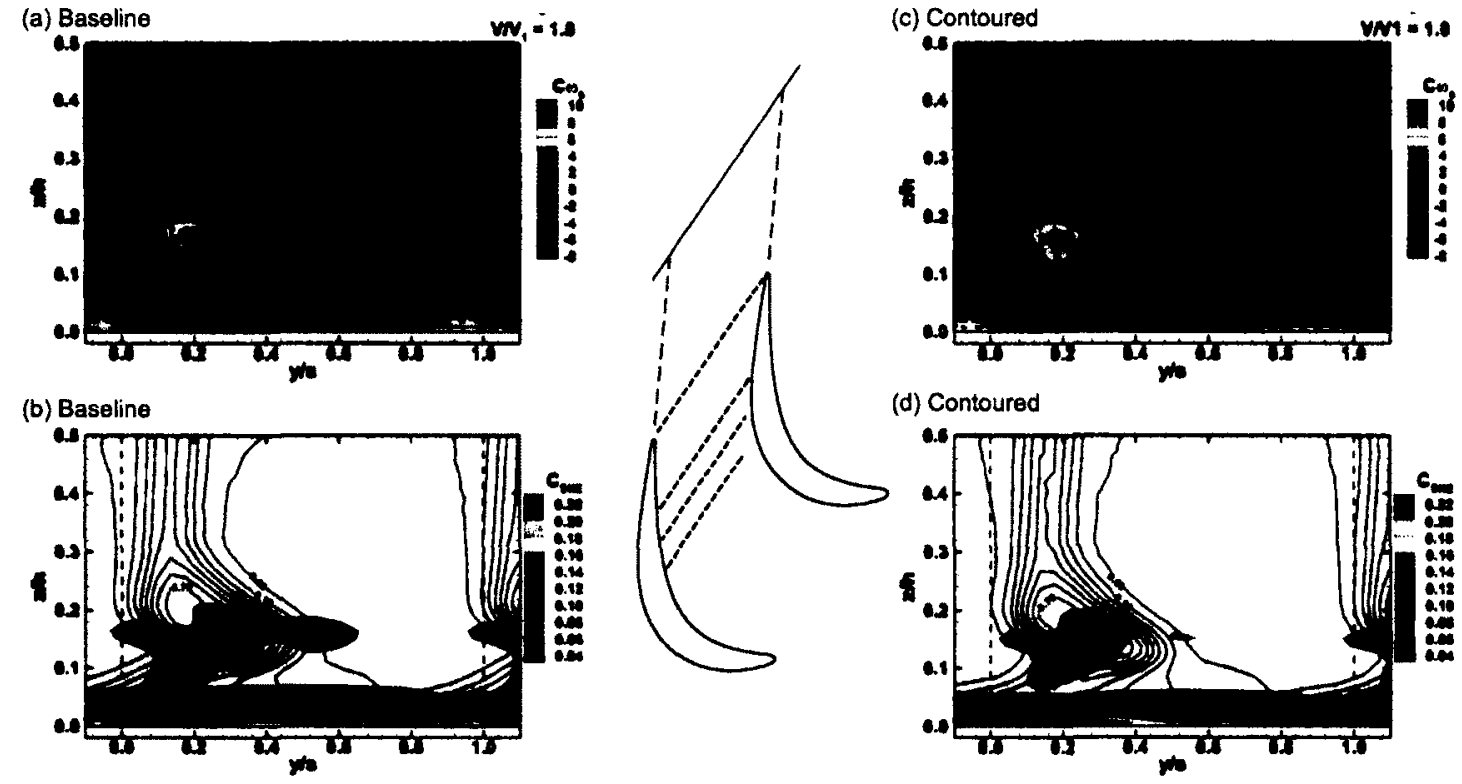

(d) Contoured

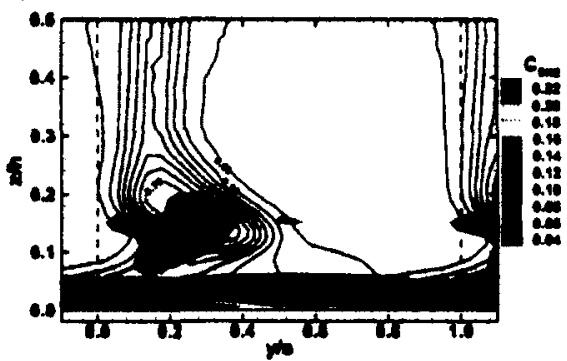

Figure 5.11: Secondary velocity vectors with flood of streamwise vorticity coefficient $\left(C_{\omega s}\right)$ (a \& $\left.\mathbf{c}\right)$, and line contours of total pressure loss coefficient $\left(C_{P_{0}}\right)$ with flood of secondary kinetic energy coefficient $\left(C_{S K E}\right)$ (b \& d), at $140 \%$ axial cord

The colour flood contour plots of secondary kinetic energy coefficient in Figure 5.11 display the same trend that had developed by the trailing edge plane. Near the endwall, 
the contouring generates higher secondary kinetic energy resulting from the overturning developed in the aft part of the passage. Away from the endwall, a distinct peak of secondary kinetic energy exists between the passage and counter vortices. This kinetic energy appears to belong to the spanwise flow up the blade suction surface that was evident at the trailing edge. Inspection of the secondary velocity vectors of Figure 5.11 reveals that the spanwise flow has been turned clockwise, and that the degree of turning seems proportional to the strength of the passage vortex.

The total pressure loss contours of Figures $5.11 \mathrm{c}$ and $5.11 \mathrm{~d}$ have peaks of similar magnitudes except along the endwall. The highest losses for both the flat and profiled cases occur near the endwall, marked by the liftoff of the corner vortex. The next loss core from the endwall, located further towards midspan is linked to the passage vortex. The peak loss in these cores is almost identical for the two cases (within the error of the experiment). The most substantial difference in loss is seen in the third loss core located farthest from the endwall and roughly in line with the wake. This high loss fluid is comprised of suction surface boundary layer fluid that has been displaced by the passage vortex, and fluid that has been convected into the suction corner and up the blade surface by the cross-flow and spanwise flow. It is unclear what happens to the wall induced vortex at the trailing edge of the blade; it may find itself in this loss structure, or it might be wrapped around the passage vortex. In the latter case, its location could be lost due to the resolution of the measurement grid. The peak loss of this third structure is comparable in the planar and contoured configurations; however the size of the structure has been reduced with the contouring. The reduction in size is believed to be a result of the presence of less convected fluid, as suggested by the dashed arrows of Figure $5.6 \mathrm{~b}$.

The exit flow of the cascade is analyzed by pitchwise mass-averaging in Figure 5.12a. The total pressure loss coefficient is normalized by the outlet dynamic pressure at midspan. The contouring produces slightly higher losses from the wall out to $10 \%$ span, and the two 
configurations have almost equal loss over the next $6 \%$. From $16 \%$ to $34 \%$ of span, an appreciable loss reduction is achieved by the profiled endwall, as discussed above. Between $32 \%$ and midspan, a slight increase in loss is measured for both configurations as a result of the inlet total pressure non-uniformity discussed in regards to Figure 5.2. The massaveraged and mixed-out mass-averaged exit flow results are summarized in Table 5.1. The endwall contouring reduces the mixed-out total pressure loss coefficient $Y_{\text {total }}$ by $9.3 \%$ and mixed out $Y_{\text {secondary }}$ was reduced by $15.8 \%$.

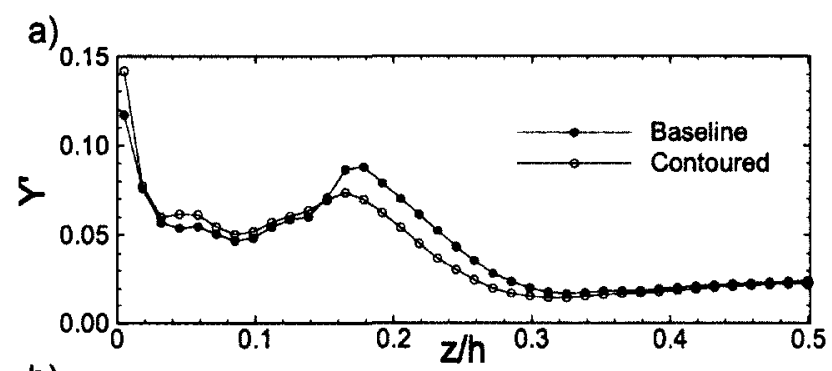

b)
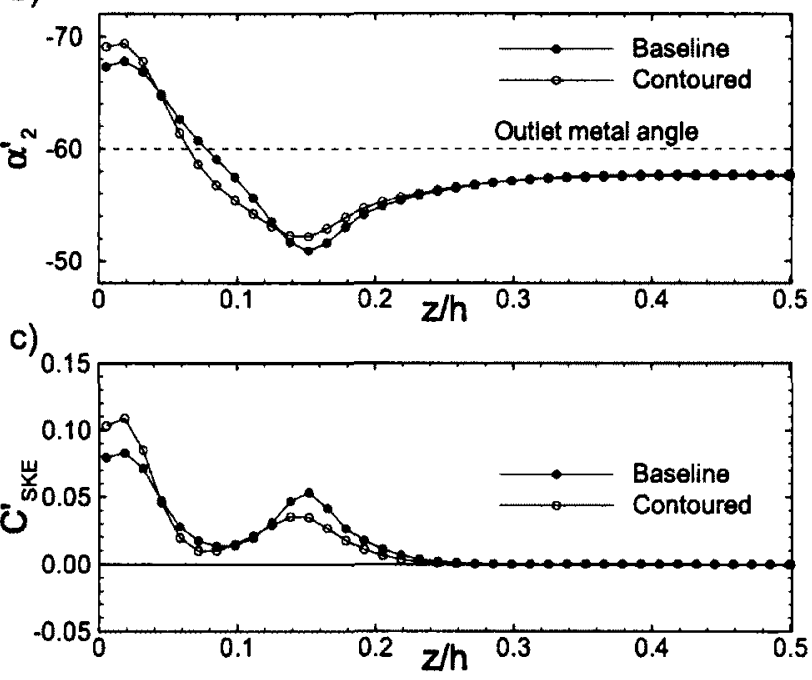

Figure 5.12: Pitchwise mass-averaged results for the $140 \% B_{x}$ measurement plane; Total pressure loss coefficient (a), outlet flow angle (b) and secondary kinetic energy coefficient (c)

The outlet flow-angle distribution in Figure $5.12 \mathrm{~b}$ shows that the blade row produces a classic over/underturning of the flow. The application of contouring increases the 
overturning at the endwall by 1.8 degrees and reduces the maximum underturning by 1.3 degrees. The mixed-out mass-averaged flow angle was unchanged within the uncertainty of the experiment.

The spanwise secondary kinetic energy distribution for the blade row is presented in Figure 5.12c. The high $C_{S K E}$ near the endwall is a direct consequence of the flow overturning there. The peak between $z / h=0.1$ to $z / h=0.2$ results from the underturning as well as the spanwise flow as discussed above. At the downstream measurement plane, endwall profiling has the effect of increasing the secondary kinetic energy near the endwall and reducing it between $10 \%$ and $20 \%$ of span. Overall, the measured mass-averaged $C_{S K E}$ has been reduced by $13.3 \%$.

\subsection{Computational Results}

\subsubsection{Blade Loading Distributions}

Figure 5.13 shows the predicted loading distributions for the planar and contoured endwall configurations at four spanwise locations. There is little influence of the contouring above $10 \%$ span (Figures 5.13a and 5.13b). At 10\% span (Figure 5.13c) the static pressure on the aft portion of the suction surface is increased by the presence of the contouring, resulting in a reduction of the blade loading coefficient $\left(C_{P-L}\right)$. This change occurs between the $R_{3}$ reattachment line and the $S_{4}$ separation, where the suction surface boundary layer is strongly influenced by the passage vortex. The details of this interaction will be discussed in the following subsection in regard to Figure 5.15.

At $5 \%$ span (Figure 5.13d) the peak static pressure is increased, again resulting in a reduction of $C_{P-L}$. This is a result of the relative diffusion caused by the depression in the endwall $\left(V_{1}\right)$ at the junction with the suction surface. As the depression blends out 


a)

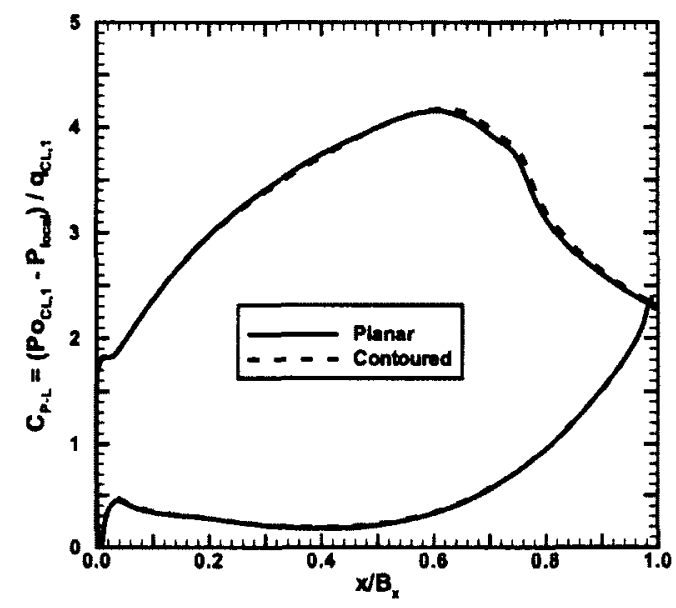

b)

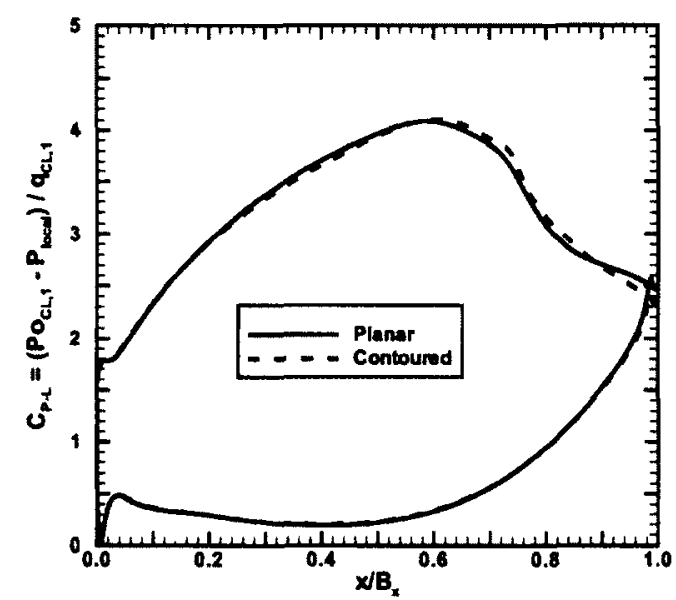

c)

$10 \%$ Span

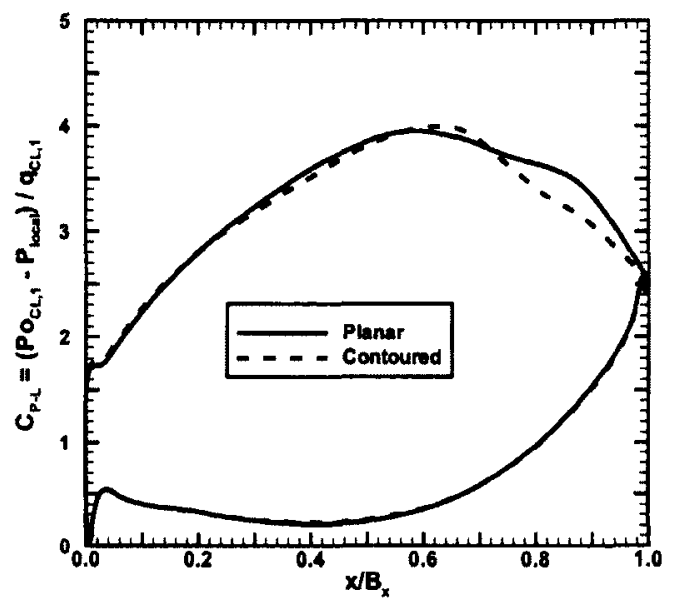

d)

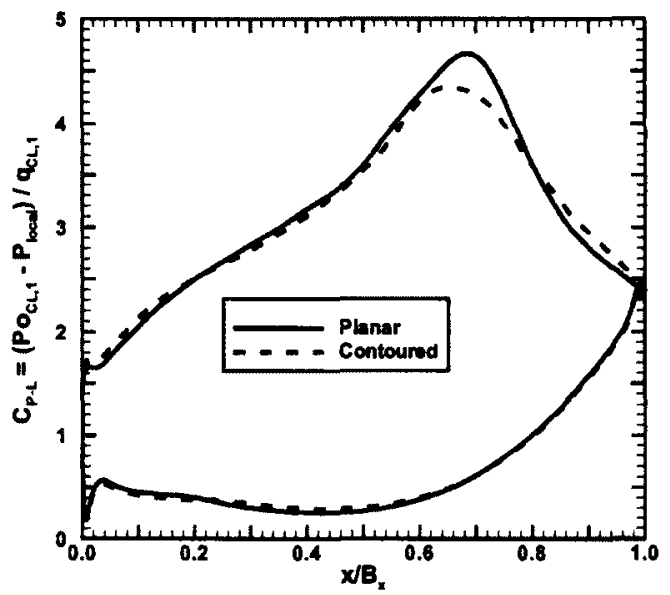

Figure 5.13: Predicted distributions of blade surface static pressure at various spanwise locations

in the direction of the outlet metal angle near $0.80 B_{x}$, a slight decrease in static pressure as seen relative to the planar endwall case. The variations in loading seen in Figure 5.13 are consistent with data presented by Hartland et al. (2000) for their linear cascade, and Brennan et al. (2003) for the redesign of the Rolls-Royce Trent test rig. 


\subsubsection{Flow Visualization}

The predicted endwall surface shear-stress trajectories are compared to the experimental oil flow visualizations for the planar and contoured configurations in Figure 5.14. In general the changes in the flow field are captured by the computations. The predicted effect of the contouring on the pressure side leg of the inlet boundary layer separation, marked by $S_{1 \mathrm{P}}$ is in agreement with that seen experimentally. $S_{1 \mathrm{P}}$ is moved upstream by the higher static pressure that results from the presence of valley $V_{1}$. The computations appear to over predict the effect of the contouring on the location of $S_{2 \mathrm{P}}$ that marks the location of the pressure side leg of the horseshoe vortex as it is swept across the passage. In Figure $5.14 \mathrm{~d}$, the prediction shows that the passage vortex is directed further pitchwise than is inferred from the oil surface flow visualization (5.14c). However, this could be a result of a misinterpretation of the oil flow. Downstream of $S_{1 \mathrm{P}}$ and between the saddle point and suction surface of the adjacent blade, the surface shear stress is low. The location of $S_{2 P}$ was estimated by examining a series of test cases having oil mixtures of different viscosities. This could have lead to some inaccuracy in estimating its exact location.

At the junction of the pressure surface with the endwall, in the location of the pressure surface separation bubble, the computations predict a region of stagnated recirculating fluid (Figure 5.14b). This recirculation is reduced by the protrusion of the contouring at that location. The recirculation and its reduction are not evident in the experimental oil flow visualizations of Figures 5.14a and 5.14c, but this could be a result of the low surface shear stress there.

The effect of the contouring on the new boundary layer forming on the endwall downstream of $S_{2 \mathrm{P}}$ is captured by the simulations. For the planar endwall configuration (Figure 5.14b) the new boundary layer is swept in the direction of the pressure surface of the adjacent blade by the pitchwise cross flow as shown by the large arrow. This happens to 
a)
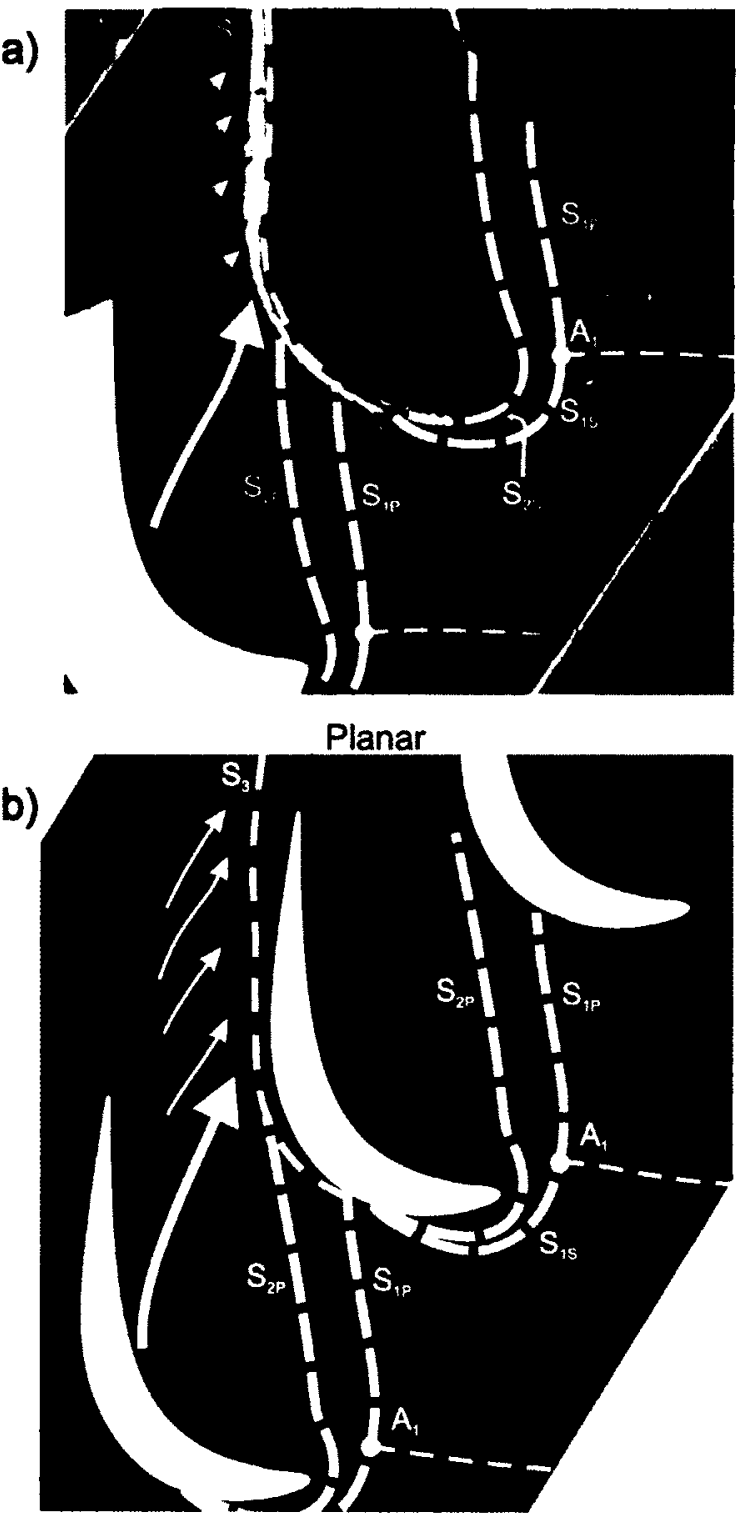

c)

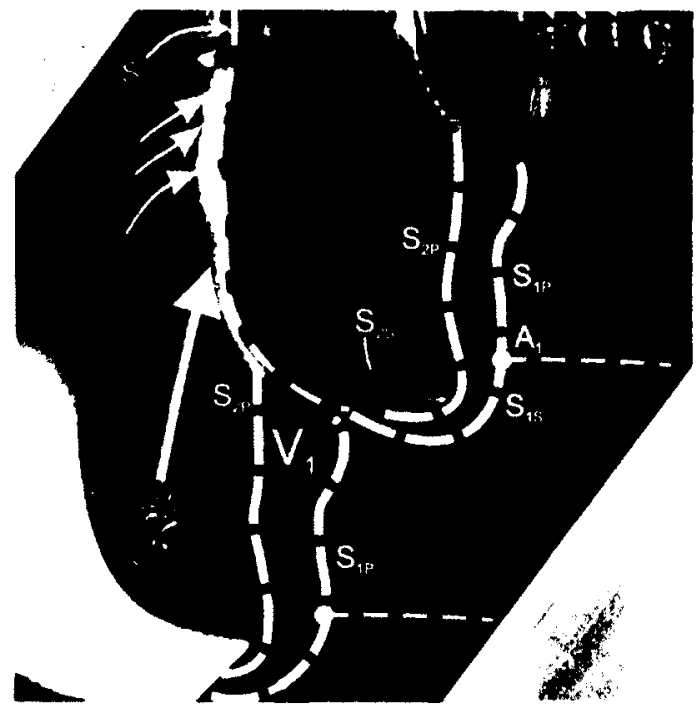

d)

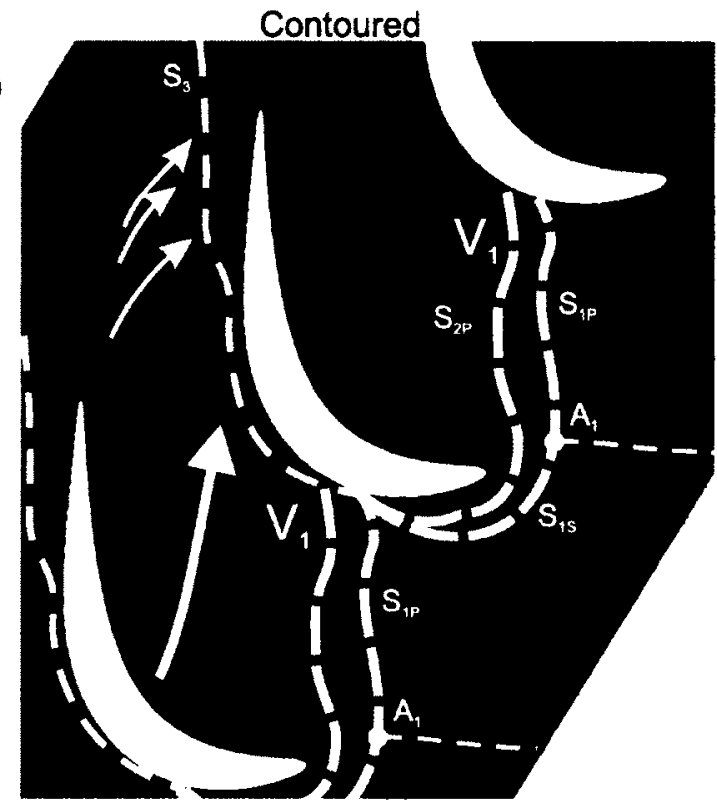

Figure 5.14: Comparison of experimental and predicted endwall flow visualizations for (a \& b) planar and (c \& d) contoured configurations

a lesser extent in Figure 5.14b for the contoured configuration as the cross flow is reduced.

The experimental flow visualization and pressure measurements presented above showed that the blending out of the contouring results in an increased pitchwise cross flow near the endwall in the aft portion of the blade passage. This is also seen in the simulations where the small arrows near the exit of the blade row indicate that the surface streamlines are 
turned more sharply toward the suction surface in Figure 5.14d than they are in Figure 5.14b. As in the experiment, the increased cross flow appears to intensify the corner vortex, and its increase in size is seen as the distance between $S_{3}$ and the suction surface grows. The extent to which this happens is over predicted by the simulations comparing the distance between $S_{3}$ and the suction surface of Figures $5.14 \mathrm{c}$ and $5.14 \mathrm{~d}$.

Figure 5.15 compares the predicted surface streamlines to the experimental results for the oil surface flow visualization on the suction surface of the airfoil. As was seen on the endwall above, the simulations capture the flow physics reasonably well. The twodimensional separation bubble bound by the $S_{5}$ separation and $R_{5}$ reattachment lines is evident in the computational results. The location of the laminar separation is well captured. However, the streamwise length of the separation bubble is under predicted suggesting that the transition process in the free shear layer occurs in a shorter streamwise distance in the simulations.

Examining the predicted surface streamlines between the $R_{3}$ reattachment $S_{4}$ separation lines of Figures $5.15 \mathrm{~b}$ and $5.15 \mathrm{c}$, it is seen that the streamlines are turned more toward midspan for the planar configuration. This is consistent with the discussion of the pressure probe results presented above which showed that the planar configuration sees stronger flow towards the midspan of the blade. Figure $5.13 \mathrm{c}$ shows a reduction in the blade loading static pressure coefficient $\left(C_{P-L}\right)$ at $10 \%$ span for the contoured configuration. In the two dimensional flow at midspan, the reduction in $C_{P-L}$ would result in less pitchwise flow turning. Here however, the decreased three-dimensionality of the flow results in more pitchwise flow turning despite $C_{P-L}$ being reduced by the contouring. 
a)

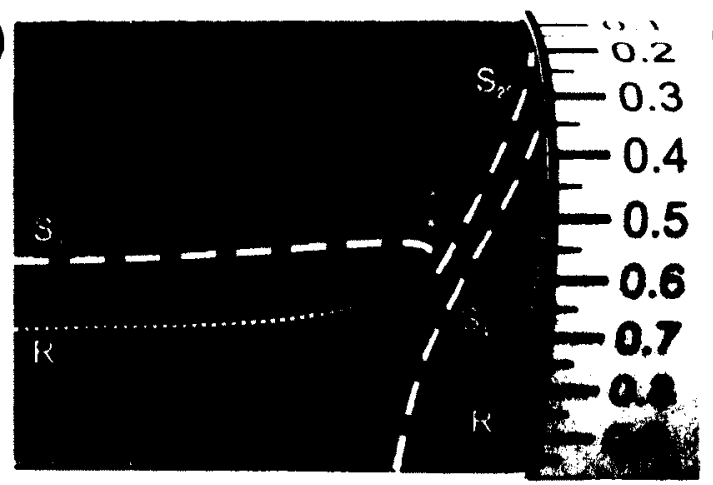

Planar

b)

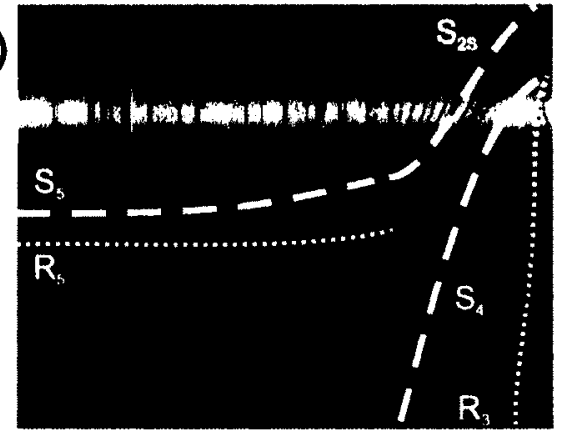

c)

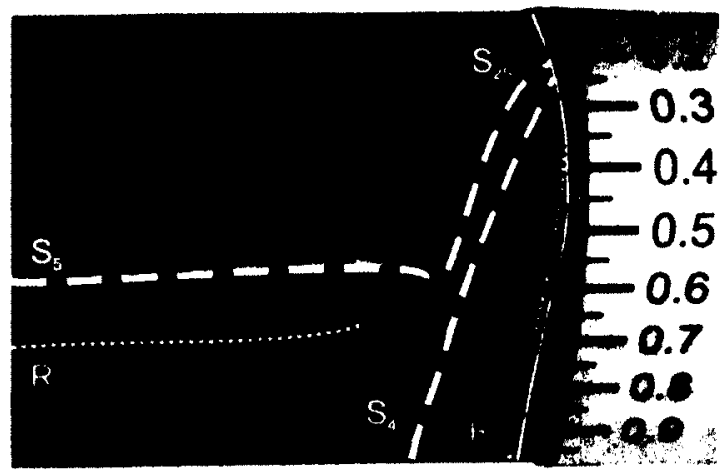

Contoured

d)

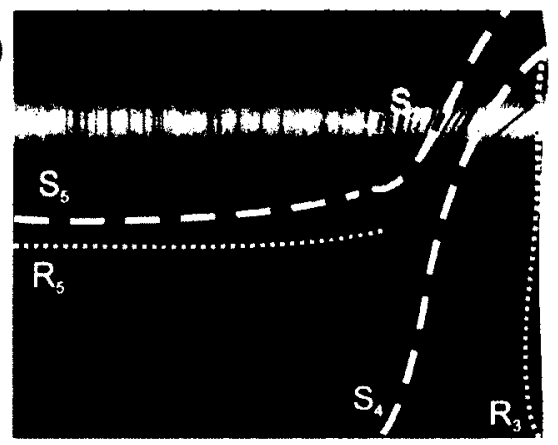

Figure 5.15: Comparison of experimental and predicted suction surface flow visualizations for (a \& b) planar and (c \& d) contoured endwall configurations

\subsubsection{Predicted Losses and Flow Physics}

The predicted and experimentally measured flow fields for the planar and contoured configurations are compared in detail in Figure 5.16. In general the results agree with the findings of the two and three-dimensional grid independence studies presented in the previous chapter. As was found in the grid independence studies, the peak loss for the various structures (two-dimensional wake, passage vortex etc.) is over predicted and their diffusion is under predicted. In free shear layers away from surfaces, the SST turbulence closure model reverts to a transformed version of the $k-\epsilon$ model by means of a blending function (Menter, 1994). The $k-\epsilon$ model's inability to capture the diffusion of a shear flow is well documented. For example, Wilcox (1993) examined the $k-\epsilon$ model for a variety of 
free shear flows and found that the predicted spreading rate of a two-dimensional far wake is $30 \%$ lower than measured. This is consistent with what is seen in the present simulations at midspan for both the contoured and planar configurations.

In addition, there is less mixing of the predicted flow structures. The results of these simulations show three cores of positive vorticity rather than the two that are measured experimentally or predicted in the grid independent results of Section 4.3.8. In Figures 5.16c and $5.16 \mathrm{~d}$ the strong cores of positive vorticity closest to and furthest from the endwall pertain to the corner vortex and the wall induced vortex (see Figure 5.6b), respectively. The third core of positive vorticity between them relates to vorticity generated along the suction surface of the blade as a result of the no slip condition at the blade surface and the shearing of the boundary layer in the spanwise direction away from the root by the passage vortex. Typically this vorticity combines with that of the wall induced vortex a short distance downstream of the trailing edge as in the PAKD-F simulations of Section 4.3.8.

The predicted and measured results at the exit plane are pitchwise mass-averaged in Figure 5.17. The simulations capture the trends seen in the experimental data quite well. The pitchwise mass-averaged loss coefficient (Figure 5.17a) sees a reduction in its peak magnitude. The location of peak loss is predicted to move closer to the endwall creating a larger region of two-dimensional flow at midspan. That being said, both the simulations for the planar and endwall configurations over predict the penetration of the secondary flow toward midspan and the magnitude of the loss relative to the measured data. Between the endwall and $8 \%$ span the predictions capture the fact that the contoured configuration generates higher losses. Overall, the magnitude of the secondary loss is over predicted. At midspan however, the transition model does an exemplary job in predicting the momentum deficit generated in the boundary layer. Traditionally this has been a challenge when predicting linear cascade flows using turbulence closure models that do not 
a)

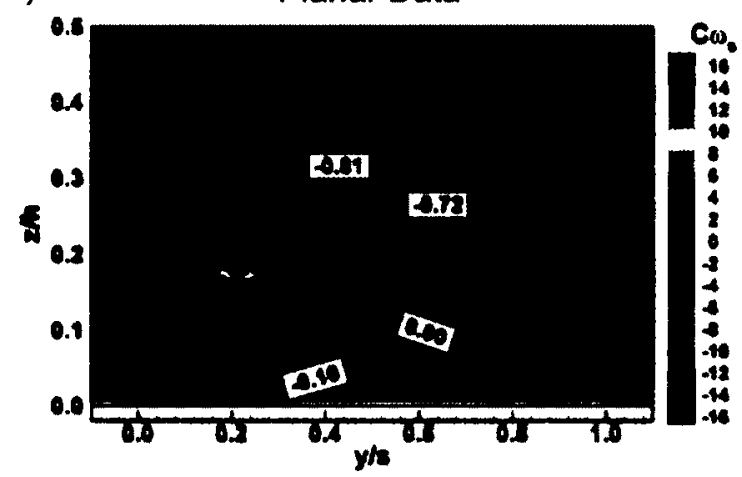

b)

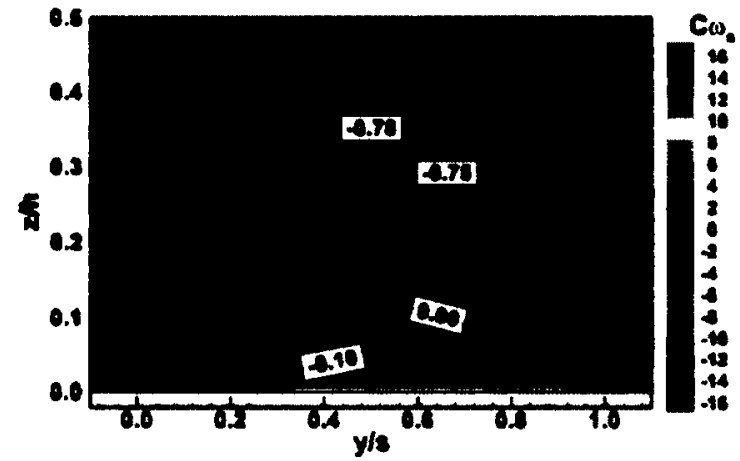

c)

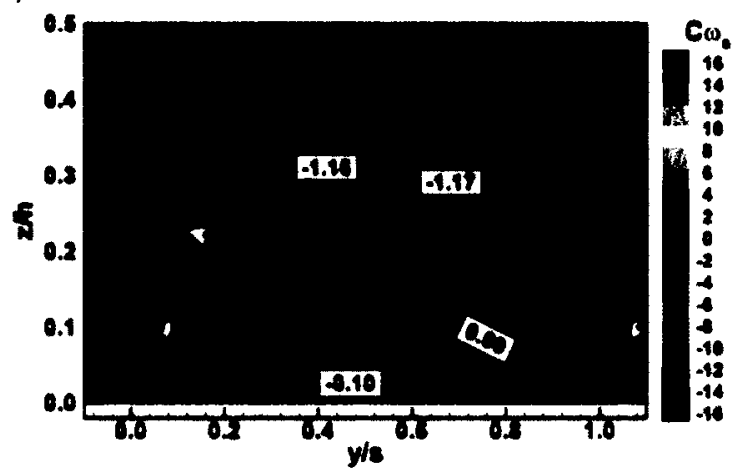

d)

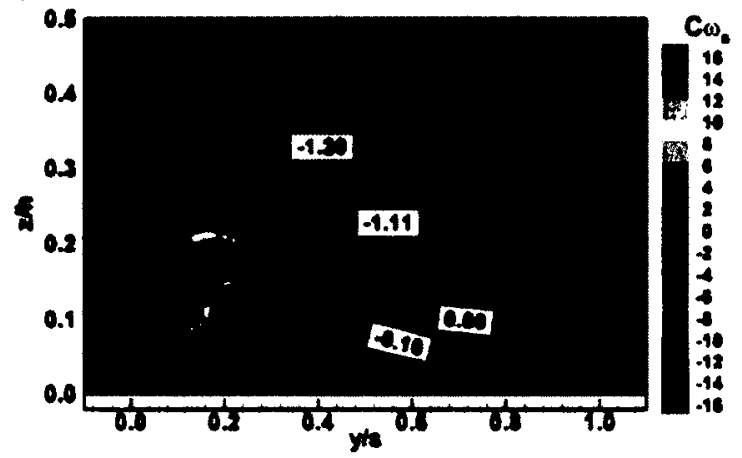

Figure 5.16: Measured and predicted secondary velocity vectors with flood of streamwise vorticity coefficient $\left(C_{\omega s}\right)$ (a \& c), and line contours of total pressure loss coefficient $\left(C_{P_{o}}\right)$ with flood of secondary kinetic energy coefficient $\left(C_{S K E}\right)$ (b \& d), at $140 \%$ axial cord

employ a transition model (Cleak and Gregory-Smith, 1992; Hartland et al., 2000)

The variation of the pitchwise flow angle resulting from the application of endwall profiling is also captured by the simulations (Figure $5.17 \mathrm{~b}$ ). The overturning near the endwall is predicted to increase and the under turning resulting from the passage vortex to decrease. As with the predicted pressure losses, the peak magnitude of the pitchwise flow angle is larger than was measured experimentally.

The computations also capture the change of the secondary kinetic energy coefficient with the application of endwall contouring (Figure 5.17c). Like the experimental measurements, the predictions show that the $C_{S K E}$ associated with the secondary flow is reduced 

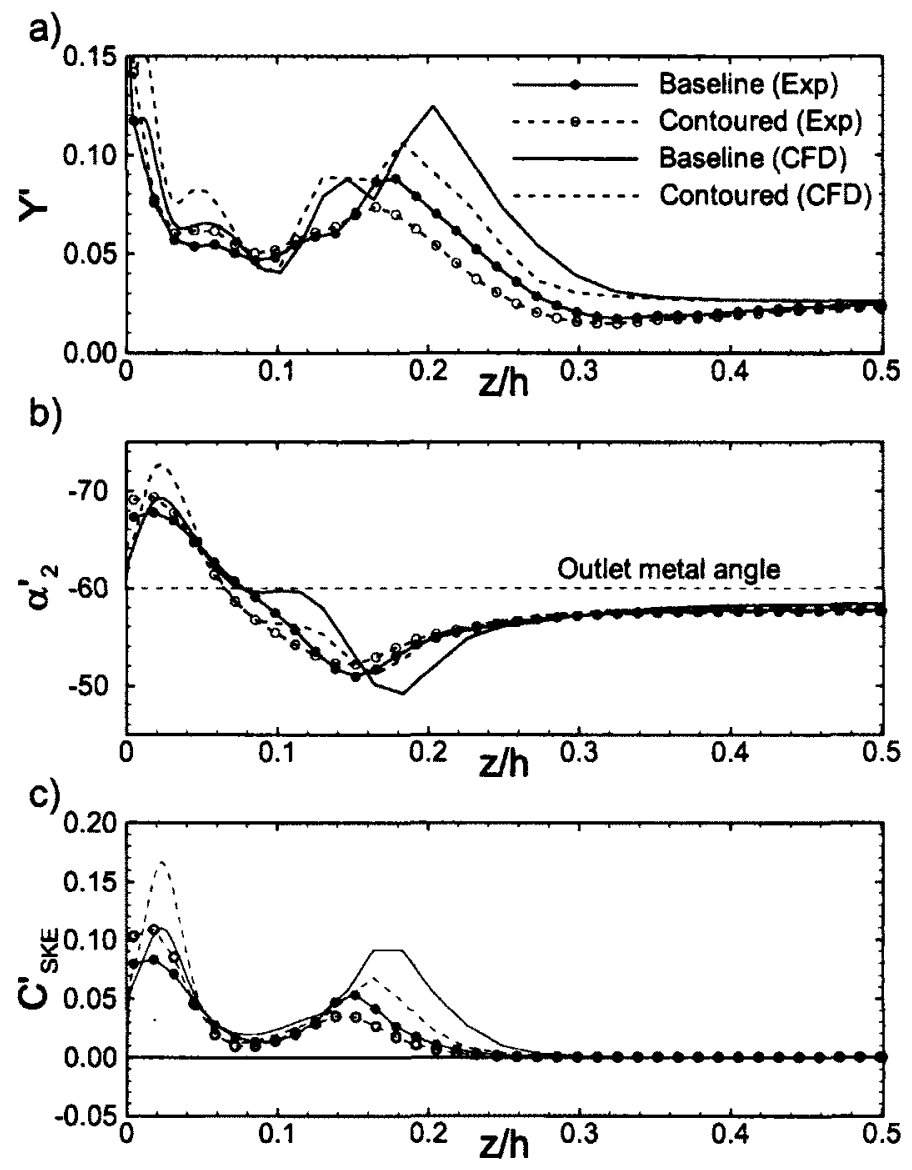

Figure 5.17: Measured and predicted pitchwise mass-averaged results for the $140 \% B_{x}$ plane; Total pressure loss coefficient (a), outlet flow angle (b) and secondary kinetic energy coefficient (c)

by profiling the endwall. Furthermore, near the endwall the contouring generates higher secondary kinetic energy due to the increased pitchwise over turning of the flow. As above, the magnitude of the secondary kinetic energy is over predicted suggesting that the secondary velocities generated within the blade passage are being dissipated too slowly by the turbulence model. This has been observed by other researchers (eg. Cleak and Gregory-Smith, 1992).

By and large the pitchwise mass-averaged comparisons of the predicted and measured results in the current study are in good agreement with those presented by Hartland et al. 
(2000) for the evaluation of their contoured endwall relative to the planar configuration. The simulations of Hartland et al. (2000) used a structured grid topology and an algebraic mixing length turbulence model with a wall function. Like the simulations in the current study, the predictions of Hartland et al. (2000) showed a reduction in size of the secondary flow structure, a reduction in loss associated with the secondary flow, and somewhat higher losses near the endwall likely resulting from less convection of higher loss fluid.

The comparison of the measured and predicted results at the exit plane for the current study are summarized in Table 5.2. The values in the table represent percent changes relative to the planar case.

Table 5.2: Effect of endwall contouring at $1.40 B_{x}$, change in measured mass-averaged quantities relative to planar case

\begin{tabular}{lcr} 
Flow Quantity & Measured & Predicted \\
\hline \hline$\Delta C_{P o_{\text {total }}}$ & $-7.1 \%$ & $-8.5 \%$ \\
$\Delta C_{P o_{\text {secondary }}}$ & $-9.8 \%$ & $-21.1 \%$ \\
$\Delta C_{S K E}$ & $-13.3 \%$ & $-23.7 \%$ \\
$\Delta Y_{\text {total }}$ & $-9.3 \%$ & $-9.0 \%$ \\
$\Delta Y_{\text {secondary }}$ & $-15.8 \%$ & $-21.3 \%$ \\
\hline \hline
\end{tabular}

Despite the shortcomings noted in this subsection, the simulations generated a satisfactory prediction of the change in blade row loss $\left(\Delta C_{P o_{\text {total }}}\right.$ and $\left.\Delta Y_{\text {total }}\right)$. Since the predicted change in secondary kinetic energy is larger than that measured experimentally, the simulation for the contoured configuration has stronger primary kinetic energy at the outlet and thus slightly lower $\Delta Y_{\text {total }}$.

The simulations produce trend accurate predictions of the change in blade row loss; however, it is evident that the change in secondary kinetic energy and change in secondary loss is over estimated by approximately a factor of two. It is not surprising that the computations fail to accurately predict the secondary flows in a turbine blade passage. All two-equation turbulence models use closure coefficients to replace the unknown double 
and triple correlations of fluctuating velocity, pressure, and velocity gradients. Near solid boundaries, where the no slip condition is applied, the Shear Stress Transport (SST) model used in the present simulations applies the $k-\omega$ formulation of Wilcox (1993). The model closure coefficients are established assuring similitude with the observed properties of turbulence by examining decaying homogeneous and isotropic turbulence, the log law layer and the defect layer. From experience, the $k-\omega$ model is known for its ability to predict two-dimensional boundary layers with favourable and adverse pressure gradients. It is also successful at predicting two-dimensional separation and reattachment points. By its definition, it is less satisfactory for computing the boundary layers the endwall region which are dominated by skew and three-dimensional separations. Away from solid boundaries, the SST model switches to a transformed version of the $k-\epsilon$ model. The original closure coefficients of Jones and Launder (1972) (tuned for attached two-dimensional boundary layers with favourable pressure gradients) were reevaluated by Launder and Sharma (1974) to incorporate the high gradients of swirl velocity in the vicinity of a rotating disc in a quiescent atmosphere. Thus the $k-\epsilon$ model is not apt for predicting diffusion and dissipation in the free stream of a turbine cascade secondary flow which is dominated by vortical shear and non-isotropic turbulence. This being said, the SST model offers a reasonable engineering solution to the prediction of secondary flows and the trend accurate results appear to be a reasonable basis for the design and optimization of endwall contouring.

\subsection{Conclusions}

Experimental results have been presented documenting changes to the flow field as a result of the application of non-axisymmetric endwall contouring. In broad terms, the results confirm what has been shown by a number of earlier researchers; endwall contouring is an effective means of reducing endwall losses. In addition to this, a number of insights 
and contributions have been gained throughout the present work:

Endwall contouring reduces the strength of pitchwise cross-passage flow near the endwall and spanwise flow up the suction surface of the blade, resulting in a weaker passage vortex. The weaker passage vortex delays the roll up of the secondary flow and reduces the amount of fluid convected into it.

This chapter has also put forth a detailed interpretation of the three dimensional flow field within the blade passage of a PAKB linear cascade, further expanding the database for this established research airfoil. The interpretation is based upon oil surface flow visualizations and a series of intra-passage seven-hole pressure probe measurements.

The complementary computational analysis has shown that a RANS based approach employing the SST turbulence model coupled with the Gamma-Theta transition model can predict the flows of a linear cascade of low-pressure turbine blades. The variations in flow physics resulting from the application of non-axisymmetric endwall contouring are captured to a large extent and the predicted variation in total pressure loss is predicted with trend accuracy.

Finally, the results presented herein lay the ground work for subsequent chapters investigating secondary loss reduction for more highly-loaded turbine airfoils. Following chapters will examine the effect of overall loading level and loading distribution on loss reduction mechanisms and build on the discussion put forth. 


\title{
Chapter 6
}

\section{Secondary Flow in a High-Lift,}

\section{Front-Loaded Turbine Cascade}

\author{
with the Implementation of
}

Non-Axisymmetric Endwall

\section{Contouring}

\subsection{Introduction}

This is the second chapter studying the mitigation of endwall losses using nonaxisymmetric endwall contouring. The previous chapter documented the baseline test case for the study. The current work investigates the secondary flow in a cascade of more highly-loaded low-pressure turbine airfoils with and without the implementation of endwall profiling. As noted earlier, this study is novel in two regards. First, the contouring is 
applied to low-pressure turbine airfoils, whereas studies conducted by other researchers have focused their endwall profiling efforts on the high-pressure turbine. Second, while previous researchers have optimized contouring designs for a given airfoil, the current work demonstrates the potential to open the design space by employing high-lift airfoils in conjunction with endwall contouring. The previous chapter investigated the effectiveness of endwall profiling on a baseline cascade of PAKB airfoils, having a Zweifel coefficient $\left(Z_{w}\right)$ of 1.08. The current chapter evaluates the effectiveness of endwall contouring on the high-lift forward-loaded PAKD-F cascade having approximately $25 \%$ higher loading $\left(Z_{w}=1.36\right)$.

\subsection{Motivation}

The rising cost of fuel has placed the burden on aero engine designers to create light weight, highly efficient low-pressure turbines. The low-pressure turbine is an obvious candidate for a weight reduction program as it can account for as much as a third of the engine's total mass Curtis et al. (1997). Engine weight can be decreased by reducing the number of blades per stage thereby increasing the aerodynamic duty of each blade. It is commonly accepted that higher secondary losses are inherent to increased blade loading, thus rendering highly loaded stages unattractive from an efficiency stand point. In recent years non-axisymmetric endwall contouring has been shown as an effective means to reduce endwall losses. With this development, the possibility arises to increase blade loading and avoid the associated secondary loss.

Secondary losses in axial flow turbines have been found to be sensitive to blade loading. Hodson and Dominy (1987a), and Perdichizzi and Dossena (1993) both varied the pitch to chord ratio in high speed linear cascade wind tunnels. Perdichizzi and Dossena (1993) simply increased the pitch spacing of the cascade while Hodson and Dominy (1987a) varied the stagger angle as well to maintain the exit flow angle. The results of both studies were 
somewhat clouded by changes to the suction surface boundary layer, but several trends emerged. Increasing the pitch-chord ratio resulted in higher blade loading, larger variations in pitchwise over/underturning, higher secondary kinetic energy, and higher secondary loss.

Loading distribution also plays an important role in secondary loss generation. Weiss and Fottner (1995) tested the front-loaded T104 and aft-loaded T106 in a high speed linear cascade wind tunnel. At the furthest downstream measurement plane the front-loaded airfoil produced higher secondary losses and higher secondary kinetic energy.

More closely related to the current work, Zoric et al. (2007a) presented profile and secondary loss data for the PAK family of low-pressure turbine airfoils tested in Carleton University's low-speed linear cascade wind tunnel. At the time the family consisted of three airfoils having the same velocity triangles; the baseline airfoil is designated PAKB, and two others having $25 \%$ higher loading, with the aft-loaded designated PAKD-A and the front-loaded designated PAKD-F. In agreement with the studies above (Hodson and Dominy, 1987a; Perdichizzi and Dossena, 1993; Weiss and Fottner, 1995), the higher loaded cascades suffered a performance penalty, with the front-loaded PAKD-F producing the highest row loss and secondary kinetic energy.

Previous to the study of Zorić et al. (2007a), Popovic et al. (2006) tested the midspan performance of the PAK family over a range of freestream turbulence intensity (FSTI) and Reynolds number. They found that PAKD-F has exceptionally good midspan behaviour, matching the profile loss performance for the more lightly-loaded baseline airfoil at the elevated level of FSTI over the range of Reynolds number tested. PAKD-A on the other hand was found to have poor midspan performance and stalled at a Reynolds number just below the design value of 80,000 . More recently Praisner et al. (2008) showed midspan results for the expanded PAK family of airfoils. Two new airfoils were included in the study having $42 \%$ (PAKE) and $61 \%$ (PAKF) higher loading than the baseline PAKB airfoil. These 
"next generation" airfoils had the highest loading levels in the open literature at the time of publication. Their loading distributions were relatively front-loaded when compared to PAKB. PAKF, the highest loaded in the family had comparable midspan performance to the baseline airfoil over the range of Reynolds numbers tested.

The results of Zorić et al. (2007a), Popovic et al. (2006) and Praisner et al. (2008) show that the current state of the art design tools are capable of generating high-lift front-loaded airfoils that have good midspan performance, but suffer from relatively high endwall losses. As such, these designs are prime candidates for the implementation of non-axisymmetric endwall contouring to reduce secondary loss.

Optimization methods for endwall contours were presented by Yan et al. (1990) and Harvey et al. (2000), who used simulations employing an algebraic mixing-length model. Both methods used secondary kinetic energy as their optimization criterion based on the opinion that accurately predicting total pressure loss was unachievable with a mixing length turbulence closure model. The contoured endwall for the current study was generated with the method of Praisner et al. (2007). The technique used a sequential quadratic programming gradient-based algorithm with mass-averaged exit plane total-pressure loss as the objective function. Gradient searches were conducted for approximately 1000 design iterations, preformed with a RANS code using the $k-\omega$ turbulence model; the authors found trend accurate predictions of secondary loss reduction in comparison to experimental results.

Experimental investigations in low-speed linear cascades have confirmed that endwall contouring can successfully reduce secondary losses. Studies such as Hartland et al. (2000), Ingram et al. (2002) and those presented in the Chapter 5 have investigated blade rows more lightly-loaded than what is tested in the current chapter. These studies have shown a performance benefit can be achieved through the reduction in secondary kinetic energy that leads to reduced rollup of the passage vortex. Engine representative tests were performed 
by Rose et al. (2001), who described the redesign of a high-pressure turbine stage in a model rig. They found that the stage efficiency could be improved by $0.4 \%$ by contouring the nozzle guide vane and the first stage rotor.

\subsection{Approach}

The current chapter examines the secondary flows of the high-lift front-loaded lowpressure turbine cascade designated PAKD-F, with and without the implementation of endwall contouring. The discussion is focused on the physics of the endwall flow within the blade passage and downstream of the trailing edge. The study builds on the baseline test case documented in Chapter 5 by demonstrating the potential to expand the design space for high-lift airfoils by applying endwall contouring.

The cascade was shown in Figure 3.4 and the geometric details were given in Table 3.2. The testing was conducted at an inlet Reynolds number of 126,000 base on axial chord and at design incidence.

\subsection{Endwall Contouring}

The cascade consists of seven PAKD-F airfoils. Measurements were taken for the fourth blade passage from the top of the cascade. The test section was converted to the profiled endwall configuration by replacing the planar insert which mounts the blades on the turntable with one that is contoured. All six blade passages were contoured to ensure periodic flow at the outlet of the cascade. A rendering of the contoured endwall is presented in Figure 6.1. The contouring consists of a hill $(H)$, and two valleys $\left(V_{1}\right.$ and $\left.V_{2}\right)$ constrained to be within the blade passage. The maximum and minimum amplitudes of the profiling are $10.1 \% B_{x}$ into the flow and $7.2 \% B_{x}$ into the endwall. The topology of the contoured 
endwall is comparable to that presented in the previous chapter.

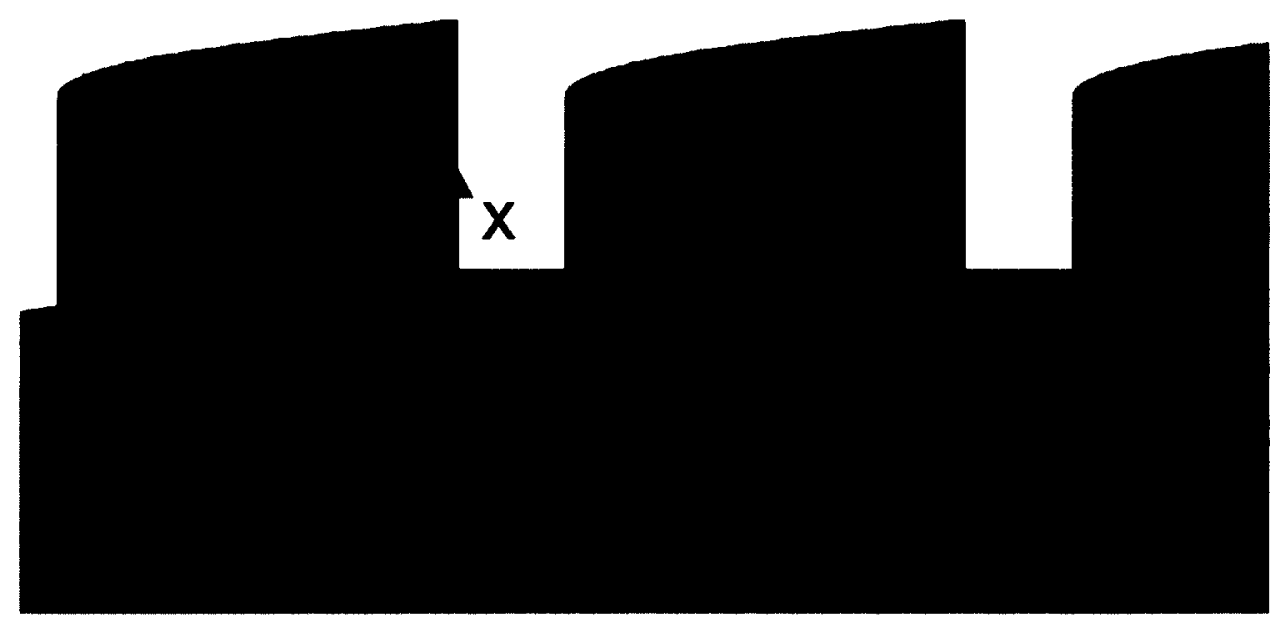

Figure 6.1: Isometric view of PAKD-F profiled endwall

\subsection{Results and Discussion}

\subsubsection{Cascade Inlet Conditions}

As documented by Section 5.5.1, the turbulence generating grid produced a total pressure non-uniformity to occur over a significant spanwise distance upstream of the cascade. Some concern arose regarding the variation of inlet boundary layer parameters across the pitch of the blade passage. As a result, three traverses were performed at $1.2 B_{x}$ upstream of the leading edge of the cascade at midpitch and $25 \%$ blade spacing to either side. The results of the pitot tube traverses, shown in Figure 6.2, suggest that the boundary layer is relatively uniform in the pitchwise direction. The range of integrated boundary layer parameters for the three traverses is presented in Table 6.1. The boundary layer shape factor $(H)$ is typical of a turbulent boundary layer with zero pressure gradient. To account for the non-uniformities caused by the grid, the inlet was traversed with a three-hole pressure probe 
over one blade pitch from the wall to half span; the total pressure was mass-averaged as the reference inlet total pressure coefficient $\left(C_{P o, 1}^{\prime \prime}\right)$. At midspan, the variation in total pressure coefficient and flow angle is within probe measurement error across four blade pitches.

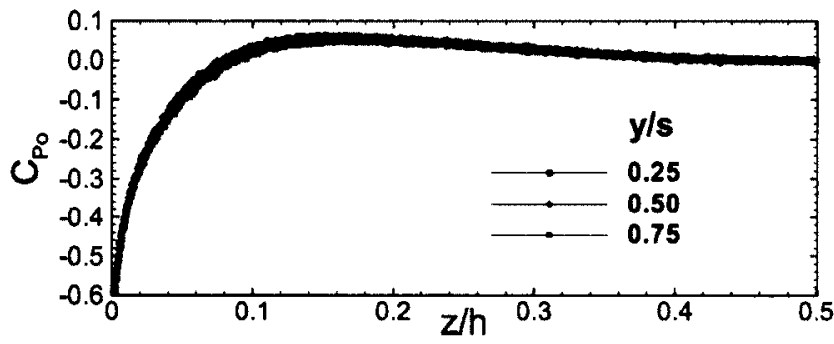

Figure 6.2: Spanwise variation of total pressure coefficient as measured with a pitot probe $120 \%$ axial chord upstream of the leading edge at $y / s=0.25,0.5$ and $\mathbf{0 . 7 5}$

\subsubsection{Pressure Probe Results}

Seven hole pneumatic probe measurements were made on two axial planes within the blade passage, at the trailing edge plane, and at two downstream planes.

The results for the $0.63 B_{x}$ plane are presented in Figure 6.3. The figure contains two different types of plots showing the results for the planar and contoured endwall side by side. The view is looking upstream with the passage suction side on the left and the pressure side on the right. In the first type of plot, a flood of negative streamwise vorticity coefficient $\left(C_{\omega s}\right)$ is superimposed with secondary velocity vectors for the planar and contoured endwall, in Figures $6.3 \mathrm{a}$ and $6.3 \mathrm{c}$, respectively. The secondary velocity vectors show the components of velocity on a plane normal to the mass-averaged flow velocity vector. For the right hand coordinate system selected, the clockwise rotating passage vortex has negative vorticity. These plots enable the passage vortex to be tracked in subsequent figures since streamwise vorticity is convected with fluid particles. 
Table 6.1: Summary of PAKD-F results

\begin{tabular}{|c|c|c|c|}
\hline \multirow[b]{2}{*}{ LOCATION } & \multirow[b]{2}{*}{ FLOW QUANTITY } & \multicolumn{2}{|c|}{ ENDWALL } \\
\hline & & PLANAR & CONTOURED \\
\hline \multirow[t]{5}{*}{ INLET } & $\delta / h$ & \multicolumn{2}{|c|}{$0.16-0.16$} \\
\hline & $\delta^{*}[\mathrm{~mm}]$ & \multicolumn{2}{|c|}{$2.0-2.3$} \\
\hline & $\theta[\mathrm{mm}]$ & \multicolumn{2}{|c|}{$1.7-1.9$} \\
\hline & $H$ & \multicolumn{2}{|c|}{$1.20-1.21$} \\
\hline & $C_{P_{0,1}}^{\prime \prime}$ & \multicolumn{2}{|c|}{-0.0027} \\
\hline OUTLET & $C_{P o_{\text {total }}}$ & -0.150 & -0.127 \\
\hline \multirow[t]{12}{*}{$B_{x}=1.40$} & $C_{P o_{\text {profile }}}$ & -0.067 & -0.068 \\
\hline & $C_{P o_{\text {secondary }}}$ & -0.083 & -0.059 \\
\hline & $Y_{\text {total }}$ & 0.063 & 0.054 \\
\hline & $Y_{\text {profile }}$ & 0.028 & 0.029 \\
\hline & $Y_{\text {secondary }}$ & 0.035 & 0.025 \\
\hline & $C_{S K E}^{\prime \prime}$ & 0.031 & 0.020 \\
\hline & $\alpha_{2}^{\prime \prime}[\mathrm{deg}]$ & -56.8 & -57.0 \\
\hline & $\alpha_{2, \text { midspan }}^{\prime}[\mathrm{deg}]$ & -57.9 & -57.8 \\
\hline & $q_{2}^{\prime \prime} / q_{C L_{1}}$ & 2.334 & 2.324 \\
\hline & $q_{2, \text { midspan }}^{\prime} / q_{C L_{1}}$ & 2.359 & 2.345 \\
\hline & $A V R$ & 0.996 & 0.995 \\
\hline & $F_{Y}$ & 4.41 & 4.42 \\
\hline \multirow[t]{10}{*}{ MIXED-OUT } & $\overline{C_{P o_{\text {total }}}}$ & -0.188 & -0.154 \\
\hline & $C_{P o_{\text {profile }}}$ & -0.075 & -0.076 \\
\hline & $C_{P O_{\text {secondary }}}$ & -0.113 & -0.033 \\
\hline & $Y_{\text {total }}$ & 0.082 & 0.067 \\
\hline & $Y_{\text {profile }}$ & 0.032 & 0.033 \\
\hline & $Y_{\text {secondary }}$ & 0.050 & 0.034 \\
\hline & $\alpha_{2}^{\prime \prime}[\mathrm{deg}]$ & -57.7 & -57.8 \\
\hline & $\alpha_{2, \text { midspan }}^{\prime}[\mathrm{deg}]$ & -58.0 & -57.9 \\
\hline & $q_{2}^{\prime \prime} / q_{C L_{1}}$ & 2.259 & 2.281 \\
\hline & $q_{2, \operatorname{midspan}}^{\prime} / q_{C L_{1}}$ & 2.352 & 2.339 \\
\hline
\end{tabular}

Figure 6.3a shows a well defined peak of negative vorticity coefficient that lies below and to the left of the point about which the secondary velocity vectors revolve. On the other hand, the contoured endwall shows no defined peak of negative vorticity in Figure 6.3c. Also noteworthy is the fact that the point that the secondary velocity vectors appear to revolve about is $12 \%$ of pitch closer to the suction surface of the passage for the planar endwall. Thus, it seems that the convection of the passage vortex across the passage is 
delayed by the contouring. Hartland et al. (2000) and the results presented in Section 5.5.4 both showed a delayed migration of the passage vortex toward the suction surface as a result of the application of endwall contouring.
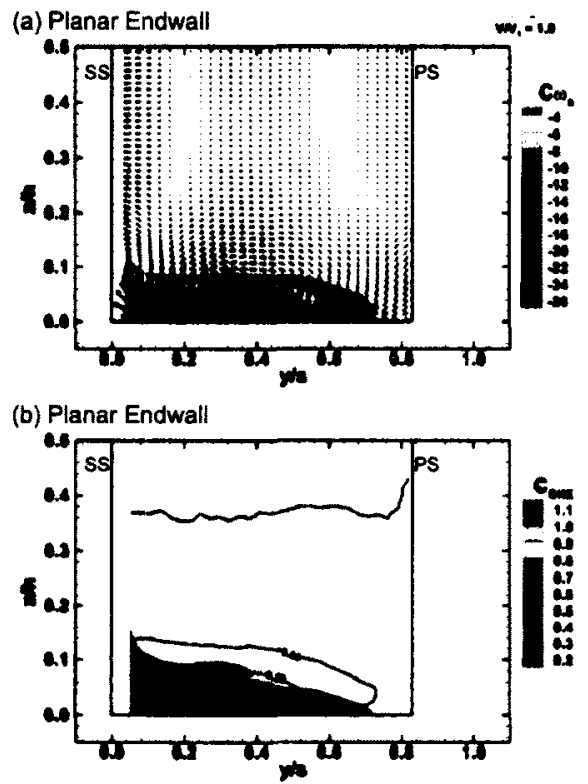

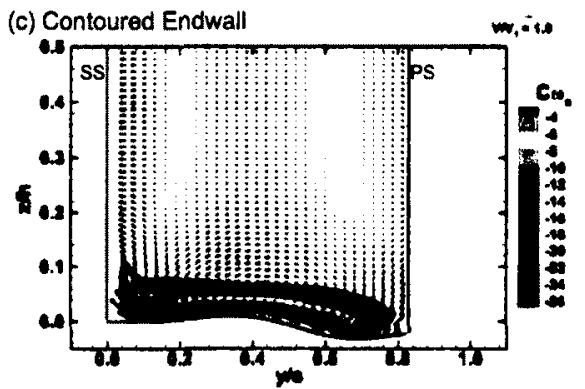

(d) Contoured Endwall

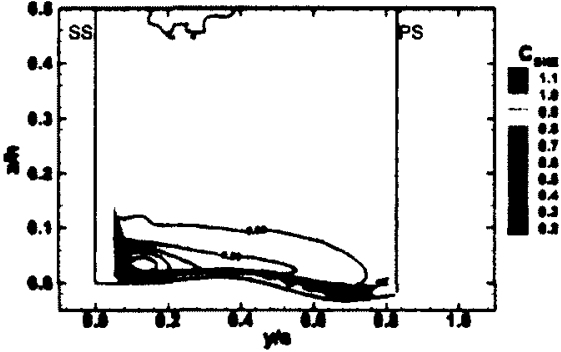

Figure 6.3: Secondary velocity vectors with flood of streamwise vorticity coefficient $\left(C_{\omega s}\right)$ (a \& $\left.\mathbf{c}\right)$, and line contours of total pressure loss coefficient $\left(C_{P_{o}}\right)$ with flood of secondary kinetic energy coefficient $\left(C_{S K E}\right)(\mathrm{b} \& \mathrm{~d})$, at $63 \%$ axial cord

The second type of plot in Figure 6.3 shows a flood of secondary kinetic energy coefficient $\left(C_{S K E}\right)$ overlaid with line contours of total pressure loss coefficient $\left(C_{P o}\right)$. The secondary kinetic energy coefficient is the kinetic energy associated with the secondary velocity vectors normalized by the kinetic energy on the inlet centerline of the cascade. Since $C_{S K E}$ is a function of the square of the secondary velocity, it emphasizes subtle differences in the magnitude of the secondary velocity vectors. In this case, the strength of the cross-flow, as measured by the $C_{S K E}$ near the endwall is accentuated, and is roughly twice as strong for the planar endwall case. It is believed that the cross-flow into the suction-surface/endwall junction is responsible for the roll-up of the passage vortex (Hartland et al., 2000; Ingram et al., 2002; Section 5.5.4). The roll-up process appears more advanced for the planar 
case judging from the line contours of $C_{P o}$ in Figure 6.3b relative to those in Figure 6.3d. The core of peak loss in Figure $6.3 \mathrm{~b}$ associated with the passage vortex is being displaced away from the endwall by higher energy fluid being convected from the pressure side of the passage. In light of this, it could be argued that the planar endwall had stronger endwall cross-flow upstream of this measurement plane. In contrast, the contoured endwall does not exhibit a core of peak loss associated with the passage vortex, and likely had weaker cross-flow upstream of $63 \% B_{x}$.

Figure 6.4 presents the results for the $80 \%$ axial chord plane. The negative vorticity coefficient contours of Figure $6.4 \mathrm{a}$ once again show a peak that nearly coincides with the core of the passage vortex. The location of the core has moved about $4 \%$ of pitch toward the suction surface relative to its position in Figure $6.3 \mathrm{a}$. The migration of the passage vortex toward the suction surface and away from the endwall has been seen previously by other researchers for a planar endwall case, most notably perhaps by Gregory-Smith and Graves (1983). The rate of migration and the final position of the passage vortex has been linked to the strength of the secondary flow. The vorticity flood contour plot of Figure $6.4 \mathrm{c}$ shows that the contouring reduces the strength of the passage vortex relative to the planar endwall. The negative vorticity appears to be split between two cores, one centred at $y / s=0.1$ and another at $y / s=0.45$. The contoured endwall of Hartland et al. (2000) also seemed to split the passage vortex into two discrete vortices near the exit of the blade row.

The secondary kinetic energy coefficient flood of Figure $6.4 \mathrm{~b}$ reveals two peaks. The first, close to the endwall is associated with the pitchwise cross-flow toward the suction surface. The second, near the suction surface is associated with spanwise flow away from the endwall. The line contours of total pressure loss again show a defined loss core linked to the passage vortex. The core appears to have diffused relative to that of Figure $6.3 \mathrm{~b}$ and the peak loss has dropped by roughly $20 \%$. A high loss region exists near the suction-surface/endwall 

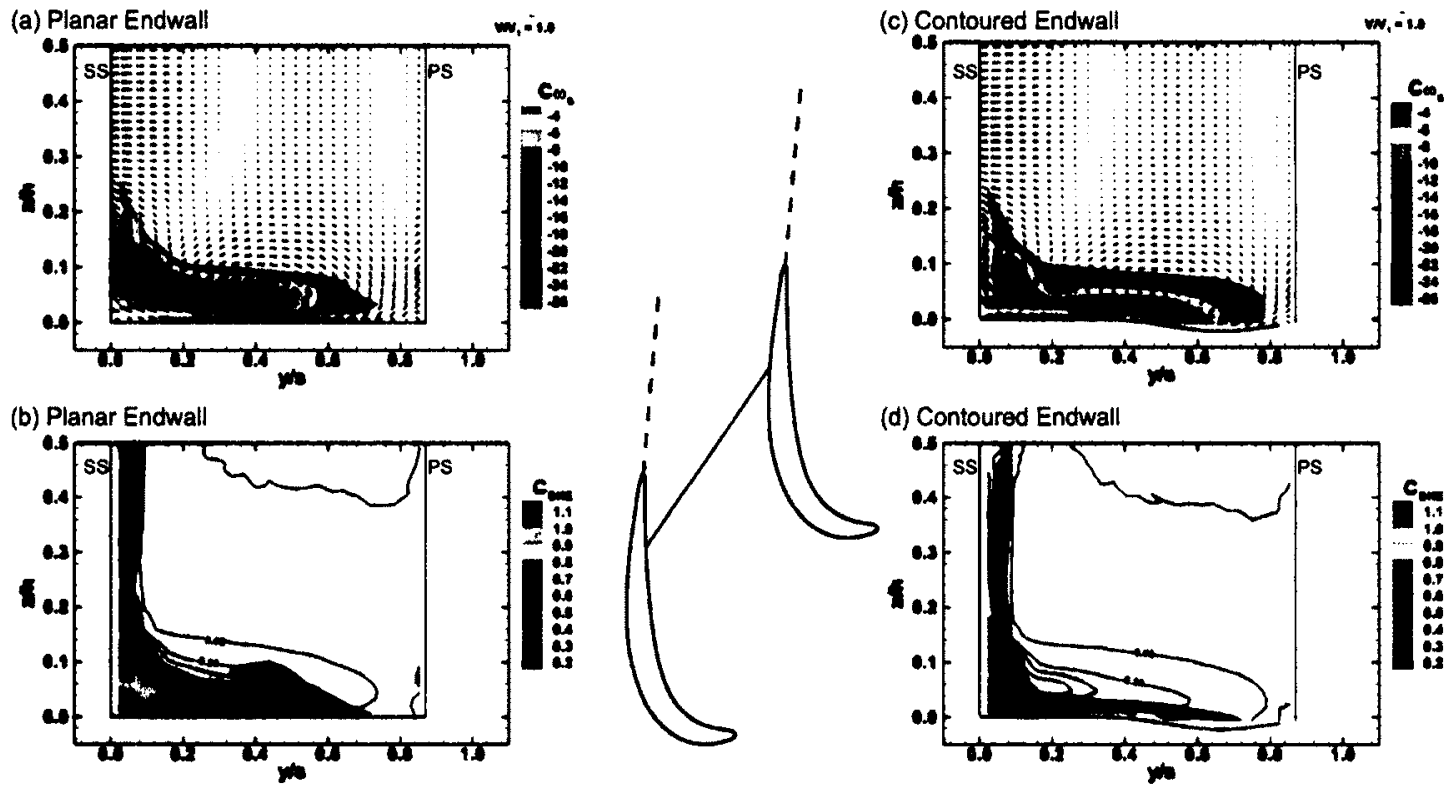

(d) Contoured Endwall

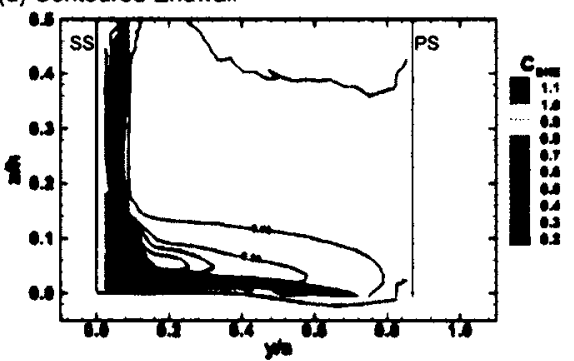

Figure 6.4: Secondary velocity vectors with flood of streamwise vorticity coefficient $\left(C_{\omega s}\right)(\mathbf{a} \& \mathbf{c})$, and line contours of total pressure loss coefficient $\left(C_{P o}\right)$ with flood of secondary kinetic energy coefficient $\left(C_{S K E}\right)$ (b \& d), at $80 \%$ axial cord

junction between zero and $20 \%$ pitch and up to $3 \%$ span. This loss core is attributed to the corner vortex which is comprised of fluid having positive vorticity. Between the corner and passage vortices, higher energy fluid appears to be convected from the pressure side of the passage and is displacing the suction surface boundary layer. Figure $6.4 \mathrm{~d}$ shows that the contoured endwall generates weaker cross-passage and spanwise flow as determined by the $C_{S K E}$. In the previous chapter it was proposed that the reduced convection of fluid away from the endwall, up the span of the blade, was important to the reduction of secondary losses for the contoured endwall tested.

By the trailing edge plane, the contouring blends out to the nominal endwall height in the direction of the outlet metal angle. This affects the flow field by increasing the overturning near the endwall; Figures $6.5 \mathrm{~b}$ and $6.5 \mathrm{~d}$ show that the strength of the cross flow for the contoured case is nearly the same as that of the planar endwall. The planar 
endwall, however, continues to have higher secondary kinetic energy associated with the spanwise flow near the suction surface. The line contours of total pressure loss for the planar endwall continue to show a pattern typical of a linear cascade. The loss core related to the passage vortex consists of fluid having the highest negative vorticity. This same loss core is not evident in the line contours of Figure $6.5 \mathrm{~d}$. Also worth mentioning is that the suction surface boundary layers of both configurations are being displaced away from the endwall by higher energy fluid convected from the pressure side of the passage. Suction surface boundary layer fluid also appears to be induced away from the blade and is entrained around the passage vortex. Both processes seem to be more advanced for the planar case.
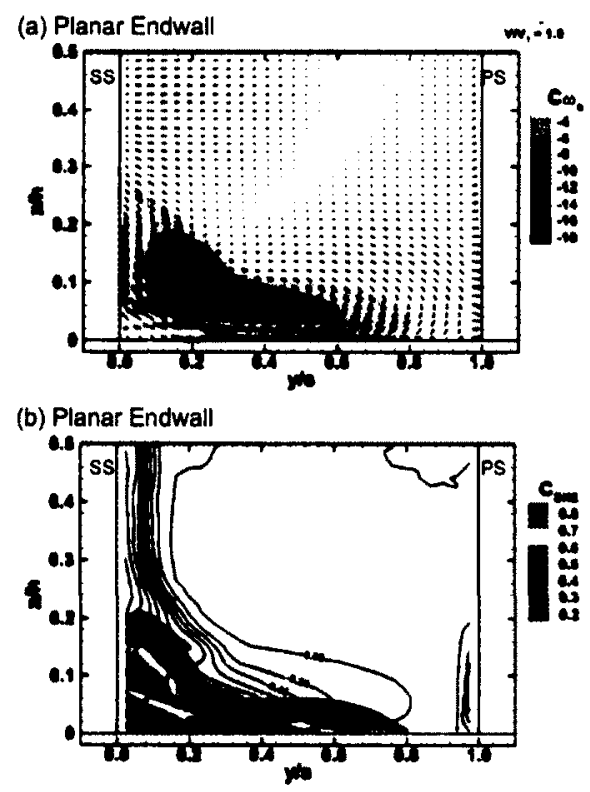
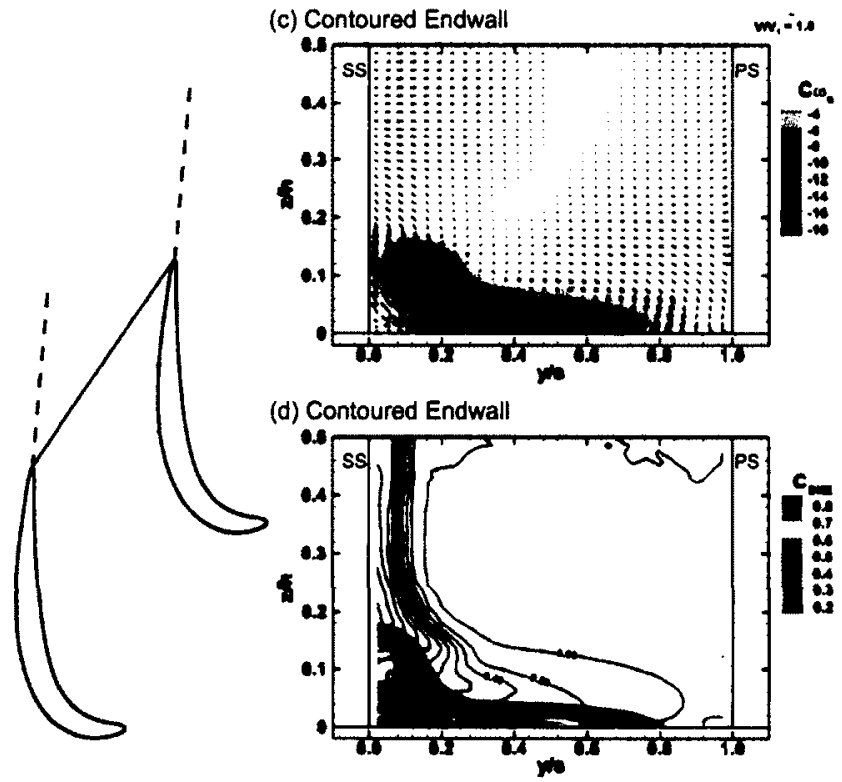

Figure 6.5: Secondary velocity vectors with flood of streamwise vorticity coefficient $\left(C_{\omega s}\right)(\mathbf{a} \& \mathbf{c})$, and line contours of total pressure loss coefficient $\left(C_{P_{0}}\right)$ with flood of secondary kinetic energy coefficient $\left(C_{S K E}\right)(\mathrm{b} \& \mathrm{~d})$, at the trailing edge plane

The corner vortex, which was barely visible upstream in Figure 6.4d has grown to a size and strength almost that of the planar case in Figure $6.5 \mathrm{~b}$ in a short axial distance. The flow visualization and pressure probe results of Hartland et al. (2000), and those of the 
previous chapter showed that as the contouring blends out, the corner vortex is energized by the increased cross-flow. In a planar cascade, Hodson and Dominy (1987a) saw a similar link between the strength of the cross-flow and the corner vortex when the pitch to chord ratio of the cascade was increased.

The floods of streamwise vorticity coefficient show much the same trends that developed at the upstream measurement plane. The core of peak vorticity for the planar case (Figure 6.5a) continues to move towards the suction surface and away from the endwall. In doing so, it diffuses further and the maximum value of vorticity decreases relative to the upstream plane. The contoured case again appears to have its negative vorticity split between two focal points, one closer to the suction surface at $y / s=0.12$ and one nearer the endwall at $y / s=0.36$. The division of the endwall vorticity into two vortices that are weaker than that of the planar case has two significant outcomes. First, the convection of fluid across the passage and up the suction surface has been reduced as the cross-flow between the vortices and the endwall has been weakened. This results in less mixing of high and low loss fluid and decreases the consequent mixing loss. Secondly, the weaker vorticity at the core of two vortices results in less viscous dissipation than that in a single strong vortex. This is evident in the lack of a high loss region typically associated with the core of a vortex in Figure 6.5d.

Figure 6.6 presents the results for the first of the two downstream measurement planes, at $120 \% B_{x}$ downstream of the leading edge of the cascade. The secondary velocity vectors for every second measurement point have been omitted for clarity and the trailing edge projections are shown as dashed lines. The flood of streamwise vorticity coefficient has been paired with line contours of total pressure loss coefficient in order to show the relationship between loss cores and the various vortical structures. Figure $6.6 \mathrm{~b}$ shows a pattern typical of a linear cascade with a planar endwall. The loss core associated with the passage vortex is comprised of fluid of negative vorticity. Near the endwall, below and to the left of the passage vortex is a high loss region of fluid having positive vorticity corresponding to the 
corner vortex. Above and to the left of the passage vortex is fluid of moderate loss having strong positive vorticity. Various authors have described this vortex in different terms and have suggested numerous explanations for its origins. For example, Hodson and Dominy Hodson and Dominy (1987a) and Perdichizzi and Dossena Perdichizzi and Dossena (1993) suggest that it is trailing-edge shed vorticity. Alternatively, the flow visualization results and probe measurements of Benner et al. $(2004 b)$ and those of the previous chapter suggest that it is vorticity generated within the blade passage near the suction surface, by the spanwise velocity induced around the passage vortex. The line contours of $C_{P o}$ show that higher energy fluid is being convected between the passage vortex and this counter rotating vortex. A third region of high loss is generated above and to the left of the passage vortex between about $20 \%$ and $30 \%$ of span. Displaced suction surface boundary layer fluid, and fluid convected around the passage vortex within the blade passage are thought to reside here.

The results for the contoured configuration in Figure $6.6 \mathrm{~d}$ are similar to the planar case except that the negative vorticity is again divided into two regions. The one closest to midspan is thought to correspond to the pressure side leg of the horseshoe vortex that forms around the leading edge of the blade. The vorticity in the core closest to the endwall is believed to develop as the new endwall boundary layer that forms within the blade passage is turned toward the suction side. This is analogous to the development of the secondary flow in a rectangular duct when the boundary layer is subjected to a transverse pressure in the radial direction of the bend. In most high-turning low-pressure turbine blade rows this endwall boundary layer vorticity is fed into the pressure side leg of the horseshoe vortex and the two are indistinguishable, but here the endwall profiling appears to decouple the two. The net result is two weaker vortices that do not directly coincide with peaks of total pressure loss. The strength of either of these vortices is roughly half that of the passage vortex for the planar case, based on the magnitude of streamwise vorticity. There are two 

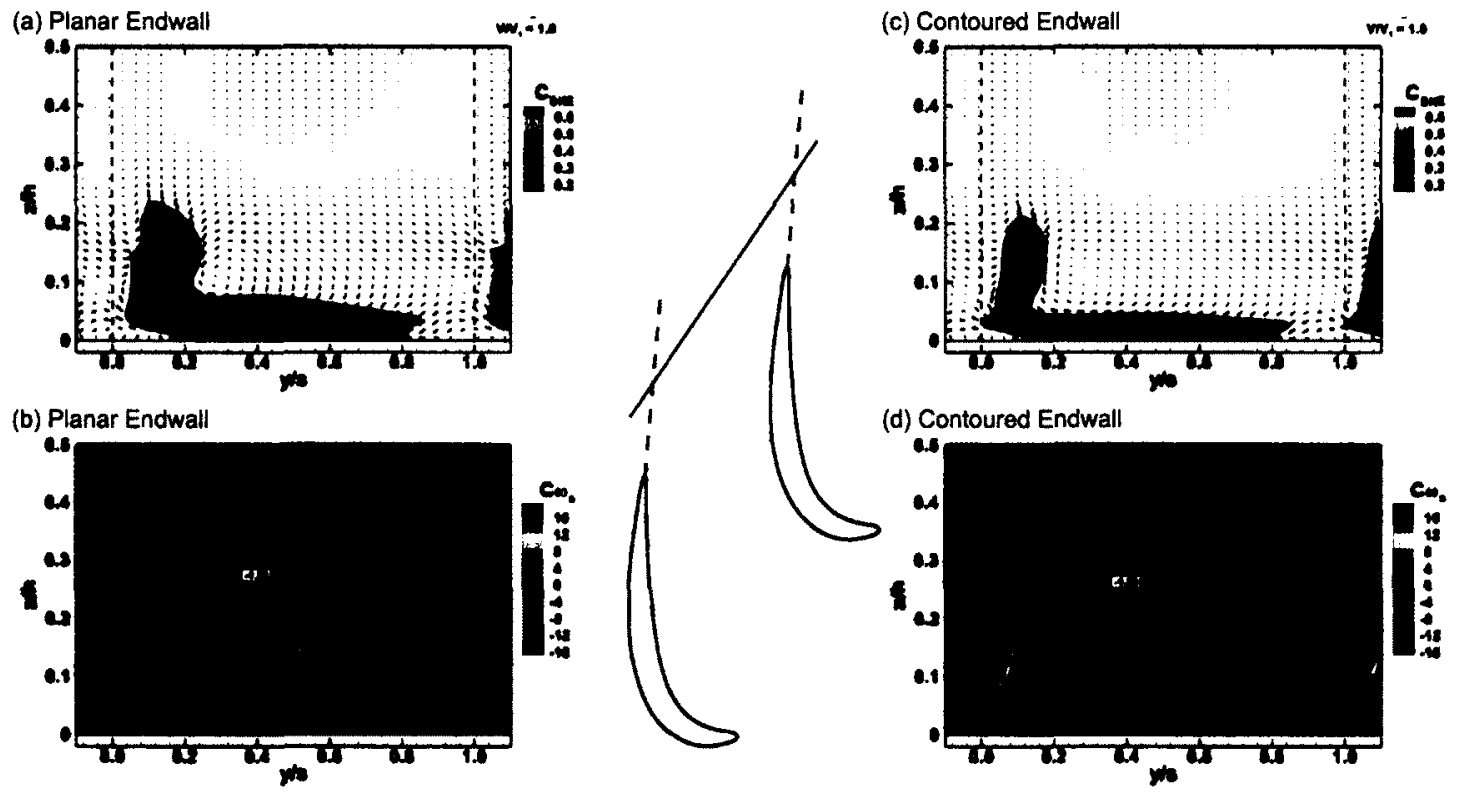

Figure 6.6: Secondary velocity vectors with flood of secondary kinetic energy coefficient $\left(C_{S K E}\right)$ (a \& c), and line contours of total pressure loss coefficient $\left(C_{P o}\right)$ with flood of streamwise vorticity coefficient $\left(C_{\omega s}\right)$ (b \& d), at $120 \%$ axial cord

high loss regions in Figure 6.6d marked by the $C_{P_{o}}=-1.30$ line contours. Both these high loss regions are thought to be comprised of displaced suction surface boundary layer fluid and fluid that is convected across the passage and up the span of the blade. In the case of the high loss region $\left(C_{P o}=-1.30\right)$ closest to the endwall, it is believed that this displaced fluid has been entrained around the pressure-side leg of the horseshoe vortex within the passage.

The floods of secondary kinetic energy coefficient in Figures 6.6a and 6.6c each contain two regions of high magnitude. Close to the endwall, the secondary kinetic energy highlights the pitchwise cross-flow. Away from the endwall, located between the passage vortex and the counter-rotating vortex above and to its left, a second region of high $C_{S K E}$ exists. This secondary kinetic energy is associated to flow that has secondary velocity vectors which point away from the endwall and slightly toward the pressure side of the passage. It appears that 
the degree to which the vectors are turned toward the pressure side, and their magnitude, is linked to the strength of the clockwise rotating structure to their right. This secondary velocity convects fluid between the vortical structures and its effect can be seen in the line contours of total pressure loss coefficient. The strength of cross flow near the endwall is approximately the same for the two test cases, while the spanwise flow is 1.5 times stronger for the planar case.

The second downstream measurement plane was taken $140 \% B_{x}$ from the leading edge of the cascade, and the results are presented in Figure 6.7. Comparing the floods of $C_{S K E}$ to those from $120 \% B_{x}$ reveals an interesting trend: the planar endwall case dissipates more secondary kinetic energy than the contoured one over the same axial distance. At the downstream measurement plane, the planar endwall (Figure 6.7a) continues to have stronger spanwise flow away from the endwall, while the contoured case (Figure 6.7c) has stronger cross-flow near the wall.

Several points regarding the line contours of total pressure loss and the vortical structures are worth addressing. Beginning with the development of the passage vortex for the planar endwall, from Figure $6.6 \mathrm{~b}$ to Figure $6.7 \mathrm{~b}$, the vortex is seen to move away from the endwall and diffuses. In doing so the level of maximum vorticity at the core reduces, which is consistent with the conservation of angular momentum. Also noteworthy is the fact that the peak loss remains constant. In a typical diffusion process one would expect that the peak loss drops as the structure diffuses. Here it appears that the viscous dissipation at the core of the vortex dominates over the diffusion. The loss structure comprised of displaced suction surface boundary layer fluid also diffuses, but its maximum loss is reduced indicating that the diffusion dominates there, over the effect of mixing losses. For the contoured case, both structures having negative vorticity diffuse and weaken, but do not migrate further from the endwall in going from Figure 6.6d to Figure 6.7d. Both regions of high loss fluid diffuse and see their peak loss reduced. 

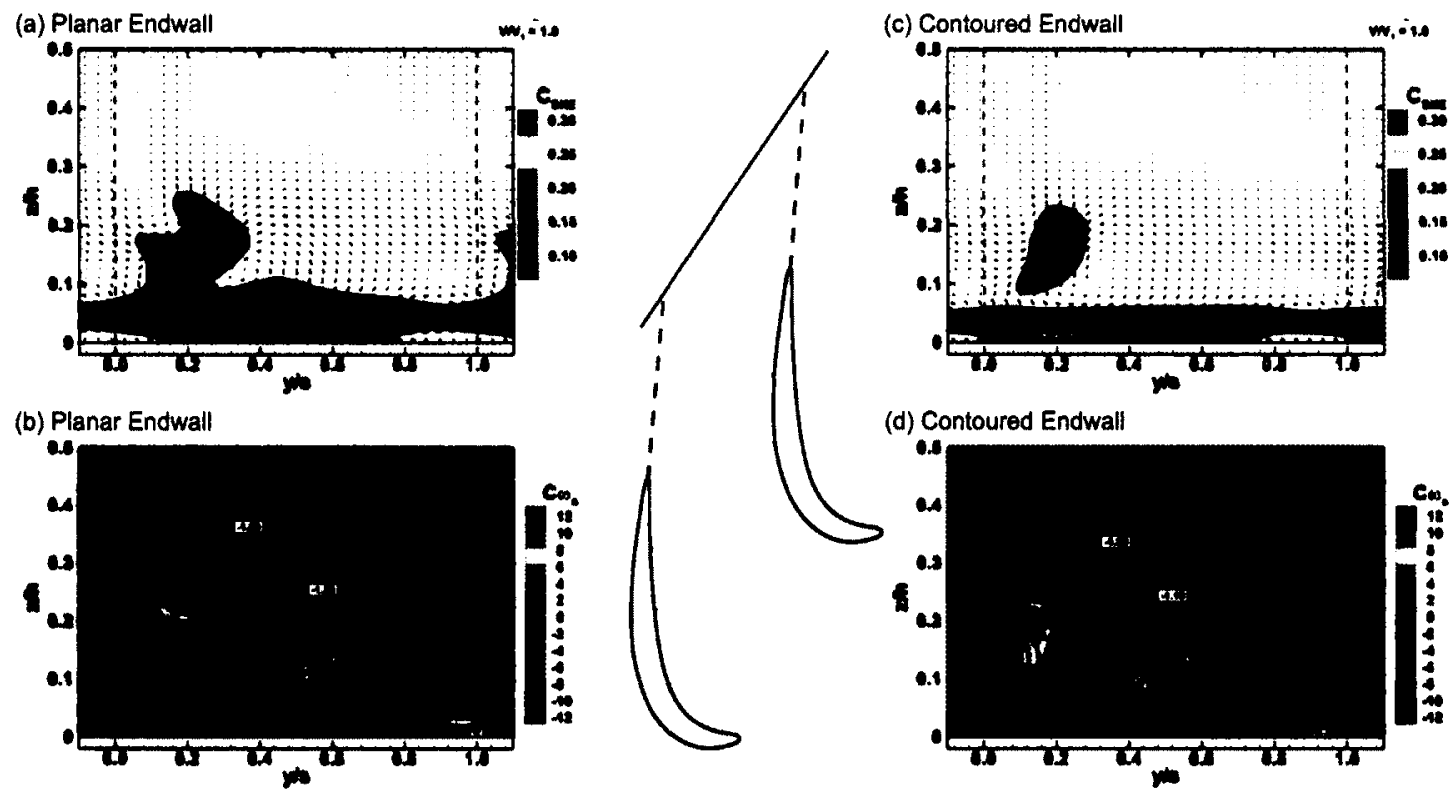

Figure 6.7: Secondary velocity vectors with flood of secondary kinetic energy coefficient $\left(C_{S K E}\right)$ (a \& c), and line contours of total pressure loss coefficient $\left(C_{P o}\right)$ with flood of streamwise vorticity coefficient $\left(C_{\omega s}\right)$ (b \& d), at $140 \%$ axial cord

Comparing the results of the contoured endwall relative to the planar wall at $140 \% B_{x}$ it is apparent that the contouring reduces the overall size of the secondary flow structure and decreases the strength of the various vortices within it.

\subsubsection{Flow Visualization Results}

The oil surface flow visualization results are overlaid with interpreted streamlines for the planar endwall case in Figure 6.8. As before, the nomenclature of Sieverding (1985) is used to identify the streamlines. On the endwall (Figure 6.8a), the flow enters the figure from the right and exits nominally at the top. The saddle point $\left(A_{1}\right)$ marks the location where the incoming stagnation streamline meets the primary separation $\left(S_{1}\right)$ of the inlet boundary layer. The liftoff of the horseshoe vortex that wraps around the leading edge of 
the airfoil is marked $S_{2}$. In Section 5.5.3 it was shown that the pressure side leg of the horseshoe vortex convects across the passage (shown by $S_{2 \mathrm{P}}$ ) and evolves into the passage vortex.

The new boundary layer that forms downstream of $S_{2 \mathrm{P}}$ is swept toward the suction side of the passage and underneath the passage vortex by the pitchwise cross flow near the endwall. Downstream of the arrowheads the surface shear stress is high over most of the endwall as nearly all of the oil and pigment have been scoured away. The suction side leg of the horseshoe vortex $\left(S_{2 \mathrm{~S}}\right)$ encounters the suction surface of the airfoil at approximately $25 \%$ $S / S_{\max }$ and its continuation can be seen in Figure $6.8 \mathrm{~b}$. The last noteworthy separation, $S_{3}$ corresponds to the corner vortex that counter-rotates relative to the passage vortex in the junction of the suction surface with the endwall.

The suction surface flow visualization is presented in Figure $6.8 \mathrm{~b}$. The view is oriented with the leading edge at the top. A ruler noting the fraction of suction surface length is positioned where the blade would meet the endwall. $R_{3}$ is the reattachment of $S_{3}$ which bounds the corner vortex. The penetration of the passage vortex up the span of the blade is shown by $S_{4}$, with the penetration height defined by where it encounters the trailing edge. As was seen on the endwall, $S_{2 S}$ tracks the liftoff of the suction side leg of the horseshoe vortex. Near $S / S_{\max }=0.4, S_{2 \mathrm{~S}}$ takes a peculiar turn in the spanwise direction around a region of low surface shear stress believed to contain separated fluid. Also, the lack of a two dimensional separation at midspan is a notable achievement for an airfoil of this loading level. The lack of separation bubble is in agreement with the loading measurements of Popović (2005).

Figure $6.8 \mathrm{c}$ shows the junction of the suction surface with the endwall. The flow nominally enters from the right and leaves through the upper left. The figure illustrates how the separation streamline corresponding to the suction-side leg of the horseshoe vortex 
(a)

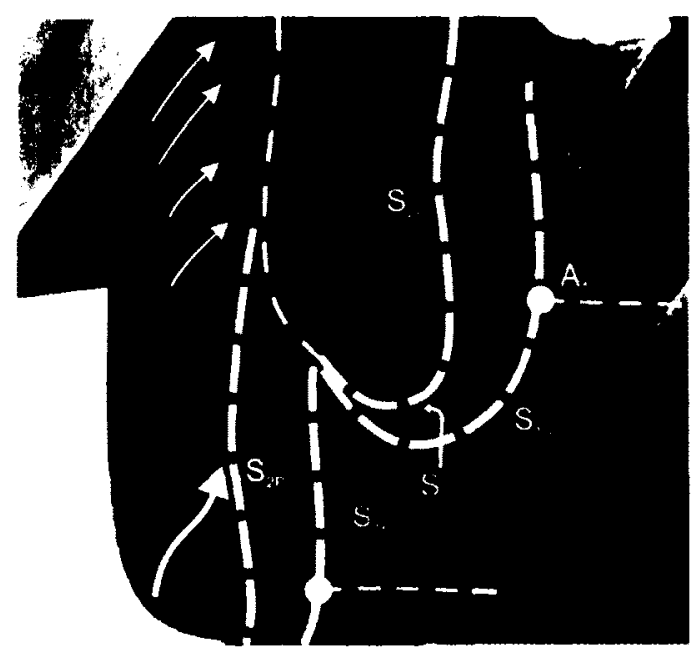

(b)

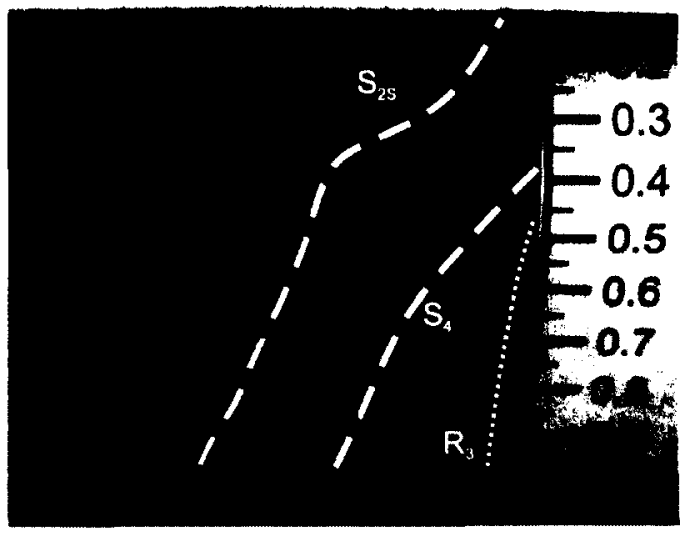

(c)

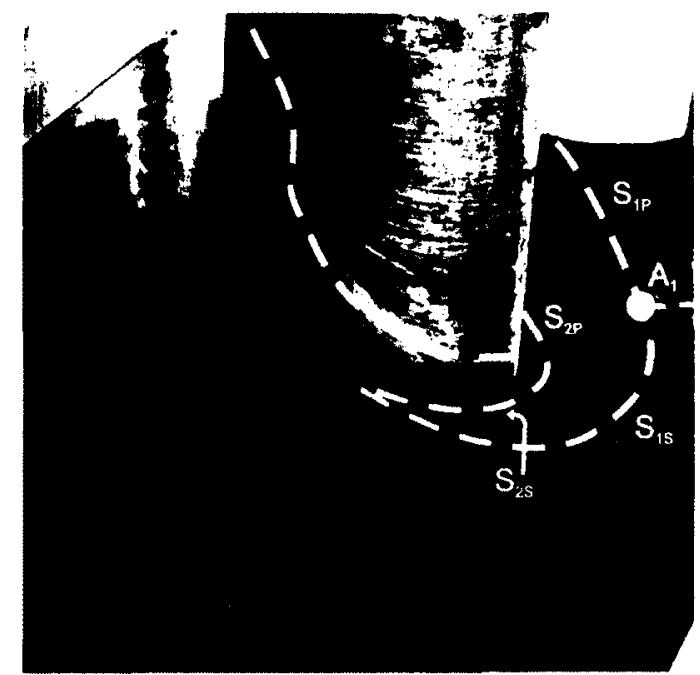

Figure 6.8: Planar endwall oil surface flow visualization showing a) endwall b) blade suction surface c) suction surface - endwall junction 
$\left(S_{2 \mathrm{~S}}\right)$ marks the position of the vortex on both the endwall and the suction surface. The primary separation of the inlet boundary layer $\left(S_{1}\right)$ and the saddle point $\left(A_{1}\right)$ are also marked on the endwall.

Figure 6.9 presents oil surface flow visualizations for the contoured test case. The approximate locations of the hill $(H)$ and valleys $\left(V_{1}\right.$ and $\left.V_{2}\right)$ have been marked. The streamline curvature that results from the presence of a valley causes a relative diffusion of the flow, raising the local static pressure (Rose, 1994). This increase in static pressure can be seen in Figure 6.9a where the pressure side legs of $S_{1}$ and $S_{2}$ are displaced upstream compared to their location in the planar endwall case. The new boundary layer that forms downstream of $S_{2 \mathrm{P}}$ is divided into two parts. The fluid between $S_{2 \mathrm{P}}$ and the long arrow is rolled up into the passage vortex. The boundary layer forming downstream of the long arrow rolls up to form the core of negative vorticity closest to the endwall seen in Figure $6.6 \mathrm{~d}$.

The suction surface flow visualization for the contoured test case is shown in Figure 6.9b. The blade is recessed into the endwall and the height of the contouring is shown as the solid line. The penetration height of the secondary flow (the location where $S_{4}$ meets the trailing edge) has been reduced by approximately $1 \mathrm{~cm}$ compared to the planar endwall case. This is consistent with the measured reduction in secondary losses. The increased endwall static pressure resulting from the presence of valley $V_{1}$ causes $S_{2 \mathrm{P}}$ to shift toward midspan near it's origin at $S / S_{\max }=0.25$.

\subsubsection{Summary of Results}

The exit plane measurements at $140 \% B_{x}$ are analyzed by pitchwise mass-averaging in Fig 6.10. The total pressure loss coefficient in Figure 6.10a has been normalized by the outlet midspan dynamic pressure. From the endwall to approximately $8 \%$ span the 
(a)

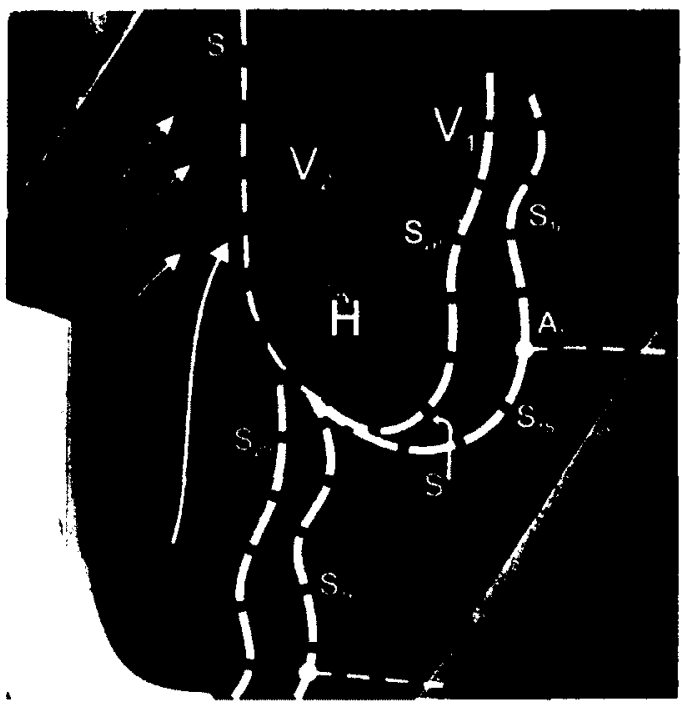

(b)

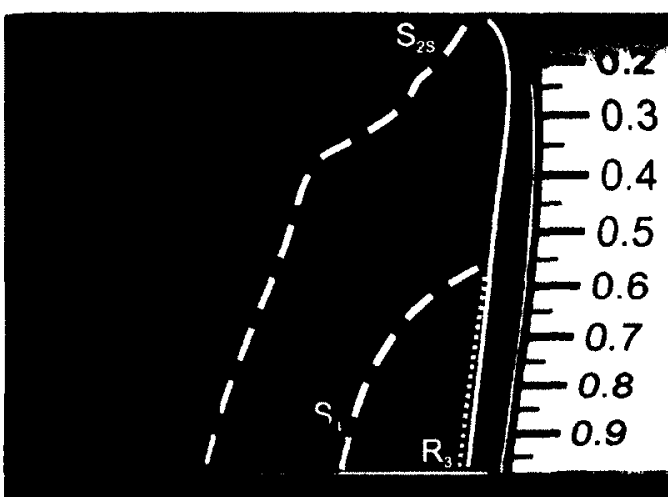

Figure 6.9: Contoured endwall oil surface flow visualization showing a) endwall b) blade suction surface

contoured endwall produces higher loss than the planar, possibly a result of the reduced convection of fluid away from the endwall by the spanwise flow discussed above. The curve for the planar endwall has two defined loss peaks. The loss at $11 \%$ span coincides with the core of the passage vortex. The contoured case also produces high losses focused at two spanwise locations. The loss at $z / h=0.06$ roughly coincides with the core of the negative vorticity closest to the endwall. The mass-averaged and mixed-out mass-averaged exit flow results are summarized in Table 6.1. The endwall contouring reduces the mixed-out $Y_{\text {total }}$ by $18 \%$ and mixed-out $Y_{\text {secondary }}$ was reduced by $32 \%$. 
The benefit measured here is roughly double what was found in the previous chapter for the application of endwall contouring to PAKB. Comparing the results from the present data set with those of Section 5.5.4, it can be inferred that front-loaded high-lift airfoils are prime candidates for endwall contouring. The strong pitchwise pressure gradient in the early part of the passage develops a strong secondary flow which can be mitigated with endwall profiling.

The airfoil used in the current study, PAKD-F, is a high-lift, front-loaded derivative of PAKB with very good stall characteristics. With the application of endwall contouring, the secondary loss of PAKD-F is brought within $25 \%$ of the baseline airfoil. This implies that the $25 \%$ increase in loading between the two blades is perhaps ambitious; A front-loaded airfoil with more moderate loading could give equivalent loss behaviour to PAKB over a wider range of operating conditions, while achieving a weight reduction. This concept is discussed in Section 8.2.4. It is also conceivable that the amplitude of the contouring could be increased in an attempt to further reduce the secondary losses of PAKD-F. However, increasing displacement amplitudes excessively can lead to the separation of the endwall boundary layer and higher losses, as was found by Ingram et al. (2005) for their aggressive profile design.

Figure $6.10 \mathrm{~b}$ shows that both configurations produce an over/underturning of the flow typical of a turbine blade row. The extent of the over/underturning has been linked to the strength of the passage vortex and the amount of secondary loss generated. The contouring reduces the maximum peak underturning of the flow by 2.8 degrees. The measured massaveraged flow turning of the blade row $\left(\alpha_{2}^{\prime \prime}\right)$ is documented in Table 6.1 and was increased by a negligible amount with the application of contouring. Holley (2004) notes that the mass-averaged exit yaw angle only partially represents the tangential force on the blade, and that the representation becomes less accurate as the exit yaw angle is decoupled from the axial velocity. An endwall contour that reduces loss but at the same time unloads the 
blade at the root would be undesirable. The measured tangential force on the blade $\left(F_{Y}\right)$ has been computed from the change in momentum between the inlet measurement plane and the $140 \% B_{x}$ plane, and the results are listed in Table 6.1. $F_{Y}$ has not changed with the contouring to within the error of the experiment. Thus the reduction in loss is not associated with unloading the blade. It should be noted that the force exerted on the flow by the profiled endwall is neglected in the analysis, though it is expected to be small based on the computational results.
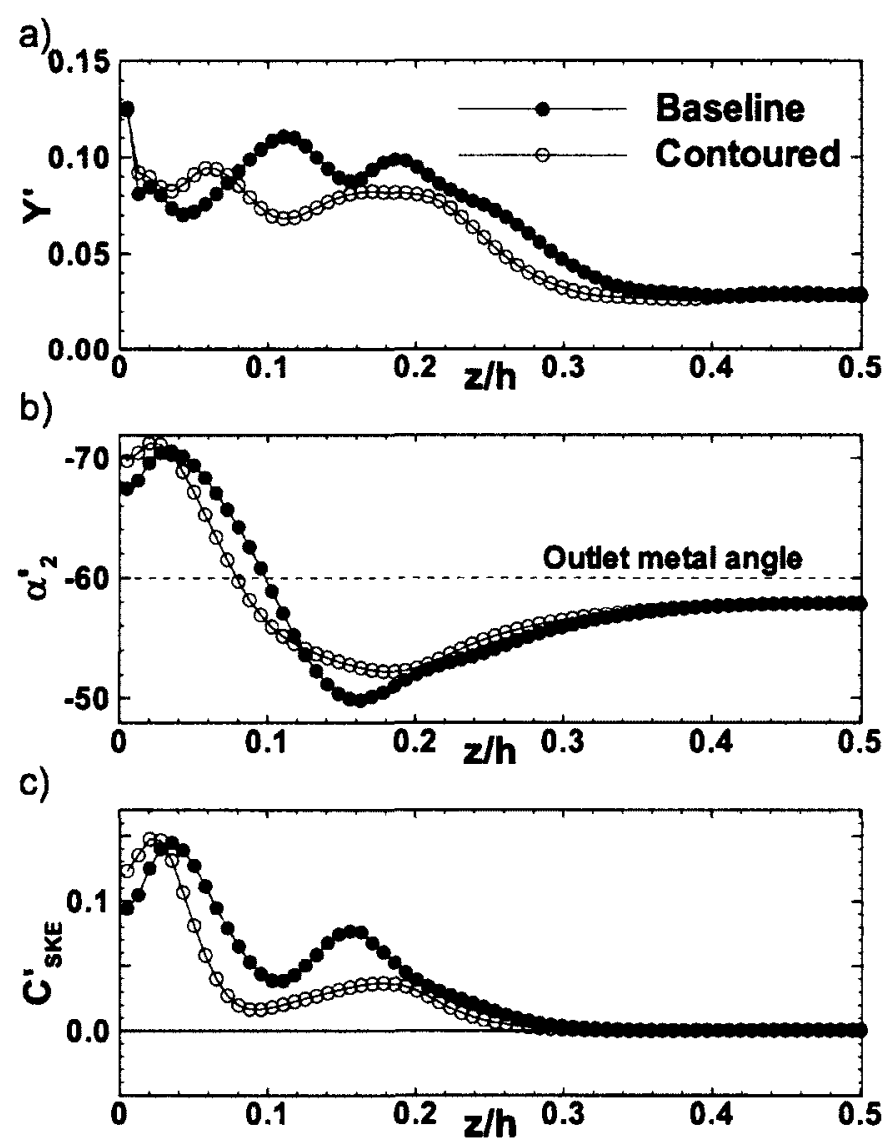

Figure 6.10: Pitchwise mass-averaged results for the $140 \% B_{x}$ measurement plane (a) total pressure loss coefficient, (b) outlet flow angle and (c) secondary kinetic energy coefficient

The pitchwise mass-averaged coefficient of secondary kinetic energy is presented in Figure $6.10 \mathrm{c}$. Contouring the endwall serves to reduce the $C_{S K E}$ everywhere but the first 
$3 \%$ of span from the endwall where it is a direct consequence of increased overturning. Overall the mass-averaged secondary kinetic energy is reduced by $35 \%$ by the presence of the contouring. This reduction of $C_{S K E}$ has a significant impact on the mixing loss incurred between the measurement plane and the hypothetical mixed-out plane. A breakdown of the mixing loss is summarized in Table 6.2 for the $120 \%$ and $140 \% B_{x}$ measurement planes.

Table 6.2: Loss breakdown - mixing losses from measurement plane

\begin{tabular}{lcccc}
\hline \hline & \multicolumn{2}{c}{ PLANAR } & \multicolumn{2}{c}{ CONTOURED } \\
QUANTITY & $120 \% B_{x}$ & $140 \% B_{x}$ & $120 \% B_{x}$ & $140 \% B_{x}$ \\
\hline$\Delta$ Primary KE & -0.075 & -0.053 & -0.061 & -0.045 \\
$C_{P}$ Recovery & 0.063 & 0.046 & 0.051 & 0.038 \\
Diffusion Loss & -0.012 & -0.007 & -0.010 & -0.007 \\
$\Delta$ Secondary KE & -0.039 & -0.031 & -0.023 & -0.020 \\
Mixing Loss & -0.051 & -0.039 & -0.033 & -0.027 \\
\hline Measured Loss & -0.132 & -0.150 & -0.114 & -0.127 \\
Mixed-Out Loss & -0.183 & -0.188 & -0.148 & -0.154 \\
\hline \hline
\end{tabular}

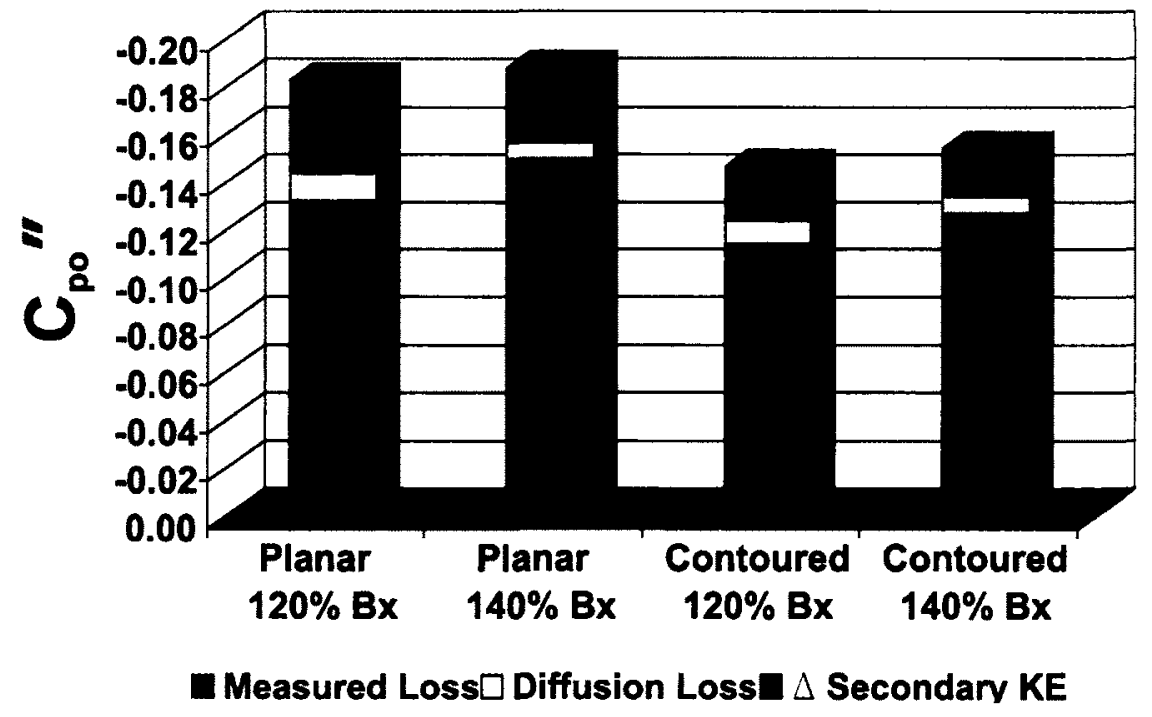

Figure 6.11: Contributions to mixed-out loss

The mass-averaged quantities (normalized by inlet midspan dynamic pressure) listed in Table 6.2 are shown graphically in Figure 6.11. The diffusion loss is defined as the change in primary kinetic energy between the measurement plane and mixed-out plane that is not recovered as static pressure. The mixing loss is the sum of the diffusion loss and the change 
in secondary kinetic energy. At the downstream measurement planes, both the planar and contoured endwalls produce the same diffusion loss, but the planar case generates $44 \%$ higher mixing loss strictly resulting from higher secondary kinetic energy. Therefore contouring the endwall serves not only to reduce the loss at the measurement plane, but the mixing loss as well.

The diffusion loss for both planar and contoured configurations is higher at $120 \% B_{x}$ relative to $140 \% B_{x}$. This is expected as the primary velocity field has larger non-uniformity closer to the trailing edge of the cascade. The secondary kinetic energy is also higher for both test cases at the upstream plane, however, the measured loss is lower. The net result is that the mixed-out loss for the $120 \%$ plane is slightly less than that found at the exit plane. Moore and Adhye (1985) found that the contribution of wall shear stress to loss was small downstream of $140 \% B_{x}$, thus, the increase in mixed-out loss could be attributed to viscous dissipation in the endwall boundary layer.

\subsection{Conclusions}

As was found by previous researchers, this chapter confirms that non-axisymmetric endwall contouring can be used to mitigate endwall loss. However, this chapter investigates the application of endwall contouring to a linear cascade that is much more highly loaded than has previously been published. The body of experimental data shows that substantial loss reductions are possible when endwall profiling is applied to a high-lift front-loaded airfoil. Past research conducted at Carleton University has found that front-loading a highlift design is attractive from the perspective of midspan performance, avoiding the burden of a separation bubble and offering good stall characteristics over a wide range of operating conditions. The current chapter shows that the application of endwall contouring to this airfoil reduces the secondary losses and yields appealing overall blade row performance. In 
conjunction with the previous work, this chapter demonstrates the potential feasibility of high-lift airfoils.

The discussion focuses on the physics of the flow within the blade passage as well as downstream of the trailing edge, and the principal loss reduction mechanisms are examined. The measurements show that the contoured endwall divides the vorticity typically associated with the passage vortex into two vortices. One of these vortices is attributed to the pressure side leg of the horseshoe vortex that normally evolves into the passage vortex. The other vortex is thought to develop as the new boundary layer that forms between the blades is turned by a transverse pressure gradient. The benefit of two weaker vortices over one stronger vortex is three-fold. First, the weaker vortices convect less higher energy fluid from the pressure side of the passage into the secondary flow structure, reducing the amount of mixing. Second, the vorticity at the core of the vortices is lower, resulting in less viscous dissipation. Third, the measured secondary kinetic energy is reduced thereby lessening the subsequent mixing loss. These three mechanisms result in a smaller secondary loss structure with lower peak loss.

Finally, this chapter builds on the ground work laid by the previous one. This work marks an intermediate stage in the research program investigating secondary loss reduction for high-lift low-pressure turbine airfoils. The following chapters will examine the effect of overall loading level and loading distribution on loss generating mechanisms through experimental and computational avenues. 


\section{Chapter 7}

\section{The Effect of Loading Distribution}

\section{on Blade Row Loss in}

\section{Highly-Loaded Linear Cascades}

\section{with Planar Endwalls}

\subsection{Introduction}

The previous chapters have demonstrated the effectiveness of non-axisymmetric endwall contouring for reducing the size and strength of endwall flows. They have also shown it to be useful for increasing blade loading without suffering inherently higher secondary losses. The present chapter investigates a different, novel approach to secondary loss reduction. The secondary loss reduction is achieved by modification of the two-dimensional profile of the linear cascade blade. 


\subsection{Motivation}

Like the previous chapter, the goal here is to demonstrate the potential use of more highly-loaded low-pressure turbine blades for engine weight reduction. The previous chapter showed that a forward-loaded airfoil, having a loading level $25 \%$ higher than that of the baseline test case can be used in conjunction with non-axisymmetric endwall contouring with limited penalty in blade row performance. Rather than a three-dimensional modification of the endwall, this chapter considers altering the two-dimensional cross-section of the blade. The basis for the modification in loading arises from the notion that the lower the streamwise momentum of the fluid, the more strongly it will be driven across the blade passage by the pressure gradient in that direction. Traditionally, front-loaded airfoils have a large region of near-stagnation fluid on the forward pressure side of the airfoil. The resulting low momentum fluid near the endwall is driven across the passage and rolls up into the passage vortex forming a strong secondary flow. This high pressure region can be alleviated by increasing the streamwise momentum of the flow and thus reducing the static pressure. The pressure on the suction-side of the passage must then also be reduced to maintain the original loading level. This modified-loading method has the advantage that it does not require the added mechanical complexity and manufacturing cost of a contoured endwall.

Conventionally, blade loading distribution has been examined in the context of aft versus forward loaded; that is, whether the suction peak occurs forward or aft of mid chord. Patterson and Hoeger (1986) investigated the boundary layers on the suction surface of three low-pressure turbine cascades having varying loading distributions. They found that aft-loading delayed transition to turbulence and generated lower profile loss. Hoheisel et al. (1987) describe the design of the T104, T105 and T106 airfoils having approximately the

same loading level. The front-loaded T104 was designed with the intent of obtaining a pressure distribution that avoids a strong decelerating region. This is achieved with a strong 
acceleration in the forward region of the suction side, followed by a gradual deceleration of the flow toward the trailing edge. The aft loaded T105 and T106 airfoils were designed with the goal of maintaining a laminar boundary layer along the suction surface as long as possible. This is accomplished with a gradual acceleration of the flow along the suction surface to the suction peak. The loading distribution of the T105 and T106 are similar, with the T106 having a somewhat lower peak velocity and subsequently a more gradual deceleration toward the trailing edge. Weiss and Fottner (1995) studied the blade row performance of the T104 and T106, which have a Zweifel coefficient of $Z_{w}=1.07$ and $Z_{w}$ $=1.04$, respectively. They found that the front-loaded T104 generated higher secondary losses and stronger secondary kinetic energy than the aft-loaded T106. Examining the loading distributions of the two airfoils (Hoheisel et al., 1987, Figure 2) reveals that the minimum velocity on the pressure surface of the T104 is approximately $35 \%$ lower than that of the T106. Thus, their findings are consistent with the streamwise momentum argument presented above.

More closely related to the present study, the earlier PAK airfoils (PAKB, PAKD-A and PAKD-F) were examined by Popović (2005) and Zorić (2006), who focused their efforts on mid-span and secondary losses respectively. The baseline cascade, PAKB, has a Zweifel coefficient of $Z_{w}=1.08$. The PAKD airfoils have a loading level $25 \%$ higher than that of the baseline case $\left(Z_{w}=1.36\right)$. The authors found that at the higher blade loading, the aft-loaded PAKD-A had a large separation bubble that dominated the loss generation in the twodimensional midspan boundary layer. Popović (2005) rationalized that the loss generated in the separation bubble superseded the benefit of a longer laminar region. In addition, the separation bubble had the propensity to avoid reattachment at low Reynolds number and positive incidence resulting in blade stall. The front-loaded PAKD-F avoids a separation bubble, except at very low Reynolds number, with a more gradual adverse pressure gradient along the suction surface. As a result, it had better mid-span performance than the aft- 
loaded kin at off-design incidence and Reynolds number. In general, the profile losses of front-loaded blade were lower; however, the secondary flows were significantly stronger and overall the blade row loss was higher. Hence the front-loaded blade is a suitable candidate for the modified-loading secondary loss reduction strategy.

\subsection{Approach}

This chapter investigates the flow field of four linear low-pressure turbine cascades with planar endwalls experimentally and numerically. The first three cascades are the same as those tested by Popović (2005) and Zorić (2006) (PAKB, PAKD-A and PAKD-F). The fourth was designed by Pratt and Whitney Aircraft specifically for the purpose of this research. The new blade pack is forward-loaded, employs the modified-loading method described above and carries the designation PAKD-FP.

The cascades are shown in Figure 3.4 and their geometric details are given in Table 3.2. The work was conducted at design incidence and at a Reynolds number of 126,000 based on inlet velocity and axial chord.

Seven-hole pressure probe measurements were taken on axial planes within the blade passage, at the trailing edge and downstream of the cascades. The flow physics are discussed and the measurements are compared to numerical results. Oil surface flow visualizations were performed on the endwall and blade suction surface and are compared to predicted surface shear stress trajectories. 


\subsection{Results and Discussion}

\subsubsection{Velocity Distributions}

The strength of secondary flows in a turbine cascade are correlated to the overall loading level (Marchal and Sieverding, 1977; Hodson and Dominy, 1987b; Perdichizzi and Dossena, 1993; Zorić, 2006) and loading distribution (Weiss and Fottner, 1995; Zorić, 2006). It is accepted that the static pressure distribution drives the roll up of the passage vortex that forms the bulk of the secondary flow. In this chapter, the influence of the velocity distribution along the pressure surface and its effect on the roll up of the passage vortex is investigated. The mid-span static pressure distributions of the four cascades are presented in Figure 7.1 in the form of a velocity distribution or velocity ratio as defined by:

$$
\frac{V_{\text {local }}}{V_{1}}=\frac{\sqrt{P_{o, 1}-P_{\text {local }}}}{\sqrt{P_{o, 1}-P_{1}}}
$$

The flow stagnates on the pressure surface of the airfoil. Downstream of the stagnation point, the flow accelerates along the pressure surface in the direction of the trailing edge. The distribution downstream of this acceleration is of interest as it appears to drive the roll up of the passage vortex. The region of interest has been highlighted by a dashed ellipse in Figure 7.1. In Section 7.4.2.2 it will be shown that the secondary losses for the cascades vary inversely with the local minimum velocity downstream of the acceleration from the stagnation point along the pressure surface. For PAKD-FP, the flow stagnates along the pressure surface at approximately $5 \%$ axial chord. At $10 \% B_{x}$ the flow begins to decelerate toward a local minimum at about $17 \% B_{x}$ where $V_{\text {local }} / V_{1}=0.41$. PAKD-FP and PAKB have the same minimum value of $V_{l o c a l} / V_{1}$ downstream of the acceleration from the stagnation point; however the local minimum velocity for PAKB occurs at $40 \% B_{x}$. PAKD-A and PAKD-F have minimum values of $V_{\text {local }} / V_{1}$ downstream of acceleration from 


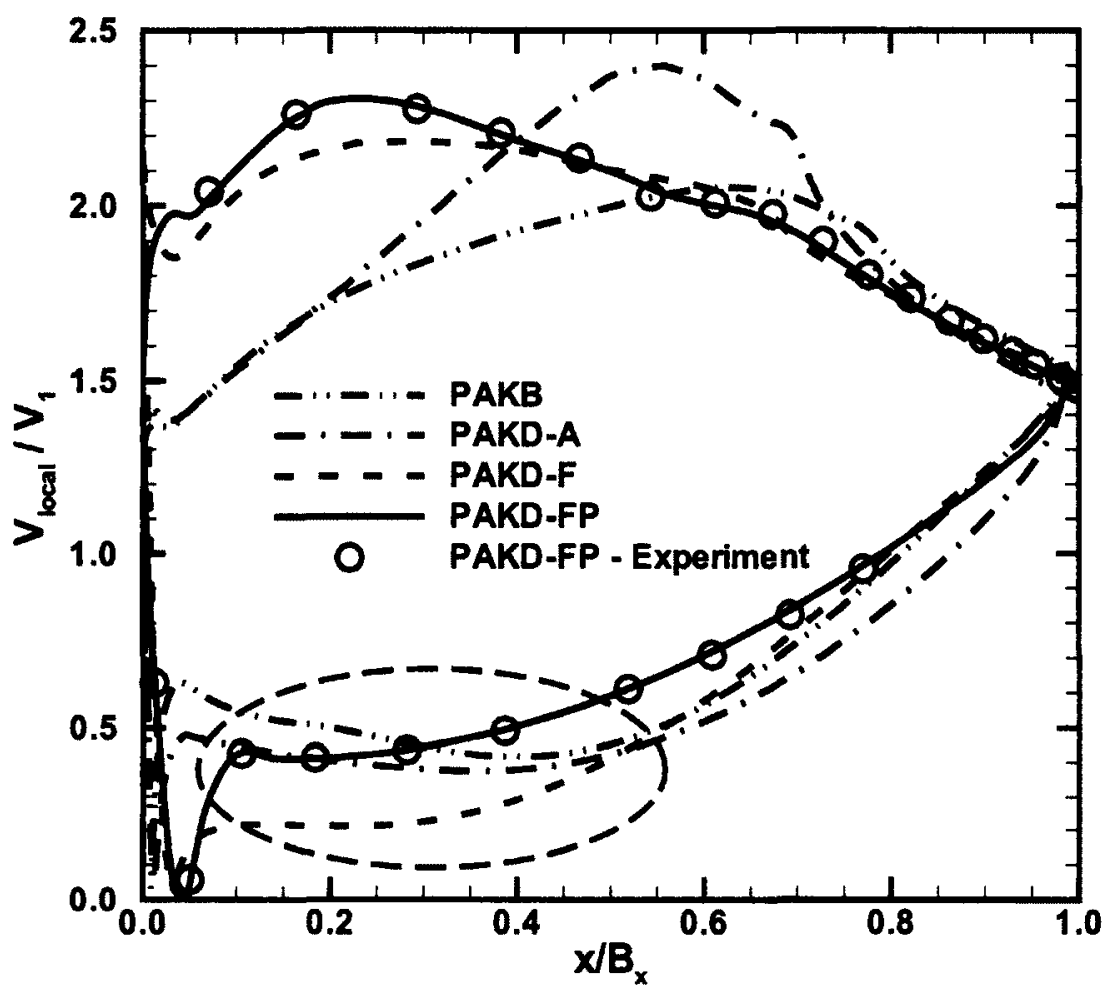

Figure 7.1: Predicted velocity distributions for PAKB, PAKD-A, PAKD-F and PAKD-FP

the stagnation point of 0.38 at $36 \% B_{x}$ and 0.21 at $22 \% B_{x}$, respectively.

Along the suction surface the peak velocity for PAKD-FP occurs $0.05 x / B_{x}$ upstream that of PAKD-F, with the maximum velocity being about $5 \%$ higher. Downstream of the location of maximum velocity the flow decelerates in the direction of the trailing edge. The rate of deceleration downstream of the peak is somewhat greater for PAKD-FP than for PAKD-F albeit considerably less than for PAKD-A. This is noteworthy, as it will be shown in Section 7.4.3 that PAKD-FP has a small suction surface separation bubble while PAKD-F does not. Previous work has shown that the existence and location of a separation bubble is dependent on the pressure gradient history of the boundary layer (Hatman and 
Wang, 1999; Yaras, 2001; Houtermans et al., 2004).

\subsubsection{Measured Flow Field}

The results for the inlet plane, the exit plane $\left(140 \% B_{x}\right)$ and mixed-out results for the four cascades are tabulated in Table 7.1. The following subsections examine the midspan and secondary flow characteristics of the blade packs with particular focus placed on PAKDFP.

\subsubsection{Midspan Flow}

The profile loss for PAKD-FP shows an unexpected trend. The measured $C_{P o_{\text {profile }}}^{\prime}$ is somewhat lower than that of both PAKD-A and PAKD-F (by an amount that is roughly twice the experimental uncertainty). However, the mixed-out profile losses for the three PAKD airfoils are within the experimental error of one another. The two-dimensional wakes that form downstream of blade depend on the boundary layer development on the suction and pressure surfaces, as well as the trailing edge thickness. Since the PAKD airfoils have the same ratio of trailing edge thickness to throat opening, they should generate very similar trailing edge losses (Kacker and Okapuu, 1982). Thus, differences in profile loss result primarily from differences in boundary layer development.

The midspan wakes of the PAKD airfoils, as measured on the exit plane, are presented in Figure 7.2 in the form of the total pressure deficit coefficient $\left(C_{P_{o}}\right)$. The wake of PAKD-FP is narrower and deeper than those of PAKD-F and PAKD-A.

For two dimensional boundary layers Denton (1993) demonstrates that the entropy generation scales on the cube of the boundary layer edge velocity and also the dissipation coefficient to the first power. He also notes that the suction surface boundary layer generates 
Table 7.1: Summary of experimental planar endwall exit plane results for PAKB, PAKD-A, PAKD-F and PAKD-FP

\begin{tabular}{|c|c|c|c|c|c|}
\hline LOCATION & FLOW QUANTITY & PAKB & PAKD-A & PAKD-F & PAKD-FP \\
\hline \multirow[t]{5}{*}{ INLET } & $\delta / h$ & 0.16 & 0.16 & 0.16 & 0.16 \\
\hline & $\delta^{*}[\mathrm{~mm}]$ & 1.9 & 2.0 & 2.2 & 2.1 \\
\hline & $\theta[\mathrm{mm}]$ & 1.6 & 1.9 & 1.8 & 1.8 \\
\hline & $H$ & 1.2 & 1.1 & 1.2 & 1.2 \\
\hline & $C_{P o, 1}^{\prime \prime}$ & 0.00025 & -0.0142 & -0.0027 & -0.0112 \\
\hline EXIT & $C_{P O_{\text {tota }}}^{\prime \prime}$ & -0.098 & -0.126 & -0.150 & -0.112 \\
\hline \multirow[t]{12}{*}{$B_{x}=1.40$} & $C_{P o}^{\prime}$ & -0.057 & -0.069 & -0.067 & -0.062 \\
\hline & $C_{P_{\text {secondary }}}^{\prime \prime}$ & -0.041 & -0.057 & -0.083 & -0.051 \\
\hline & $Y_{\text {total }}$ & 0.043 & 0.058 & 0.063 & 0.049 \\
\hline & $Y_{\text {profile }}$ & 0.024 & 0.031 & 0.028 & 0.027 \\
\hline & $Y_{\text {secondary }}$ & 0.019 & 0.027 & 0.035 & 0.022 \\
\hline & $C_{S K E}^{\prime \prime}$ & 0.015 & 0.021 & 0.031 & 0.017 \\
\hline & $\alpha_{2}^{\prime \prime}[\mathrm{deg}]$ & -57.2 & -56.1 & -56.8 & -56.9 \\
\hline & $\alpha_{2, \text { midspan }}^{\prime}[\mathrm{deg}]$ & -57.7 & -57.6 & -57.9 & -57.9 \\
\hline & $q_{2}^{\prime \prime} / q_{C L_{1}}$ & 2.334 & 2.195 & 2.334 & 2.323 \\
\hline & $q_{2, \text { midspan }}^{\prime} / q_{C L_{1}}$ & 2.312 & 2.224 & 2.359 & 2.296 \\
\hline & $A V R$ & 0.979 & 0.976 & 0.996 & 0.959 \\
\hline & $F_{Y}$ & 3.50 & 4.23 & 4.41 & 4.40 \\
\hline \multirow[t]{10}{*}{ MIXED-OUT } & $C_{P O_{\text {to }}}^{\prime \prime}$ & -0.117 & -0.154 & -0.188 & -0.134 \\
\hline & $C_{P o}^{\prime}$ & -0.058 & -0.072 & -0.075 & -0.073 \\
\hline & $C_{P o_{\text {secondary }}}^{\prime \prime}$ & -0.059 & -0.082 & -0.113 & -0.062 \\
\hline & $Y_{\text {total }}$ & 0.052 & 0.072 & 0.082 & 0.059 \\
\hline & $Y_{\text {profile }}$ & 0.025 & 0.032 & 0.032 & 0.031 \\
\hline & $Y_{\text {secondary }}$ & 0.027 & 0.040 & 0.050 & 0.028 \\
\hline & $\alpha_{2}^{\prime \prime}[\mathrm{deg}]$ & -57.7 & -56.8 & -57.7 & -57.5 \\
\hline & $\alpha_{2, \text { midspan }}^{\prime}[\mathrm{deg}]$ & -57.7 & -57.7 & -58.0 & -57.9 \\
\hline & $q_{2}^{\prime \prime} / q_{C L_{1}}$ & 2.259 & 2.219 & 2.259 & 2.268 \\
\hline & $q_{2, \text { midspan }}^{\prime} / q_{C L_{1}}$ & 2.309 & 2.130 & 2.352 & 2.323 \\
\hline
\end{tabular}

the bulk of the profile loss. The dissipation in a boundary layer can be interpreted as the viscous shear work being converted to heat. It is dependent on the state of the boundary layer (laminar, turbulent etc.) and the pressure gradient history seen from the stagnation point to the trailing edge. In general, a boundary layer subjected to a favourable streamwise pressure gradient (accelerating) is thinner, but has higher shear gradients in the wall normal direction than one having zero streamwise pressure gradient. As a result of this 


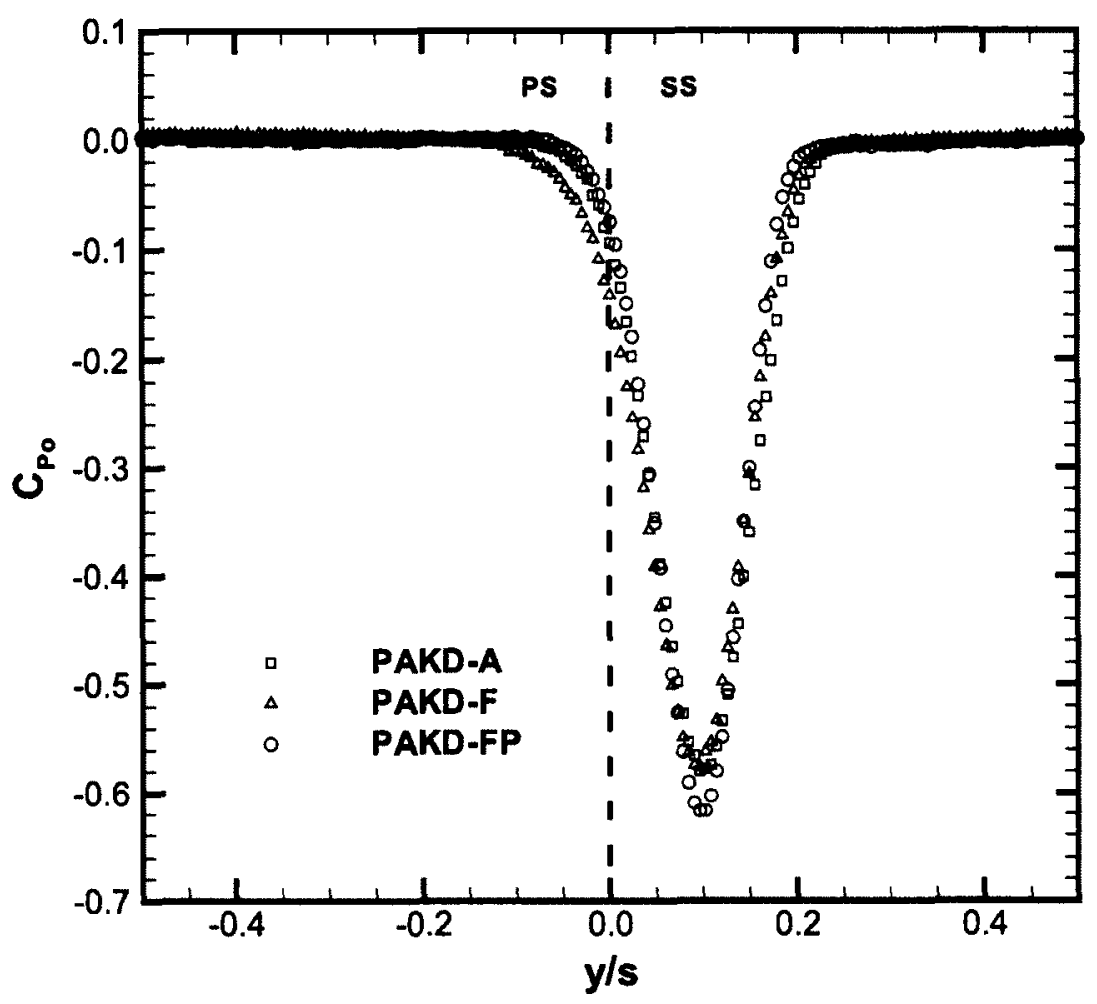

Figure 7.2: Total pressure deficit coefficient for the midspan wakes of PAKD-A, PAKD-F and PAKD-FP as measured at the exit plane

an accelerating boundary layer generates less dissipation and produces a thinner, deeper wake. For the case of PAKD-FP, it appears that the strong acceleration in the forward part of the passage dominates the other loss generation mechanisms as it produces a thinner wake than those of PAKD-F and PAKD-A, having lower mass-averaged measured loss at the exit plane.

However, the narrow, deep wake has to undergo more diffusion in order to reach the mixed-out condition. In a two-dimensional wake, the mixing loss is a result of the change in kinetic energy between the measurement and mixed-out planes that is not recovered as static pressure rise. Since the wakes of PAKD-F and PAKD-A are more diffuse and closer to 
the mixed out condition at the measurement plane, they generate less mixing loss. The end result is that the three cascades produce roughly the same final mixed-out profile loss. It is noteworthy that the PAKD cascades generate $28 \%$ higher mixed-out midspan loss ( $Y_{\text {profile }}$ ) than the baseline test case PAKB, as a result of their $25 \%$ higher aerodynamic loading.

\subsubsection{Endwall Flow}

The endwall flow is summarized in Table 7.2. The minimum $V_{\text {local }} / V_{1}$ is the lowest value of pressure-surface velocity ratio occurring locally downstream of the acceleration from the stagnation point taken from Figure 7.1. The table is sorted in order of increasing mixed-out secondary loss $\left(Y_{\text {secondary }}\right)$, which varies inversely with the minimum pressuresurface velocity ratio. The table also shows that the secondary loss is proportional to the measured secondary kinetic energy. The results for PAKD-FP are nearly identical to those of the baseline test case PAKB. This is remarkable as PAKD-FP has $25 \%$ higher loading than PAKB and the secondary loss and secondary kinetic energy are commonly accepted to be proportional to loading. It is also worth mentioning that PAKD-F has a minimum $V_{\text {local }} / V_{1}$ half that of PAKD-FP and generates nearly double the mixed-out secondary loss and secondary kinetic energy.

Table 7.2: Summary of minimum pressure-surface velocity ratio, mixed-out secondary loss and secondary kinetic energy

\begin{tabular}{llll}
\hline \hline Airfoil & $\begin{array}{l}\text { Minimum } \\
V_{\text {local }} / V_{1}\end{array}$ & $\begin{array}{l}\text { Mixed-out } \\
Y_{\text {secondary }}\end{array}$ & $\begin{array}{l}\text { Measured } \\
C_{S K E}^{\prime \prime}\end{array}$ \\
\hline PAKB & 0.42 & 0.027 & 0.015 \\
PAKD-FP & 0.42 & 0.028 & 0.017 \\
PAKD-A & 0.38 & 0.040 & 0.021 \\
PAKD-F & 0.21 & 0.050 & 0.031 \\
\hline \hline
\end{tabular}

The intra-passage measurements for the four cascades are examined next with particular attention to the flow physics. Figures 7.3 (a, b, c \& d) show colour flood contour plots of secondary kinetic energy coefficient $\left(C_{S K E}\right)$, overlaid with secondary velocity vectors. The 
$C_{S K E}$ scales as the square of the secondary velocity so it amplifies subtle differences in the magnitude of the secondary velocity vectors. As was discussed in the previous chapters, the secondary kinetic energy in the blade passage is localized into two regions. The first region near the endwall is associated with the pitchwise cross-flow. The second region is related to flow away from the endwall along the suction surface of the blade. The results of the previous two chapters suggested that the cross-flow into the suction-side-endwall junction was driven by the strength of the cross-passage pressure gradient. Also, the last chapter implied that forward-loading generated a stronger cross-passage flow. However, Figures 7.3 $(a, b, c \& d)$ indicate that the streamwise momentum of the fluid has a significant influence as well since PAKD-FP generates the lowest secondary kinetic energy while having one of the strongest cross-passage pressure gradients in the early part of the passage.

Figures $7.3(\mathrm{e}, \mathrm{f}, \mathrm{g} \& \mathrm{~h})$ show floods of helicity coefficient $\left(C_{H e}\right)$ overlaid with line contours of total pressure deficit coefficient $\left(C_{P_{0}}\right)$. The helicity coefficient quantifies the extent to which an infinitesimal element of fluid in a vortical flow undergoes solid body rotation about an axis parallel to the direction of motion. Helicity is used in the present chapter to examine the intra-passage measurements since it combines the vorticity and streamwise velocity of the secondary flow into a single parameter. Like vorticity, negative helicity has a clockwise sense of rotation. Only the negative helicity is plotted to highlight the passage vortex. The floods of helicity coefficient in Figure 7.3 show that PAKD-FP generates the weakest corkscrew-like motion at the core of the secondary flow, while PAKDF generates the strongest. For PAKD-F, the strong cross-passage pressure gradient in the forward part of the blade row acts on lower momentum fluid. This fluid is thus driven more strongly in the transverse direction and evolves into a stronger secondary flow.

The line contours of total pressure deficit coefficient $\left(C_{P o}\right)$ are typical of intra-passage measurements for turbine cascades with planar endwalls. As was seen in the earlier results chapters, the pitchwise cross-flow near the endwall convects higher energy fluid under the 
a)

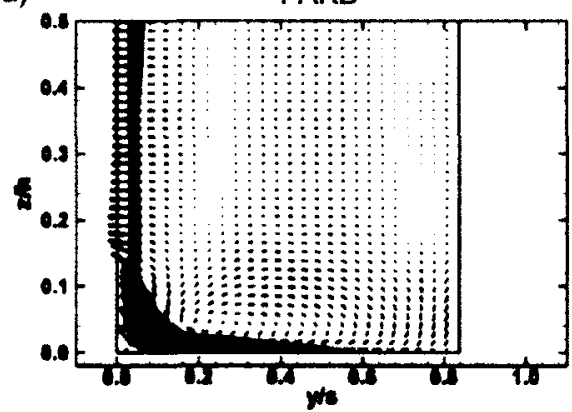

b)

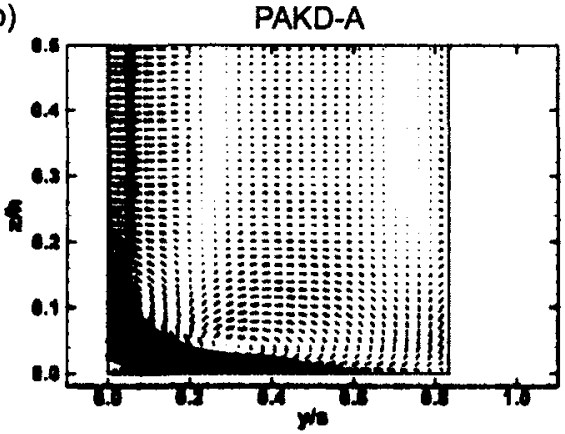

c)

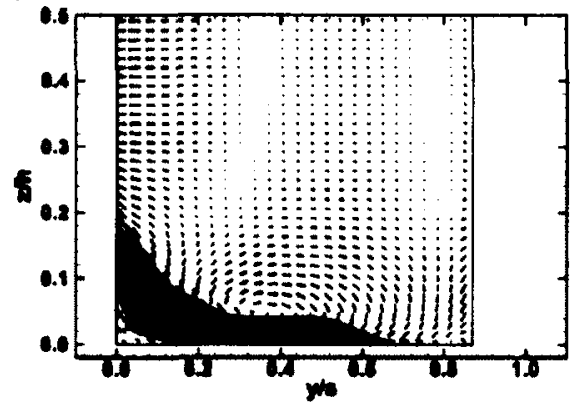

d)

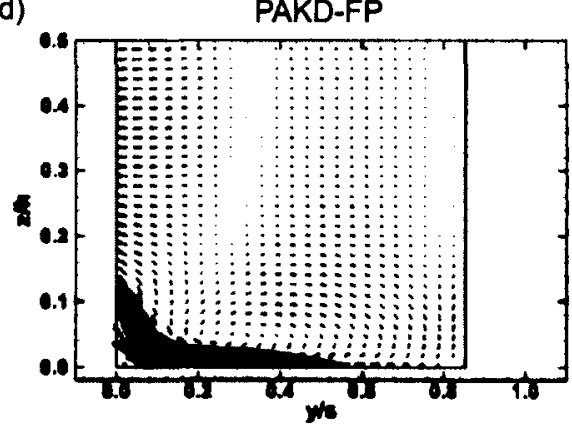

e)

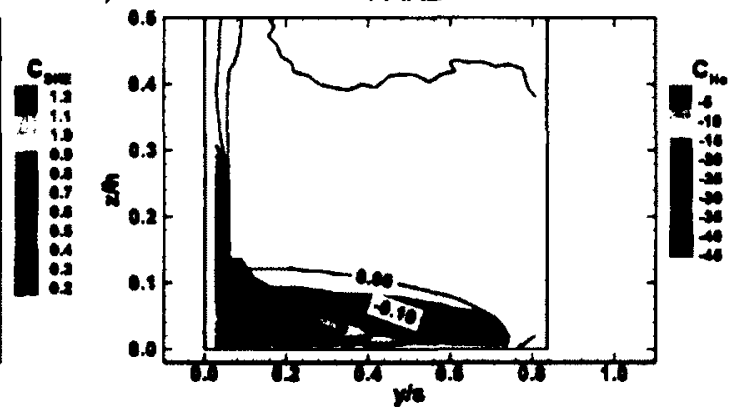

f)

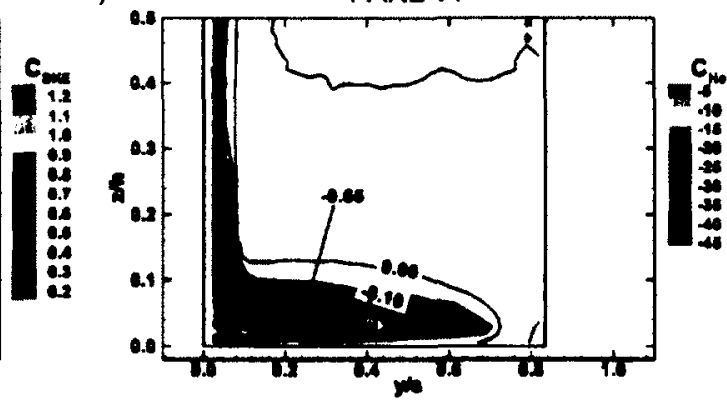

g)

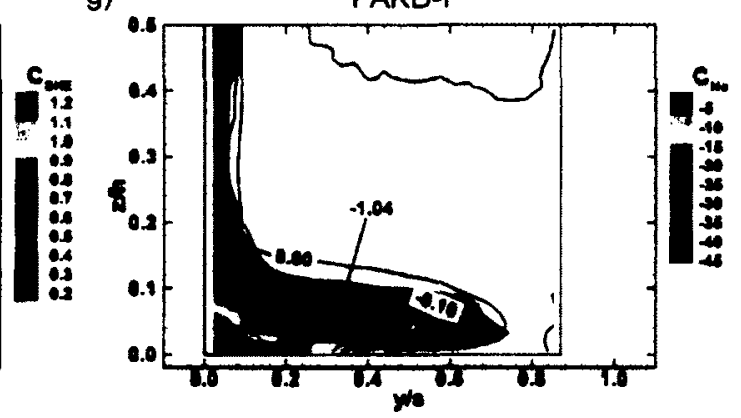

h)

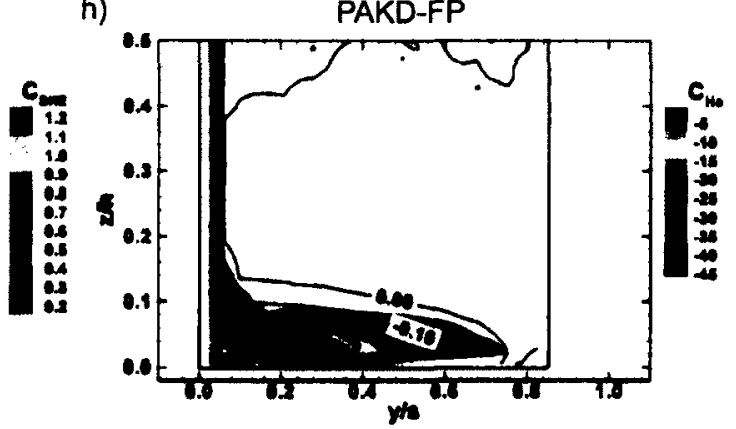

Figure 7.3: Secondary velocity vectors with flood of $C_{S K E}(\mathrm{a}, \mathrm{b}, \mathrm{c} \& \mathrm{~d})$, and line contours of $C_{P o}$ with flood of $C_{H e}(e, f, g \& \mathrm{~h}$ ), at $80 \%$ axial cord for PAKB, PAKD-A, PAKD-F and PAKD-FP 
secondary flow and displaces the passage vortex from the endwall. This effect is more pronounced for PAKD-A and PAKD-F which both generate well defined passage vortices by the $80 \% B_{x}$ measurement plane. PAKD-F develops the largest total pressure deficit at the core of the secondary flow at $C_{P_{o}}=-1.04$ followed by PAKD-A at $C_{P_{o}}=-0.65$. The line contours of $C_{P_{o}}$ for PAKB and PAKD-FP appear similar despite the differences in loading level and loading distribution of the cascades.

The trailing edge plane results are presented in Figure 7.4. The floods of secondary kinetic energy coefficient again show peaks associated with the cross-flow near the endwall and the flow away from the endwall on the blade suction surface. As at the $80 \% B_{x}$ plane, PAKD-FP develops the least secondary kinetic energy in turning the flow through the blade passage. It will be shown in the subsequent downstream planes that this results in less fluid being convected between the passage and counter vortices downstream of the blade row. The flood plots of helicity coefficient reveal that PAKD-F continues to exhibit the strongest passage vortex while PAKD-FP produces the weakest. The line contours of total pressure deficit coefficient for all four cascades show higher energy fluid convected between the passage vortex and the corner vortex, then away from the endwall. This higher energy fluid displaces the loss core associated with the passage vortices of PAKD-F and PAKD-A away from the endwall and the suction surface. The line contours for PAKD-FP and PAKB do not show evidence of a loss core associated with the passage vortex, despite PAKB having a well defined core of negative helicity.

Figure 7.5 presents the data for the first of two downstream measurement planes located at $120 \% B_{x}$. Parts $(\mathrm{e}, \mathrm{f}, \mathrm{g} \& \mathrm{~h})$ show the coefficient of streamwise vorticity, $C_{\omega s}$, rather than the helicity coefficient for comparability with the results of the previous chapter. The passage vortex is identified by the core of negative vorticity. Two counter-rotating (anticlockwise) vortices are also apparent. The corner vortex is found closest to the endwall (approximately $z / h=0.025$ and $y / s=0.05$ ), while the counter-vortex is located above 
a)

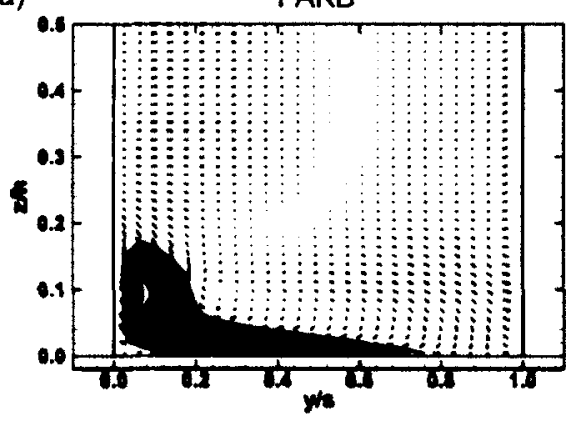

b)

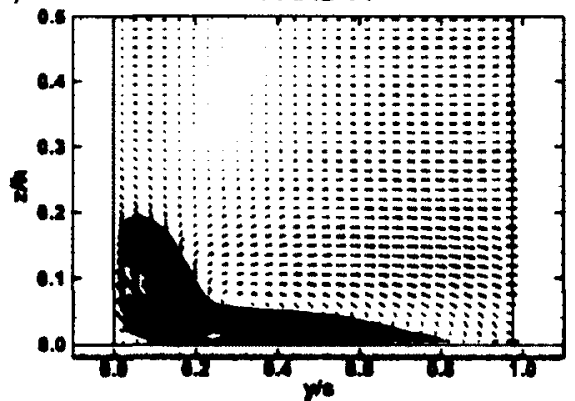

c)

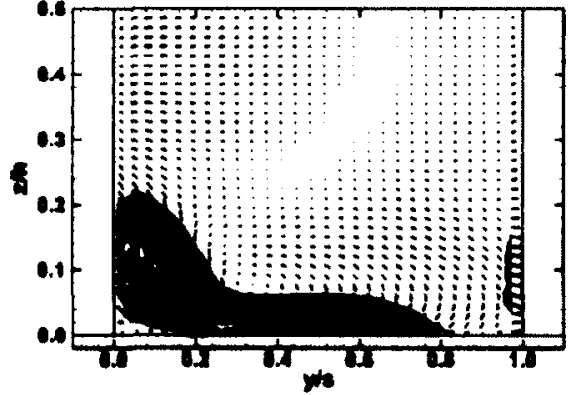

d)

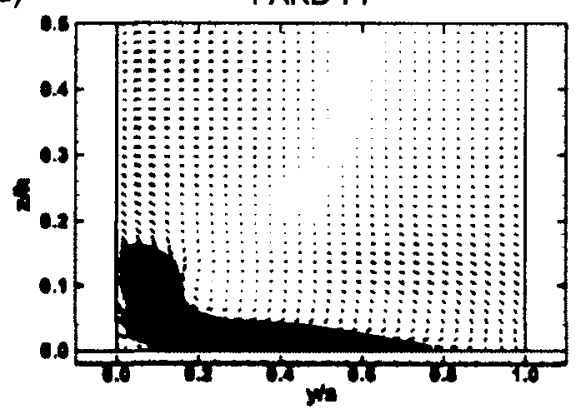

e)

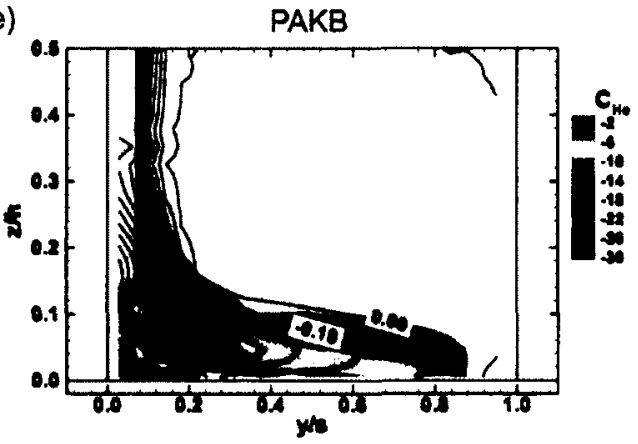

f)

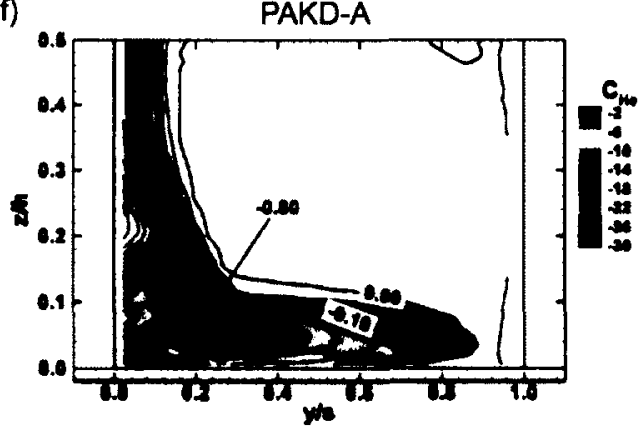

g)

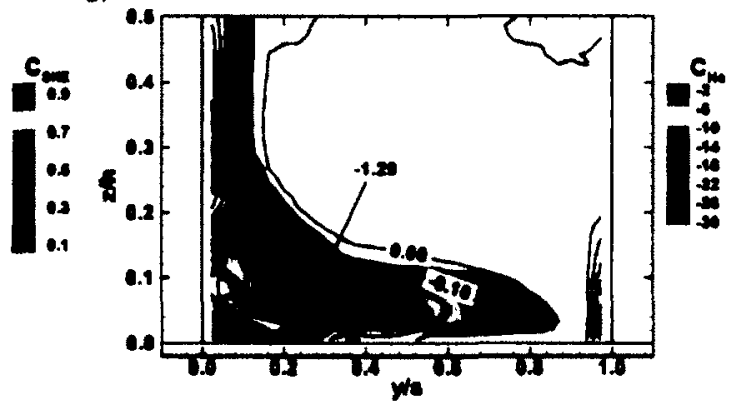

h)

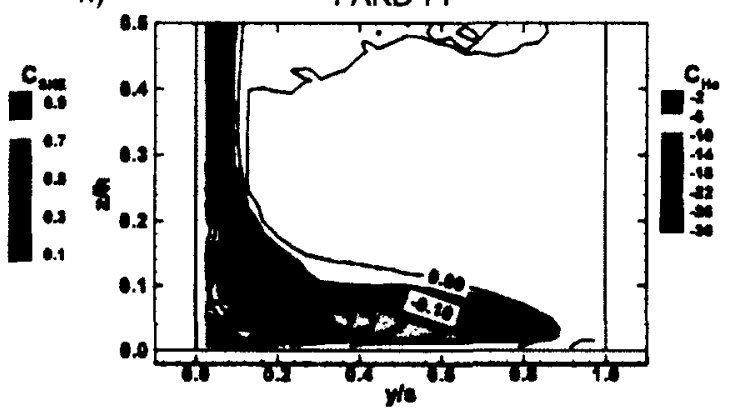

Figure 7.4: Secondary velocity vectors with flood of $C_{S K E}(\mathrm{a}, \mathrm{b}, \mathrm{c} \& \mathrm{~d})$, and line contours $C_{P o}$ with flood of $C_{H e}(e, f, g \& \mathbf{h})$, at the trailing edge plane for PAKB, PAKD-A, PAKD-F and PAKD-FP 
and to the right of the passage vortex. PAKD-FP has the lowest passage vortex vorticity. This is unusual as it is more front-loaded and has a stronger suction peak than the other airfoils tested. Both these features are typically associated with a strong passage vortex. The vorticity through the blade passage is discussed in the following subsection by means of the flow visualizations.

The line contours of total pressure deficit coefficient shown in Figure 7.5 are typical of those produced by linear turbine cascades. There are three loss cores present in the flow fields of all four blade rows. This is in contrast to the trailing edge plane, where the loss cores associated with the passage vortex were indiscernible for PAKB and PAKD-FP. The loss associated with the corner vortex is found nearest the endwall. Next furthest from the endwall, the passage vortex has been indicated with an arrow denoting the value of peak total pressure deficit found at the core. As expected, the front-loaded PAKD-F has the largest secondary flow loss structure with the largest passage vortex total pressure deficit. PAKD-FP has the next largest passage vortex total pressure deficit; however, it has the smallest secondary flow structure of the PAKD airfoils. Further from the endwall, another arrow identifies the third region of higher loss fluid. This fluid is thought to be primarily comprised of suction surface boundary layer fluid that has been displaced by the passage vortex up to the penetration height at the trailing edge. It will be shown in the following subsection that some of the inlet boundary fluid is convected up the suction surface and is incorporated into this loss structure. Higher energy fluid is being convected between this loss core and the passage vortex. The extent to which this occurs is a function of the strength of the secondary velocities driving the convection.

The next plane for the four test cases was measured at $140 \% B_{x}$ downstream of the trailing edge. The results from the exit plane are presented in Figure 7.6. The magnitude of the secondary kinetic energy coefficient $\left(C_{S K E}\right)$ is roughly half of what it was at the $120 \% B_{x}$ plane for all test cases suggesting the secondary flows are diffusing or mixing out. 
a)

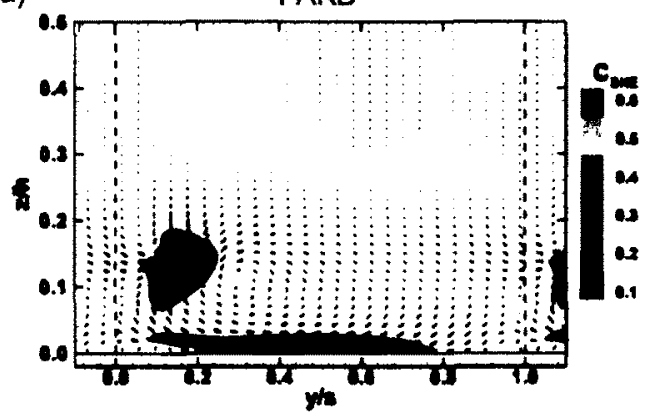

b)

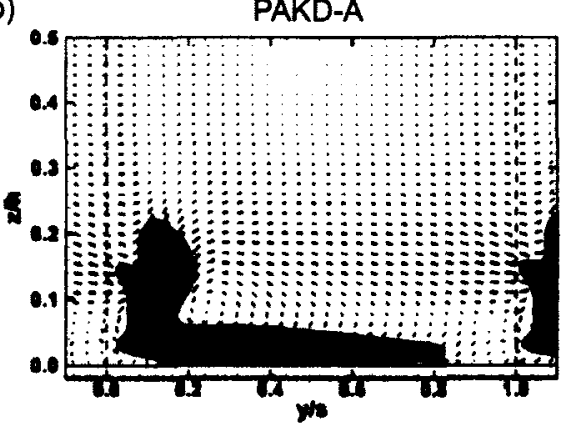

c)

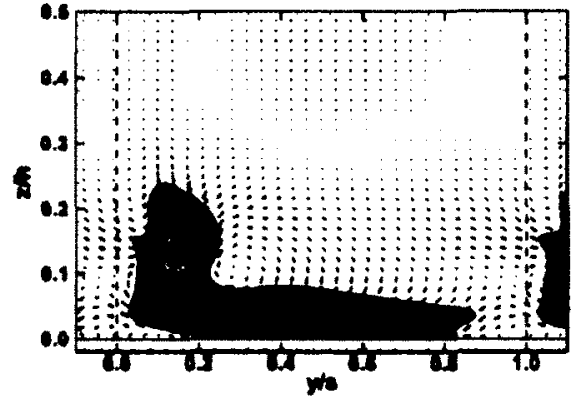

d)

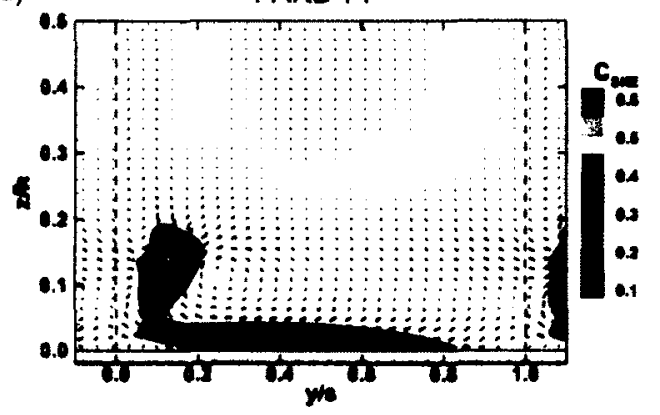

e)

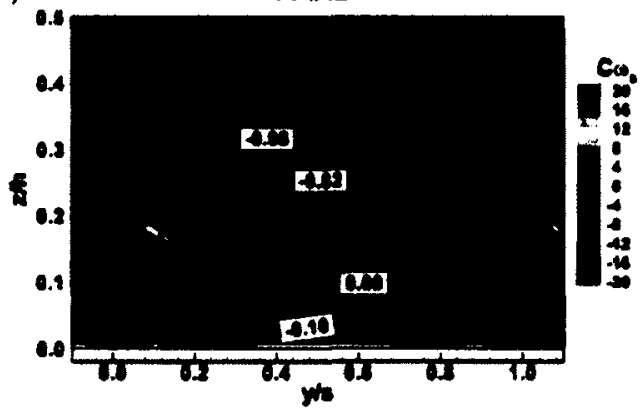

f)

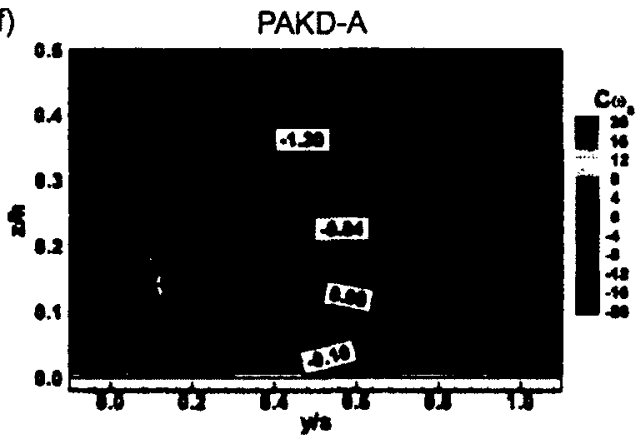

9)

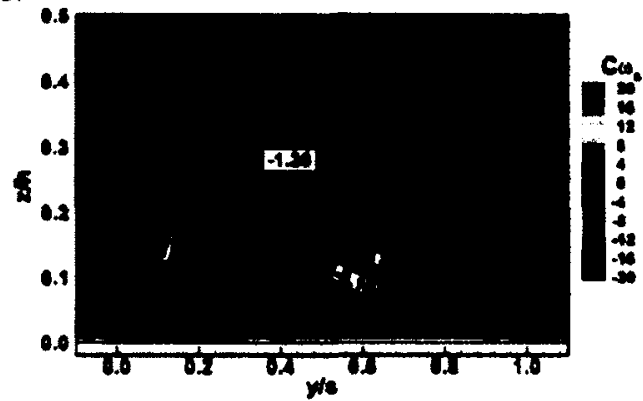

h)

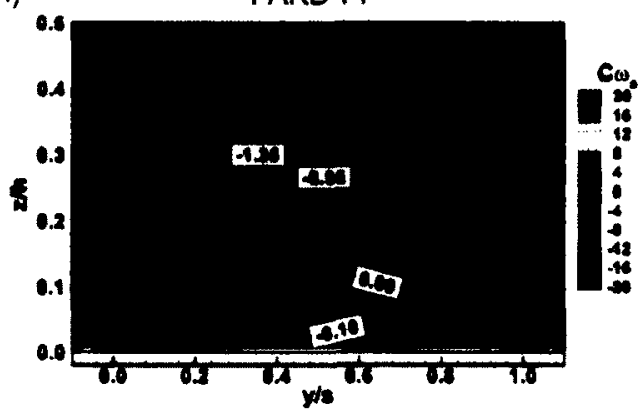

Figure 7.5: Secondary velocity vectors with flood of $C_{S K E}(\mathrm{a}, \mathrm{b}, \mathrm{c} \& \mathrm{~d})$, and line contours of $C_{P_{o}}$ with flood of $C_{\omega s}(\mathrm{e}, \mathrm{f}, \mathrm{g} \& \mathrm{~h})$, at $120 \%$ axial cord for PAKB, PAKD-A, PAKD-F and PAKD-FP 
PAKD-F continues to have the most intense secondary flow. The other test cases have much lower secondary kinetic energy implying that they will incur less additional mixing loss as the flow advances toward the hypothetical mixed-out plane.

The line contours of total pressure deficit coefficient $\left(C_{P o}\right)$ also indicate that the flow fields are diffusing and mixing out. The loss structures in Figures $7.6(\mathrm{e}, \mathrm{f}, \mathrm{g} \& \mathrm{~h})$ are larger than they were at the $120 \% B_{x}$ plane and the peak loss regions identified by the arrows have reduced in magnitude for all but PAKD-F. At the core of the passage vortex for PAKD-F the $C_{P o}$ remains -1.30 . Vortices can typically be approximated as inviscid except at the core where the vorticity is most intense, the velocity gradients are highest, and thus viscous dissipation plays a role. For PAKD-F the dissipation at the core of the passage vortex is severe enough that it dominates the reduction in peak loss resulting from the diffusion. The magnitude of the vorticity coefficient also shows that the structures are diffusing and mixing out. The vortices are larger than they were at $120 \% B_{x}$ and the peak magnitudes (both positive and negative) have reduced.

\subsubsection{Flow Visualization}

This subsection examines the flow fields of the four test cases by means of flow visualization. First, the experimental oil surface flow results are compared to the predicted surface streamlines. The notation of Sieverding (1985) is used to denote the interpreted separation and reattachment lines wherever possible. Following that, the three-dimensional flow field is analyzed by computed streamlines tracked from various heights throughout the inlet boundary layer.

The endwall flow visualizations of all four test cases are presented in Figure 7.7. As in the previous chapters, the flow enters from the right and leaves through the top. The incoming stagnation streamline encounters the initial separation of the boundary layer, $S_{1}$, 
a)

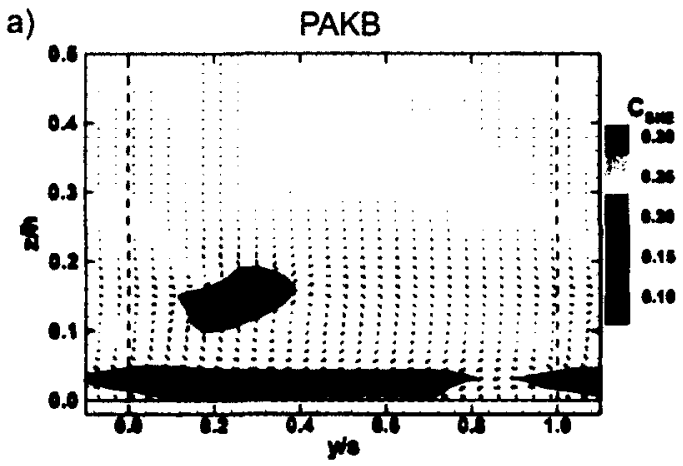

b)

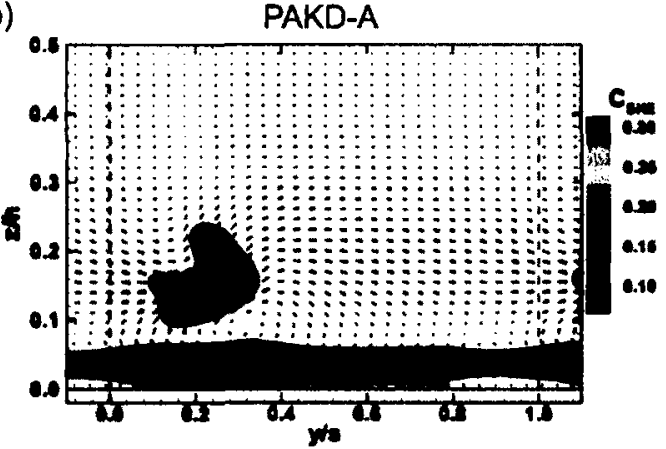

c)

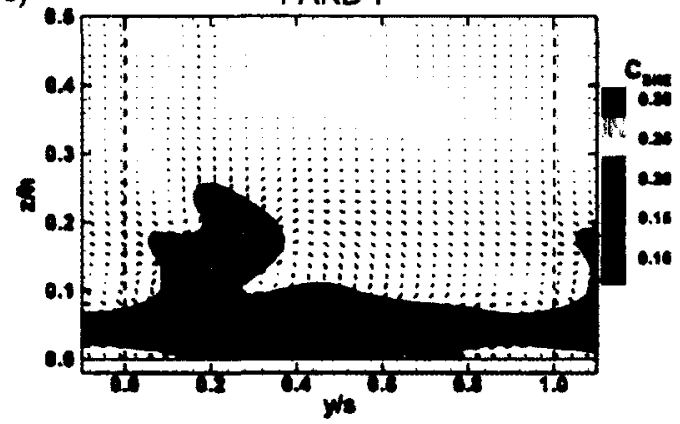

d)

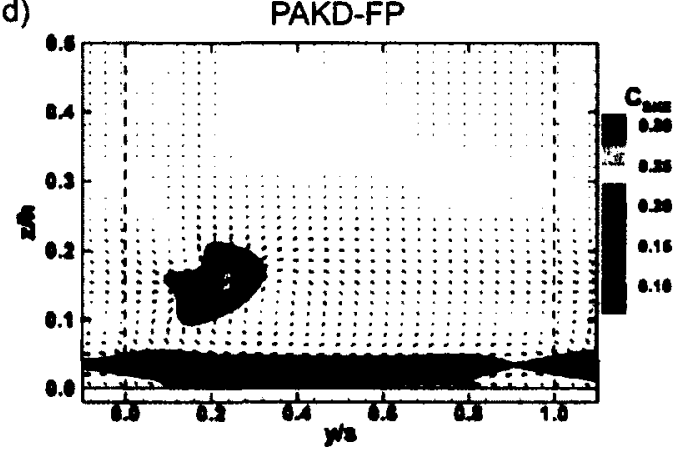

e)

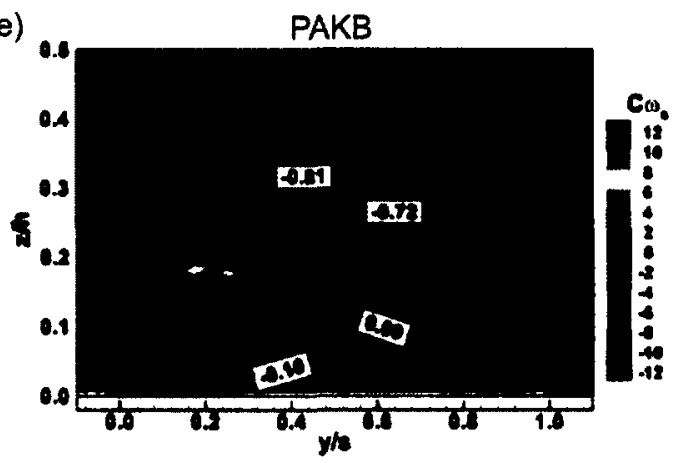

f)

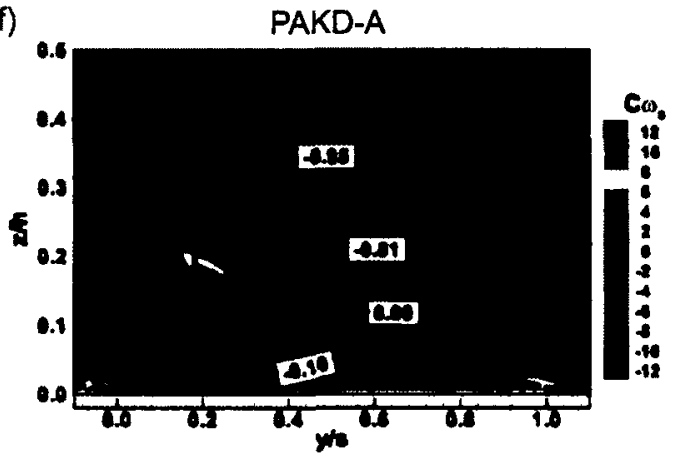

g)

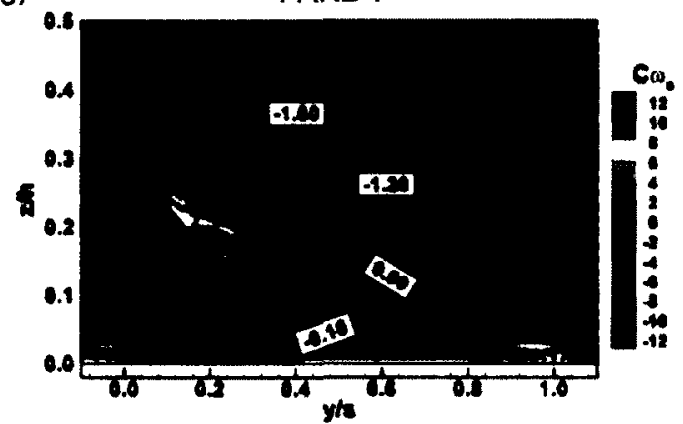

h)

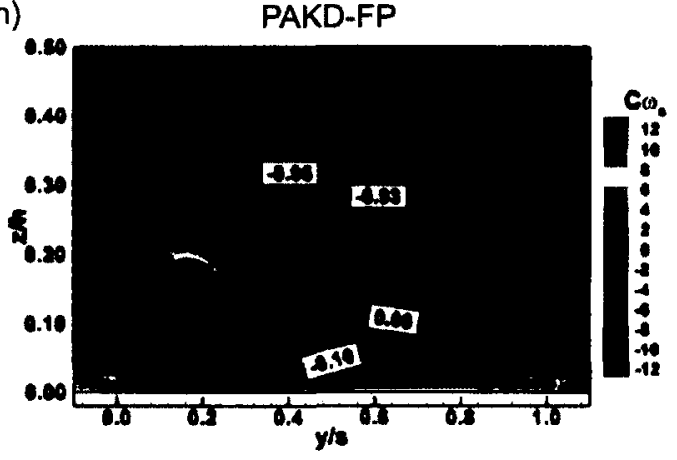

Figure 7.6: Secondary velocity vectors with flood of $C_{S K E}(\mathbf{a}, \mathbf{b}, \mathbf{c} \& \mathbf{d})$, and line contours of $C_{P o}$ with flood of $C_{\omega s}(\mathrm{e}, \mathrm{f}, \mathrm{g} \& \mathrm{~h})$, at $140 \%$ axial cord for PAKB, PAKD-A, PAKD-F and PAKD-FP 
at the saddle point, $A_{1}$. The incoming stagnation streamline marks the division of fluid to either side of the blade passage. Both the experiment and computations show that the saddle point for PAKD-F is further upstream and closer to the suction surface than for the other cascades. This implies that the higher static pressure at the pressure-surface/endwall junction has a stronger potential effect upstream of the blade row. The saddle point for PAKD-FP is closer to the leading edge of the airfoil, but, not as close as those for PAKD-A and PAKB. It appears that forward loading in general drives the saddle point upstream.

Downstream of the initial separation line, the trajectory of the suction and pressure legs of the passage vortex are marked by $S_{2 S}$ and $S_{2 P}$, respectively. The discussion of Figures 7.9 and 7.10 will elaborate on how fluid from different heights in the inlet boundary is fed into the secondary flow structures. Downstream of $S_{2}$ a new boundary layer forms along the endwall. This new boundary layer is driven away from the pressure surface under the influence of the blade-to-blade pressure difference, as highlighted by the arrows in Figure 7.7. The incidence angle that the endwall stream lines encounter $S_{2}$ is indicative of the strength of the passage vortex and the intensity of the helicity. As the acute angle between the surface streamlines and $S_{2 P}$ approaches normal the intensity of the passage vortex increases. In agreement with the measurements presented above, the interaction of the surface streamlines with $S_{2 P}$ suggests that PAKD-F has the strongest passage vortex. Throughout the test cases, the direction of the new boundary layer relative to $S_{2 P}$ is captured with reasonable accuracy by the simulations.

The corner vortex that forms along the endwall junction with the suction surface is marked by the $S_{3}$ separation line. The data of the previous chapter found that the growth of corner vortex was driven by the cross-passage flow into the corner junction. Also, that the size of the corner vortex can be approximated as the distance between $S_{3}$ and the suction surface. As the endwall profiling blends to the nominal wall height, the corner vortex was energized by the resulting overturning of the flow. A similar effect is seen in Figure $7.7 \mathrm{f}$ 
a) PAKB Experiment

b) PAKD-A Experiment

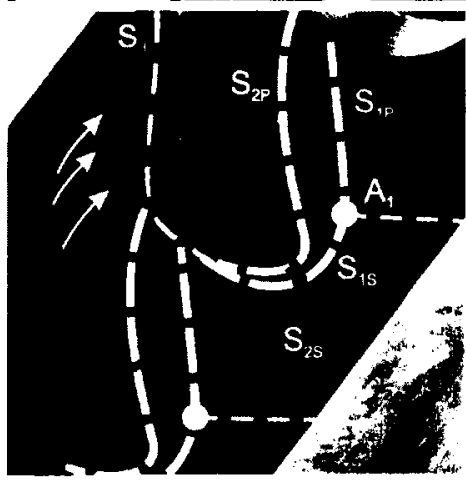

c) PAKD-F Experiment

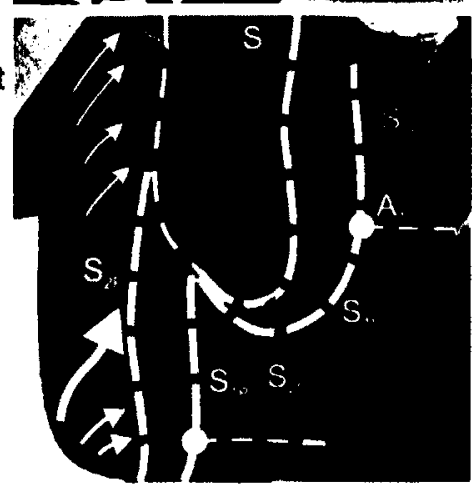

d) PAKD-FP Experiment

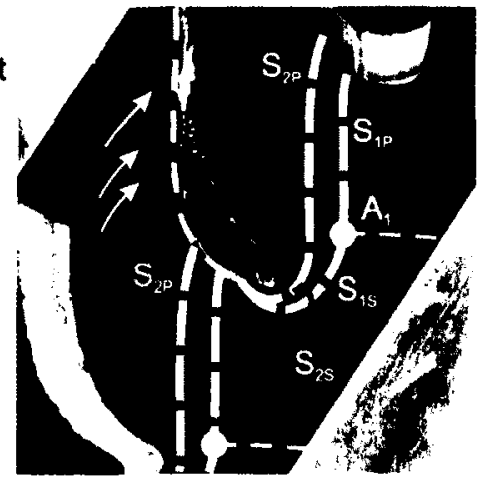

e) PAKB CFD

f) PAKD-A CFD

g)

PAKD-F
CFD
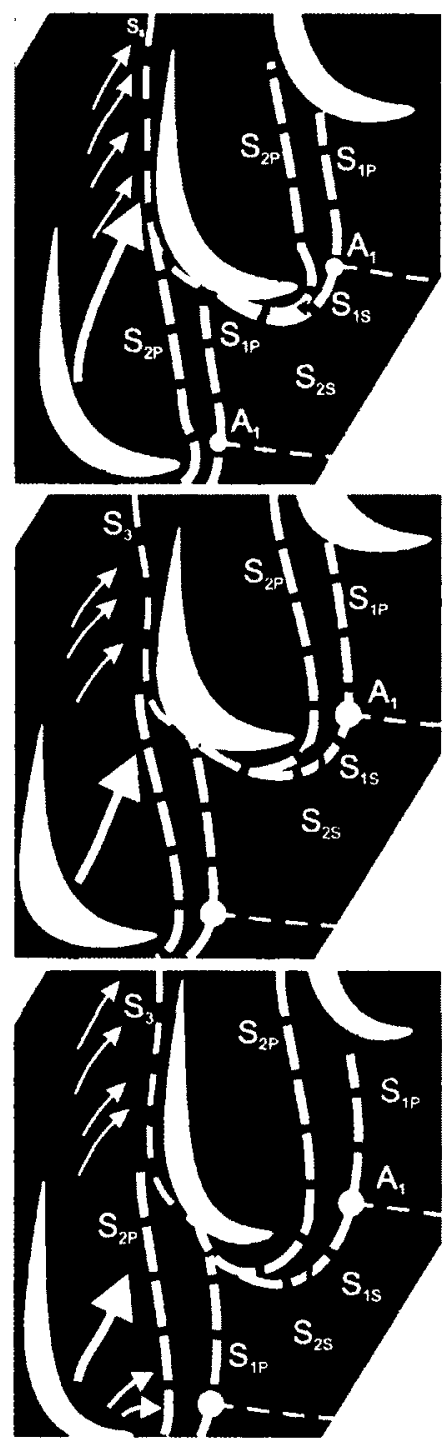

h) PAKD-FP CFD

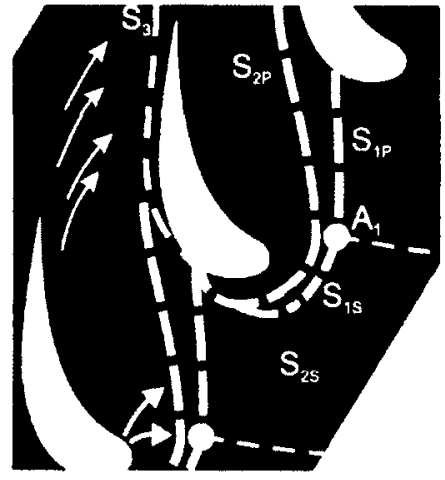

Figure 7.7: Endwall flow visualization for PAKB, PAKD-A, PAKD-F and PAKDFP (Experiment vs. CFD) 
for PAKD-A. PAKD-A has a mild cross-passage pressure gradient in the forward part of the blade passage, followed by a strong suction peak. The inception of the corner vortex is delayed until near the suction peak. Aft of this point the corner vortex grows quickly with downstream distance driven by the strong cross-passage pressure gradient.

The oil surface flow visualizations are compared to the predicted surface streamlines on the suction surface of the cascades in Figure 7.8. The airfoil leading edge is nearest the top and midspan is on the left. The suction surface separation bubble is bound by the $S_{5}$ separation and $R_{5}$ reattachment lines. Predicting the behaviour of transitional boundary layer on the blade suction surface is a challenging task. The computations tend to under predict the streamwise distance required for transition to occur downstream of the laminar separation $\left(S_{5}\right)$ for PAKB and PAKD-A in Figures 7.8e and 7.8f, respectively. As a result, the length of the separation bubble is predicted to be shorter than what is seen in the experiment.

The inception of a separation bubble and the reattachment point is driven by the pressure gradient history the boundary layer experiences and the freestream turbulence intensity and turbulence length scale. However, the Gamma-Theta transition model used in the predictions, uses two transport equations for intermittency and transition onset criteria (in terms of momentum thickness Reynolds number) based on local fluid properties only (Menter et al., 2004). The transport equations do not model the physics of transition, but act as a framework for implementing correlation based models. Instead, the physics of the transition process are captured by the experimental correlations used in the model. The details of the experimental data used in generating the correlation are not given by Menter et al. (2004), but, it is possible that the pressure gradients developed along the suction surface of the PAKD cascades are stronger than those found in the correlation data set ${ }^{1}$.

\footnotetext{
${ }^{1}$ The PAKD airfoils have stronger pressure gradients than those used by Langtry et al. (2004) for validation of the Gamma-Theta transition model.
} 
a) PAKB - Experiment

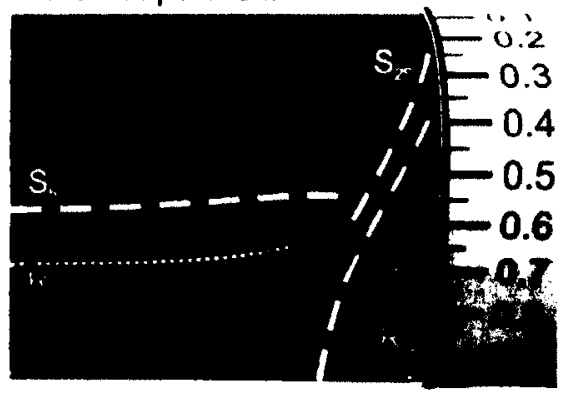

b) PAKD-A - Experiment

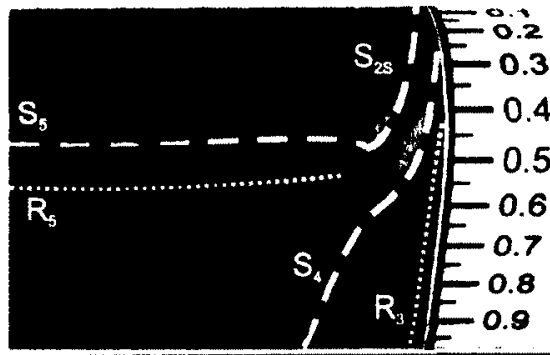

c) PAKD-F - Experiment

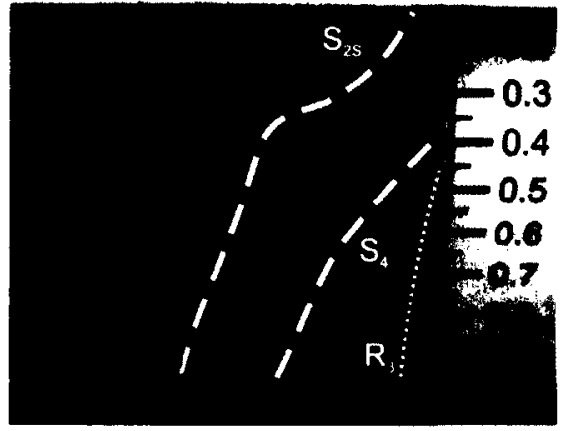

d) PAKD-FP - Experiment

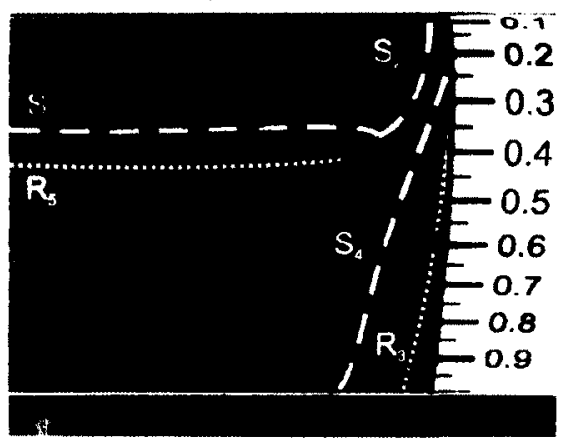

e) PAKB - CFD

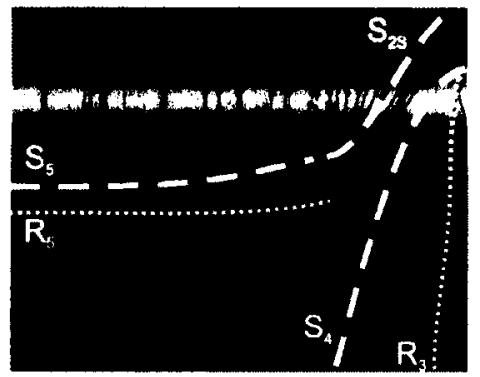

f) PAKD-A - CFD

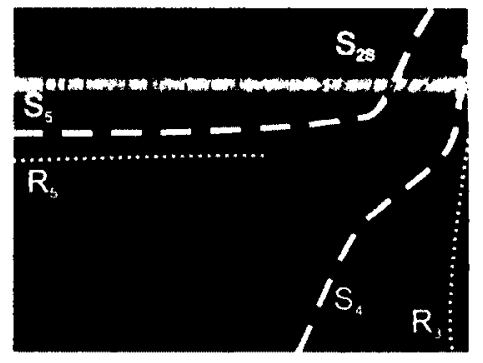

g) PAKD-F - CFD

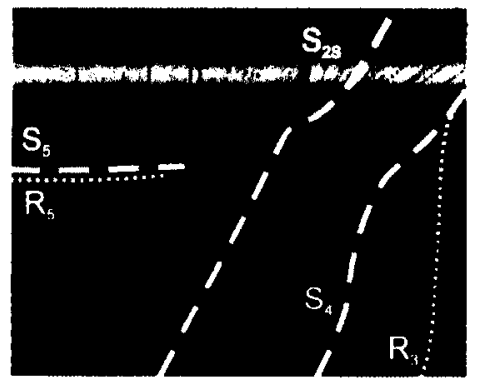

h) PAKD-FP - CFD

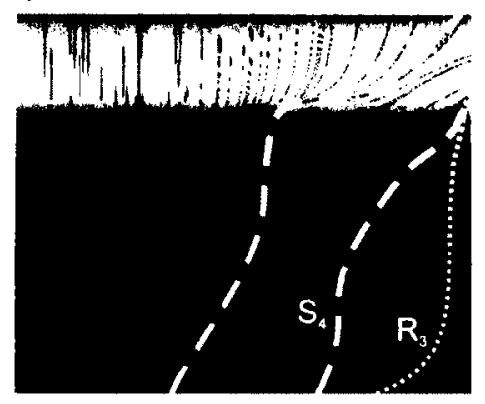

Figure 7.8: Suction surface flow visualization for PAKB, PAKD-A, PAKD-F and PAKD-FP (Experiment vs. CFD) 
It should be noted that the PAKB cascade was used as a test case by Langtry et al. (2004) for validating the Gamma-Theta transition model, albeit the computations were performed with an in-house code developed at the University of Kentucky rather than ANSYS-CFX. The results of Langtry et al. (2004) found that the length of the suction surface separation bubble for PAKB was also slightly under predicted for a Reynolds number of 100,000 based on inlet velocity and axial chord and freestream turbulence intensities of $2.35 \%$ and $6.0 \%$.

It is known that the turbulence intensity specified at the inlet can decay rapidly depending on the inlet eddy viscosity ratio $\left(\mu_{t} / \mu\right)$. This can be avoided by specifying rather large values $\mu_{t} / \mu$ (greater than 100) at the expense of unrealistic levels of skin friction coefficient in the laminar boundary layer near the leading edge (ANSYS-CFX, 2006). The eddy viscosity ratio can be varied by modifying the turbulence length scale for the inlet boundary condition. In the present simulations, the inlet boundary conditions were set per the results of Mahallati (2003) for the turbulence generating grid used; FSTI $=4 \%, \Lambda=$ $0.4 B_{x}$. This led to an inlet eddy viscosity ratio of 27 , and turbulence level at the leading edge plane of $3 \%$, which is in good agreement with the decay in the experiment (Figure 3.10, Mahallati (2003)). For the PAKB simulations presented by Langtry et al. (2004) the inlet freestream turbulence intensities of $2.35 \%$ and $6.0 \%$ had decayed to $1.6 \%$, and $2.85 \%$ at the leading edge of the blade, respectively, suggesting that their inlet $\mu_{t} / \mu$ was set somewhat smaller. The lower turbulence intensity and Reynolds number at the leading edge plane would explain why the simulations of Langtry et al. (2004) predict a slightly longer separation bubble than those of the present work.

The Gamma-Theta transition model identifies a laminar separation by examining the ratio of the vorticity Reynolds number $\left(R e_{v}\right)$ to the critical momentum thickness Reynolds number ${ }^{2}\left(R e_{\theta c}\right)$. If $R e_{v} / R e_{\theta c}$ is large, the intermittency is permitted to exceed 1, although such a value is not physically meaningful for the conventional definition of intermittency. In

\footnotetext{
${ }^{2} R e_{\theta c}$ is the critical Reynolds number where in the intermittency in the boundary layer begins to increase.
} 
regions where the intermittency locally exceeds 1 , the turbulent kinetic energy production grows quickly leading to transition and reattachment. The extent to which this happens in the intermittency equation is controlled by a constant that in theory could be used to manipulate the length of the separation bubble (Menter et al., 2004). In the present simulations a default value of 8 was used but this could be reduced in an attempt to match the separation bubble length shown in the oil surface flow visualization of Figure 7.8a.

The transition model also predicts a small separation bubble for PAKD-F (Figure 7.8g) where none is seen experimentally and fails to predict a rather large separation bubble for PAKD-FP (Figure $7.8 \mathrm{~h})$. The transition momentum thickness Reynolds number $\left(R e_{\theta t}\right)$ is treated as a scalar quantity computed in the freestream based on local turbulence intensity and streamwise pressure gradient. The transport equation diffuses the freestream $R e_{\theta t}$ into the boundary layer where it is required by the intermittency equation. This diffusion is controlled by a coefficient that was obtained based on flat plate transition experiments with streamwise pressure gradients. However, the PAKD cascades see stronger accelerations and deceleration of the flow along the suction surface than what is typically seen in flat plate transition correlations (Mayle, 1991, Roberts and Yaras, 2003, Houtermans et al., 2004. As such, the lag produced by the diffusion control term may not be applicable and the transition location could be miscalculated.

In Figure 7.8, the suction leg of the horseshoe vortex $\left(S_{2 S}\right)$ is seen to migrate up the span of the blade away from the endwall. This effect is well captured by the simulations except for PAKD-FP where the separation bubble is not predicted and $S_{2 S}$ extends to the trailing edge. $R_{3}$ marks the reattachment that bounds the corner vortex from the $S_{3}$ separation line on the endwall (Figure 7.7). The penetration of the reattachment line up the span of the blade appears to be over predicted by the simulations. It should be noted that the precise location of $R_{3}$ is difficult to ascertain experimentally due to the pooling of oil along the junction of the suction surface with the endwall. 
In general, the computations capture the flow physics well. On the endwall the locations of the separation streamlines and their distance relative to one another are predicted reasonably well across the four cascades. Also, the trends associated with the surface streamlines of the new boundary layer forming downstream of $S_{2}$ are seen in the computations as they appear in the experimental oil film visualizations. Although the transition model does not accurately reproduce the physics of the suction surface boundary layer, on the whole the simulations capture the flow field quite well. Based on this, it is expected that the simulations can be used to examine the vorticity in the blade passage.

In classical secondary flow theory, the vorticity associated with the secondary flow is present in the inlet boundary layer and is conserved as that fluid is turned through the blade passage. In the inlet boundary layer, the strongest vorticity exists in the viscous or laminar sublayer. As a result of this, the viscous sublayer presumably contributes strongly to the vorticity of the passage vortex. The vorticity of the inlet boundary layer can be reduced by mixing with vorticity of the opposite sign. Within the blade passage vorticity of the opposite sign to that of the inlet boundary layer is generated in the new boundary layer forming downstream of $S_{2}$ (Figure 7.7) and along the suction surface between the no slip condition at the wall and the secondary flow. Figure 7.9 plots three-dimensional stream traces from the upper edge of the viscous sublayer $(y+=4)$ for PAKD-F (a \& b) and PAKD-FP (c \& d). The stream traces are coloured by the streamwise vorticity coefficient, the component of vorticity in the direction of the exit flow angle at midspan $\left(C \omega_{\alpha, 2}\right)$. For PAKD-F, most of the streamlines from the viscous sublayer find themselves wrapped around the passage vortex where they impart vorticity into the structure. PAKDFP on the other hand sees nearly half of the streamlines from the viscous sublayer migrate along the suction surface where they interact with fluid having vorticity of the opposite sign. These streamlines leave from the trailing edge having no streamwise component of vorticity. 

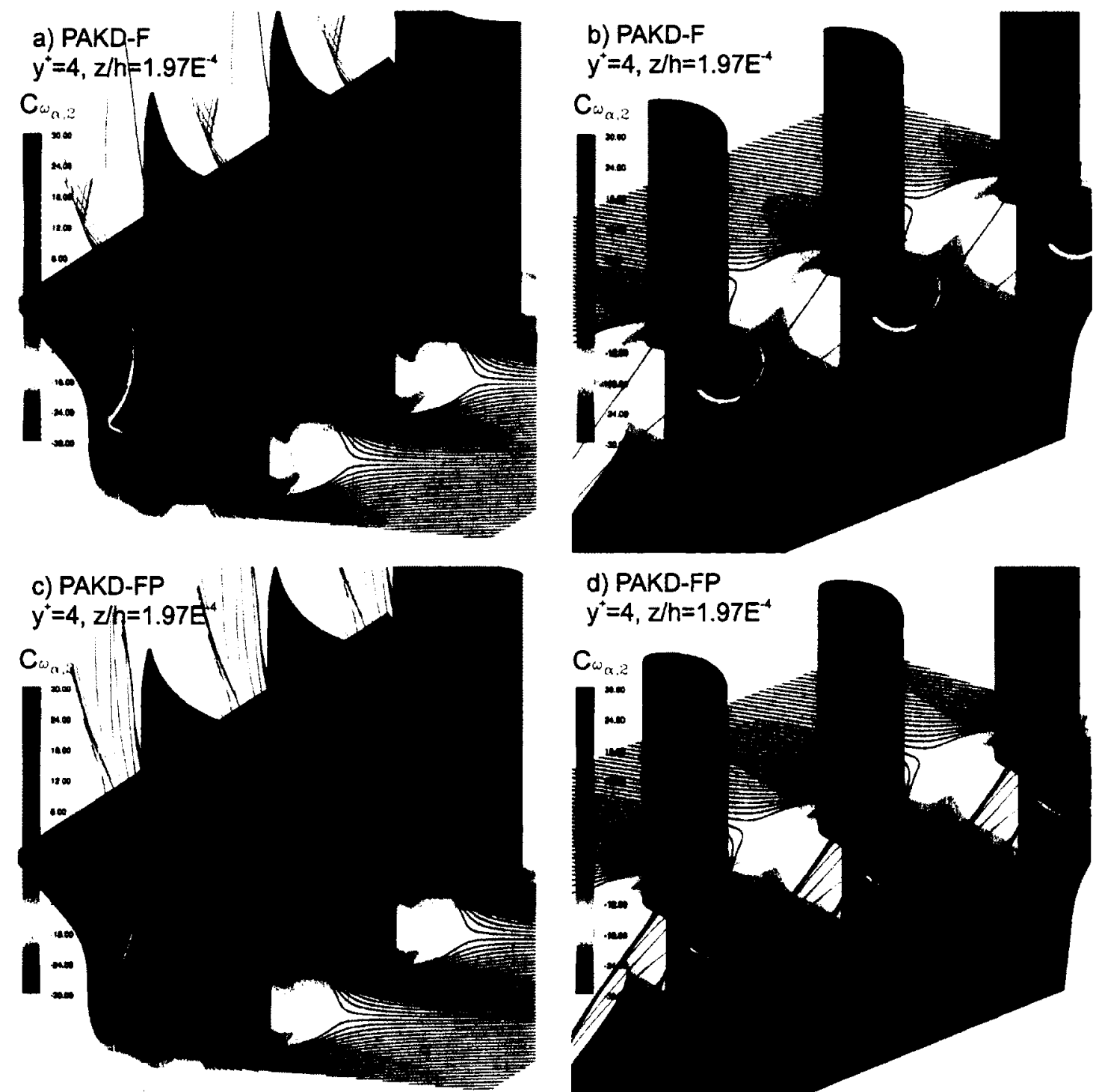

d) PAKD-FP $y^{*}=4, z / h=1.97 E^{-4}$

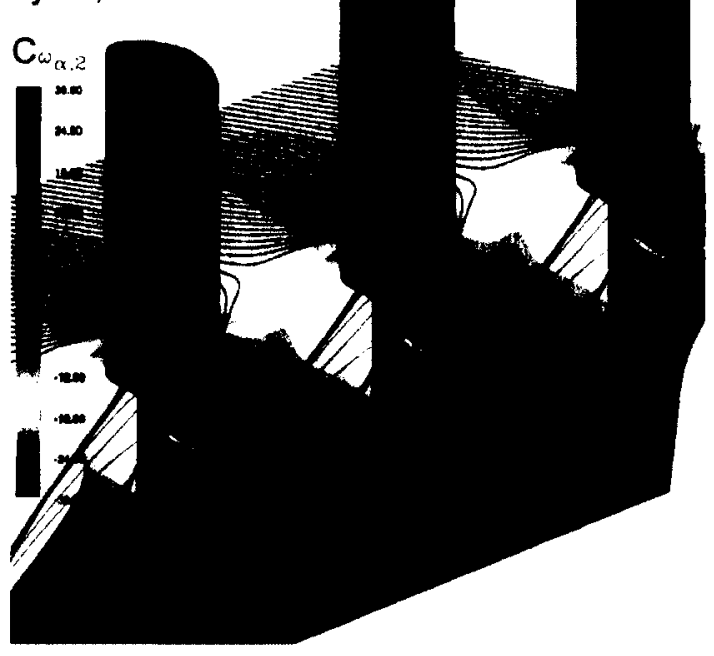

Figure 7.9: Predicted streamlines from $y+=4$ for PAKD-F (a \& b) and PAKDFP (c \& d)

Clearly it is not the viscous sub layer alone, but the entire inlet boundary layer that imparts negative streamwise vorticity into the secondary flow. Figure 7.10 plots stream traces from various heights throughout the law of the wall region of the inlet boundary layer for PAKD-F Figure $7.10(\mathrm{a}, \mathrm{b} \& \mathrm{c}$ ) and PAKD-FP Figure $7.10(\mathrm{~d}, \mathrm{e} \& \mathrm{f})$. For PAKD-FP the fluid from the lower part of the law of the wall region (Figure 7.10d) passes over and wraps around the horseshoe vortex. Some of the streamlines from the same height $(y+=101)$ in 
the inlet boundary layer for PAKD-F appear to stagnate along the suction side leg of the horseshoe vortex (Figure 7.10a). These streamlines are directed in the pitchwise direction for some distance before being enveloped into the secondary flow. This suggests that the horseshoe vortex for PAKD-F is larger than that of PAKD-FP in the saddle point region. 


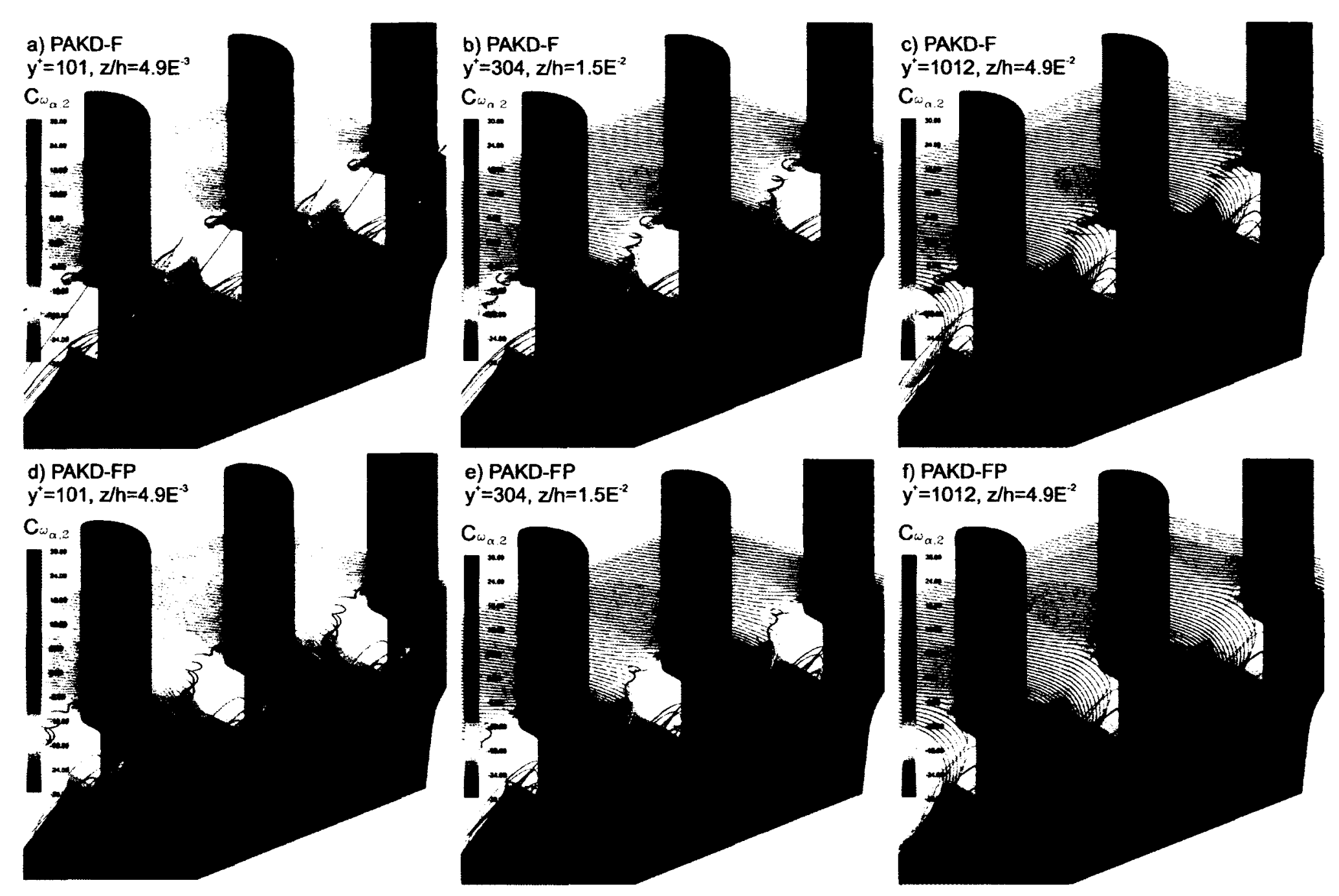

Figure 7.10: Predicted streamlines from the overlap region for PAKD-F (a, b \& c) and PAKD-FP (d, e \& f) 
Farther from the wall in the inlet boundary layer $(y+=304)$ the incoming streamlines have more moderate streamwise vorticity. For both PAKD-F and PAKD-FP, the streamlines that pass along the pressure side of the of blade in Figures $7.10 \mathrm{~b}$ and $7.10 \mathrm{e}$ wrap around the pressure side leg passage vortex and see an increase in $C \omega_{\alpha, 2}$ as they interact with it. For PAKD-F (Figure 7.10b) the streamlines that pass along the suction side of the blade also interact with the passage vortex. On the other hand, in Figure 7.10b some of the streamlines passing along the suction side of the blade avoid being wrapped up by the secondary flow.

Higher still in the inlet boundary layer $(y+=1012)$ the streamlines have negligible vorticity, as expected. All of the incoming streamlines for PAKD-F (Figure 7.10c) have mixed with the secondary flow by the trailing edge plane of the blade passage. The same can be said for PAKD-FP (Figure 7.10f), except that the secondary flow appears to be smaller and some of the streamlines interact with the suction surface boundary layer.

From the discussion of Figures 7.9 and 7.10 it can be seen that the primary difference between the flow fields of the two cascades lies in how the viscous sub layer behaves. Most of the fluid associated with the law of the wall region eventually becomes part of the passage vortex in both test cases. Part of the viscous sublayer for PAKD-FP is drawn up the suction surface of the blade where it mixes with fluid having vorticity of the opposite sign. Consequently, this fluid does not impart strong negative vorticity into the passage vortex. The viscous sublayer is drawn up the suction surface just downstream of the suction peak. Contrary to what would be expected, the strong suction peak in the forward part of the passage results in lower passage-vortex vorticity. PAKD-FP also has higher streamwise momentum than PAKD-F. The higher streamwise momentum in combination with lower streamwise vorticity results in a passage vortex that is more slender and elongated in comparison to PAKD-F. This is seen in Figure 7.11 where streamlines from the top of the inlet boundary layer are plotted for PAKD-F and PAKD-FP. 
In Figures 7.11a and 7.11c the view is in the tangential (pitchwise) direction for PAKDF and PAKD-DP, respectively. The flow enters from the left and out of the page while leaving to the right and into the page. The streamlines for PAKD-FP show an elongated passage vortex in contrast to that of PAKD-F. The view in Figures $7.11 \mathrm{~b}$ and $7.11 \mathrm{~d}$ is into the forward part of the suction surface for PAKD-F and PAKD-DP, respectively. The flow enters from the right and leaves into the page. The passage vortex for PAKD-FP is more slender than that for PAKD-F and is confined closer to the endwall. Again, this is believed to be a result of the higher streamwise momentum and higher helicity entraining less low momentum fluid into the secondary flow. 


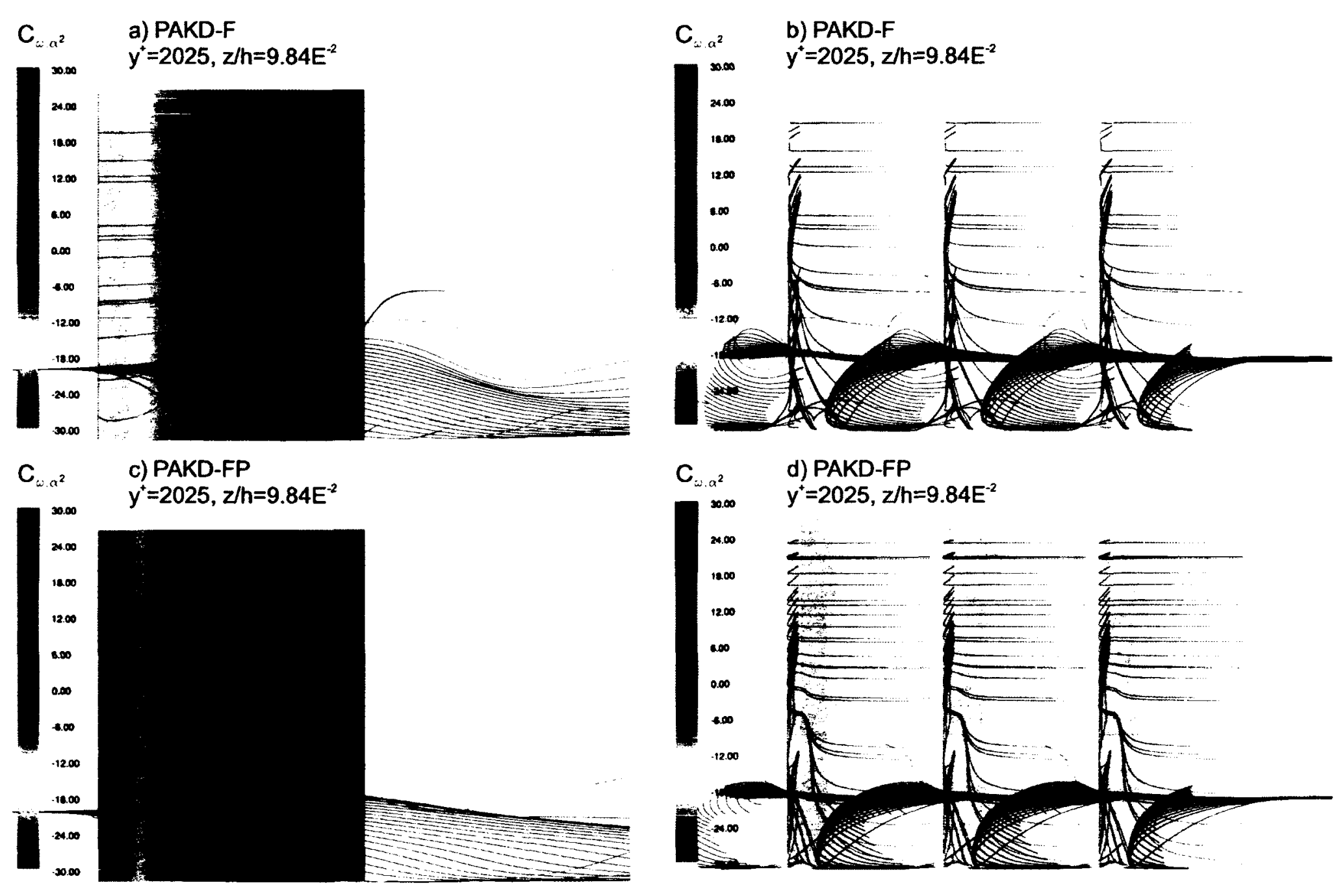

Figure 7.11: Predicted streamlines from $y+=2024$ for PAKD-F (a \& b) and PAKD-FP (c \& d)) 
Figure 7.12 is included in support of the discussion above. The figure shows oil surface flow visualization results for the suction surface of PAKD-FP. The visualization was created by applying pigmented oil only to the endwall of the blade passage. The oil was swept off the endwall just downstream of the suction peak, as was seen with the viscous sublayer in Figures $7.9 \mathrm{c}$ and $7.9 \mathrm{~d}$. Both Figures 7.12 and $7.8 \mathrm{~d}$ show that the location of the suction surface separation bubble marked by $S_{5}$ is relatively unchanged. This alleviates the concern that oil covering the surface might be influencing the flow physics in Figure 7.8. The flow behaviour implied by the oil streaks in Figure 7.12 also demonstrates that the separation line associated with the suction leg of the horseshoe vortex $S_{2 S}$ continues downstream of the separation bubble despite it being difficult to discern in Figure 7.8d. The path of $S_{2 S}$ shows a striking resemblance to the path predicted in Figure $7.8 \mathrm{~h}$ even though the computations fail to predict the separation bubble at all. This behaviour is unexpected as the suction leg of the horseshoe vortex is typically believed to be lifted off the suction surface and wrapped around the passage vortex when $S_{2 S}$ encounters a separation bubble (Sharma and Butler, 1987, Benner et al., 2004b, Sieverding and Van Den Bosche, 1983).

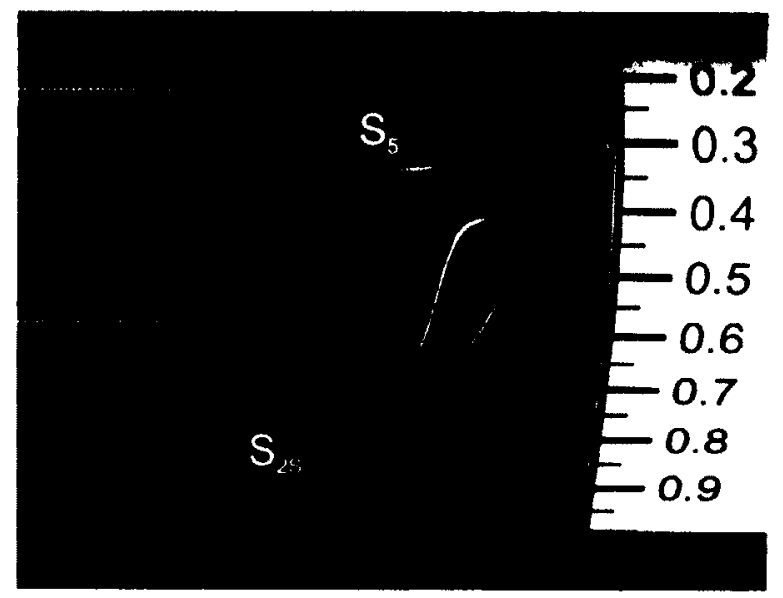

Figure 7.12: Experimental suction surface flow visualization for PAKD-FP 


\subsubsection{Comparison of Computational Predictions with Experimental Data}

This subsection compares the measured and predicted flow fields downstream of the four test cases. The mass-averaged results for the computations are also discussed. The measured and predicted results for the $120 \% B_{x}$ plane are shown in Figure 7.13 . In the figure, contours of streamwise vorticity coefficient $\left(C_{\omega s}\right)$ are overlaid with line contours of total pressure deficit coefficient $\left(C_{P o}\right)$. There are several common trends across the four test cases. The simulations tend to over predict the magnitude of both positive and negative vorticity associated with the secondary flow structures. The vortical structures identified by $C_{\omega s}$ are larger in the computed results than they appear in the measurements. The line contours of total pressure loss coefficient show that the simulations generate higher peak losses as indicated by the arrows. In general the predicted peak loss is trend accurate. The simulations also predict larger loss cores associated with the secondary flow structures. In addition, the secondary flow penetrates further from the endwall than is seen in the measurements. This is in accord with the penetration of $S_{4}$ away from the endwall seen in Figure 7.8 where the computations predict a deeper penetration for all four test cases.

The results at the exit plane at $140 \% B_{x}$ for the four test cases are compared in Figure 7.14. The trends seen here are similar to those discussed for Figure 7.13. The simulations over predict the size and strength of the vortical structures. The peak loss associated with the secondary flow structures and their size is also over estimated by the computations. As before the peak loss indicated by the arrows exhibits reasonable trendwise accuracy across the four test cases. When comparing Figure 7.13 to 7.14 it is observed that the diffusion of the two-dimensional wake and secondary flow structure is captured, at least on a relative scale.

Figure 7.15 presents the pitchwise mass-averaged exit plane results for PAKD-F and PAKD-FP. Note that the line legend is given in Figure 7.15c. The pitchwise mass-averaged 
a)

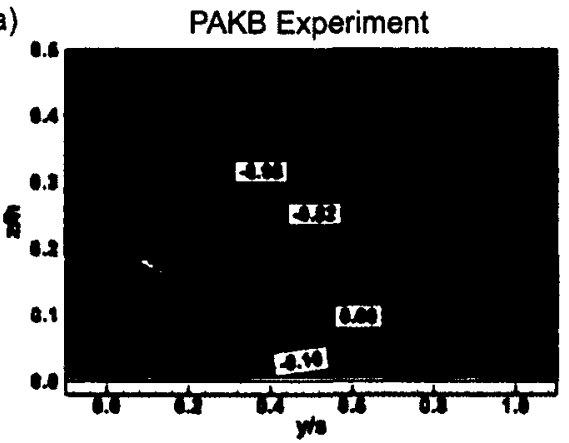

b)

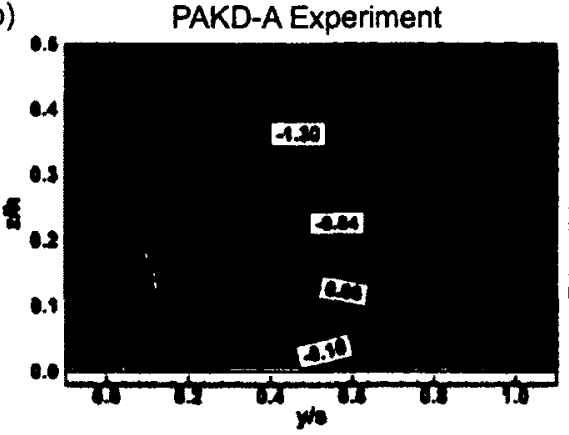

c)

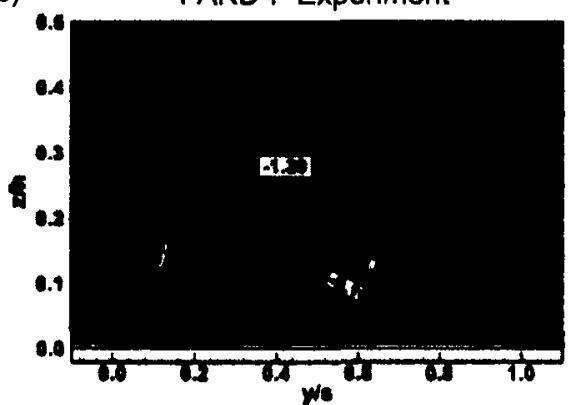

d)

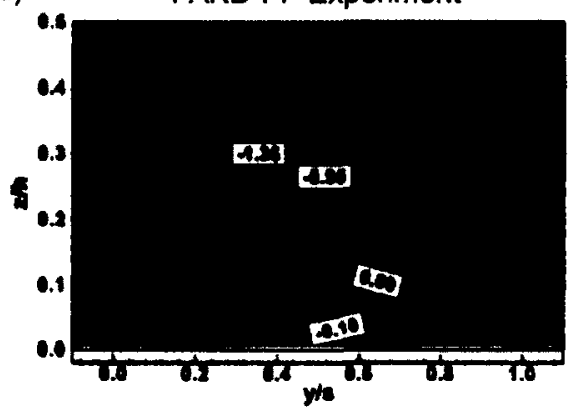

e)

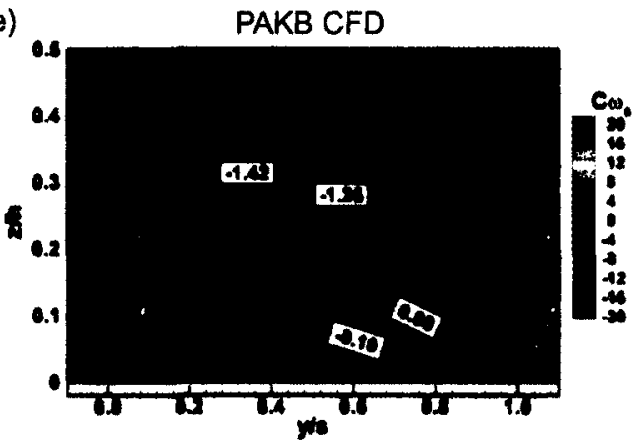

f)

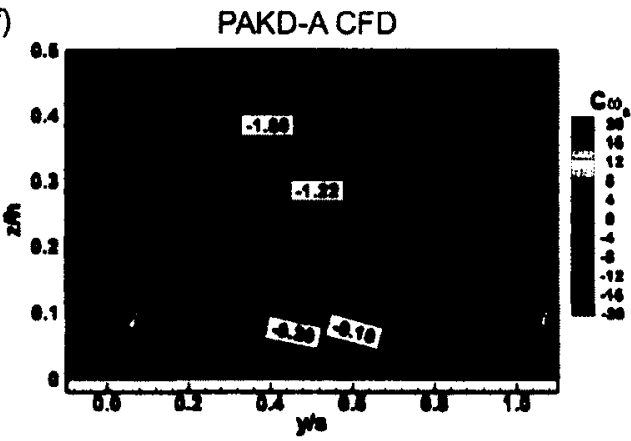

g)

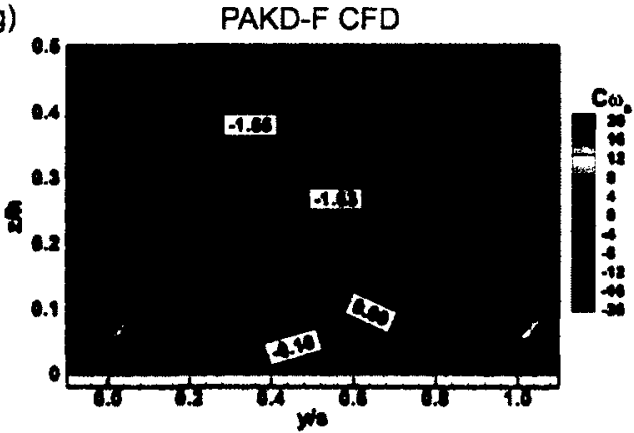

h)

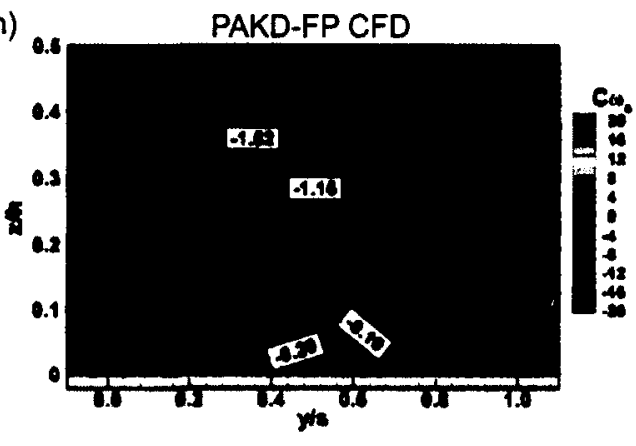

Figure 7.13: Line contours of total pressure loss coefficient $\left(C_{P_{o}}\right)$ with flood of streamwise vorticity coefficient $\left(C_{\omega s}\right)$, Experiment $(\mathbf{a}, \mathbf{b}, \mathbf{c} \& \mathbf{d})$ vs. CFD (e, f, g \& h), at $120 \%$ axial cord for PAKB, PAKD-A, PAKD-F and PAKD-FP 
a)

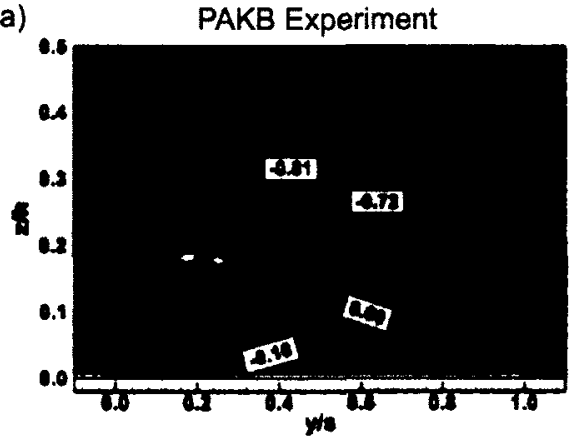

b)

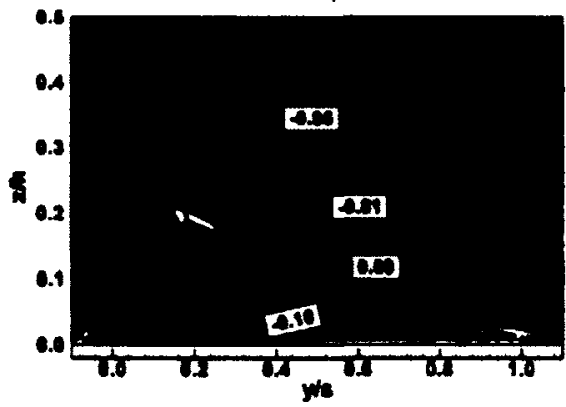

c)

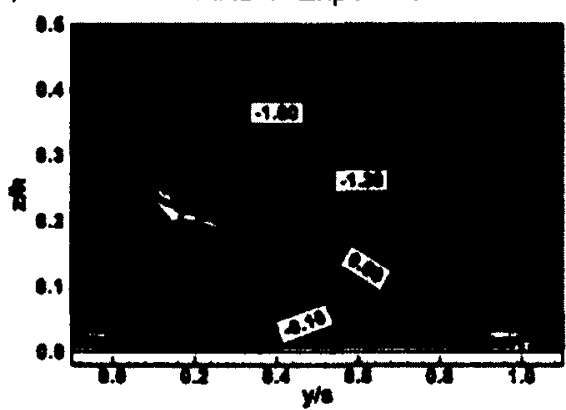

d)

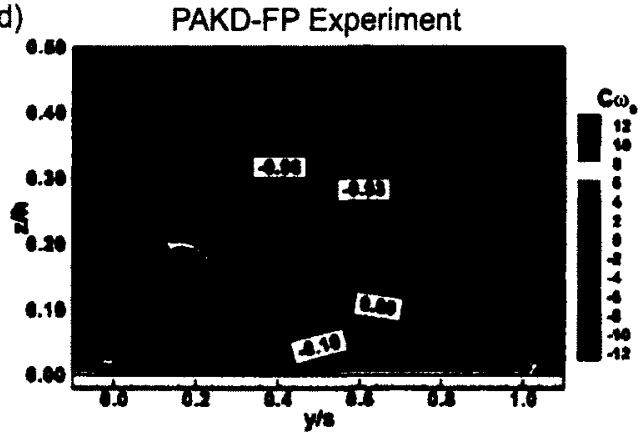

e)

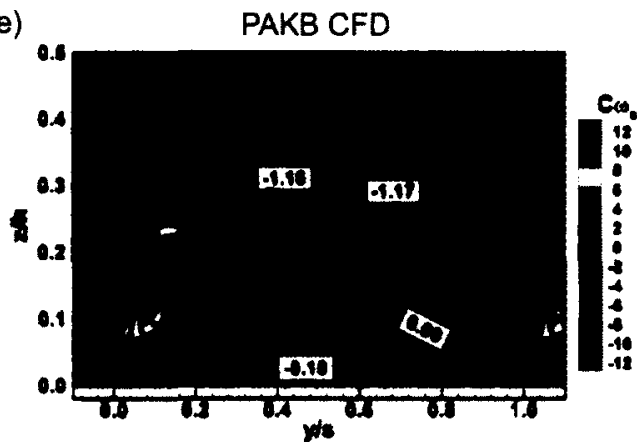

f)

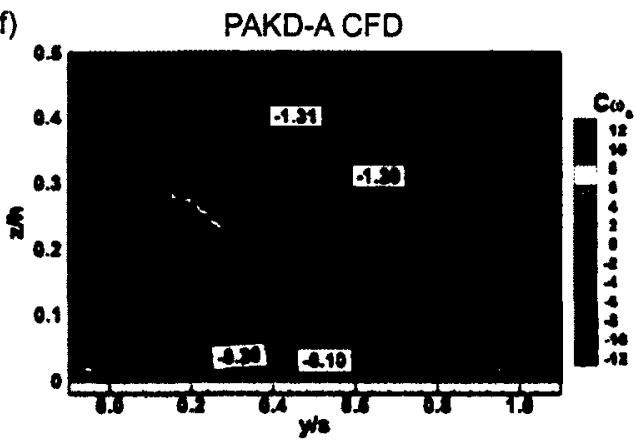

g)

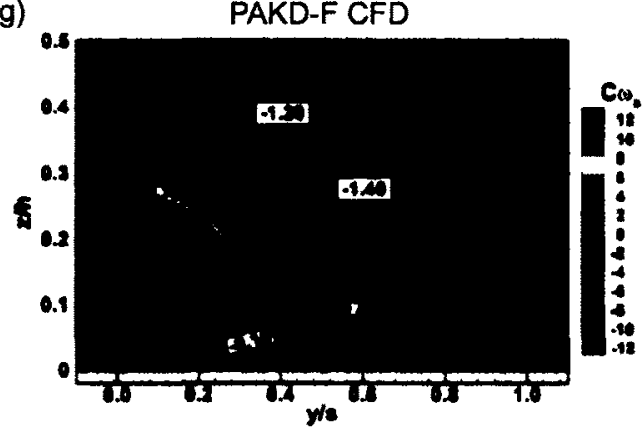

h)

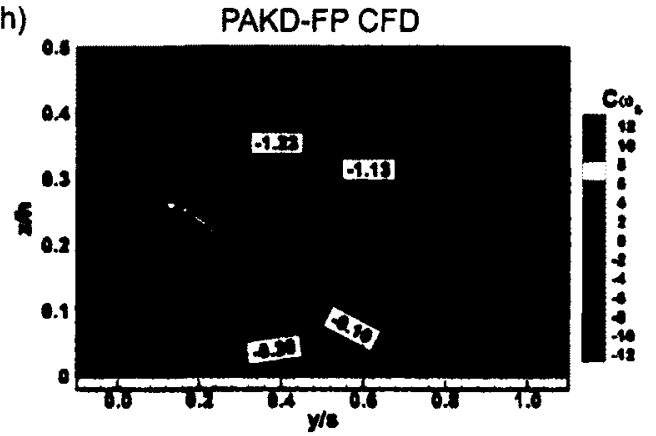

Figure 7.14: Line contours of total pressure loss coefficient $\left(C_{P_{o}}\right)$ with flood of streamwise vorticity coefficient $\left(C_{\omega s}\right)$, Experiment (a, b, c \& d) vs. CFD (e, f, g \& h), at $140 \%$ axial cord for PAKB, PAKD-A, PAKD-F and PAKD-FP 
total pressure loss coefficient is plotted in Figure 7.15a. Throughout the secondary flow, the computations fail to predict the magnitude of the total pressure loss. The number of inflection points in the curves are captured to some degree, but the locations of the inflection points are not. In general the simulations over predict the penetration of the secondary flow toward midspan, as was seen above. Although the simulations do not accurately replicate the physics of the suction surface boundary layers for the two test cases (the presence or absence of separation bubbles of Figures $7.8 \mathrm{c} \& \mathrm{~g}$ and $7.8 \mathrm{~d} \&$ h), the predicted midspan losses compare well with the experiment. This suggests that the influence of the separation bubbles on loss production is small for these particular circumstances. Clearly the loss generation mechanisms of the secondary flow are not being captured. It would be advantageous to document the three dimensional flow with time resolved measurements of the turbulence field. This way the Reynolds stresses, turbulent kinetic energy and dissipation could be evaluated and compared to the computations.

Open literature for linear low-pressure turbine cascades comparing losses predicted with modern CFD methods to experiential data is sparse and the results are inconclusive. For example Hjärne et al. (2007) employed several turbulence closure models within FLUENT to predict the flow around a low pressure turbine outlet guide vane. The results are compared to both five-hole probe and cross-wire hot wire measurements. Downstream of the vane, their predictions using the SST model (the model used in the present work) showed lower vorticity associated with the secondary flow and lower predicted losses, contrary to what is seen above. Hildebrand and Fottner (1999) showed higher predicted losses in the two dimensional wake and secondary flow using both the $k-\epsilon$ and $k-\omega$ turbulence closure models. Cleak and Gregory-Smith (1992) compared cross-wire hot wire and five-hole pressure probe measurements to predictions with various turbulence models. They found that the $k-\epsilon$ model under predicted the turbulence kinetic energy and turbulent viscosity of the secondary flow. Also, the secondary losses were over predicted. For the results of 


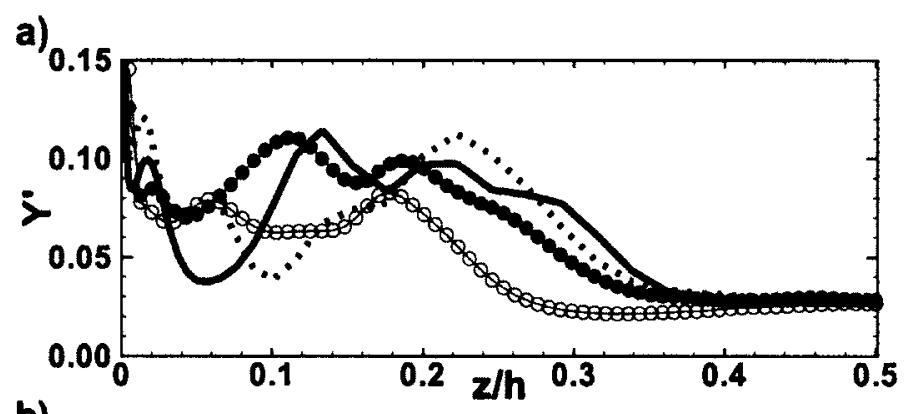

b)

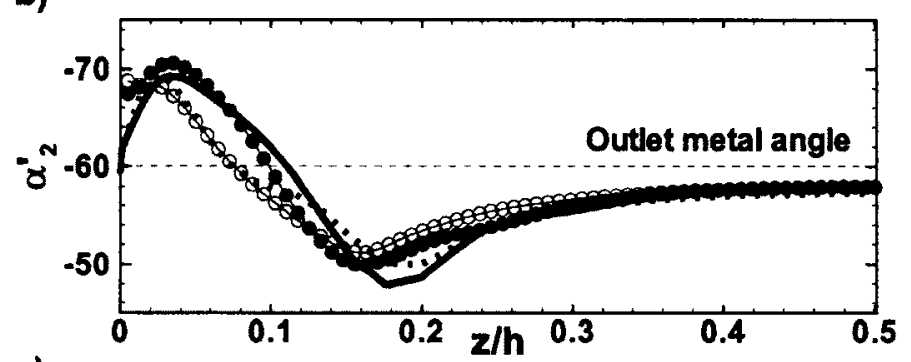

c)

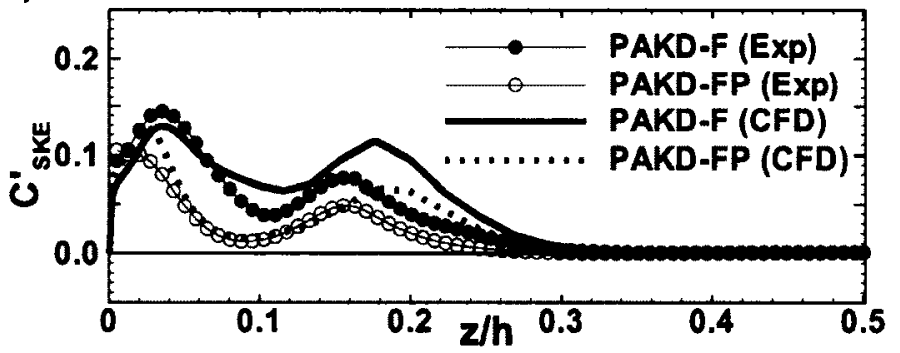

Figure 7.15: Measured and predicted pitchwise mass-averaged results for the $140 \% B_{x}$ plane; Total pressure loss coefficient (a), pitchwise flow angle (b) and secondary kinetic energy coefficient (c)

Hildebrand and Fottner (1999) and Cleak and Gregory-Smith (1992), the over prediction of secondary loss can be partially accounted for by the assumption of a fully turbulent boundary layer on the endwall of the blade passage. It is unclear why the computations of Hjärne et al. (2007) under predict the loss without the implementation of a transition model.

Figure 7.15b presents the pitchwise mass-averaged flow angle for PAKD-F and PAKDFP. The midspan flow angle is predicted accurately. The over-turning of the flow near $20 \%$ span is over estimated by the simulations and so is the under-turning near the wall. 
The secondary kinetic energy coefficient is pitchwise mass-averaged in Figure 7.15c. The secondary kinetic energy is over predicted for both test cases except for in some locations. For PAKD-F between the wall and $7 \%$ span the $C_{S K E}$ is under predicted, whereas the simulations capture the pitchwise mass-averaged $C_{S K E}$ well for PAKD-FP between $6 \%$ and $16 \%$ span.

The exit plane results are tabulated in Table 7.3. The computations do not accurately predict the magnitude of the mass-averaged total pressure loss for the blade row $\left(Y_{\text {total }}\right)$. This is expected from the discussion regarding the line contours of $C_{P_{0}}$ presented in Figure 7.14 and the pitchwise mass-averaged results of Figure $7.15 \mathrm{a}$. The simulations also fail to capture the rank of the four cascades in order of row loss generation $\left(Y_{\text {total }}\right)$. PAKD-A is predicted to have the highest loss generation while it ranks third in Table 7.1. The overall magnitude of mass-averaged secondary kinetic energy coefficient $\left(C_{S K E}^{\prime \prime}\right)$ is over predicted but the rank of the test cases is matched. PAKB and PAKD-FP have similar levels of $C_{S K E}^{\prime \prime}$ while PAKD-A and PAKD-F generate stronger three-dimensional flows with PAKD$\mathrm{F}$ having the strongest. Since the computations capture the trends in $C_{S K E}^{\prime \prime}$ better than those of the blade row loss, it may be beneficial to use the mass-averaged secondary kinetic energy coefficient for the optimization criteria endwall contouring, as suggested by Yan et al. (1990) and Harvey et al. (2000). Even the use of the secondary kinetic energy coefficient must be done so with caution, as it can be seen in Figure $7.15 \mathrm{c}$ that it is not accurately predicted throughout the secondary flow.

The exit flow angle and ratio of outlet-to-inlet dynamic pressure presented in Table 7.3 are well predicted relative to the measured data shown in Table 7.1. The same can be said for the axial velocity ratio and tangential force on the blade. The mixed-out row losses show the same trends as the exit plane row loss discussed above. The mixed-out losses for all four test cases are over predicted. 
Table 7.3: Summary of predicted planar endwall exit plane results for PAKB, PAKD-A, PAKD-F and PAKD-FP

\begin{tabular}{lcrrrr}
\hline LOCATION & FLOW QUANTITY & PAKB & PAKD-A & PAKD-F & PAKD-FP \\
\hline OUTLET & $C_{\text {Pototal }^{\prime \prime}}^{\prime}$ & -0.106 & -0.128 & -0.122 & -0.107 \\
$B_{x}=1.40$ & $C_{P o_{\text {profile }}}^{\prime}$ & -0.063 & -0.078 & -0.063 & -0.066 \\
& $C_{P o_{\text {secondary }}^{\prime \prime}}^{\prime \prime}$ & -0.043 & -0.051 & -0.059 & -0.041 \\
& $Y_{\text {total }}$ & 0.046 & 0.060 & 0.054 & 0.049 \\
& $Y_{\text {profile }}$ & 0.026 & 0.035 & 0.027 & 0.029 \\
& $Y_{\text {secondary }}$ & 0.019 & 0.025 & 0.027 & 0.020 \\
& $C_{S K E}^{\prime \prime}$ & 0.026 & 0.032 & 0.043 & 0.025 \\
& $\alpha_{2}^{\prime \prime}[\mathrm{deg}]$ & -57.2 & -56.1 & -56.8 & -56.9 \\
& $\alpha_{2, m i d s p a n}^{\prime}[\mathrm{deg}]$ & -58.4 & -56.6 & -57.8 & -57.2 \\
& $q_{2}^{\prime \prime} / q_{C L_{1}}$ & 2.341 & 2.181 & 2.312 & 2.272 \\
& $q_{2, \text { midspan }}^{\prime} / q_{C L_{1}}$ & 2.397 & 2.232 & 2.364 & 2.222 \\
& $A V R$ & 0.991 & 1.005 & 1.001 & 0.993 \\
& $F_{Y}$ & 3.48 & 4.17 & 4.28 & 4.23 \\
\hline MIXED-OUT & $C_{P o_{\text {total }}}^{\prime \prime}$ & -0.140 & -0.174 & -0.178 & -0.143 \\
& $Y_{\text {total }}$ & 0.061 & 0.083 & 0.080 & 0.066 \\
\hline \hline
\end{tabular}

CFD has become an essential tool for design and optimization used by a turbine designer. A prudent CFD user would typically establish a baseline simulation case that uses experimental data as a benchmark. This baseline case can then be perturbed in the optimization process in hopes of generating a refined design. The following discussion will outline a potential consequence of this. The results of the simulations have been evaluated as a percent difference relative to PAKB in Table 7.4. The experimentally measured percent difference is also tabulated in parentheses.

Table 7.4: Summary of predicted (and measured) exit plane results, percent change relative to PAKB for PAKD-A, PAKD-F and PAKD-FP

\begin{tabular}{lcrrr}
\hline \hline LOCATION & FLOW QUANTITY & PAKD-A & PAKD-F & PAKD-FP \\
\hline OUTLET & $Y_{\text {total }}$ & $30.7(34.9)$ & $18.5(46.5)$ & $7.3(14.0)$ \\
$B_{x}=1.40$ & $Y_{\text {profile }}$ & $32.3(29.2)$ & $1.5(16.7)$ & $10.5(12.5)$ \\
& $Y_{\text {secondary }}$ & $28.5(42.1)$ & $41.6(84.2)$ & $2.8(15.8)$ \\
& $C_{S K E}^{\prime \prime}$ & $23.9(40.0)$ & $69.8(106.7)$ & $-3.8(13.3)$ \\
& $F_{Y}$ & $19.6(20.9)$ & $23.1(26.0)$ & $21.5(25.7)$ \\
\hline MIXED-OUT & $Y_{\text {total }}$ & $35.7(38.5)$ & $31.1(57.7)$ & $8.8(13.5)$ \\
\hline \hline
\end{tabular}


Analyzing the results as a relative percent difference to PAKB masks the over predicted magnitude of loss and secondary kinetic energy. Table 7.4 shows that the predicted percent difference in row loss $\left(Y_{\text {total }}\right)$ is optimistic in comparison to what was measured experimentally. The same can be said for the profile and secondary losses as well. This is of concern, as a designer may select PAKD-FP as a potential redesign of a stator stage if they were willing to tolerate a $7 \%$ penalty in row loss to achieve a $25 \%$ increase in blade loading. However, in actuality the blade row would suffer twice the predicted increase in loss by the exit plane (14\% as measured experimentally). If compounded over multiple low-pressure turbine stages, this could have significant influence on the overall efficiency of the turbine. A caveat to all these observations is that the flow field of a linear cascade is a highly simplified representation of the flow inside an engine. The complexities of radial pressure gradients, hub purge flow injection, shroud seal flow, tip leakage and unsteady effects from rotor-stator interaction are neglected. The present work also neglects compressibility and temperature effects. Many of these may exacerbate the three-dimensional endwall flow and increase mixing, diffusion and loss generation making them more difficult to predict. Consequently, it is imperative that experimental testing be an integral part of design optimization.

\subsubsection{Discussion on Penetration Depth and Secondary Loss Correlation}

The suction surface oil flow visualizations were presented in Figure 7.8, where the passage vortex separation line was denoted by $S_{4}$. The size of the secondary flow downstream of a blade row is to some extent indicated by the penetration of $S_{4}$ along the trailing edge. This concept of penetration depth was used by Sharma and Butler (1987) to correlate the secondary losses of a turbine passage. The authors related the penetration depth to the axial chord, flow turning, convergence ratio, inlet boundary layer thickness and blade span. By their definition, the three PAKD test cases should have the same penetration depth, which is clearly not the case, as shown by Figure 7.8. The revised penetration depth correlation of 
Benner et al. (2006) has three modifications to that of Sharma and Butler (1987). First it substitutes inlet boundary layer displacement thickness for inlet boundary layer thickness. Second, the flow turning is captured by a tangential loading parameter that decouples the effect of the inlet and outlet flow angles. Third, the blade aspect ratio is incorporated. The final form of the penetration depth correlation of Benner et al. (2006) given by

$$
\frac{Z_{T E}}{h}=\frac{0.1\left(F_{t}\right)^{0.79}}{\sqrt{C R} \cdot(h / C)^{0.55}}+32.70\left(\delta^{*} / h\right)^{2}
$$

where the tangential loading coefficient, $F_{t}$, is

$$
F_{t}=2 \cdot \frac{s}{B_{x}} \cdot \cos ^{2} \alpha_{m}\left(\tan \alpha_{1}-\tan \alpha_{2}\right)
$$

and $\alpha_{m}$ is the vector mean angle through the blade row defined as

$$
\tan \alpha_{m}=0.5\left(\tan \alpha_{1}+\tan \alpha_{2}\right)
$$

But even with these modifications the correlation of Benner et al. (2006) still returns nearly the same value for the penetration depth for all the PAKD airfoils. The mixed-out secondary losses and penetration depths for the four cascades are tabulated in Table 7.5. The table is sorted in order of increasing secondary loss from left to right. The values of $Z_{T E} / h$ returned by Equation 7.2 (Benner et al., 2006) are tabulated along with those inferred experimentally from averaging the penetration depth of a number of suction surface oil flow visualizations. The values of $Z_{T E} / h$ predicted using Equation 7.2 do not successfully capture the trend generated by the PAKD airfoils. Moreover, the experimentally inferred $Z_{T E} / h$ appear to vary proportionally to the secondary losses generated by all four blade rows with PAKB and PAKD-FP having approximately the same value for both.

Based on these results, a parameter that incorporates the effect of loading distribution 
Table 7.5: Summary of secondary loss, penetration depth and quality factor for four cascades

\begin{tabular}{lllll}
\hline \hline & PAKB & PAKD-FP & PAKD-A & PAKD-F \\
\hline Mixed-out $Y_{\text {secondary }}$ & 0.027 & 0.028 & 0.040 & 0.050 \\
$Z_{T E} / h$ (Benner et al., 2006$)$ & 0.13 & 0.16 & 0.15 & 0.16 \\
$Z_{T E} / h$ (Experimental) & 0.12 & 0.12 & 0.15 & 0.16 \\
\hline Quality factor, $Q$ & 5.0 & 9.6 & 7.1 & 7.6 \\
\hline \hline
\end{tabular}

into the correlation of penetration depth may be beneficial. An example of such a parameter is the "quality factor" of Marchal (1980) referenced in the review of Sieverding (1985). The quality factor $(Q)$ is defined as:

$$
Q=\left(\Delta C_{P \max } \frac{q_{C L}}{q_{\text {mean }}} \frac{B_{x}}{s^{*}}\right)^{1.25}\left(1-\frac{x_{\min }}{B_{x}}\right)
$$

where $\Delta C_{P \max }$ is the maximum dimensionless cross-passage pressure difference, $q_{C L}$ is the mass-averaged dynamic pressure at the inlet centerline, and $q_{\text {mean }}$ is the vector mean of the inlet and outlet mass-averaged dynamic pressures at the centerline. The cross passage distance at the location of the suction peak is denoted by $s^{*}$, and $x_{\min }$ represents the axial distance downstream of the leading edge to the suction peak.

Marchal (1980) proposed that an increase in the blade-to-blade pressure difference or a shift in the loading distribution toward the leading edge would produce higher secondary loss. His hypothesis was confirmed by Weiss and Fottner (1995), Benner et al. (2004b) and more recently by Zorić (2006) for the earlier PAK airfoils (PAKB, PAKD-A, PAKD-F). The quality factor for the four cascades is included in Table 7.5. The results here confirm the findings of Zoric (2006) who determined that the quality factor effectively ranks the secondary losses for PAKB, PAKD-A and PAKD-F from lowest to highest. However the quality factor does not properly scale the secondary losses of PAKD-FP which has the largest value of $Q$, but generates the second lowest secondary loss.

The discussion related to Table 7.2 suggested that the minimum value of $V_{\text {local }} / V_{1}$ 
occurring on the pressure-surface, downstream of the acceleration from the stagnation point, is successful at ranking the secondary losses generated by the four cascades tested. This minimum $V_{\text {local }} / V_{1}$ could be used to formulate a modified correlation for penetration depth and secondary loss. Such a modified correlation might incorporate the axial location and blade-to-blade distance at the location of the minimum $V_{\text {local }} / V_{1}$. Further investigation is required incorporating the results of other studies to determine if the streamwise momentum of the flow has a similar effect to that seen above on the roll-up of the passage vortex and subsequent secondary loss production.

\subsection{Conclusions}

This chapter has demonstrated the potential to employ highly-loaded turbine airfoils in a linear cascade without substantial penalty in blade row loss. A baseline test case having a Zweifel coefficient of 1.08 was compared to three cascades having $25 \%$ higher blade loading. All test cases had the same velocity triangles. The highly loaded cascades had varied loading distributions; these distributions were aft-loaded, forward-loaded, and a novel forward-loaded approach that maintains an elevated streamwise momentum of the flow. This modified-loading approach was found to be effective for secondary loss reduction relative to the other highly-loaded test cases. The modified-loading approach generated slightly higher row loss than the baseline case, but could be an attractive replacement given the potential for weight reduction.

Complimentary RANS CFD simulations were performed for each of the test cases. The computations replicated the flow physics with reasonable accuracy. The Gamma-Theta transition model was implemented to predict transition on the endwall and blade surfaces. The profile losses were well predicted despite the inability to predict presence or absence of midspan separation bubbles in two of the cases. This suggests that the influence of the 
separation bubbles on loss production was small for these particular circumstances. The secondary losses and secondary kinetic energy are over predicted for all four cascades. The computations fail to predict the rank of the secondary losses, but the rank of the secondary kinetic energy is correctly computed.

In general it was found that the modified-loading approach generated levels of secondary kinetic energy and secondary loss that are comparable, but somewhat higher, to that of the baseline case. The modified-loading approach produced a weaker and smaller passage vortex than the other highly-loaded cases. The weaker passage vortex was attributed to the behaviour of the viscous sublayer which was drawn up the suction surface of the blade where it interacts with fluid having opposing vorticity. The weaker streamwise vorticity, in combination with higher streamwise momentum, resulted in a passage vortex that entrained less fluid into the secondary flow structure. The end result was a passage vortex that was more slender and remained closer to the endwall relative to those of the other highly-loaded cases.

Some concerns have been raised as to the ability of correlations in open literature to predict the penetration depth of the secondary flow. It has been shown that the penetration depth, and the secondary losses generated are inversely proportional to the extent of nearstagnation that occurs locally on the pressure surface of the blade. That is, the minimum $V_{\text {local }} / V_{1}$ that occurs on the pressure-surface, downstream of the acceleration from the stagnation point, could perhaps be used to formulate a modified correlation for penetration depth and secondary loss. The results of other studies should be investigated to determine if an elevated level of streamwise momentum has a similar effect to that seen in the current data set.

This chapter has shown that significant reduction in secondary loss can be achieved through modification of the airfoil loading distribution. The loss reduction achieved by the 
modified-loading approach is greater than that which was seen for the application of nonaxisymmetric endwall contouring in the previous chapter. In addition it does not require the added machining complexity of a profiled endwall. Thus, the modified-loading approach is attractive from the perspective of an engine weight reduction strategy. One could also envision combining the modified loading approach with endwall contouring to determine if synergistic effects could be found. 


\section{Chapter 8}

\section{The Effect of Blade Loading on}

\section{Losses in an Extremely}

\section{Highly-Loaded Linear Turbine}

\section{Cascade with Planar Endwalls}

\subsection{Introduction}

Conventional lift turbine airfoils are those having a Zweifel coefficient $\left(Z_{w}\right)$ of $0.7-0.8$. The baseline airfoil in this thesis, PAKB has a design Zweifel coefficient of 1.08. The term highly-loaded is open to interpretation and various research groups have developed their own terminology. For example Zhang and Hodson (2005) consider the T106C airfoil, having a Zweifel coefficient of 1.2 to be an "Ultra-High-Lift" low-pressure turbine airfoil. By comparison, the PAKD generation of airfoils presented in previous chapters have a design Zweifel coefficient of 1.36 . In this chapter the results for an extremely-highly-loaded airfoil 
are discussed. This airfoil, designated PAKF, has a design Zweifel coefficient of 1.78 .

Previous to this work, the most highly loaded cascade data in the open literature was that of Houtermans et al. (2004) who described the low-speed redesign and testing of a low-pressure turbine vane. Their cascade, having a Zweifel coefficient of 1.473 , was tested

over a Reynolds number range of $5 \times 10^{4}$ to $2 \times 10^{5}$ based on exit velocity and blade chord at a relatively low level of freestream turbulence $(0.6 \%)$. The authors' discussion is focussed primarily on correlating the dynamics of the suction surface separation bubble, although some profile and secondary loss data is presented.

Highly loaded turbine blades are notorious for having high losses and for experiencing fully separated stall at low Reynolds numbers. It will be shown in this chapter that it is possible to design an extremely highly loaded cascade that has comparable midspan performance to the baseline blade row used in this study (PAKB). It will also be shown, however, that the extremely highly loaded cascade generates strong secondary flows and higher secondary loss relative to the other airfoils tested. The chapter concludes with a discussion on expanding the design envelope by examining the losses generated by the various PAK airfoils.

\subsection{Results and Discussion}

The results of this chapter span a Reynolds number range of $2.5 \times 10^{4}$ to $1.35 \times 10^{5}$ based on inlet velocity and axial chord. Unless otherwise stated the results presented are at a Reynolds number of $1.26 \times 10^{5}$. 


\subsubsection{Midspan Performance}

Figure 8.1 shows the predicted midspan loading distribution for the PAK family of airfoils in terms of the local isentropic velocity relative to the velocity on the inlet centerline of the test section. The predictions are validated by experimental results for two of the test cases measured by the present author. In general the predictions are in good agreement with the measured results. PAKF is forward loaded, with the suction peak occurring near $35 \%$ axial chord, though not as forward loaded as PAKD-F or the redesigned PAKD-FP. Along the aft portion of the suction surface (downstream of $55 \%$ axial chord) PAKF has a pressure distribution that is similar to that of PAKD-A. PAKF has lower velocity along much of the pressure surface in comparison to the other cascades used in this study. The effect of the loading distribution will be discussed in regard to the profile losses generated when examining Figure 8.5.

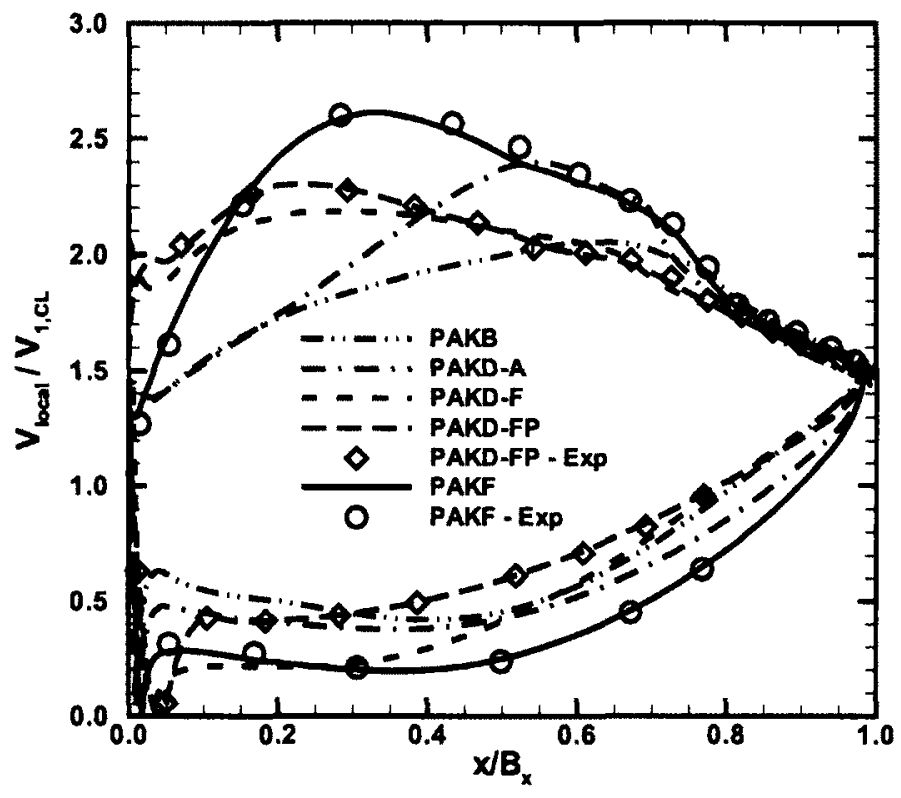

Figure 8.1: Computed loading distribution with experimental validation for five planar cascades, $R e=1.26 \times 10^{5}$ 
When designing high-lift airfoils the strength of the adverse pressure gradient sustainable along the suction surface is of concern. The predicted velocity distribution along the suction surface is plotted relative to the fraction of suction surface length $\left(s^{*}\right)$ in Figure $8.2 \mathrm{a}$. The stagnation point is marked by $s^{*}=0$, while $s^{*}=1$ denotes the bisection of the trailing edge circle. It should be noted that the stagnation point lies on the geometric pressure surface of the airfoils at design incidence and that Figure 8.2 captures the acceleration of the flow around the leading edge. The parameter plotted on the ordinate axis is the ratio of local isentropic velocity relative to the exit midspan mass-averaged velocity. Figure $8.2 \mathrm{~b}$ plots the same isentropic velocity ratio relative to the absolute surface distance in $\mathrm{mm}$, where 0 marks the bisection of the trailing edge circle. The curves in Figure $8.2 \mathrm{~b}$ are representative of the pressure gradient seen by the boundary layer. In general the trends in Figure $8.2 \mathrm{a}$ match those of Figure 8.1 but the distributions appear more forward-loaded when plotted relative to $s^{*}$ rather than axial chord. The velocity distributions in both Figures $8.2 \mathrm{a}$ and $8.2 \mathrm{~b}$ collapse to a single slope over the aft $30 \%$ of suction surface length.

a)

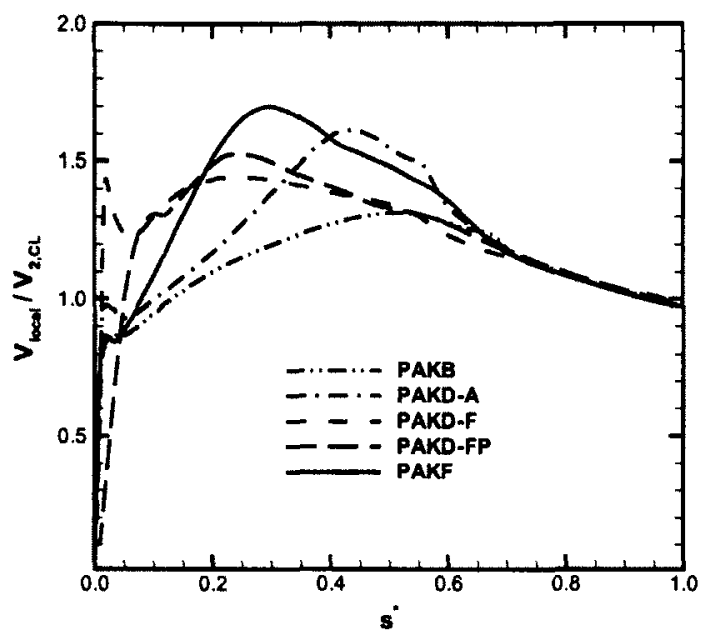

b)

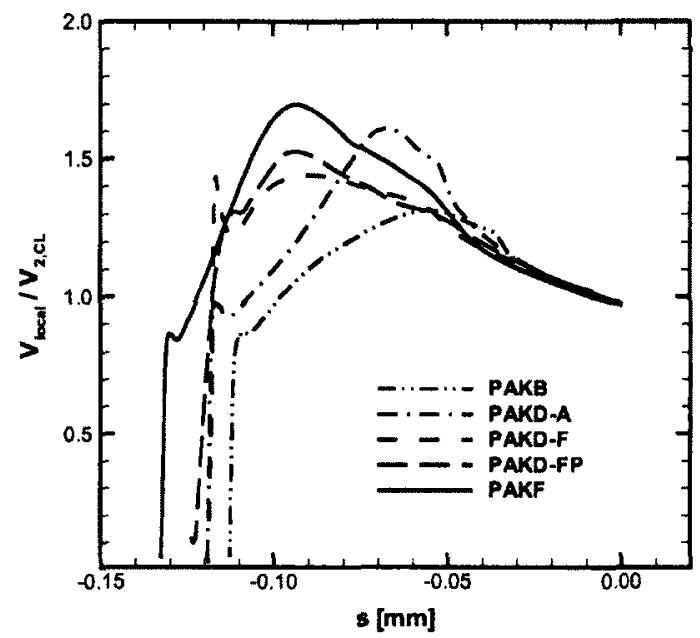

Figure 8.2: Velocity ratio along suction surface, $R e=1.26 \times 10^{5}$

Table 8.1 summarizes the isentropic velocity on the suction surfaces of the various 
cascades. The ratio of maximum velocity to exit velocity $\left(V_{\max } / V_{2}\right)$ is tabulated along with the location of the peak velocity as a fraction of suction surface length $\left(s_{\text {peak }}^{*}\right)$. Like $s^{*}, s_{\text {peak }}^{*}=0$ corresponds to the stagnation point and $s_{\text {peak }}^{*}=1$ marks the bisection of the trailing edge circle. The non-dimensional deceleration parameter $(D)$, created by the present author to evaluate the level of diffusion along the suction surface, is also tabulated. The denominator of the deceleration parameter normalizes the distance along the suction surface downstream of the location of peak velocity, by the axial chord. The denominator also accounts for the fact that the magnitude of suction surface length $\left(S_{\max }\right)$ varies across the airfoils tested. A larger value of deceleration parameter is indicative of a stronger adverse pressure gradient overcome by the suction surface boundary layer.

Table 8.1: Summary of suction surface peak velocity for PAKB, PAKD-A, PAKD-F, PAKDFP and PAKF

\begin{tabular}{lccc} 
Blade & $V_{\text {max }} / V_{2}$ & $s_{\text {peak }}^{*}$ & $D=\frac{V_{\text {max }} / V_{2}}{\left(\frac{\left(1-s_{p e a k}^{*}\right) \cdot S_{\text {max }}}{B_{x}}\right)}$ \\
& & & 1.79 \\
\hline PAKB & 1.32 & 0.51 & 1.82 \\
PAKD-A & 1.61 & 0.44 & 1.20 \\
PAKD-F & 1.43 & 0.25 & 1.23 \\
PAKD-FP & 1.52 & 0.25 & 1.37 \\
PAKF & 1.69 & 0.29 & \\
\hline \hline
\end{tabular}

Although PAKF has the highest peak velocity, the aft loaded PAKD-A and PAKB cascades experience the strongest deceleration downstream of the suction peak. The weaker deceleration for PAKF is partly a result of the forward location of the suction peak, but also a product of the high camber generating a longer suction surface length $\left(S_{\max }\right)$. The deceleration parameter denotes an average value for the diffusion of the flow along the suction surface. A single average value is a reasonable representation for PAKB, PAKD-F and PAKD-FP since the diffusion downstream of the suction peak is more or less uniform. PAKD-A and PAKF on the other hand could have the diffusion characterized by two values, 
one from $s_{\text {peak }}^{*}<s^{*}<0.7$ and a second from $0.7<s^{*}<1.0$ (Figure 8.2a).

A turbine blade row is required to turn and accelerate the flow. The exit flow angle of a stator row is critical to the performance of the downstream rotor. Ideally the exit flow angle would be constant with Reynolds number so that the design point velocity triangles would be unaffected by altitude. The exit flow angle is plotted relative to Reynolds number (based on inlet velocity and axial chord) for the various cascades in Figure 8.3. The PAKF results were taken by the present author while the data for the other blade rows was measured by Popović (2005). PAKB has a relatively uniform exit flow angle at high Reynolds number. The exit flow angle decreases (the blade does less turning) as the Reynolds number is reduced and the suction surface separation bubble grows. PAKD-F on the other hand shows a slight increase in flow turning as the Reynolds number is reduced which is believed to be a result of an increased axial velocity ratio (Popović, 2005). PAKD-A exhibits a marked reduction in flow turning with lower Reynolds number eventually leading to stall at $3 \times 10^{4}$ (test point not shown).

Like PAKB and PAKD-A, PAKF shows some reduction in flow turning with a reduction in Reynolds number. The mixed-out midspan flow angle is reduced by about 1.5 degrees at a Reynolds number of $2.5 \times 10^{4}$ relative to the design point condition of $8 \times 10^{4}$. This reduced flow turning is greater than that seen from PAKB, yet less than what is produced by PAKD-A which is stalled at the lowest operating point measured. PAKF also generates less flow turning as the Reynolds number is increased above the design point. At the highest Reynolds number tested $\left(1.35 \times 10^{5}\right)$ the flow turning is reduced by about 0.7 degrees relative to the design condition. It should be noted that the trend in flow angle discussed here is approaching the experimental uncertainty for the three-hole probe quoted in Section 3.9.2; however, a large component of the absolute measurement uncertainty is the repeatability to which the probe can be mounted in the calibration box or the test section. Since these measurements were taken sequentially with the probe installed in the test section a single 


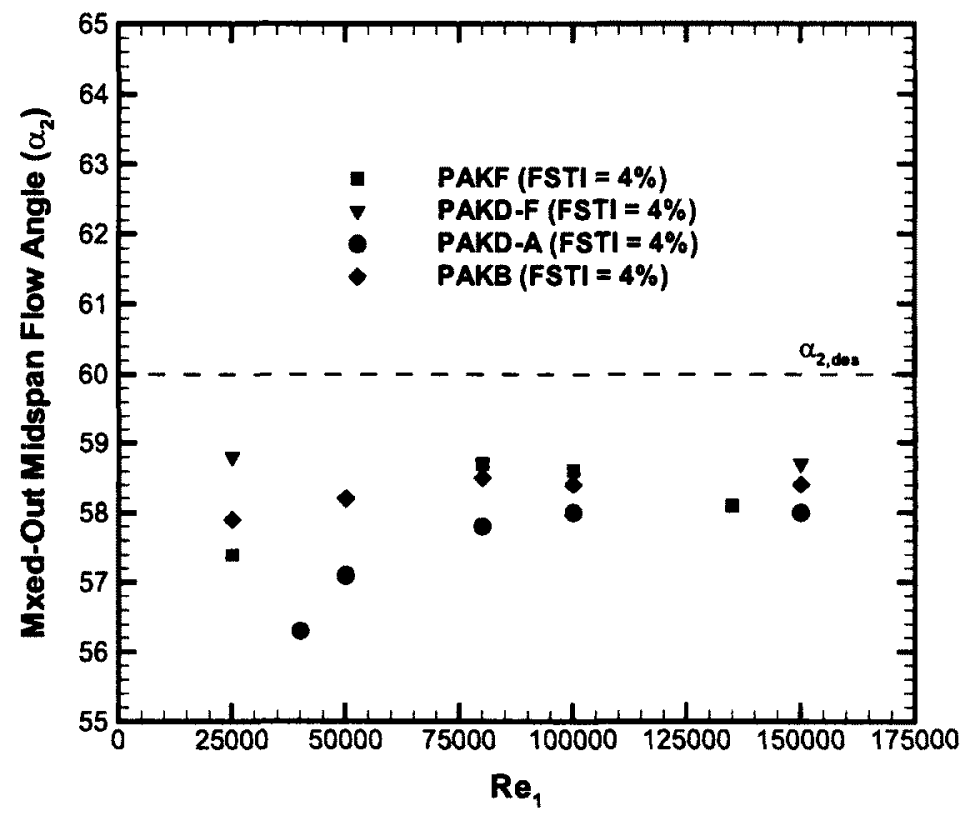

Figure 8.3: Mixed-out midspan flow angle vs. Reynolds number

time, this bias error can be ignored.

The reduction in flow angle for PAKF at high Reynolds number may be a result of the onset of high Reynolds number stall, where the suction surface boundary layer undergoes a turbulent separation near the trailing edge and fails to reattach (See Hourmouziadis (1989) Figure 23). Evidence of high Reynolds number stall could been seen by examining the total pressure deficit in the wake as well as the wake location relative to the trailing edge.

The measured total pressure deficit coefficient is plotted relative to non-dimensional pitchwise location for PAKF at the various Reynolds numbers tested in Figure 8.4. The location of the trailing edge projection is also marked. A negative pitchwise coordinate corresponds to the pressure side of the blade passage. The momentum deficit in the wake is a result of the viscous dissipation in the pressure and suction surface boundary layers, as well as the sudden expansion occurring at the trailing edge. The largest total pressure 
deficit and widest wake occurs at the lowest Reynolds number. The wakes of the two subsequently higher Reynolds numbers tested appear quite similar to one another with the wake at $8 \times 10^{4}$ being slightly wider and having a larger maximum pressure deficit than that of $1 \times 10^{5}$. The pressure side of the wake, nearest to $y / s=0$, is nearly coincident for the three lowest Reynolds numbers tested. This suggests that the variation in profile loss generated by PAKF is dominated by changes to the suction surface boundary layer.

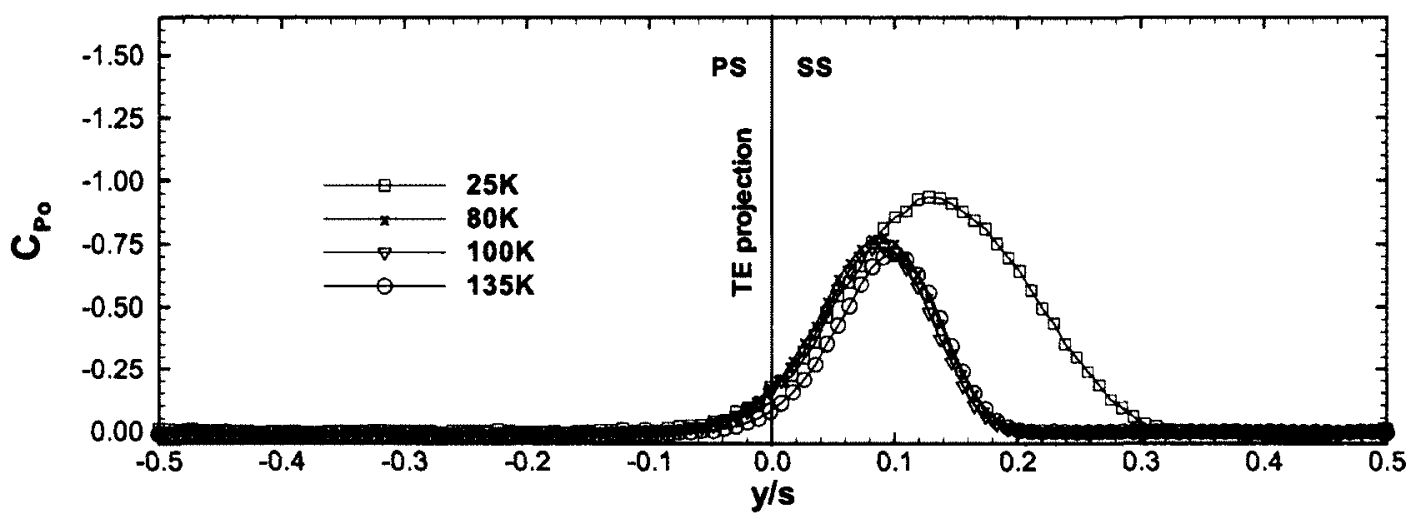

Figure 8.4: Characteristics of wakes with Reynolds number variation for PAKF at $140 \%$ axial cord

At $R e=1.35 \times 10^{5}$ however, the pressure side of the wake is shifted away into the suction side of the passage being driven by the axial momentum of the fluid. Since the pressure deficit and wake width continue to decrease it is unlikely that an unattached turbulent separation exists near the trailing edge at this operating point. However, it is plausible that increasing the axial momentum of the fluid with Reynolds number and the thickening of the suction surface boundary layer would eventually lead to such a separation. The deflection of the wake toward the suction side of the passage is in accord with the midspan flow angle data presented above which shows a reduced flow turning at high Reynolds number.

Figure 8.5 shows the mixed-out midspan losses for PAKF measured by the present author over the range of inlet Reynolds numbers. The results are compared to those of PAKB 
measured by Popović (2005). The profile losses of PAKF are similar to those of PAKB for the range of Reynolds number tested. This is truly remarkable as PAKF has $65 \%$ higher blade loading than PAKB, as determined by the Zweifel coefficient. Also notable is that the flow is well behaved at low Reynolds number for PAKF. In fact, the PAKF hardware was designed with both active and passive flow control in anticipation that the airfoil may show severe separation or stall, even at the design condition.

The fact that PAKB and PAKF have similar profile losses is somewhat surprising. As noted previously, the entropy generation in an attached boundary layer scales on the boundary layer edge velocity to the third power (Denton, 1993). Consequently the loss generation is typically said to be dominated by the suction surface boundary layer. Examining Figure 8.2, PAKF has higher velocity along the suction surface than PAKB over almost $70 \%$ of the suction surface length. With both airfoils having nearly the same suction surface length, the profile losses for PAKF would be thought to be higher than those of PAKB. However, the loss generated in the attached suction surface boundary layer is only part of the overall loss measured downstream of the blade row.

Along the pressure surface, PAKF has a lower local velocity than PAKB (Figure 8.1). Over the first $60 \%$ of axial chord PAKF has a local velocity nearly half that of PAKB. In addition, Mahallati (2003) found the presence of a pressure surface separation for PAKB for an inlet Reynolds number less than 150,000. Given this, the loss generation in the pressure surface boundary layer for PAKF may be less than that of PAKB, assuming a similar dissipation coefficient.

The presence of a suction surface separation bubble also contributes to the entropy generation in the boundary layer. Figure 5.15 showed that PAKB has a midspan separation bubble that spans approximately $10 \%$ of $s^{*}$ at a Reynolds number of $1.26 \times 10^{5}$. Although the oil surface flow visualization for PAKF is not shown, it indicates the presence of a small 


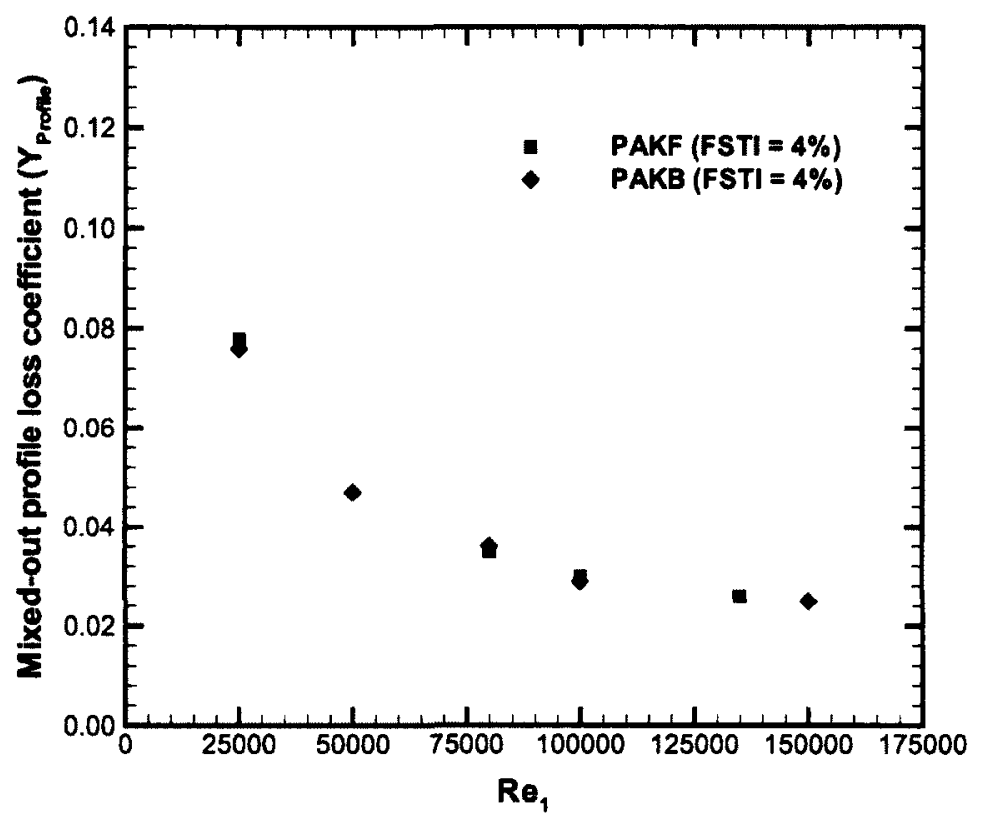

Figure 8.5: Mixed-out profile loss vs. Reynolds number

midspan separation bubble that spans roughly $3 \%$ of $s^{*}$. The smaller separation bubble should generate less loss.

It is noteworthy that the CFD predictions for PAKB (Figure 5.15) appear to over predict the rate of transition in the free shear layer which results in a shorter separation bubble. The midspan simulations for PAKF presented in Section 4.3.4 do not predict the presence of a separation bubble as the boundary layer undergoes transition to turbulence under attached conditions upstream of the separation location. These results further support the findings of the previous chapter concerning the difficulty in capturing the behaviour of the suction surface boundary layer with a transition model.

Little loss data exists in the open literature for extremely highly loaded linear turbine cascades, but a hesitant comparison can be made with the results of Houtermans et al. (2004). The profile losses for PAKF are lower than those generated by the Houtermans 
et al. (2004) cascade, which has a Zweifel coefficient of 1.473 whereas $Z_{w}=1.78$ for PAKF. At a Reynolds number of $1.35 \times 10^{5}$ PAKF generates roughly $30 \%$ less midspan total pressure loss, while at $R_{e}=2.5 \times 10^{4}$ the profile loss for PAKF is about $70 \%$ lower. The comparison of PAKF with the Houtermans et al. (2004) cascade is made hesitantly since their results were taken with an inlet Free Stream Turbulence Intensity (FSTI) of $0.6 \%$ while the PAKF results were taken with an FSTI of 4\%. Data from Popović (2005) and Mahallati (2003) show slight improvements in profile loss with increased FSTI for attached boundary layers having a separation bubble. Thus, if the Houtermans et al. cascade were tested at a higher FSTI the performance gap could be somewhat smaller. On the other hand, it is plausible that the suction surface boundary layer of PAKF would have a larger separation bubble and thus give higher losses if it were tested at a FSTI of $0.6 \%$.

Thus far it has been shown that it is possible to design an extremely highly loaded lowpressure turbine cascade that has good midspan performance in comparison to others in the same family or to those found in open literature. The following subsection will examine the corresponding secondary flows and endwall losses generated.

\subsubsection{Secondary Flow}

The secondary flow structures for PAKF, measured at $140 \%$ axial cord downstream of the leading edge, are compared to those of PAKB, PAKD-A and PAKD-F in Figure 8.6. The figure shows flood contours of streamwise vorticity coefficient $\left(C_{\omega s}\right)$ overlaid with line contours of total pressure deficit coefficient $\left(C_{P_{o}}\right)$. The trailing edge projections are shown as dashed lines at $y / s=0$ and $y / s=1$. The passage vortex rotates clockwise and for the right-hand coordinate system selected, has negative streamwise vorticity. The passage vortex for PAKF is the strongest of the airfoils tested and is roughly $10 \%$ more intense than that of PAKD-F as measured by the streamwise vorticity. The counter vortex is located 
furthest away from the endwall (about $18 \%$ blade span away from the wall) and is comprised of fluid having positive streamwise vorticity. This anticlockwise rotating vortex has peak intensity that is similar across the four cascades, with PAKD-F having a counter vortex that is about $5 \%$ more intense than that of PAKF. Near the endwall, is a second vortex comprised of fluid having positive streamwise vorticity. This is the corner vortex that forms within the blade passage along the junction of the suction surface of the blade with the endwall.

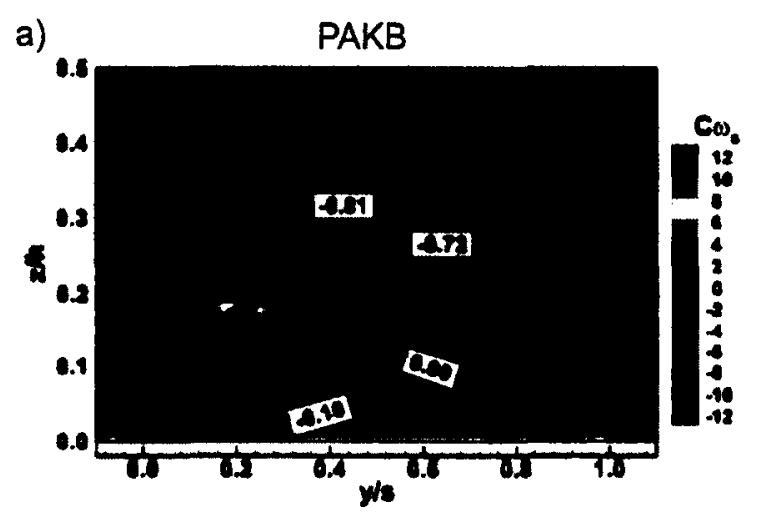

b)

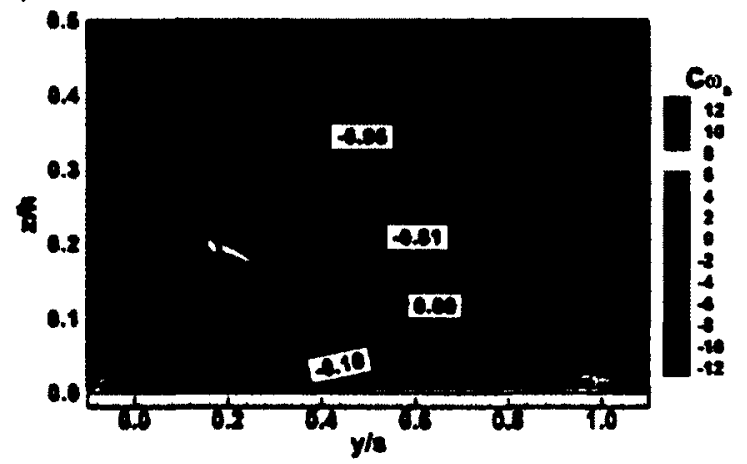

c)

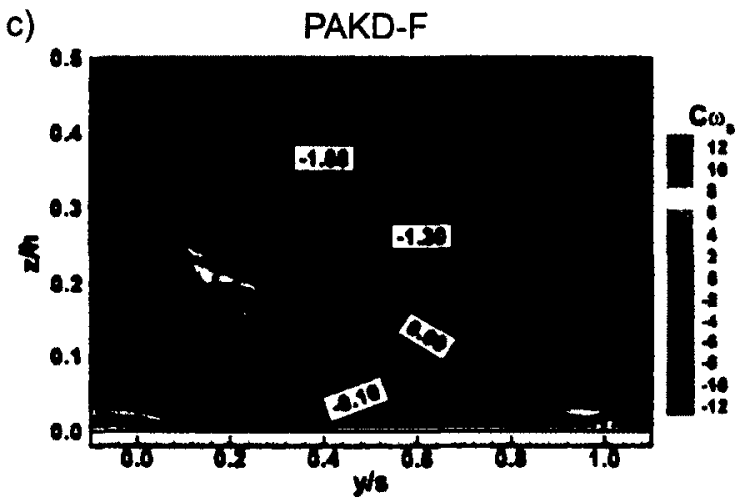

d)

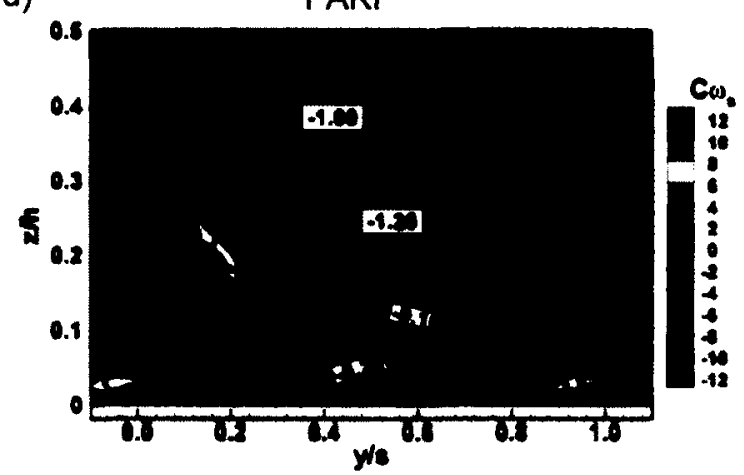

Figure 8.6: Line contours of $C_{P_{0}}$ with flood of $C_{\omega s}$, at $140 \%$ axial cord for PAKB, PAKD-A, PAKD-F and PAKF

The line contours of Figure 8.6 have the peak level of total pressure deficit coefficient $\left(C_{P o}\right)$ marked. The loss core furthest from the endwall is thought to be comprised of suction surface boundary layer fluid that is displaced by the endwall flow. For PAKF this region has peak $C_{P o}=-1.0$. This is the same as the peak level of loss for this core generated 
by PAKD-F and is greater than that produced by the other cascades. In the most highly loaded case (Figure 8.6d), this high loss fluid has migrated further from the endwall in comparison to the other test cases, being displaced by the secondary flow beneath it. Also in Figure 8.6d, the loss core associated with the passage vortex is marked by the $C_{P_{o}}=-1.2$ arrow. This level of passage vortex total pressure deficit is second only to that of PAKD-F. In between the two loss cores indicated by the arrows is a region containing lossy fluid. In previous chapters it was shown that this lossy fluid originates as higher energy fluid that was driven across the passage, near the endwall underneath the passage vortex and then enveloped around it. In Figure 8.6d, the two loss cores indicated by the arrows are separated by the furthest distance when compared to the other blade rows. This suggests that more fluid is driven across the passage and into the secondary loss structure.

\subsubsection{Summary of Results}

Selected measured and mixed-out exit plane results for all the cascades tested are tabulated in Table 8.2. PAKF has the strongest secondary flow as determined by the coefficient of secondary kinetic energy $\left(C_{S K E}^{\prime \prime}\right)$. Pervious chapters showed a link between stronger secondary kinetic energy and more high energy fluid being drawn into the secondary flow structure as described above. This exacerbates loss generation in the endwall flow up to the measurement plane by increased mixing. In addition, when performing the calculation to the hypothetical the mixed-out plane, the secondary kinetic energy is summed with the diffusion loss, to compute the mixed-out loss. So PAKF suffers a greater penalty for generating the strongest endwall flow.

Although the various cascades generate similar profile losses $\left(Y_{\text {profile }}\right)$, they exhibit a significant difference in overall row loss $\left(Y_{\text {total }}\right)$. PAKF, having the worst performance, has a row loss that is over $80 \%$ higher than that of the baseline airfoil, PAKB. The poor 
Table 8.2: Summary of experimental planar endwall results for PAKB, PAKD-A, PAKD-F, PAKD-FP and PAKF

\begin{tabular}{lcrrrrr}
\hline \hline LOCATION & QUANTITY & PAKB & PAKD-A & PAKD-F & PAKD-FP & PAKF \\
\hline OUTLET & $Z_{w}$ & 1.08 & 1.36 & 1.36 & 1.36 & 1.78 \\
$B_{x}=1.40$ & $C_{S K E}^{\prime \prime}$ & 0.015 & 0.021 & 0.031 & 0.017 & 0.041 \\
\hline MIXED-OUT & & & & & & \\
& $Y_{\text {total }}$ & 0.052 & 0.072 & 0.082 & 0.059 & 0.095 \\
& $Y_{\text {profile }}$ & 0.025 & 0.032 & 0.032 & 0.031 & 0.029 \\
& $Y_{\text {secondary }}$ & 0.027 & 0.040 & 0.050 & 0.028 & 0.066 \\
& $\alpha_{2}^{\prime \prime}[\mathrm{deg}]$ & -57.7 & -56.8 & -57.7 & -57.5 & -57.5 \\
\hline \hline
\end{tabular}

performance for PAKF is primarily a result of the secondary losses generated, which are almost one and a half times higher than those of PAKB.

\subsubsection{The Use of High-Lift Airfoils to Expand the Design Envelope}

It is unlikely that extremely highly loaded blading would be attractive in the design of a civil flight engine. These engines are typically constrained to have optimum thermodynamic efficiency at the cruise condition (although this may not always be the case depending on the characteristic mission of the aircraft). There is a tradeoff between engine weight and the required aircraft lift and drag required to carry the powerplants and fuel, but a designer would not accept a significant penalty to the isentropic efficiency of the low-pressure turbine.

PAKF was not designed as a candidate for engine application. Rather, it was intended to test the capabilities of the design tools under extreme conditions. It has demonstrated that current state of the art tools can be used to predict boundary layer transition to the accuracy required to design airfoils having very high Zweifel coefficients, with acceptable profile losses. The success of PAKF builds confidence in the design tools to expand the design space in a much more moderate way.

PAKF generates strong endwall flows and high secondary loss. Even with the implementation of endwall contouring it is extremely unlikely that the endwall losses of PAKF 
could be brought to a level comparable to those of PAKB. Chapters 5 and 6 showed that the effectiveness of endwall contouring was improved at higher blade loading and that a $32 \%$ reduction in secondary loss was possible in the highly-loaded case (PAKD-F). Even with a scaled reduction in secondary loss, PAKF would still have higher row loss than the baseline airfoil PAKB. In Chapter 7 it was demonstrated that an almost $40 \%$ reduction in secondary loss was achievable with the modified loading approach. In Figure 8.1 it can been seen that PAKF has lower streamwise momentum along the pressure surface than PAKDF. Thus PAKF would be a candidate for the modified loading approach. When applying the modified loading approach the velocity on the suction surface must also be increased to maintain the same overall loading level; however, the peak suction surface velocity for PAKF is already high (Figure 8.1), leaving little room to apply the modified loading approach without incurring further losses generated by shocks in an engine environment. Wisler (1998) estimated that for modern engines a $1 \%$ change in low-pressure turbine efficiency corresponds to a $1 \%$ change in specific fuel consumption. Thus even a small turbine efficiency penalty is consequential. Based on these considerations, it is highly unlikely that a blade loading level comparable to PAKF would be attractive for a commercial flight power plant.

The data set generated for this thesis can be used to make some comments as to a loading level that could be successfully employed in a high-lift low-pressure turbine design, taking the PAKB planar endwall losses as the benchmark. Admittedly these results are from an idealized approximation to a real low-pressure turbine and are specific to a family of airfoils having the same velocity triangles.

The mixed-out secondary loss $\left(Y_{\text {sec }}\right)$ computed from the $140 \% B_{x}$ plane measurements for all the test cases examined thus far are presented in Figure 8.7. Forward loading has been shown to be attractive from the perspective of midspan performance of high-lift designs, and therefore the planar endwall results for PAKF and PAKD-F are connected to that of 
PAKB by a curve. There is a trend of increasing secondary loss with increased Zweifel coefficient, but the loading distribution clearly also has a significant impact, as can be seen at the elevated loading level of the PAKD airfoils. At a Zweifel coefficient of 1.36, PAKD-A and PAKD-FP generate lower losses than PAKD-F, with PAKD-FP producing nearly the same $Y_{\text {sec }}$ as PAKB.

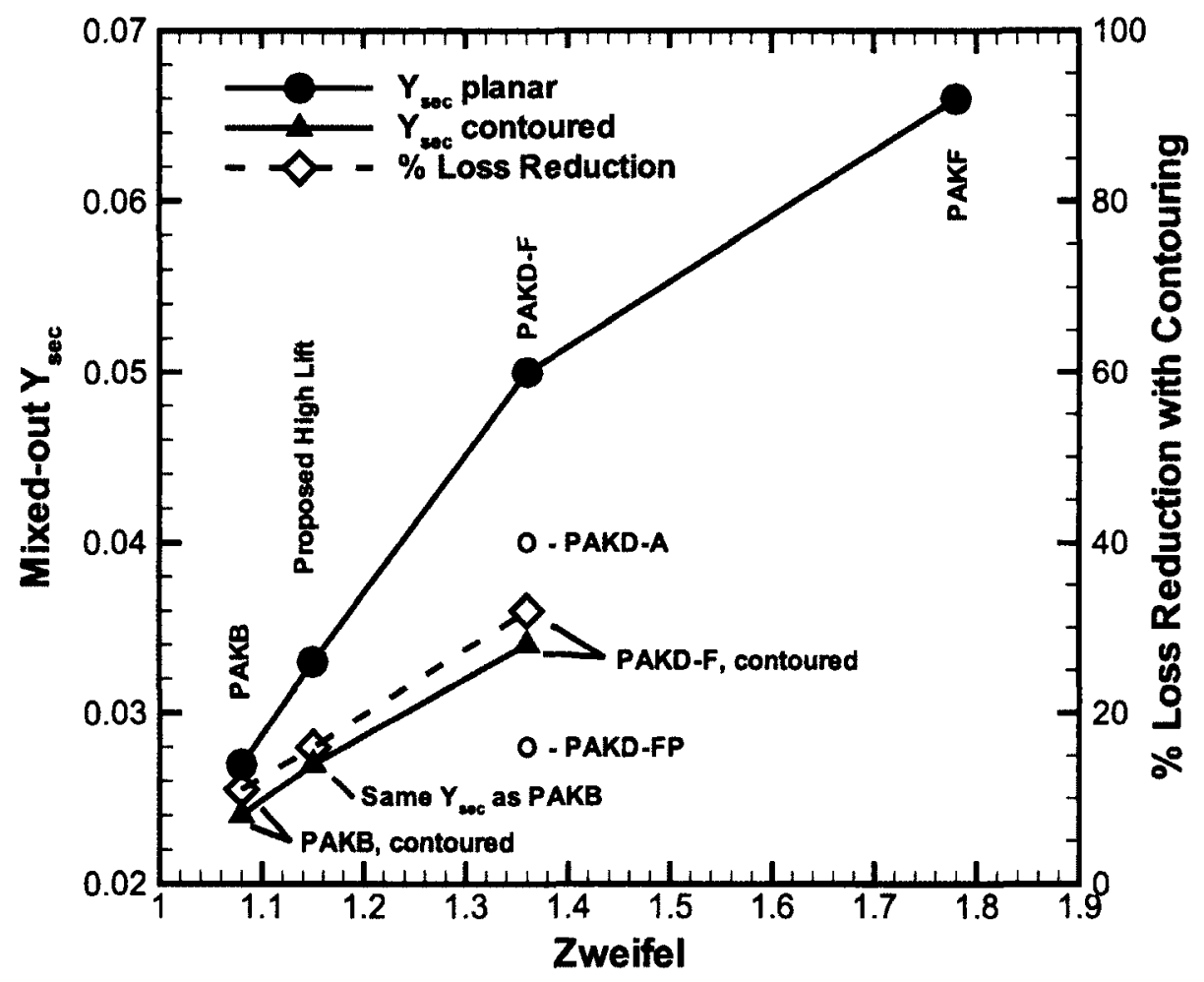

Figure 8.7: Secondary loss vs. Zweifel coefficient and the use of high-lift airfoils

In Chapter 5 it was shown that secondary loss can be mitigated with the use of nonaxisymmetric endwall contouring. Chapter 6 demonstrated that the contouring was even more effective when applied to a forward-loaded, high-lift design. The mixed out secondary losses for the two contoured cases are also plotted on Figure 8.7 and connected by a curve. The second ordinate axis plots the percent reduction of secondary loss resulting from the 
application of endwall contouring for the test case.

Examination of the results of the previous chapters suggests that at $25 \%$ reduction in blade count, that is substituting PAKD generation of airfoils for PAKB may be too ambitious. Although the modified loading approach (PAKD-FP) produced nearly the same secondary loss as PAKB, it generated higher profile loss and subsequently higher row loss. The application of endwall contouring to PAKD-F was unable to bring the row loss to the reference level. However, taking PAKB, with a planar endwall as the reference, it should be possible to design a replacement stage with a higher Zweifel coefficient without incurring higher row loss by implementing state of the art three-dimensional design techniques. Applying the admittedly crude assumptions that the secondary losses and loss reduction potential of endwall contouring scale linearly yields a Zweifel coefficient of about $Z_{w}=1.15$ as a plausible high-lift replacement for PAKB. A caveat to this is that PAKB does not necessarily represent the best efficiency possible at a $Z_{w}=1.08$ and a careful redesign could avoid pressure and suction surface separation bubbles. This would thereby reduce the overall row loss and provide a new reference efficiency. One could then apply endwall contouring to this new design to further improve the performance.

To this author's knowledge, only a single study exists in the open literature discussing the optimum level of lift for a low-pressure turbine design, although it is likely that each gas turbine manufacturer would attempt a similar exercise examining high-lift blades. Gier et al. (2010) examined the results of three real speed full scale turbine rigs in an altitude test. They incorporated high-lift designs to various stages throughout the machines and documented the change in efficiency. The authors summarize their results by recommending a Zweifel coefficient in the range of $0.8-1.0$. The authors note that their results are limited to annular endwalls and that three-dimensional optimization of the endwall should move the optimal Zweifel to higher levels. Thus, the recommendations of Gier et al. (2010) appear to be in relatively good agreement with the findings of the present work, despite the current 
data being from a simplified, low speed representation of a stator vane row. 


\section{Chapter 9}

\section{Summary, Contributions,}

\section{Conclusions and Recommendations}

\subsection{Summary}

In a modern high bypass ratio turbofan engine the low-pressure turbine can account for as much as one third of the total weight of the machine. The weight of the low-pressure turbine can be decreased by using fewer blades in the rotating and non-rotating components. This has the added benefit of lower material and manufacturing costs. By reducing the blade count, each airfoil must exert a stronger aerodynamic force for the same stage loading. Traditionally, it is accepted that high-lift airfoils are less efficient, generating higher profile and secondary losses than conventional-lift blades. It is estimated that a $1 \%$ drop in lowpressure turbine efficiency corresponds to a $1 \%$ increase in specific fuel consumption. Thus, even a small efficiency penalty is consequential to the overall performance of the engine.

Previous to this work, it was found that forward loading could be used to design highlift airfoils having good midspan performance. Forward loading could be used to initiate 
the transition to a turbulent boundary layer thereby avoiding the presence of a separation bubble on the suction surface. As a result, the efficiency of forward-loaded high-lift designs was relatively insensitive to Reynolds number. However, forward-loaded blades have been shown to generate strong endwall flows and high secondary loss. It has been the goal of this thesis to demonstrate the feasibility of employing high-lift airfoils with the use of secondary loss reduction strategies. Two secondary loss reduction strategies were investigated. The first, non-axisymmetric endwall contouring involves a three-dimensional optimization of the endwall geometry. The optimization is specific to the loading distribution of the airfoil and requires an iterative numerical solution of the flow field through the blade passage. The second method entails a alteration of the two-dimensional cross-section of the blade. The intent of this modified-loading approach is to reduce the region of near-stagnation of the fluid where the pressure face of the blade meets the endwall. The near-stagnation region of the fluid was reduced by increasing the streamwise momentum. Consequently, the pressure on the suction-side of the passage must also be reduced to maintain the original loading level.

Both secondary loss reduction strategies were assessed in a low-speed linear cascade test facility. A low-speed linear cascade provides a simplified, controlled and cost effective environment for evaluating technologies that are low on the "technology readiness level" scale. Detailed pressure probe measurements were taken upstream of the blade row, throughout the blade passage, and downstream of the trailing edge to document the threedimensional flow field and loss generation. Oil surface flow visualizations of the blade suction surface and endwall were used to assist in the interpretation of the pressure-probe results. The PAK family of low-pressure turbine airfoils were used for this work. These airfoils and secondary loss reduction strategies were designed by Pratt and Whitney Aircraft (PWA) using proprietary design tools. The PAK family of airfoils have the same inlet and outlet velocity triangles and have a systematic variation in blade loading and loading distribution 
which make them useful for studying the endwall flows.

The baseline airfoil in the family, designated PAKB has a Zweifel loading coefficient of 1.08 and is representative of the midspan cross section of a PWA low-pressure turbine blade. The subsequent generation of the family, PAKD-F and PAKD-A have $25 \%$ higher loading than the baseline case and employ forward and aft loading distributions, respectively. The forward-loaded airfoil (PAKD-F) was redesigned employing the modified-loading approach described above. This modified airfoil carries the designation PAKD-FP. A fifth extremelyhighly-loaded blade row, PAKF is also investigated. To the author's knowledge PAKF is the most highly loaded airfoil found in open literature, having a Zweifel loading coefficient of 1.78 .

\subsection{Contributions}

A number of contributions were made throughout this body of work. These contributions are outlined in this section and elaborated upon in the following one.

A new design approach for non-axisymmetric endwall contouring was validated experimentally. Where previous studies on endwall contouring focused on optimizing the design for a particular airfoil, the focus here was to apply contouring to high-lift designs. The contouring was found to be more effective when employed to higher lift airfoils and seems particularly useful for forward-loaded blades. It is proposed that endwall contouring and forward-loading could be used in conjunction to design a high-lift stator. Forward-loading provides good midspan performance and little sensitivity to Reynolds number. Moreover, the use of endwall contouring results in less secondary kinetic energy downstream of the blade row allowing for more work extraction in the subsequent rotor.

A novel design approach for blade loading was experimentally validated. The modified 
loading approach yields the benefits of endwall contouring without additional manufacturing complexity. In addition, it was shown that it is possible to design an ultra-high-lift forwardloaded airfoil with comparable midspan performance to the baseline. In broad terms it can be said that the data presented demonstrates the potential to expand the design envelop of a low-pressure turbine to employ higher-lift airfoils without significant sacrifice to performance. In the previous chapter it was reasoned that a Zweifel coefficient of 1.15 represents a sensible upper limit for design while maintaining entropy generation to current standards.

A primary focus of this work has been to develop a better understanding of the flow field around low-pressure turbine airfoils. A new miniature seven-hole pressure probe and mounting system were designed for the purpose of taking intra blade passage measurements. Although this has been done by other researchers, the measurements taken by the present author are of a resolution that is sufficient to resolve the entrainment of higher energy fluid into the endwall flow structure. The detailed measurements have allowed new insights into the flow physics and mechanisms of entropy generation to be proposed.

The oil surface flow visualizations were performed in addition to the pressure probe measurements. The visualizations were used to infer details regarding the penetration depth of the secondary flow for the range of airfoils tested. The trends identified are contrary to those commonly accepted. That is, higher or forward loading causes the secondary flow to penetrate further from the endwall and produce higher secondary loss. Concerns have been raised as to the ability of correlations in open literature to predict the penetration depth of the secondary flow for planar endwalls. This warrants attention as these correlations are used to predict efficiency and make decisions early in the design cycle. It has been shown that the penetration depth, and the secondary losses generated are inversely proportional to the extent of near-stagnation $\left(V_{\text {local }} / V_{1}\right)$ that occurs locally on the pressure surface of the blade. 
Computational Fluid Dynamics (CFD) simulations were carried out to supplement the experimental measurements. It has been shown that despite their limitations, state-ofthe-art computational methods are capable of predicting boundary layer transition to a level necessary for designing extremely highly-loaded blades. Also, modern computational methods can predict trends in endwall loss with slight perturbations in geometry as is required for the optimization of endwall contouring.

\subsection{Conclusions}

Non-axisymmetric endwall contouring was applied to the baseline PAKB cascade. This validated the PWA design methodology. Furthermore, it verified that the sensitivity of the experiment was sufficient to resolve subtle differences in performance and flow physics imposed by the contouring. It was found that the contouring was capable of reducing secondary losses by $15 \%$. Non-axisymmetric endwall contouring was subsequently applied to the higher-lift forward-loaded design, PAKD-F. It was found that a substantial secondary loss reduction of $32 \%$ was achievable.

It was found that the application of non-axisymmetric endwall contouring reduces the strength of the cross-passage flow near the endwall. This results in less high energy fluid being convected toward the suction-surface/endwall junction and eventually up the span of the blade. Subsequently there is less mixing in the secondary flow and lower production of entropy. It was also seen that the endwall contouring can divide the vorticity associated with the passage vortex into two distinct vortices within the blade passage. These two vortices have lower peak vorticity that results in less viscous dissipation at their cores. The pair of vortices also generate a weaker cross-passage flow near the endwall resulting in less convection of free-stream fluid into the secondary flow. In general it can be said that endwall contouring can reduce the secondary kinetic energy downstream of the blade 
thereby reducing entropy generation in subsequent stages of the machine.

The redesigned forward-loaded higher-lift airfoil employing the modified loading approach (PAKD-FP) was then investigated. The novel design technique produced a significant reduction in secondary loss (44\% relative to PAKD-F) and had row performance comparable to that PAKB with a planar endwall. The success of the modified loading approach also relied on a reduction in the strength of the secondary flow. Like endwall contouring, the modified loading produced a reduction in the strength of the cross-flow near the endwall and lower vorticity at the core of the passage vortex. The complementary CFD simulations were used to identify a mechanism for the reduction in passage vortex vorticity. Streamlines were traced from various locations in the inlet boundary layer. It was found that the modified loading approach promoted the streamlines deep within the viscous sublayer to interact with the boundary layer developing on the suction surface of the blade having opposing vorticity. Consequently the fluid from the viscous sublayer of the inlet boundary layer, initially having the strongest negative vorticity, does not feed into the passage vortex.

The final results chapter examines the flow field of the extremely-highly-loaded PAKF airfoil. PAKF has a Zweifel loading coefficient of 1.78 which is $65 \%$ higher than that of the baseline PAKB. The blade row is tested over a range of Reynolds numbers and is found to have midspan performance comparable to that of PAKB. In addition of the remarkable midspan performance, PAKF was found to produce over $80 \%$ higher row loss than PAKB as it generated the strongest endwall flows and subsequently, the highest secondary losses of all the cascades tested.

Computational Fluid Dynamics simulations were performed to complement the experimental measurements. The Reynolds-averaged Navier-Stokes (RANS) equations were solved by employing the Shear-Stress-Transport turbulence closure model. The transition 
of boundary layers developing on no-slip walls was simulated using the Gamma-Theta transition model. It was found that the CFD simulations assisted in the interpretation of the flow physics. A detailed grid independence study was presented and showed little difference in solution when element count was increased from 840,000 to $1,130,000$. It was also reasoned that a transition model was essential for predicting the flow through a lowpressure turbine cascade in order to avoid the overall losses being erroneously dominated by the profile loss.

Several strengths of the computational method were established throughout this work. It was seen that the RANS based solutions using the SST turbulence model coupled with the Gamma-Theta transition model can predict the flow through a low-speed linear cascade of low-pressure turbine blades with reasonable accuracy. The pitchwise exit flow angle was predicted with good accuracy. This is essential information for the design of the subsequent stage. The simulations were also capable of predicting changes in the flow resulting from the application of non-axisymmetric endwall contouring. Changes in performance with endwall contouring are predicted with trend accuracy.

Some limitations of the computational method were also identified. The RANS computations have difficulty predicting absolute level of total pressure loss as well as the diffusion and dissipation of flow structures. The use of a transition model for predicting the state of the suction surface boundary layer is also especially challenging. A set of boundary conditions that produced a reasonable prediction of the location of a laminar separation bubble and turbulent reattachment on one blade row did not fair well on the other cascades. In one test case the simulation showed a separation bubble where none existed in the experiment, while the opposite was seen in another. Furthermore, the prediction of blade row entropy generation was trend accurate for most, but not all cases. The same can be said for the absolute level of level of total pressure loss, which is over predicted by the simulations in most cases. Thus design decisions based on CFD results must be made with 
caution.

Experimentally it was seen that the secondary kinetic energy measured downstream of a blade row scales with the secondary loss generated. In the simulations the magnitude of the secondary kinetic energy downstream of the blade row is over predicted suggesting the secondary velocities generated within the blade passage are being dissipated too slowly by the turbulence model. However, the changes in secondary kinetic energy across all the test cases scales with loss with trend accuracy. Therefore the secondary kinetic energy can be used as the optimization criteria for the design of endwall contouring as suggested by some authors.

\subsection{Recommendations for Future Work}

A number of recommendations for follow up research can be made based on the findings presented.

The secondary losses generated by a blade row seem particularly sensitive to the loading distribution in the leading edge region. Also, the design of the non-axisymmetric endwall contouring is optimized for the pressure distribution at design incidence. Since changes in incidence particularly effect the pressure distribution in the forward part of the airfoil, a logical extension of the current test matrix would be to investigate the off design incidence performance of the endwall contouring.

It was shown that the effectiveness of non-axisymmetric endwall contouring increases with increasing blade loading and that it is particularly effective for forward-loaded airfoils. It would be of interest to optimize and test a contoured endwall design for the extremely high-lift airfoil PAKF to determine the amount of secondary loss reduction possible. Also, an optimized endwall could be designed for the airfoil employing the modified loading approach 
to determine if synergistic benefits exist.

A wealth of total pressure loss data for high-lift planar endwall cascades have been collected. These data include accompanying flow visualization results to measure the penetration depth of the secondary flow. It would be of value to revisit the secondary loss correlations in open literature to determine their applicability to high-lift airfoils. The correlations could perhaps be improved by implementing the observation that the penetration depth varies inversely as the level of near stagnation ( $\left.V_{\text {local }} / V_{1}\right)$ along the pressure face of the airfoil.

It was seen that current state of the art RANS computations have some difficulty predicting total pressure loss as well as the diffusion and dissipation of flow structures. This is believed to be a result of limitations with the modeling of turbulence and boundary layer transition. The current data set could be complemented with a number of turbulence measurements. Hotwire measurements could be taken normal to the blade suction surface of the various cascades at midspan. This would document the local boundary layer thickness as well as the level of freestream turbulence. It would also be advantageous to document the three dimensional flow field with time resolved measurements of the turbulence field within the blade passage and downstream of the cascade. This way the Reynolds stresses, turbulent kinetic energy, dissipation and local eddy viscosity could be evaluated and compared to the simulations.

The computations also had difficulty predicting transition in attached boundary layers for some of the highly-loaded airfoils. The prediction of transition location relies on an empirical correlation that is obtained from boundary layer experiments with streamwise pressure gradients. The airfoils used in this study have streamwise pressure gradients along the suction surface that are more severe than what is typically seen in the transition experiments. Therefore it is suggested that the transition experiments be revisited 
investigating the effects of stronger streamwise pressure gradients.

The linear cascade experiment used for this thesis is a highly simplified representation of the flow through an actual turbine. However a number of complexities could be introduced and studied in a cost effective, controlled environment. The roll up of the endwall flow and associated secondary losses are sensitive to the state of the boundary layer ahead of the blade row. The influence of the upstream cavity, the casing purge flow, or the skew of the inlet boundary layer could be investigated by modifying the test section. It should be noted that experiments investigating the effects of upstream cavity and casing purge flow are currently underway in both the low-speed and high-speed turbomachinery laboratories at Carleton University.

The low-speed linear cascade is a test environment used to qualify concepts that have a low technology readiness level. The effects of radial pressure gradient could be investigated in an annular rig and compressibility effects should be evaluated in a high-speed experiment. The secondary loss reduction strategy should then be proven in a scaled high-speed multistage turbine rig before being implemented in an engine design. 


\section{References}

Ainley, D. G. and Mathieson, G. C. R. (1951), "A Method of Performance Estimation for Axial Flow Turbines," $R$ \& $M$ 2974, British ARC.

ANSYS-CFX (2006), ANSYS-CFX, Release 11.0 Software Manual, 11.0 ed.

Aunapu, N. V., Volino, R. J., Flack, K. A. and Stoddard, R. M. (2000), "Secondary Flow Measurements in a Turbine Passage with Endwall Flow Modification," ASME J. Turbomach., 122, pp. 651-658.

Benner, M. W. (2003), "The Effects of Leading Edge Geometry on Profile and Secondary Losses in Turbine Cascades," Ph.D. thesis, Carleton University, Ottawa, Canada.

Benner, M. W., Sjolander, S. A. and Moustapha, S. H. (2004a), "Measurements of Secondary Flows Downstream of a Turbine Cascade at Off-Design Incidence," ASME paper No. GT-2004-53786, Vienna, Austria.

Benner, M. W., Sjolander, S. A. and Moustapha, S. H. (2004b), "The Influence of Leading Edge Geometry on Secondary Losses in a Turbine Cascades at the Design Incidence," ASME J. Turbomach., 126, pp. 277-287.

Benner, M. W., Sjolander, S. A. and Moustapha, S. H. (2005a), "An Empirical Prediction Method for Secondary Losses in Turbines: Part I - A New Loss Breakdown Scheme and Penetration Depth Correlation," ASME paper No. GT-2005-68637, Reno, USA. 
Benner, M. W., Sjolander, S. A. and Moustapha, S. H. (2005b), "An Empirical Prediction Method for Secondary Losses in Turbines: Part II - A New Secondary Loss Correlation," ASME paper No. GT-2005-68639, Reno, USA.

Benner, M. W., Sjolander, S. A. and Moustapha, S. H. (2006), "An Empirical Prediction Method for Secondary Losses in TurbinesPart I: A New Loss Breakdown Scheme and Penetration Depth Correlation," ASME J. Turbomach., 128, pp. 273-280.

Brear, M. J., Hodson, H. P., Gonzalez, P. and Harvey, N. W. (2002b), "Pressure Surface Separations in Low-Pressure Turbines-Part 2: Interactions With the Secondary Flow," ASME J. Turbomach., 124, pp. 402-409.

Brear, M. J., Hodson, H. P. and Harvey, N. W. (2002a), "Pressure Surface Separations in Low-Pressure Turbines-Part 1: Midspan Behavior," ASME J. Turbomach., 124, pp. 393-401.

Brennan, G., Harvey, N. W., Rose, M. G., Fomison, N. and Taylor, M. D. (2003), "Improving the Efficiency of the Trent 500-HP Turbine Using Nonaxisymmetric End Walls-Part I: Turbine Design," ASME J. Turbomach., 125, pp. 497-504.

Burd, S. W. and Simon, T. W. (2000), "Flow Measurements in a Nozzle Guide Vane Passage With a Low Aspect Ratio and Endwall Contouring," ASME J. Turbomach., 122, pp. 659-666.

Chen, L. D. and Dixon, S. L. (1986), "Growth of Secondary Losses Downstream of a Turbine Cascade," ASME J. Turbomach., 108, pp. 270-275.

Chung, J. T. and Simon, T. W. (1991), "Three-Dimensional Flow near the Blade/Endwall Junction of a Gas Turbine: Application of a Boundary Layer Fence," ASME paper No. 91-GT-45, Orlando, USA. 
Chung, J. T. and Simon, T. W. (1993), "Effectiveness of Gas Turbine Endwall Fences in Secondary Control at Elevated Freestream Turbulence Intensity," ASME paper No. 93-GT-51, Cincinnati, USA.

Cleak, J. G. E. and Gregory-Smith, D. G. (1992), "Turbulence Modeling for Secondary Flow Prediction in a Turbine Cascade," ASME J. Turbomach., 114, pp. 590-598.

Curtis, E. M., Hodson, H. P., Banieghbal, M. R., Denton, J. D., Howell, R. J. and Harvey, N. W. (1997), "Developement of Blade Profiles for Low-Pressure Turbine Applications," ASME J. Turbomach., 119, pp. 531-538.

de la Rosa Blanco, E., Hodson, H. P. and Torres, R. V. D. (2003), "Influence of the State of the Inlet Endwall Boundary Layer on the Interaction Between the Pressure Surface Separation and the Endwall Flows," Proceedings of the Institution of Mechanical Engineers, 217, pp. 433-441.

Deich, M., Zaryankin, A., Filippov, G. and Zatsepin, N. (1960), "Method of Increasing the Efficiency of Turbine Stages with Short Blades," Translation No. 2816, Associated Electrical Industries (Manchester) Limited.

Denton, J. D. (1993), "Loss Mechanisims in Turbomachines," ASME J. Turbomach., 115, pp. 621-650.

Dossena, V., D'Ippolito, G. and Pesatori, E. (2004), "Stagger Angle and Pitch-Chord Ratio Effects on Secondary Flows Downstream of a Turbine Cascade at Several Off-Design Incidences," ASME paper No. GT-2004-54083, Reno, USA.

Dossena, V., Perdichizzi, A. and Savini, M. (1999), "The Influence of Endwall Contouring on the Performance of a Turbine Nozzle Guide Vane," ASME J. Turbomach., 121, pp. 200-208. 
Dunham, J. and Came, J. M. (1970), "Improvements to the Ainley-Mathieson Method of Turbine Performance Prediction," ASME J. Eng. Power, 92, pp. 252-256.

Eckerle, W. A. and Awad, J. K. (1991), "Effect of Freestream Velocity on the ThreeDimensional Separated Flow Region in Front of a Cylinder," ASME J. Fluids Eng., 113, pp. 37-44.

Ellison, T. A. (2005), "Investigation of a Concept for Highly-Loaded Axial-Compressor Stators Using Flow Control," Final Report - Research Agreement No. 983061, Compressor Aerodynamics, Pratt $\&$ Whitney Canada, Ottawa, Canada.

Gallington, R. W. (1980), "Measurement of Very Large Flow Angles with Non-Nulling Seven-Hole Probes," USAFA paper No. TR-80-17.

Gerner, A. A. and Maurer, C. L. (1980), "Calibration of Seven-Hole Probes Suitable for High Angles in Subsonic Compressible Flows," USAFA paper No. TR-81-4.

Gerner, A. A., Maurer, M. L. and Gallington, R. W. (1984), "Non-Nulling Seven-Hole Probes for High Angle Flow Measurements," Experiments in Fluids, 2, pp. 95-103.

Gier, J., Franke, M., Hübner, N. and Schröder, T. (2010), "Designing Low Pressure Turbines for Optimized Airfoil Lift," ASME J. Turbomach., 132, pp. 12.

Goobie, S. M. (1989), "A Test Section for Investigating the Off-Design Performance of Planar Cascades," Master's thesis, Carleton University, Ottawa, Canada.

Gregory-Smith, D. G. (1997), "Physics of Secondary Flows," Secondary and Tip-Clearance Flows in Axial Turbines, edited by C. H. Sieverding, VKI Lecture Series 1997-01, Von Karman Institute for Fluid Dynamics, Rhode St. Genese, Belgium.

Gregory-Smith, D. G., Bagshaw, D., Ingram, G. L. and Stokes, M. (2008), "Using Profiled Endwalls, Blade Lean and Leading Edge Extensions To Minimise Secondary Flow," ASME paper No. GT-2008-50811, Berlin, Germany. 
Gregory-Smith, D. G. and Cleak, J. G. E. (1992), "Secondary Flow Measurements in a Turbine Cascade With High Inlet Turbulence," ASME J. Turbomach., 114, pp. 173183.

Gregory-Smith, D. G. and Graves, C. P. (1983), "Secondary Flows and Losses in a Turbine Cascade," Viscous Effects in Turbomachines, number 17 in 'AGARD-CP-351', Advisory Group for Aeronautical Research and Development, pp. 1-24.

Gregory-Smith, D. G., Graves, C. P. and Walsh, J. A. (1988), "Growth of Secondary Losses and and Vorticity in an Axial Turbine Cascade," ASME J. Turbomach., 110, pp. 1-8.

Hartland, J., Gregory-Smith, D. G., Harvey, N. W. and Rose, M. G. (2000), "Nonaxisymmetric Turbine End Wall Design - Part II: Experimental Validation," ASME J. Turbomach., 122, pp. 286-293.

Harvey, N. W. (2008), "Some Effects of Non-Axisymmetric End Wall Profiling on Axial Flow Compressor Aerodynamics. Part I: Linear Cascade Investigation," ASME paper No. GT-2008-50990, Berlin, Germany.

Harvey, N. W. and Offord, T. P. (2008), "Some Effects of Non-Axisymmetric End Wall Profiling on Axial Flow Compressor Aerodynamics. Part II: Multi-Stage HPC CFD Study," ASME paper No. GT-2008-50991, Berlin, Germany.

Harvey, N. W., Rose, M. G., Shahpar, S., Taylor, M. D., Hartland, J. and Gregory-Smith, D. G. (2000), "Nonaxisymmetric Turbine End Wall Design - Part I: Three-Dimensional Linear Design System," ASME J. Turbomach., 122, pp. 278-285.

Hatman, A. and Wang, T. (1999), "A Prediction Model for Separated-Flow Transition," ASME J. Turbomach., 121, pp. 594-602. 
Hildebrand, T. and Fottner, L. (1999), "A Numerical Study of the Influence of Grid Refinement and Turbulence Modeling on the Flow Field Inside a Highly Loaded Turbine Cascade," ASME J. Turbomach., 121, pp. 709-716.

Hjärne, J., Chernoray, V., Larsson, J. and Löfdahl, L. (2007), "Numerical Validations of Secondary Flows and Loss Development Downstream of a Highly Loaded Low Pressure Turbine Outlet Guide Vane Cascade," ASME paper No. GT-2007-27712, Montreal, Canada.

Hodson, H. P. and Dominy, R. G. (1987a), "The Off-Design Performance of a Low-Pressure Turbine Cascade," ASME J. Turbomach., 109, pp. 201-209.

Hodson, H. P. and Dominy, R. G. (1987b), "Three-Dimensional Flow in a Low-Pressure Turbine Cascade at Its Design Condition," ASME J. Turbomach., 109, pp. 177-185.

Hoheisel, H., Kiock, R., Lichtfuss, H. J. and Fottner, L. (1987), "Influence of Free-Stream Turbulence and Blade Pressure Gradient on Boundary Layer and Loss Behaviour of Turbine Cascades," ASME J. Turbomach., 109, pp. 210-219.

Holley, B. M. (2004), "Surface Measurements of Flow in a Plane Turbine Cascade," Ph.D. thesis, University of Connecticut, Storrs, CT, USA.

Hourmouziadis, J. (1989), "Aerodynamic Design of Low Pressure Turbines," Blading Designs for Axial Turbomachines, number 8 in 'AGARD-LS-167', Advisory Group for Aeronautical Research and Development, pp. 1-40.

Houtermans, R., Coton, T. and Arts, T. (2004), "Aerodynamic Performance of a Very High Lift Low Pressure Turbine Blade With Emphasis on Separation Prediction," ASME J. Turbomach., 126, pp. 406-413. 
Ingram, G., Gregory-Smith, D. and N.Harvey (2005), "Investigation of a Novel Secondary Flow Feature in a Turbine Cascade With End Wall Profiling," ASME J. Turbomach., 127, pp. 209-214.

Ingram, G. L., Gregory-Smith, D. G., Rose, M. G., Harvey, N. W. and Brennan, G. (2002), "The Effect of End-wall Profiling on Secondary Flows and Loss Development in a Turbine Cascade," ASME paper No. GT-2002-30339, Amsterdam, The Netherlands.

Jones, W. P. and Launder, B. E. (1972), "The Prediction of Laminarization with a TwoEquation Model of Turbulence," International Journal of Heat and Mass Transfer, 15, pp. 301-314.

Kacker, S. C. and Okapuu, U. (1982), "A Mean Line Prediction Method for Axial Flow Turbines Efficiency," Journal of Engineering for Power, 104, pp. 111-119.

Kopper, F. C., Milanot, R. and Vancot, M. (1981), "Experimental Investigation of Endwall Profiling in a Turbine Vane Cascade," AIAA Journal, 19, pp. 1033-1040.

Langston, L. S. (2001), "Secondary Flows in Axial Turbines - A Review," Annals of the New York Academy of Sciences, 934, pp. 11-26.

Langston, L. S., Nice, M. L. and Hooper, R. M. (1976), "Three-Dimensional Flow Within a Turbine Cascade Passage," ASME paper No. 76-GT-50, New Orleans, USA.

Langtry, R. B., Menter, F. R., Likki, S. R., Suzen, Y. B. and Huang, P. G. (2004), "A Correlation-Based Transition Model Using Local Variables Part II - Test Cases and Industrial Applications," ASME paper No. GT2004-53454, Vienna, Austria.

Launder, B. E. and Sharma, B. I. (1974), "Application of the Energy Dissipation Model of Turbulence to the Calculation of Flow Near a Spinning Disc," Letters in Heat and Mass Transfer, 1, pp. 131-138. 
Lewis, W. E. (1965), "Fixed-Direction Probes for Aerodynamic Measurements," Proceedings of the Institution of Mechanical Engineers, 1965-1966, pp. 141-151.

Mahallati, A. (2003), "Aerodynamics of a Low-Pressure Turbine Airfoil Under Steady and Periodically Unsteady Conditions," Ph.D. thesis, Carleton University, Ottawa, Canada.

Mahallati, A., McAuliffe, B. R., Sjolander, S. A. and Praisner, T. J. (2007), "Aerodynamics of a Low-Pressure Turbine Airfoil at Low-Reynolds Numbers Part 1 - Steady Flow Measurements," ASME paper No. GT-2007-27347, Montreal, Quebec, Canada.

Marchal, P. (1980), "Etude des ecoulements secondaires en grille daubes de detente," Ph.D. thesis, Universit Libre de Bruxelles, Brussels, Belgium.

Marchal, P. and Sieverding, C. H. (1977), "Secondary Flows within Turbomachinery Bladings," Secondary Flows in Turbomachines, number 11 in 'AGARD-CP-214', Advisory Group for Aeronautical Research and Development, pp. 1-19.

Mayle, R. E. (1991), "The Role of Laminar-Turbulent Transition in Gas Turbine Engines," ASME J. Turbomach., 113, pp. 560-569.

McAuliffe, B. (2003), "An Experimental Study of Flow Control Using Blowing for a LowPressure Turbine Airfoil," Master's thesis, Carleton University, Ottawa, Canada.

McAuliffe, B. R. and Yaras, M. I. (2005), "Separation-Bubble-Transition Measurements on a Low-re Airfoil Using Particle Image Velocimetry," ASME paper No. GT-2005-68663, Reno-Tahoe, Nevada, USA.

Menter, F. R., Langtry, R. B., Likki, S. R., Suzen, Y. B. and Huang, P. G. (2004), "A Correlation-Based Transition Model Using Local Variables Part I - Model Formulation," ASME paper No. GT2004-53452, Vienna, Austria. 
Menter, R. F. (1994), "Two-Equation Eddy-Viscosity Turbulence Models for Engineering Applications," AIAA Journal, 32, pp. 1598-1605.

Moffat, R. J. (1982), "Contributions to the Theory of Single-Sample Uncertainty Analysis," ASME J. Fluids Eng., 104, pp. 250-258.

Moore, J. and Adhye, R. Y. (1985), "Secondary Flows and Losses Downstream of a Turbine Cascade," ASME J. Turbomach., 107, pp. 961-968.

Naylor, E. M. J., Dueas, C. O., Miller, R. J. and Hodson, H. P. (2008), "Optimisation of Non-Axisymmetric Endwalls in Compressor S-Shaped Ducts," ASME paper No. GT-2008-50448, Berlin, Germany.

O'Donnell, F. K. and Davies, M. R. D. (2000), "Turbine Blade Entropy Generation Rate Part 2: The Measured Loss," ASME paper No. GT-2000-266, Munich, Germany.

Patterson, D. J. and Hoeger, M. (1986), "The Effect of Reynolds Number and Velocity Distribution on LP Turbine Cascade Performance," ASME paper No. 86-GT-271, Dussledorf, Germany.

Perdichizzi, A. and Dossena, V. (1993), "Incidence Angle and Pitch-Chord Effects on Secondary Flows Downstream of a Turbine Cascade," ASME J. Turbomach., 115, pp. 383-391.

Piggush, J. and Simon, T. (2005), "Flow Measurements in a First Stage Nozzle Cascade Having Endwall Contouring, Leakage and Assembly Features," ASME paper No. GT2005-68340, Reno-Tahoe, Nevada, USA.

Popović, I. (2005), "Measured Steady and Unsteady Aerodynamic Performance of a Family of Three Highly-Loaded Low-Pressure Turbine Cascades," Master's thesis, Carleton University, Ottawa, Canada. 
Popovic, I., Zhu, J., Dai, W., Sjolander, S. A., Praisner, T. J. and Grover, E. A. (2006), "Aerodynamics of a Family of Three Highly Loaded Low-Pressure Turbine Airfoils: Measured Effects of Reynolds Number and Turbulence Intensity in Steady Flow," ASME paper No. GT-2006-91271, Barcelona, Spain.

Praisner, T. J., Allen-Bradely, E., Grover, E. A., Knezevici, D. C. and Sjolander, S. A. (2007), "Application of Non-Axisymmetric Endwall Contouring to Conventional and High-Lift Turbine Airfoils," ASME paper No. GT-2007-27579, Montreal, Quebec, Canada.

Praisner, T. J. and Clark, J. P. (2007a), "Predicting Transition in Turbomachinery-Part I: A Review and New Model Development," ASME J. Turbomach., 129, pp. 1-13.

Praisner, T. J. and Clark, J. P. (2007b), "Predicting Transition in Turbomachinery-Part II: Model Validation and Benchmarking," ASME J. Turbomach., 129, pp. 14-22.

Praisner, T. J., Grover, E. A., Knezevici, D. C., Popovic, I. and Sjolander, S. A. (2008), "Toward the Expansion of Low-Pressure-Turbine Airfoil Design Space," ASME paper No. GT-2008-50898, Berlin, Germany.

Praisner, T. J. and Smith, C. R. (2006), "The Dynamics of the Horseshoe Vortex and Associated Endwall Heat Transfer Part II: Time-Mean Results," ASME J. Turbomach., 128, pp. 755-762.

Prümper, H. (1972), "Application of Boundary Layer Fences in Turbomachinery," Number II-3 in 'AGARD-CP-496', Advisory Group for Aeronautical Research and Development, pp. 315-331.

Roberts, S. K. and Yaras, M. I. (2003), "Measurements and Prediction of Free-Stream Turbulence and Pressure-Gradient Effects on Attached-Flow Boundary-Layer Transition," ASME paper No. GT-2003-38261, Atlanta, Georgia, USA. 
Rodger, P. (1992), "Establishing Two-Dimensional Flow in a Large Scale Planar TurbineCascade," Master's thesis, Carleton University, Ottawa, Canada.

Rose, M. G. (1994), "Non-Axisymmetric Endwall Profiling in the HP NGV's of an Axial Flow Gas Turbine," ASME paper No. 94-GT-249, Hague, Netherlands.

Rose, M. G., Harvey, N. W., Seaman, P., Newman, D. A. and McManus, D. (2001), "Improving the Efficiency of the Trent $500 \mathrm{HP}$ Turbine Using Non-Axisymmetric End Walls: Part II Experimental Validation," ASME paper No. 2001-GT-0505, 37th Aerospace Sciences Meeting and Exhibit, New Orleans, Louisiana, USA.

Sauer, H., Mller, R. and Vogeler, K. (2001), "Reduction of Secondary Flow Losses in Turbine Cascades by Leading Edge Modifications at the Endwall," ASME J. Turbomach., 123, pp. 207-213.

Sauer, H. and Wolf, H. (1994), "The Influence of the Inlet Boundary Layers on the Secondary Losses of Turbine Stages," Technology Requirements for Small Gas Tubines, number 28 in 'AGARD-CP-537', Advisory Group for Aeronautical Research and Development, p. 16.

Schlichting, H. and Gersten, K. (2000), Boundary Layer Theory, 8 ed., Springer, Berlin; New York.

Shahpar, S., Lapworth, B. L., Pablos, T. D. and Taylor, M. (1999), "A Linear Approach to the Multi-Parameter Design of Three-Dimensional Turbomachinery Blades," AIAA paper No. AIAA-1999-363, 37th Aerospace Sciences Meeting and Exhibit, Reno, NV, USA.

Sharma, O. P. and Butler, T. L. (1987), "Predictions of Endwall Losses and Secondary Flows in Axial Turbine Cascades," ASME J. Turbomach., 109, pp. 229-236. 
Sieverding, C. H. (1985), "Recent Progress in the Understanding of Basic Aspects of Secondary Flows in Turbine Blade Passages," Journal of Engineering for Gas Turbines and Power, 107, pp. 248-257.

Sieverding, C. H. and Van Den Bosche, P. (1983), "Use of Coloured Smoke to Visualize Secondary Flows in a Turbine Blade Cascade," J. Fluid Mech., 134, pp. 85-89.

Sjolander, S. A. (1975), "The Endwall Boundary Layer in an Annular Cascade of Turbine Nozzle Guide Vanes," Master's thesis, Carleton University, Ottawa, Canada.

Taylor, E. S. (1971), "Boundary Layers, Wakes and Losses in Turbomachines," Gas Turbine Laboratory Report No. 105, Massachusetts Institute of Technology.

Tremblay, B. (1990), "Measurement and Correlation of Off-Design Performance of AxialFlow Turbines," Master's thesis, Carleton University, Ottawa, Canada.

Vera, M., del la Rosa Blanco, E., Hodson, H. P. and Vazquez, R. (2009), "Endwall Boundary Layer Development in an Engine Representative Four-Stage Low Pressure Turbine Rig," ASME J. Turbomach., 131, pp. 011017.

Wang, H. P., Olson, S. J., Goldstein, R. J. and Eckert, E. (1997), "Flow Visualization in Linear Turbine Cascade of High Performance Turbine Blades," ASME J. Turbomach., 119, pp. 1-8.

Warner, R. and Tran, M. (1987), "Recent Developments to Improve High-Pressure and Intermediate-Pressure Turbine Efficiency," Proceedings of the Institution of Mechanical Engineers, number C278/87 in 'Turbomachinery: Effciency Prediction and Improvement: International Conference, pp. 287-292.

Weiss, A. P. and Fottner, L. (1995), "The Influence of Load Distribution on Secondary Flow in Straight Turbine Cascades," ASME J. Turbomach., 117, pp. 133-141.

White, F. M. (1974), Viscous Fluid Flow, McGraw-Hill Inc., New York. 
Wilcox, D. C. (1993), Turbulence Modeling for CFD, DCW Industries, Inc.

Wisler, D. (1998), "The Technical and Economic Relevance of Understanding Boundary Layer Transition in Gas Turbines," Workshop on Boundary Layer Transition in Turbomachines, number NASA/CP-1998-206958 in 'Minnowbrook II'.

Yamamoto, A. and Nouse, H. (1988), "Effects of Incidence on Three-Dimensional Flows in a Linear Turbine Cascade," ASME J. Turbomach., 110, pp. 486-496.

Yan, J., Gregory-Smith, D. G. and Walker, P. J. (1990), "Secondary Flow Reduction in a Nozzle Guide Vane by Non-Axisymmetric End-Wall Contouring," ASME paper No. 99-GT-339, Indianapolis, IN, USA.

Yaras, M. I. (1987), "Flow in the Tip Gap of a Rectilinear Turbine Cascade," Master's thesis, Carleton University, Ottawa, Canada.

Yaras, M. I. (1990), "Measurements and Prediction of Tip-Clearance Effects in a Linear Turbine Cascade," Ph.D. thesis, Carleton University, Ottawa, Canada.

Yaras, M. I. (2001), "Measurements of the Effects of Pressure-Gradients History of Separation-Bubble Transition," ASME paper No. 2001-GT-0193, New Orleans, LA, USA.

Yaras, M. I. and Sjolander, S. A. (1989), "Losses in the Tip-Leakage Flow of a Planar Cascade of Turbine Blades," Secondary Flows in Turbomachines, number 20 in 'AGARD-CP-496', Advisory Group for Aeronautical Research and Development, pp. $1-12$.

Zess, G. and Thole, K. (2002), "Computational Design and Experimental Evaluation of Using a Leading Edge Fillet on a Gas Turbine Vane," ASME J. Turbomach., 124, pp. $167-175$. 
Zhang, X. F. (2002), "Hot-Film Measurements of the Steady and Unsteady Boundary Layer Development on a Low-Pressure Turbine Airfoil," Master's thesis, Carleton University, Ottawa, Canada.

Zhang, X. F. and Hodson, H. (2005), "Combined Effects of Surface Trips and Unsteady Wakes on the Boundary Layer Development of an Ultra-High-Lift LP Turbine Blade," ASME J. Turbomach., 121, pp. 479-488.

Zorić, T. (2006), "Comparative Study of Secondary Losses for 3 Highly-Loaded LowPressure Turbine Cascades," Master's thesis, Carleton University, Ottawa, Canada.

Zorić, T., Popović, I., Sjolander, S. A., Praisner, T. and Grover, E. (2007a), "Comparative Investigation of Three Highly Loaded LP Turbine Airfoils: Part I - Measured Profile and Secondary Losses at Design Incidence," ASME paper No. GT-2007-27537, Montreal, Quebec, Canada.

Zorić, T., Popović, I., Sjolander, S. A., Praisner, T. and Grover, E. (2007b), "Comparative Investigation of Three Highly Loaded LP Turbine Airfoils: Part II - Measured Profile and Secondary Losses at Off-Design Incidence," ASME paper No. GT-2007-27538, Montreal, Quebec, Canada.

Zweifel, O. (1945), "The spacing of turbo-machine blading, especially with large angular deflection," Brown Boveri Review, 32, pp. 436-444. 


\section{Appendix A}

\section{Calibration Coefficients for}

\section{Pressure Transducers}

This appendix outlines the method used to determine the calibration coefficients for the pressure transducers. As discussed in Section 3.6.2, the transducer output voltage $(E)$ varies linearly with applied pressure $(\Delta P)$. With the transducers plumbed in parallel, the water micromanometer described by Benner (2003) was used to apply pressures spanning their operating range. Sample data for the DRAL501DN and DRAL505DN calibrations are presented in Figures A.1 and A.2, respectively.

Linear regression analysis was used to determine the slope $\left(C_{1}\right)$ and zero pressure voltage offset $\left(C_{2}\right)$. During the data reduction procedure, the slope is assumed to be that of the most recent calibration and the zero voltage offset is corrected by wind off data points taken prior to and after a test. Typical values for $C_{1}$ and $C_{2}$, and the correlation coefficient $\left(R^{2}\right)$ showing the goodness of fit are shown in Tables A.1 and A.2 for the DRAL501DN and DRAL505DN transducers. 


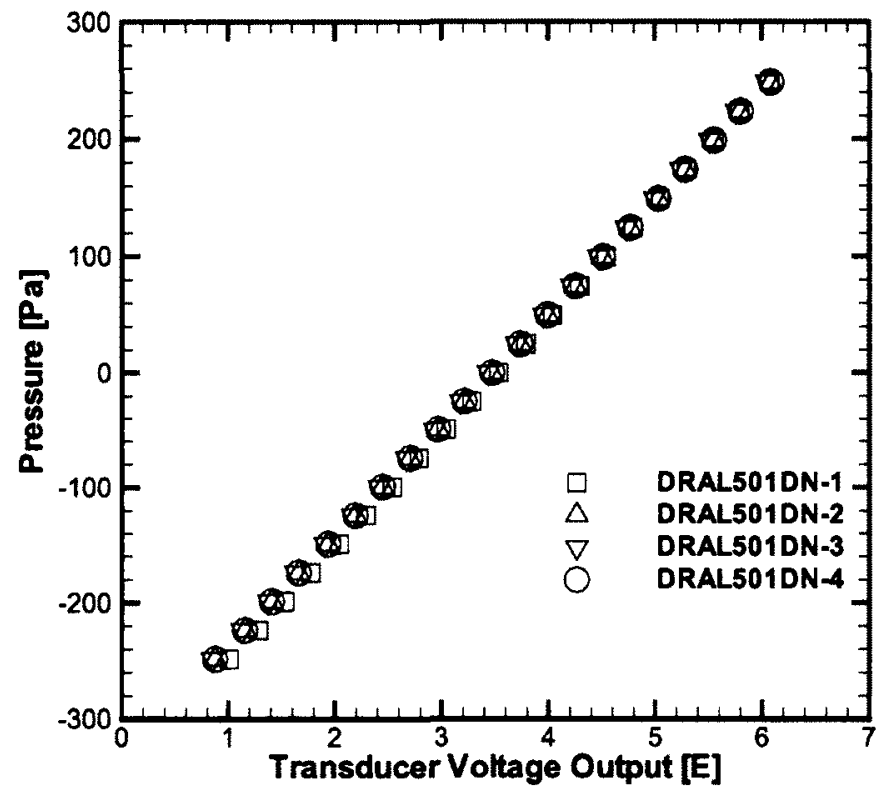

Figure A.1: Sample calibration curves for DRAL501DN pressure transducers

Table A.1: DRAL501DN calibration coefficients

\begin{tabular}{lccc}
\hline \hline & $C_{1}$ & $C_{2}$ & $R^{2}$ \\
\hline DRAL501DN-1 & 98.98188 & -350.05318 & 0.99997 \\
DRAL501DN-2 & 96.17397 & -334.24026 & 0.99998 \\
DRAL501DN-3 & 96.36172 & -331.62704 & 0.99997 \\
DRAL501DN-4 & 96.10707 & -334.14643 & 0.99997 \\
\hline \hline
\end{tabular}

Table A.2: DRAL505DN calibration coefficients

\begin{tabular}{lccc}
\hline \hline & $C_{1}$ & $C_{2}$ & $R^{2}$ \\
\hline DRAL505DN-1 & 492.236389 & -1702.188354 & 1.00000 \\
DRAL505DN-2 & 496.095734 & -1740.421875 & 1.00000 \\
DRAL505DN-3 & 487.285614 & -1701.984009 & 1.00000 \\
DRAL505DN-4 & 493.507263 & -1727.341553 & 0.99988 \\
DRAL505DN-5 & 493.452026 & -1710.529175 & 1.00000 \\
DRAL505DN-6 & 490.101837 & -1721.021606 & 1.00000 \\
DRAL505DN-7 & 491.169525 & -1716.926392 & 0.99996 \\
DRAL505DN-8 & 490.024109 & -1712.026978 & 0.99996 \\
\hline \hline
\end{tabular}




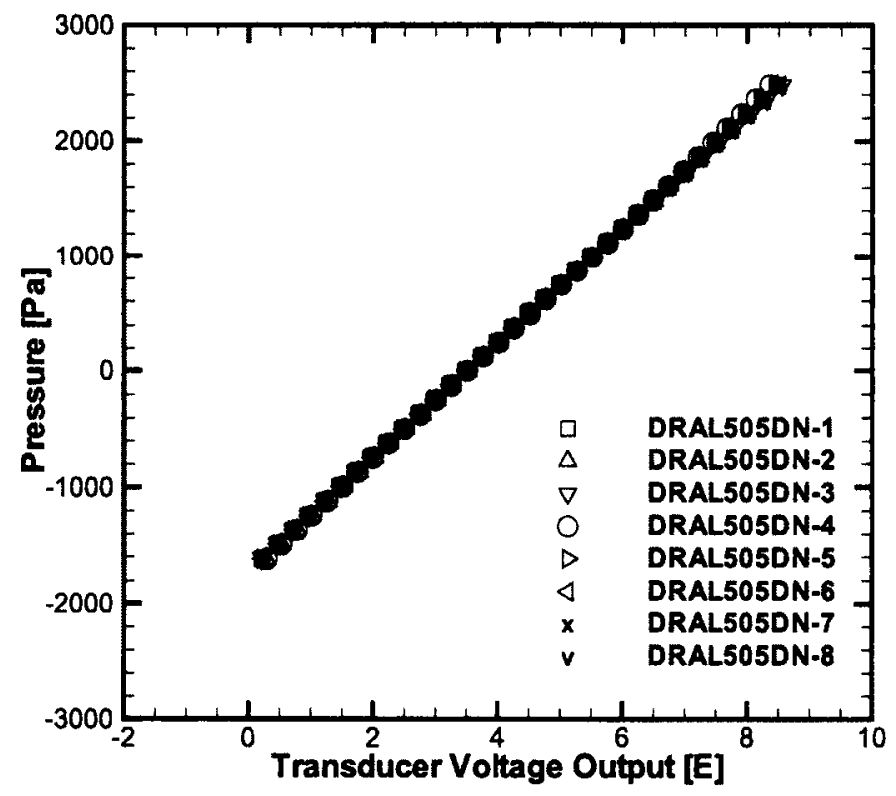

Figure A.2: Sample calibration curves for DRAL505DN pressure transducers 


\section{Appendix B}

\section{Calibration and Data Reduction Procedures for Three-Hole Pneumatic Probe}

This appendix summarizes the calibration and data reduction procedures for the threehole pressure probe. The probe was introduced in Section 3.9 .2 where it was shown schematically in Figure 3.6. The jet of the calibration apparatus was documented by Mahallati (2003) at several axial locations over the operating range using a Pitot-static probe and the results are presented in Figure 3.25 of his thesis. To calibrate the jet, the probe was first aligned geometrically along the axis of the nozzle, then pitched and yawed $\pm 3^{\circ}$ in $0.25^{\circ}$ increments. The total and static pressures were recorded from the orientation where the $P_{o}$ was highest and $P$ was lowest. These pressures were normalized by the reference dynamic pressure to form the jet calibration coefficients for total pressure $\left(C_{P o}\right)$, static pressure $\left(C_{P s}\right)$ and dynamic pressure $\left(C_{q}\right)$ as such: 


$$
\begin{gathered}
C_{P o}=\frac{P_{o}-P_{C 1}}{P_{C 1}-P_{C 2}} \\
C_{P s}=\frac{P-P_{C 2}}{P_{C 1}-P_{C 2}} \\
C_{q}=\frac{P_{o}-P}{P_{C 1}-P_{C 2}}=1+C_{P_{o}}-C_{P s}
\end{gathered}
$$

The probe calibrations for the present work were conducted with the probe tip located $x=32 \mathrm{~mm}$ downstream of the nozzle. At this location the results from Figure 3.25 (Mahallati, 2003) were confirmed using the procedure outlined above. The three-hole probe was first aerodynamically aligned in the jet, then calibrated over a range of $\phi= \pm 20^{\circ}$ in increments of $0.5^{\circ}$. At each measurement orientation, the pressure measured at each port of the probe $\left(P_{h}\right)$ was corrected for the nozzle calibration at the measurement plane as follows:

$$
C_{P h}=\frac{\frac{P_{h}-P_{C 2}}{P_{C 1}-P_{C 2}}-C_{P s}}{C_{q}} \quad \text { for } h=1,2,3
$$

The method of Lewis (1965) is used to determine following six non-dimensional calibration coefficients from the three port pressures:

$$
\begin{gathered}
K_{1}=\frac{P_{1}-P}{P_{o}-P}=C_{P 1} \\
K_{12}=\frac{P_{1}-P_{2}}{P_{o}-P}=C_{P 1}-C_{P 2} \\
K_{13}=\frac{P_{1}-P_{3}}{P_{o}-P}=C_{P 1}-C_{P 3} \\
K_{23}=\frac{P_{2}-P_{3}}{P_{o}-P}=C_{P 2}-C_{P 3} \\
K_{\phi 2}=\frac{P_{1}-P_{2}}{P_{1}-P_{3}}=\frac{K_{12}}{K_{13}}
\end{gathered}
$$




$$
K_{\phi 3}=\frac{P_{1}-P_{3}}{P_{1}-P_{1}}=\frac{1}{K_{\phi 2}}
$$

Sample calibration curves are shown in Figure B.1. The probe was calibrated for the cascade inlet and outlet nominal velocities $(25 \mathrm{~m} / \mathrm{s}$ and $40 \mathrm{~m} / \mathrm{s})$. The corresponding probe Reynolds numbers based on tip width (3350 and 5350) are considered to be in the high range, and only $K_{12}, K_{13}$ and $K_{23}$ show a slight sensitivity. Further details regarding three-hole probe sensitivity to Reynolds number can be found in Appendix B of McAuliffe (2003).
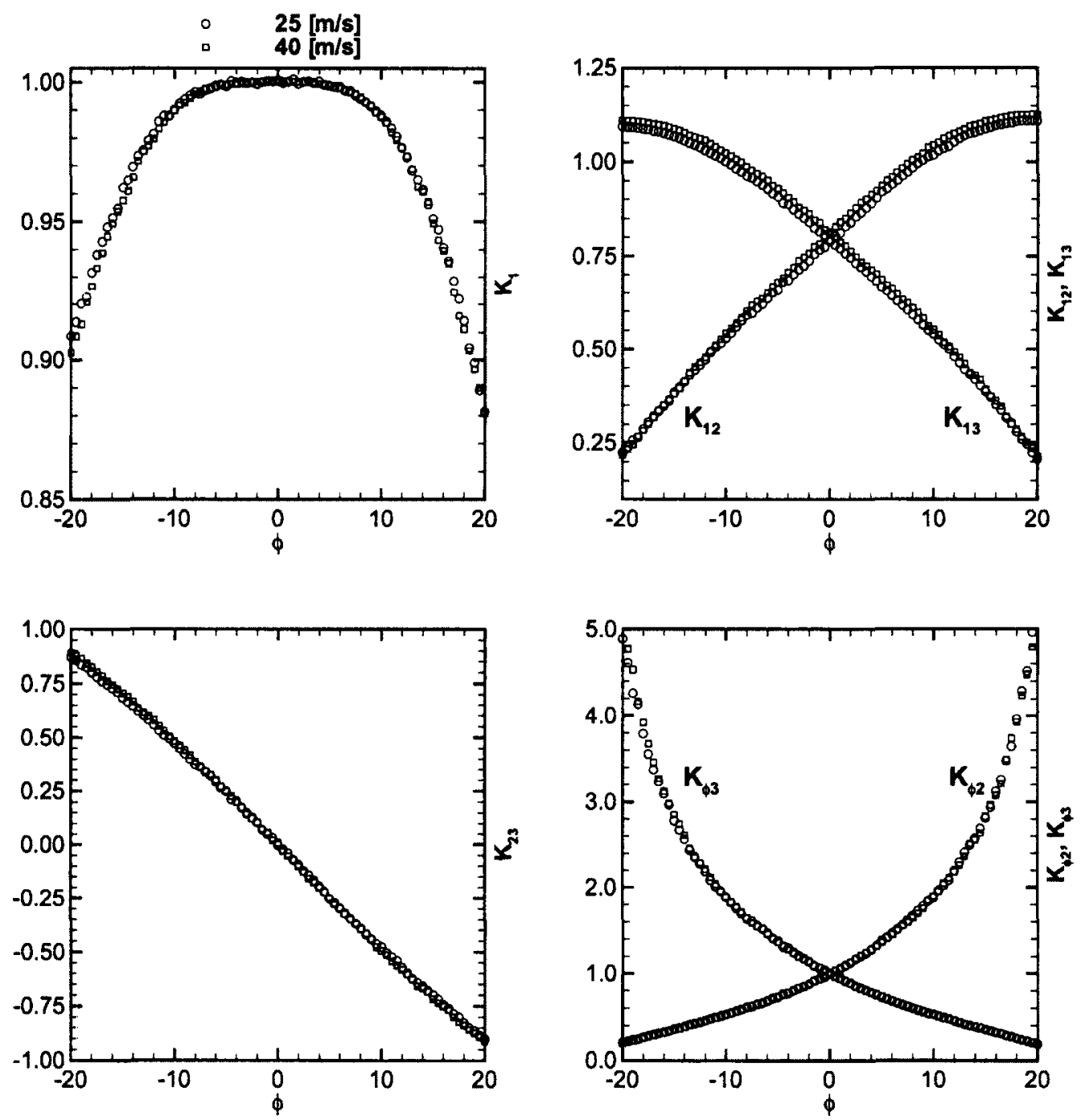

Figure B.1: Three-hole probe curves 
Once the pitch characteristics of the probe have been determined in a known flow, the probe can be used to documented an unknown two-dimensional velocity field. In the unknown flow, the port pressures $P_{1}, P_{2}$ and $P_{3}$ are measured and it is determined if $P_{2}$ or $P_{3}$ is greater. Linear interpolation is then used on the calibration curves as follows:

1. If $P_{2}$ reads high, $K_{\phi 2}$ is used to determine $\phi$. Otherwise $\phi$ is found from $K_{\phi 3}$

2. If $P_{2}$ reads high, $\phi$ used to determine $q=P_{o}-P$ from $K_{12}$. Otherwise $q$ is found from $K_{13}$

3. With $\phi$ and $q$ known, the static pressure $P$ is found from $K_{1}$

The probe is used in non-nulling mode. The angle of the probe axis relative to the axial direction of the cascade $(\varphi)$ is set using a digital protractor to $\pm 0.1^{\circ}$. The flow angle relative to the cascade is then found by:

$$
\alpha=\varphi+\phi
$$

In order to reduce measurement error originating from large flow misalignments, the probe was aligned with the expected flow vector at the inlet and outlet of the cascade. 


\section{Appendix $\mathrm{C}$}

\section{Calibration and Data Reduction}

\section{Procedures for Seven-Hole}

\section{Pneumatic Probe}

This appendix outlines the calibration and data reduction procedures for the seven-hole pressure probe. The probe was introduced in Section 3.9.3 where it was shown schematically in Figure 3.7. The calibration procedure used is that of Gallington (1980), as referred to by Gerner and Maurer (1980). It is appropriate for flow misalignments of $\pm 70^{\circ}$ in pitch and yaw for documenting incompressible flow. The procedure is divided into two regimes, the low angle low-angle regime where the central port (7) reads high, or the high angle regime where one of the surrounding ports (1-6) senses the maximum pressure. The division of angular space for the head of the probe is shown in Figure C.1, reproduced from Gerner et al. (1984).

As with the three-hole probe, the seven port pressures are corrected for the jet calibration (Equation B.4) prior to generating the calibration coefficients. 


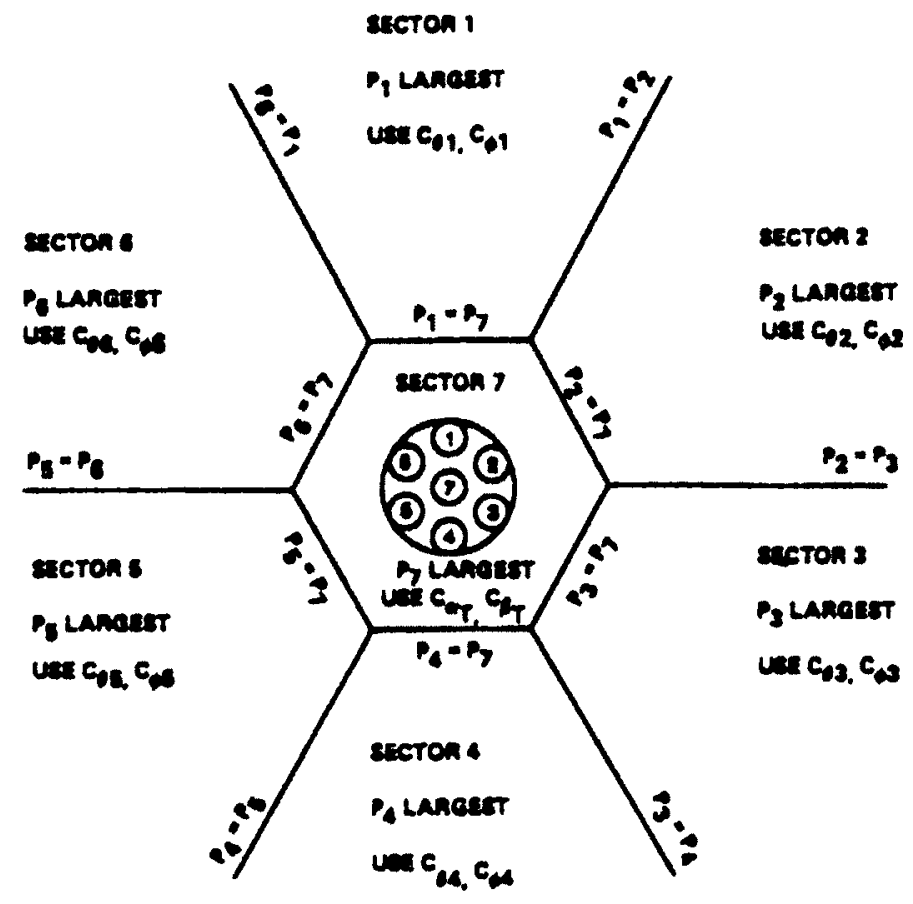

Figure C.1: Division of angular space (reproduced from Gerner et al. (1984)

\section{C.1 Low-Angle Regime}

For low flow misalignments, typically on the order of $20^{\circ}$ the central port 7 senses the highest pressure. In the low angle regime, all seven port pressures are used to define the following non-dimensional coefficients:

$$
\begin{aligned}
& C_{1}=\frac{P_{4}-P_{1}}{P_{7}-\bar{P}_{1-6}} \\
& C_{2}=\frac{P_{3}-P_{6}}{P_{7}-\bar{P}_{1-6}} \\
& C_{3}=\frac{P_{2}-P_{5}}{P_{7}-\bar{P}_{1-6}}
\end{aligned}
$$

Where $\bar{P}_{1-6}$ is the average pressure sensed at ports one through six. The numerator of each of the coefficients is formed using pressures from opposing faces of the probe. So each 
coefficient is most sensitive to angular misalignments on one of three planes separated by $60^{\circ} . P_{7}$ approximates the local total pressure sensed by the probe thus the denominator is a pseudo dynamic pressure.

The coefficients defined in Equations C.1-C.3 can be used to define two angular coefficients for the tangential coordinate system shown in Figure C.2.

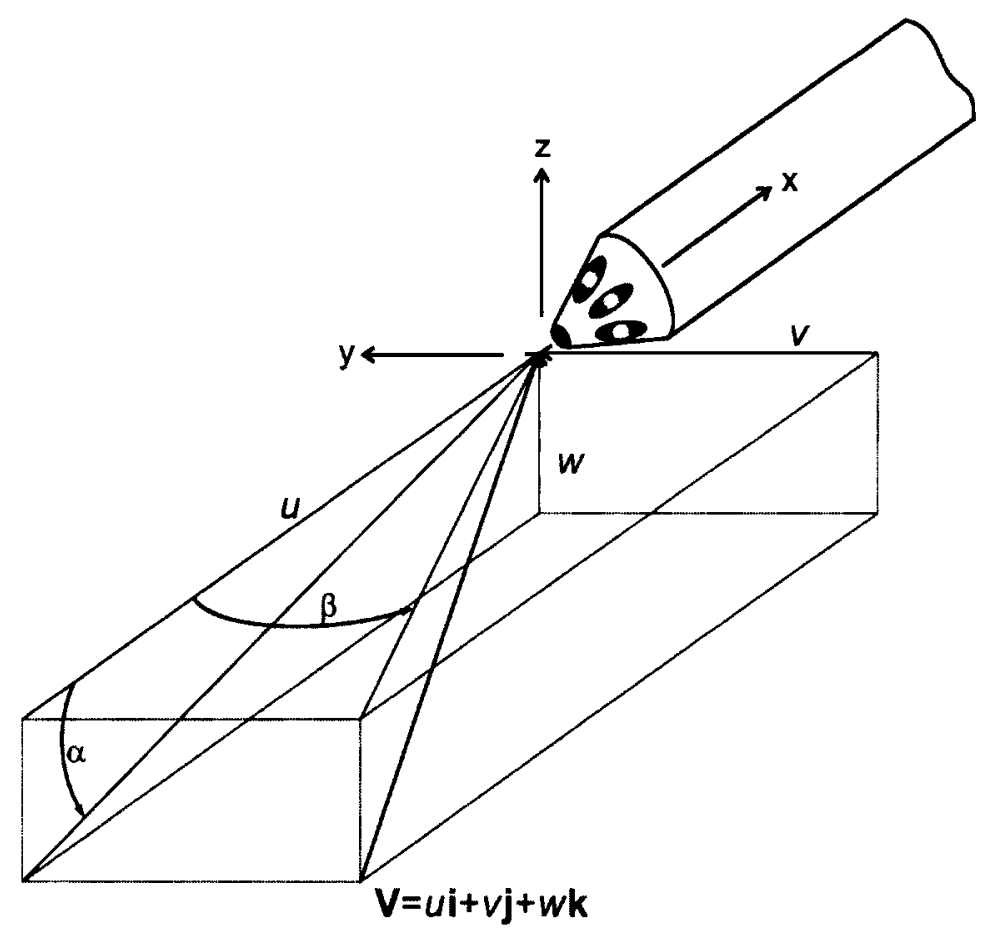

Figure C.2: Tangential coordinate system for low-angle regime (reproduced from Benner, 2003)

$$
\begin{gathered}
C_{\alpha}=\frac{2 \cdot C_{1}+C_{2}-C_{3}}{3} \\
C_{\beta}=\frac{C_{2}+C_{3}}{\sqrt{3}}
\end{gathered}
$$

The coordinate system in Figure C.2 is oriented such that $\alpha$ is positive when hole 4 reads the highest pressure, and $\beta$ is positive when holes holes 2,3 read the highest pressure. With 
the probe calibrated in a known flow, the local pitch angle $(\alpha)$, spanwise angle $(\beta)$, total pressure coefficient $\left(C_{o}\right)$ and dynamic pressure coefficient $\left(C_{q}\right)$ can be related to Equations C.4 and C.5 as follows:

$$
\begin{gathered}
\alpha=f_{1}\left(C_{\alpha}, C_{\beta}\right) \\
\beta=f_{2}\left(C_{\alpha}, C_{\beta}\right) \\
C_{o}=f_{3}\left(C_{\alpha}, C_{\beta}\right) \\
C_{q}=f_{4}\left(C_{\alpha}, C_{\beta}\right)
\end{gathered}
$$

with the total and dynamic-pressure coefficients defined as:

$$
\begin{gathered}
C_{o}=\frac{P_{7}-P_{o}}{P_{7}-\bar{P}_{1-6}} \\
C_{q}=\frac{P_{7}-\bar{P}_{1-6}}{P_{o}-P}
\end{gathered}
$$

Figures C.3, C.4, C.5 and C.6 show the calibration data for sector 7. Each of the figures show contour plots demonstrating the smoothness of the distribution of the coefficient in question. The data reduction procedure for the low-angle regime uses linear interpolation in a lookup table represented by the contour plots. The figures also contain two other plots showing cut planes through the angular space at $\alpha=0$ and $\beta=0$. 


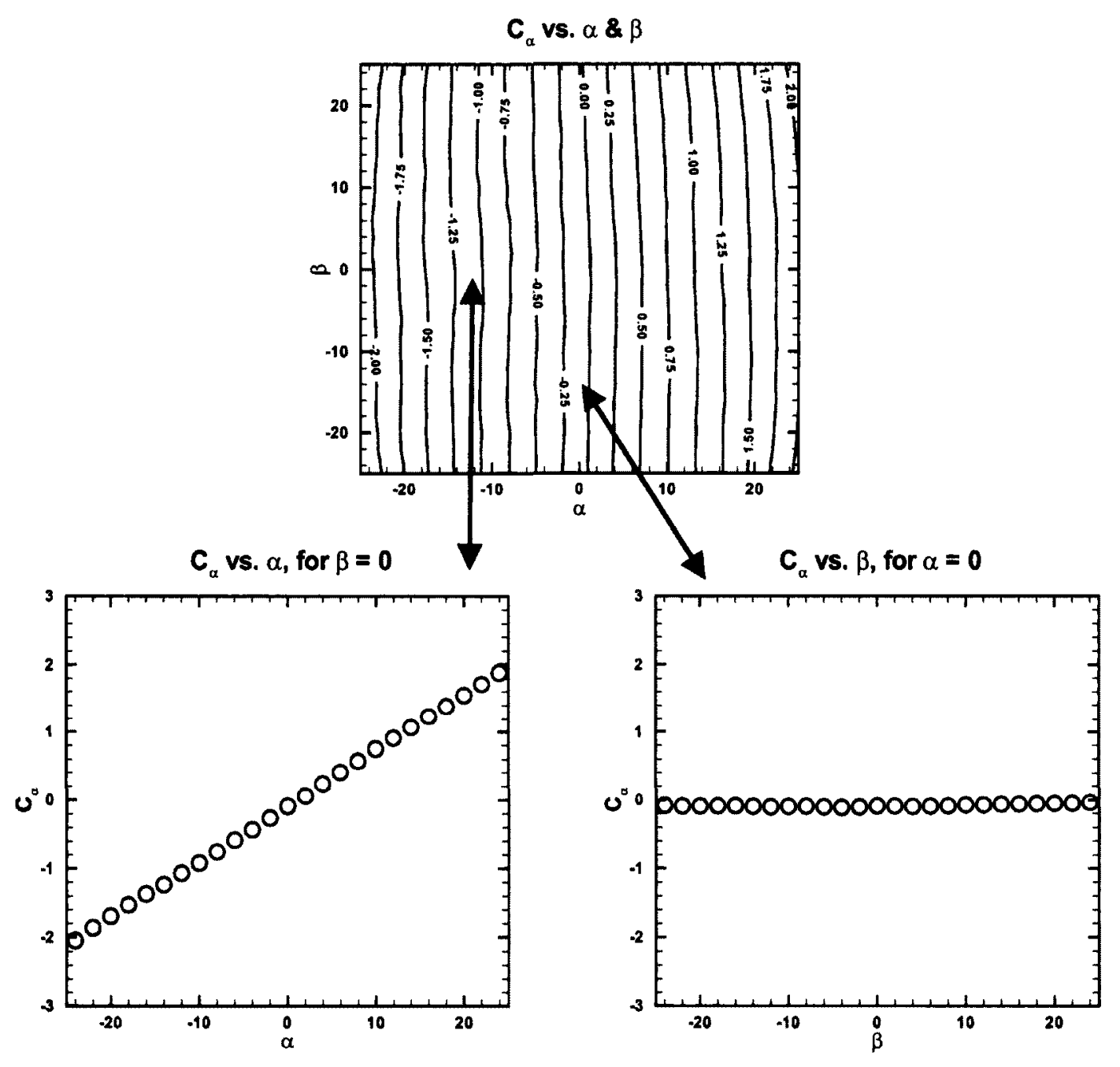

Figure C.3: $C_{\alpha}$ calibration data for sector 7 


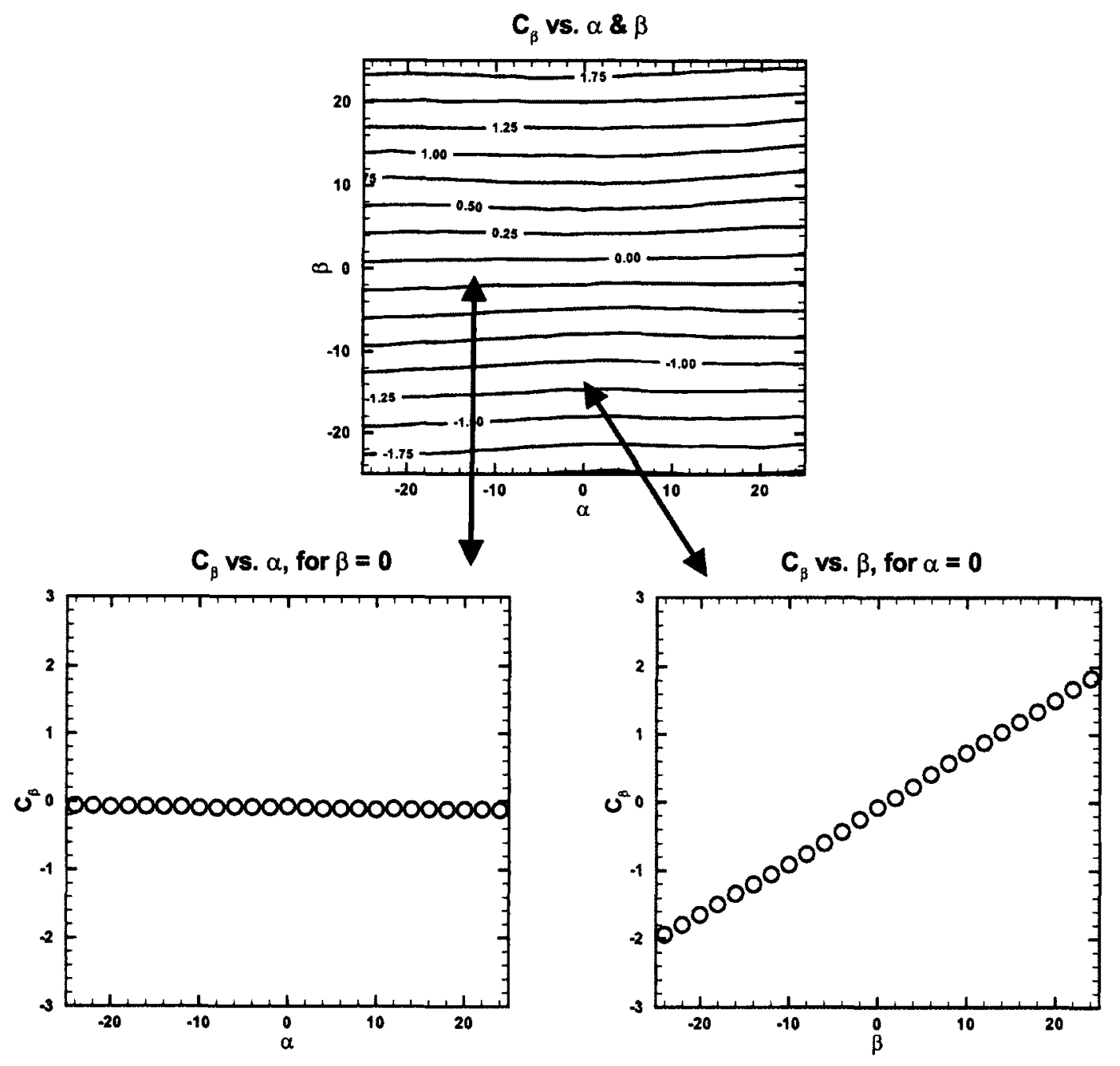

Figure C.4: $C_{\beta}$ calibration data for sector 7 


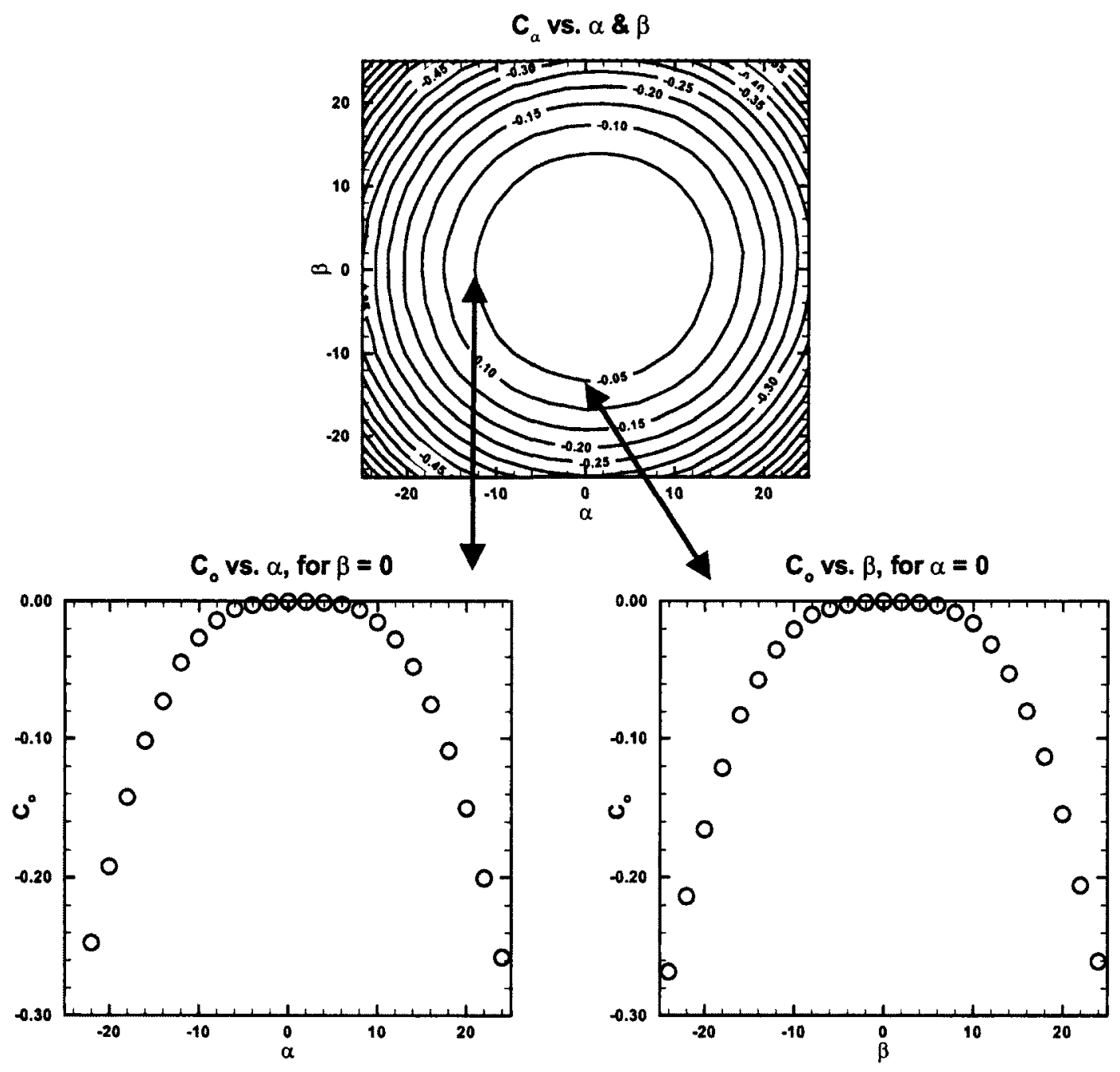

Figure C.5: $C_{o}$ calibration data for sector 7 


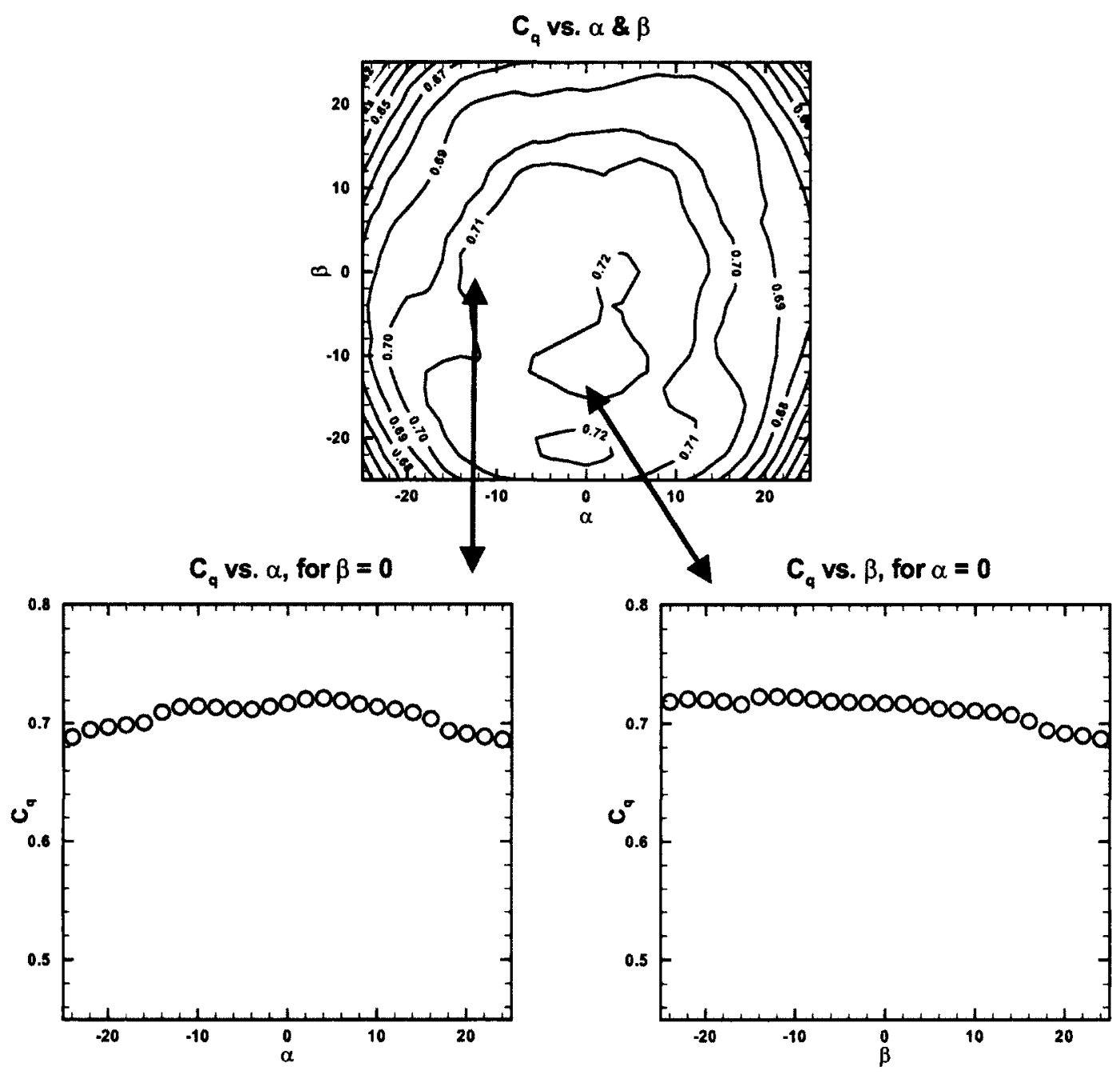

Figure C.6: $C_{q}$ calibration data for sector 7 


\section{C.2 High-Angle Regime}

When the head of the probe is exposed to high angular misalignment, the flow will separate from the leeward face of the probe. As such, it is necessary to define the calibration coefficients based solely using ports on the windward side. The high angle regime is used when one of the six peripheral ports reads the highest pressure. When this is the case, the coefficients are derived using the high reading port, it's two peripheral neighbors and $P_{7}$ (see Figure C.1).

Gallington (1980) recommends the use of a polar coordinate system as shown in Figure C.7 for defining the high-angle regime angular calibration coefficients as such:

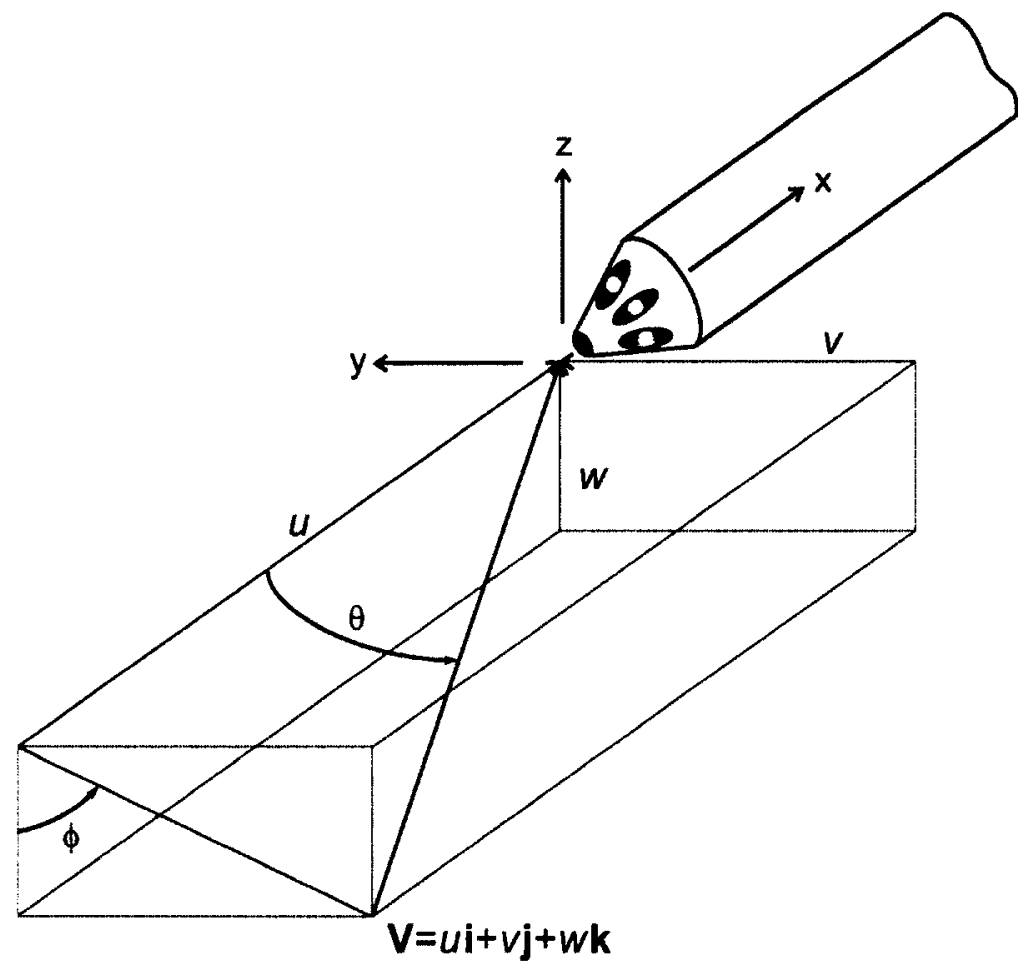

Figure C.7: Cylindrical coordinate system for high-angle regime (reproduced from Benner, 2003) 


$$
\begin{gathered}
C_{\theta_{n}}=\frac{P_{n}-P_{7}}{P_{n}-\left(\frac{P_{n-}+P_{n+}}{2}\right)} \\
C_{\phi_{n}}=\frac{P_{n-}-P_{n+}}{P_{n}-\left(\frac{P_{n-}+P_{n+}}{2}\right)}
\end{gathered}
$$

The subscript $n$ refers to the peripheral port reading the highest pressure. $n-$ and $n+$ designate the adjacent counterclockwise and clockwise peripheral ports to the high reading port (see Figure C.1). Equations C.12 and C.13 are the angular sensitivity coefficients and can be related to the pitch angle $(\theta)$, roll angle $(\phi)$, total pressure coefficient $\left(C_{o}\right)$ and dynamic pressure coefficient $\left(C_{q}\right)$ as follows:

$$
\begin{gathered}
\theta=f_{1}\left(C_{\theta_{n}}, C_{\phi_{n}}\right) \\
\phi=f_{2}\left(C_{\theta_{n}}, C_{\phi_{n}}\right) \\
C_{o}=f_{3}\left(C_{\theta_{n}}, C_{\phi_{n}}\right) \\
C_{q}=f_{4}\left(C_{\theta_{n}}, C_{\phi_{n}}\right)
\end{gathered}
$$

with the total and dynamic-pressure coefficients defined as:

$$
\begin{aligned}
C_{o}= & \frac{P_{n}-P_{o}}{P_{n}-\left(\frac{P_{n-}+P_{n+}}{2}\right)} \\
C_{q}= & \frac{P_{n}-\left(\frac{P_{n-}+P_{n+}}{2}\right)}{P_{o}-P}
\end{aligned}
$$

As with the low-angle regime, the probe is calibrated in a known flow to determine the relationships in Equations C.14 - C.17. The sample calibration data for sector 4 is shown 
in Figures C.8 - C.11. Each of the figures contain a contour plot showing the distribution of the coefficients. The data reduction procedure uses linear interpolation in a lookup table represented by these contour plots to determine the relationships in Equations C.14 - C.17. The figures also present cut planes through the data at $\phi=0$ and $\theta=40$ to help visualize the trends.

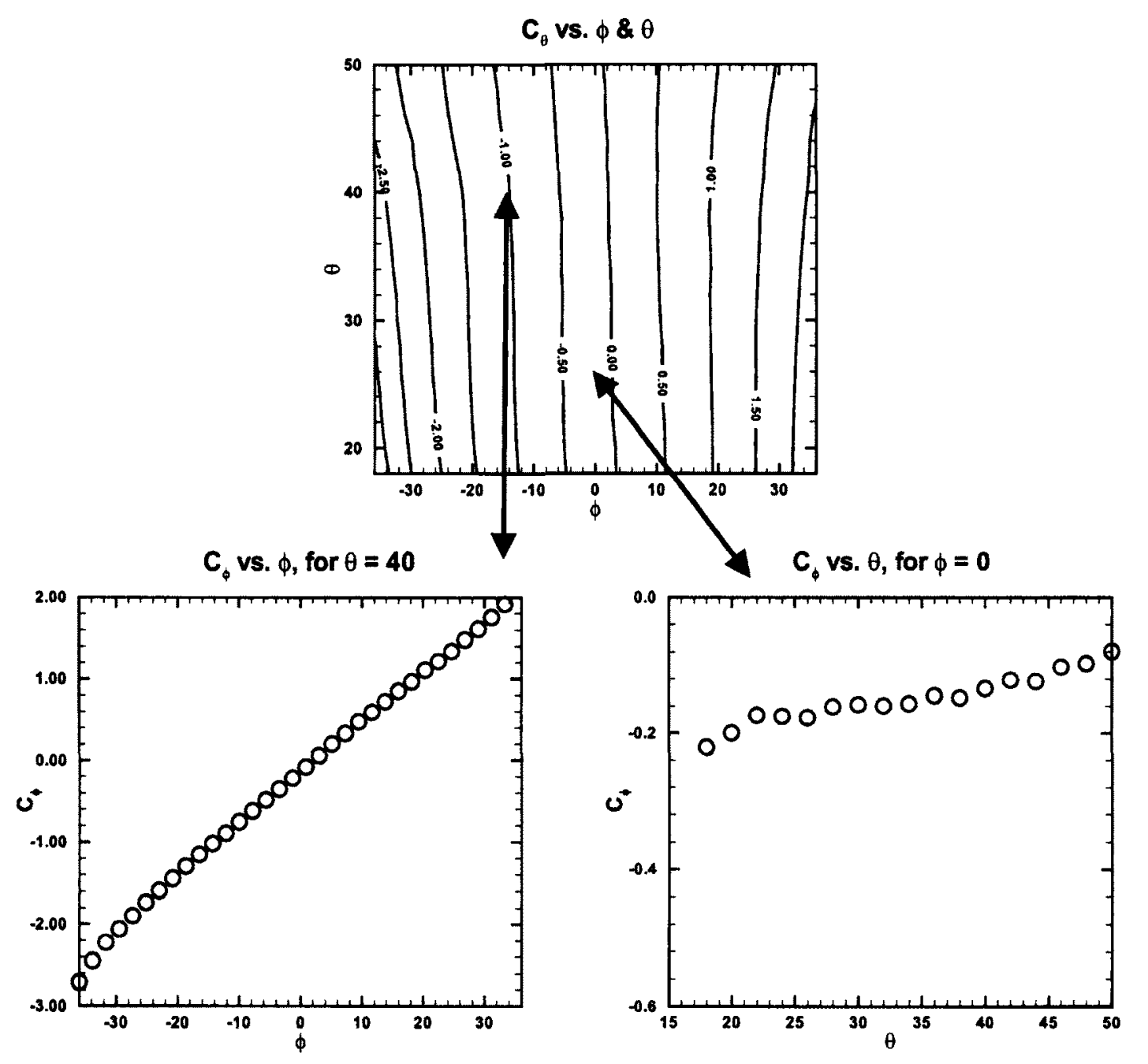

Figure C.8: $C_{\phi}$ calibration data for sector 4 


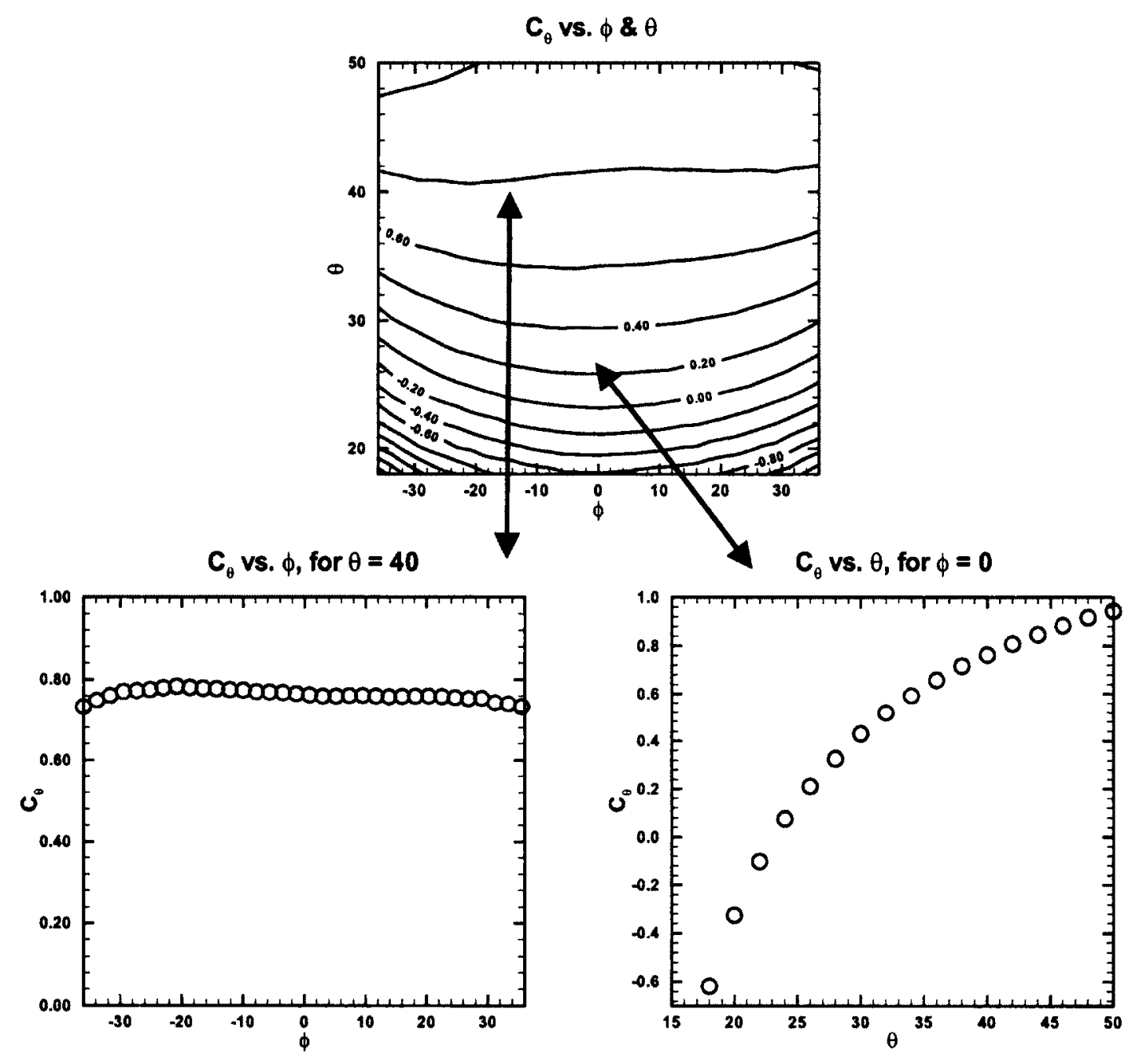

Figure C.9: $C_{\theta}$ calibration data for sector 4 


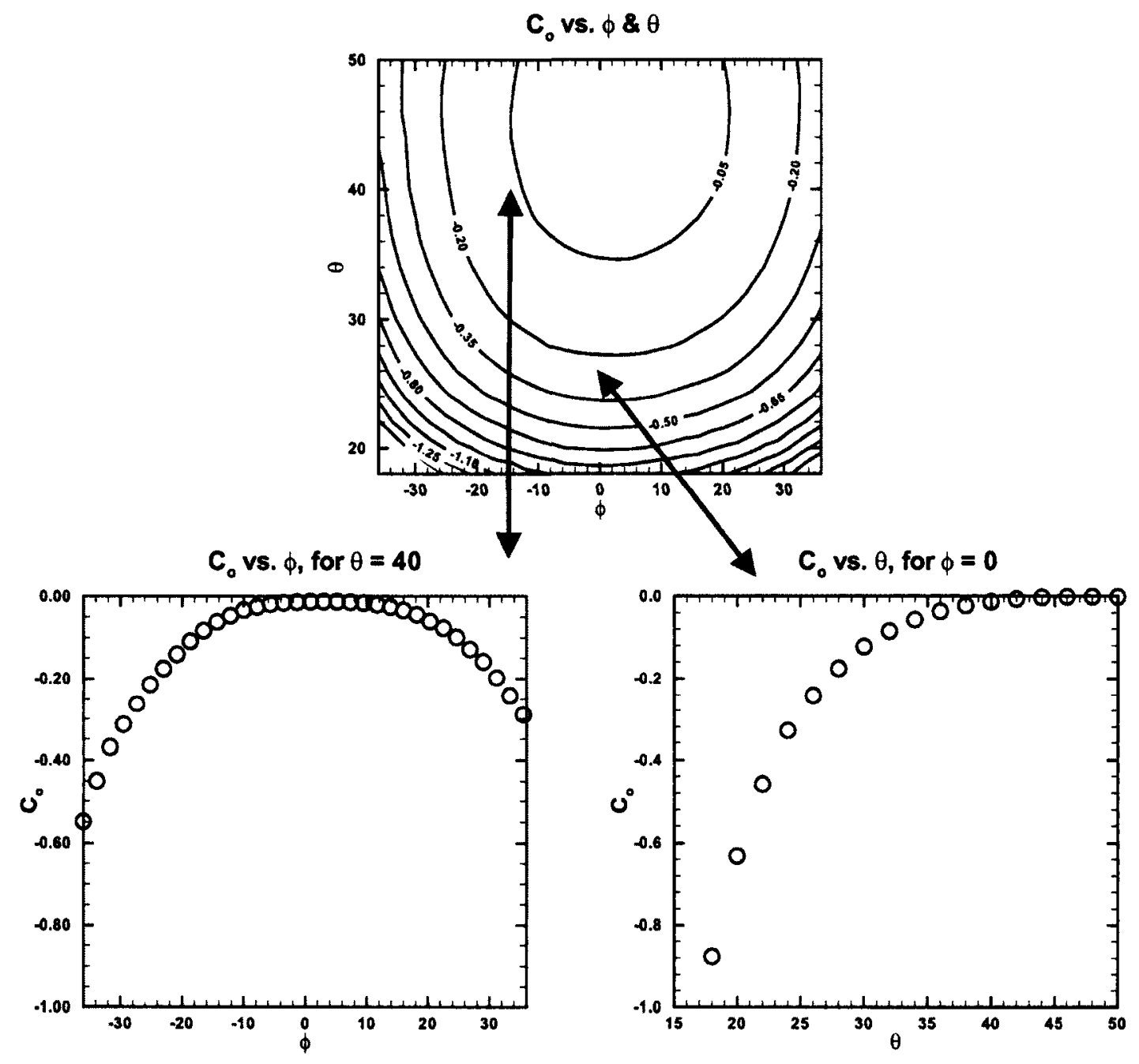

Figure C.10: $C_{0}$ calibration data for sector 4 


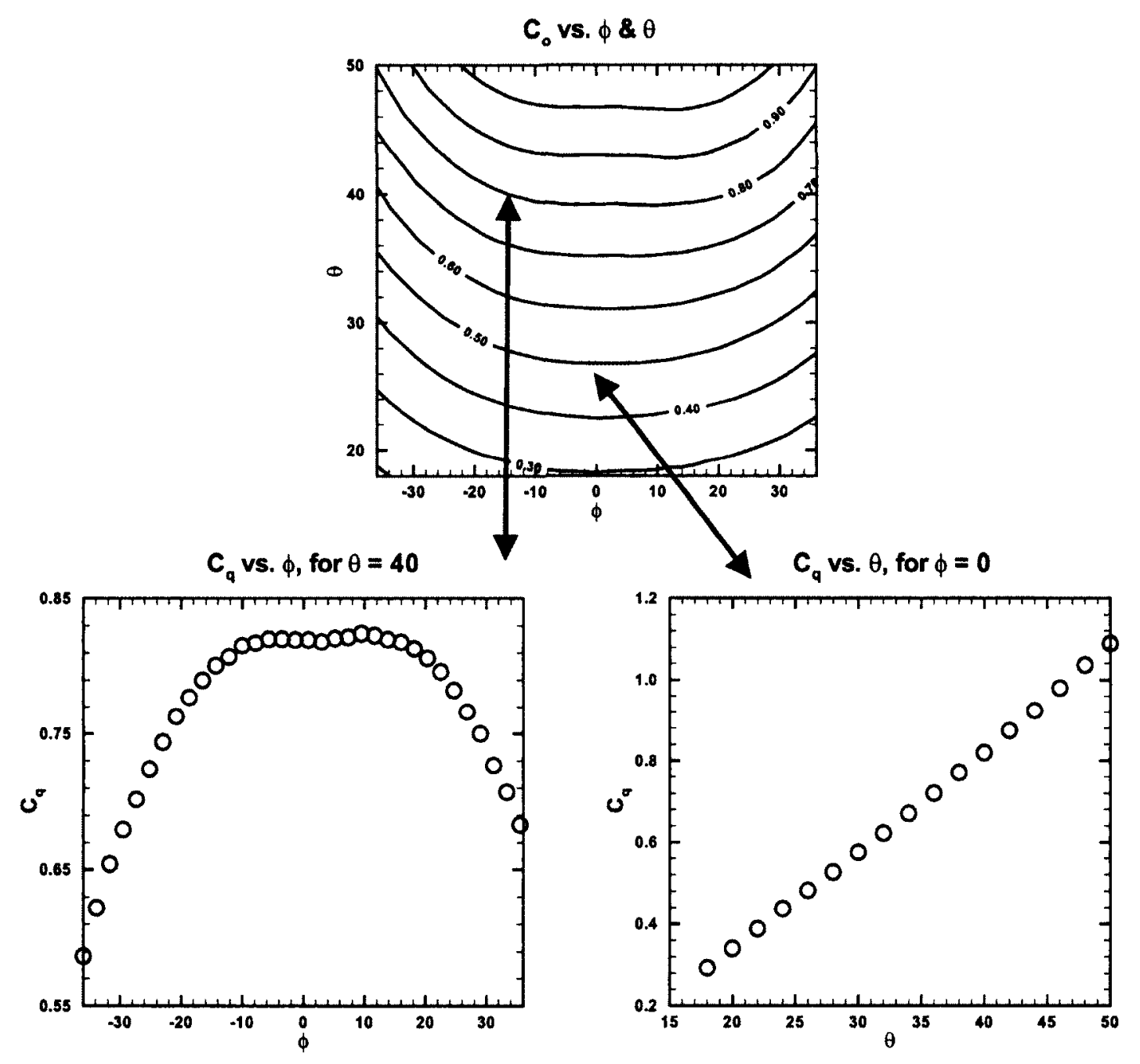

Figure C.11: $C_{q}$ calibration data for sector 4 